\title{
FoRMUlaÇÃo do MÉTOdo dOS ElEMENTOS DE CONTORNO PARA ANÁLISE DE CHAPAS COM ENRIJECEDORES
}

\section{Wilson Wesley Wutzow}

Dissertação apresentada à Escola de Engenharia de São

Calos da Universidade de São Paulo, como parte dos requisitos necessários para obtenção do título de Mestre em Engenharia de Estruturas.

Orientador: Prof. Assoc. João Batista de Paiva 
Ao Senhor Deus Soberano, à Késia aos meus pais - Wilson e Miriâ, aos meus irmãos - Mirian e Carlos 


\section{AgRAdeCimentos}

Ao Senhor nosso Deus, criador e mantenedor da vida.

Ao Prof. Associado Dr. João Batista de Paiva pela orientação dispensada nestes dois anos de pesquisa.

Aos meus pais, pelo empenho e motivação nos momentos difíceis.

À minha querida Késia, pela compreensão e apoio.

Ao CNPq pelo apoio financeiro.

Ao Prof. Titular Dr. Wilson Sérgio Venturini pela dedicação e paciência dispensada em tantas horas de aconselhamento fundamentais na criação deste trabalho, sobretudo pela grande amizade.

Á Universidade de São Paulo, em especial ao Departamento de Estruturas pela infraestrutura e facilidade proporcionadas na realização deste trabalho.

Aos amigos e Professores do Departamento de Engenharia Civil da Universidade Estadual de Maringá (UEM), em especial ao prof. Dr. Roberto Cruz Lessa pelos conselhos e amizade e também aos professores Peralta, Nara e Pigozo pelo apoio durante o desenvolvimento deste trabalho.

Aos amigos e Professores do SET, em especial aos amigos Leandro Vanalli, Rodrigo Ribeiro Paccola e Alberto Tomé pela amizade e companheirismo. 
SUMÁRIO

LiSTA DE FIGURAS vi vi v vis

LISTA DE GRÁFICOS ix

LISTA DE TABELAS - xi

LISTA DE SÍMBOLOS xii

Lista de Abreviaturas e Siglas $\quad$ xiii

RESUMO Xiv

$\begin{array}{lll}\text { ABSTRACT } & \text { xv }\end{array}$

1 INTRODUÇÃO 1

1.1 Revisão Bibliográfica $\quad 1$

1.2 Metodologia de trabalho 6

$\begin{array}{ll}1.3 \text { Objetivos deste trabalho } & 7\end{array}$

2 FUNDAMENTOS DA TEORIA DA ELASTICIDADE 9

2.1 Equações Básicas de teoria da elasticidade 9

3 SOLUÇÃO FUNDAMENTAL DE KELVIN E EQUAÇÃO INTEGRAL PARA O PROBLEMA ELÁSTICO BIDIMENSIONAL

3.1 Métodos $\quad 15$

3.2 Solução Fundamental de Kelvin $\quad 16$

3.3 Equação Integral de Contorno Para o Problema Elástico Plano 18

4 MÉTODO DOS ELEMENTOS DE CONTORNO 23

4.1 Equação Algébrica $\quad 23$

4.2 Integrais Analíticas 33 
4.3 Integração Numérica $\quad 38$

5 SISTEMA LINEAR SUAVE - MÍNIMOS QUADRADOS 45

5.1 Conceituação Básica Sobre o Método dos Mínimos Quadrados

5.2 Mínimos Quadrados por Sub-Região $\quad 47$

5.3 Mínimos Quadrados por Elemento 49

5.4 Exemplos de Aplicação $\quad 51$

5.5 Conclusões Parciais $\quad 62$

6 ENRIJECEDORES

6.1 Sub-Regiões $\quad 65$

6.2 Condensação das Incógnitas do Contorno do Enrijecedor $\quad 70$

6.3 Exemplos de Aplicação $\quad 74$

6.4 Conclusões Parciais $\quad 85$

7 INTERFACE GRÁFICA $\quad \mathbf{8 6}$

7.1 Pequena Revisão Bibliográfica $\quad 86$

7.2 Utilização da Interface Gráfica

7.3 Exemplos de aplicação $\quad 95$

7.4 Conclusões parciais $\quad 103$

8 CONCLUSÕES

$\begin{array}{ll}\text { ANEXO A - TEOREMA DE BETTI } & 106\end{array}$

$\begin{array}{ll}\text { ANEXO B - FunÇão Delta de Dirac } & 108\end{array}$

ANEXO C - FunÇÃo Delta de Kronecker 110

ANEXO D - INTEGRAIS ANALítiCAS NÃo SingULARES 112

$\begin{array}{ll}\text { ANEXO E - INTEgRais ANALÍtiCAS Singulares } & 127\end{array}$

REFERÊNCIAS BIBLIOGRÁFICAS

Bibliografia Complementar $\quad 136$

APÊNDICE A - NOTAÇÃo INDICIAL $\quad 1^{\mathrm{A}}$

APÊNDICE B - Problema de VAlor de Contorno 
FIGURA 01 - (a) Elemento infinitesimal, (b) Forças de superfície................................ 11

FIGURA 02 - Solução para singularidade em ponto fonte no contorno........................... 20

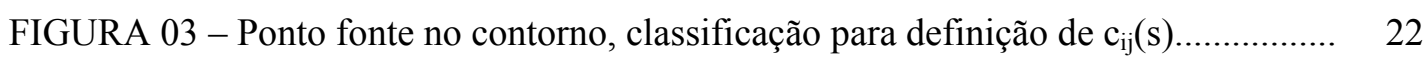

FIGURA 04 - Discretização do contorno em elementos................................................ 23

FIGURA 05 - Elemento linear isoparamétrico.............................................................. 24

FIGURA 06 - Posicionamento do ponto fonte.............................................................. 28

FIGURA 07 - Extrapolação da tensão interna ao elemento para os nós do contorno...... 33

FIGURA 08 - Casos para integração não singular........................................................ 35

FIGURA 09 - Transformação de coordenadas para integração analítica não singular com ponto fonte não alinhado com o elemento....................................... 35

FIGURA 10 - Funções de forma............................................................................. 36

FIGURA 11 - Casos para integração singular.......................................................... 38

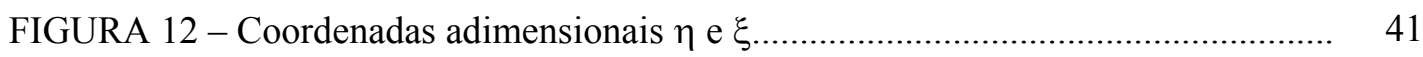

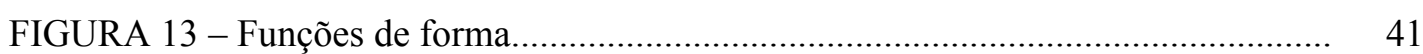

FIGURA 14 - Determinação dos ângulos para o sub-elemento....................................... 42

FIGURA 15 - Determinação dp comprimento para o sub-elemento.................................. 43

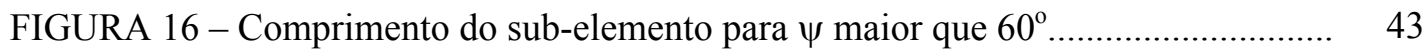

FIGURA 17 - Divisão do elemento em sub-elementos................................................... 44

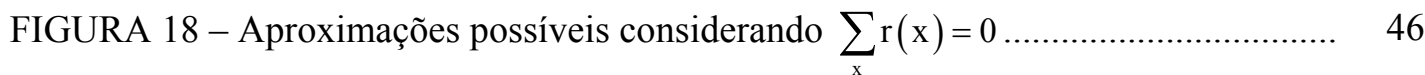

FIGURA 19 - Discretização do problema................................................................. 48

FIGURA 20 - Matrizes [H] e [G] originais que serão substituídas pelas novas matrizes

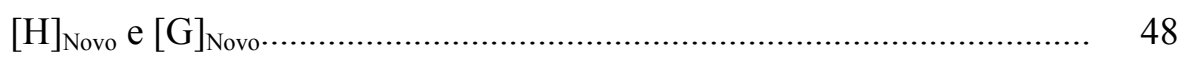

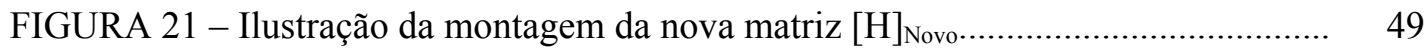




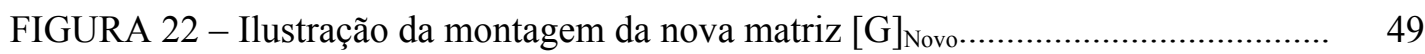

FIGURA 23 - Discretização do problema....................................................................... $\quad 50$

FIGURA 24 - Matrizes [H] e [G] originais que serão substituídas pelas novas matrizes

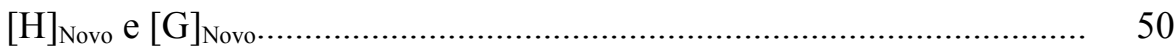

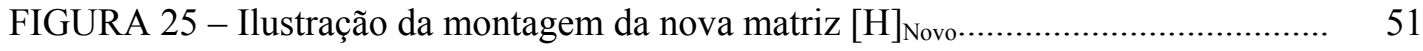

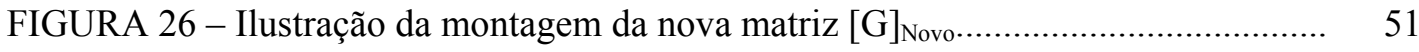

FIGURA 27 - Dados do exemplo.......................................................................... 52

FIGURA 28 - Malha 1 - 60 elementos........................................................................ 52

FIGURA 29 - Malha 2 - 600 elementos.................................................................... 52

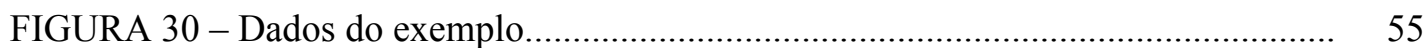

FIGURA 31 - Malha 1 - 80 elementos................................................................... 56

FIGURA 32 - Malha 1 - 800 elementos.................................................................... 56

FIGURA 33 - Dados do exemplo............................................................................. 58

FIGURA $34-1^{\text {a }}$. Malha, composta por 50 elementos................................................... 59

FIGURA $35-2^{\text {a }}$. Malha, composta por 500 elementos.................................................. 59

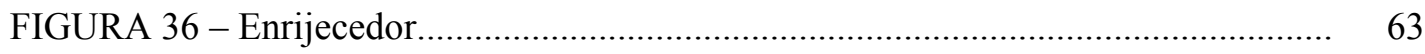

FIGURA 37 - Duas Sub-regiões............................................................................. 65

FIGURA 38 - Geometria e discretização do exemplo................................................... 68

FIGURA 39 - Sistema de equações do domínio 1......................................................... 68

FIGURA 40 - Sistema de equações do domínio 2.......................................................... 68

FIGURA 41 - Junção dos dois sistemas de equações num só............................................ $\quad 69$

FIGURA 42 - Aplicação das condições de equilíbrio....................................................... 69

FIGURA 43 - Rearranjo do sistema de equações............................................................. 70

FIGURA 44 - Enrijecedor com seus eixos locais $\overline{\mathrm{x}}$ e $\overline{\mathrm{y}}$.................................................. 70

FIGURA 45 - Nós centrais que recebem as contribuições da integração no contorno.... 71

FIGURA 46 - Transformações das incógnitas de deslocamento do contorno em incógnitas centrais....................................................................... 72

FIGURA 47 - Transformações das incógnitas de força do contorno em esforças resultantes do enrijecedor..................................................................... 73

FIGURA 48 - Dados do problema 1..................................................................... $\quad 75$

FIGURA 49 - Discretização do problema 1................................................................ $\quad 75$

FIGURA 50 - Dados físicos, geométricos e condições de contorno do exemplo 2......... 78

FIGURA 51 - Discretização empregada no exemplo 2................................................ 78

FIGURA 52 - Dimensões e condições de contorno simuladas no exemplo 3................. 80

FIGURA 53 - Discretização cujos resultados são apresentados no exemplo 3............... 80 
FIGURA 54 - Pascalina, a maquina de calcular desenvolvida pelo matemático, físico e filósofo francês Blasi Pascal..

FIGURA 55 - Geometria do problema sendo discretizada em arquivo *.dxf................. 89

FIGURA 56 - Tela principal do programa................................................................... $\quad 90$

FIGURA 57 - Inserção dos dados geométricos do problema........................................... $\quad 90$

FIGURA 58 - Dados gerais do problema.................................................................... 91

FIGURA 59 - configurações de cada uma das sub-regiões............................................. 91

FIGURA 60 - Configurações relacionadas a discretização do problema......................... 92

FIGURA 61 - Aplicação das condições de contorno...................................................... 93

FIGURA 62 - Facilidade de vizualização.................................................................... 93

FIGURA 63 - Núcleo de processamento: onde os cálculos são efetuados....................... 94

FIGURA 64 - Interface gráfica desenvolvida em Delphi com rotinas em OpenGl para visualização dos resultados..................................................................... 94

FIGURA 65 - Algumas configurações de visualização dos resultados............................ 95

FIGURA 66 - Dados do exemplo 1 do capítulo 5 ........................................................... 96

FIGURA 67 - Representação gráfica dos resultados do exemplo 1 do capítulo 5.......... 96

FIGURA 68 - Dados do exemplo 2 do capítulo 5 .......................................................... 97

FIGURA 69 - Representação gráfica dos resultados do exemplo 2 do capítulo 5.......... 97

FIGURA 70 - Dados do exemplo 3 do capítulo 5 .......................................................... 98

FIGURA 71 - Recursos de transparência dos mapas...................................................... 98

FIGURA 72 - Dados do problema 1 do capítulo 6 ............................................................ 99

FIGURA 73 - Representação gráfica dos resultados do exemplo 1 do capítulo 6........... 99

FIGURA 74 - Recursos que melhoram a visualização de concentração de tensões........ 100

FIGURA 75 - Dados do problema 2 do capítulo 6 .......................................................... 101

FIGURA 76 - Representação gráfica dos resultados do exemplo 2 do capítulo 6.......... 101

FIGURA 77 - Representação gráfica dos resultados do exemplo 3 do capítulo 6.......... 102

FIGURA 78 - Dados do problema 3 do capítulo 6..................................................... 103 


\section{LISTA DE GRÁFICOS}

GRÁFICO 1 - Deslocamentos da face inferior da viga................................................ 53

GRÁFICO 2 - Reação de apoio Fx do lado engastado................................................ 54

GRÁFICO 3 - Reação de apoio Fy do lado engastado.................................................. 54

GRÁFICO 4 - Deslocamentos da face inferior.......................................................... 56

GRÁFICO 5 -Reação de apoio Fx do lado totalmente engastado.................................. 57

GRÁFICO 6 -Reação de apoio Fy do lado totalmente engastado.................................. 57

GRÁFICO 7 - Deslocamentos em Y da face inferior da viga....................................... 60

GRÁFICO 8 - Reações de apoio em X na interface entre os domínio 1 e 2 da viga....... 60

GRÁFICO 9 - Reações de apoio em Y na interface entre os domínio 1 e 2 da viga...... 61

GRÁFICO 10 - Deslocamentos na direção X da interface entre os domínio 1 e 2 da

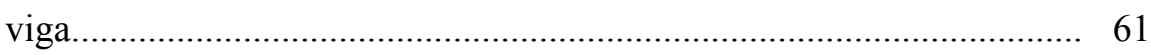

GRÁFICO 11 - Deslocamentos na direção Y da interface entre os domínio 1 e 2 da

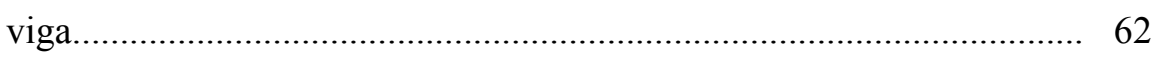

GRÁFICO 12 - Deslocamentos na direção X do enrijecedor........................................ 76

GRÁFICO 13 - Deslocamentos na direção Y do enrijecedor........................................... 76

GRÁFICO 14 - Esforços na direção X atuantes no enrijecedor....................................... 76

GRÁFICO 15 - Esforços na direção Y atuantes no enrijecedor....................................... 76

GRÁFICO 16 - Gráfico de $\partial \mathrm{u}_{\mathrm{x}} / \partial \mathrm{x}$ no enrijecedor obtido na condensação...................... 77

GRÁFICO 17 - Gráfico de $\partial \mathrm{u}_{\mathrm{y}} / \partial \mathrm{x}$ no enrijecedor obtido na condensação...................... 77

GRÁFICO 18 - Gráfico do momento fletor atuante no enrijecedor............................... 77

GRÁFICO 19 - Gráfico da variação da força normal entra as interfaces do

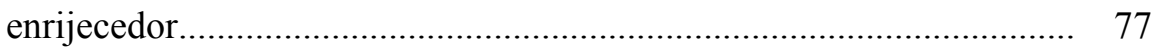

GRÁFICO 20 - Deslocamentos na direção X do enrijecedor........................................ 78

GRÁFICO 21 - Deslocamentos na direção Y do enrijecedor......................................... 78 
GRÁFICO 22 - Esforços na direção X atuantes no enrijecedor 79

GRÁFICO 23 - Esforços na direção Y atuantes no enrijecedor...................................... 79

GRÁFICO 24 - Gráfico de $\partial \mathrm{u}_{\mathrm{x}} / \partial \mathrm{x}$ no enrijecedor obtido na condensação...................... 79

GRÁFICO 25 - Gráfico de $\partial \mathrm{u}_{\mathrm{y}} / \partial \mathrm{x}$ no enrijecedor obtido na condensação...................... 79

GRÁFICO 26 - Gráfico do momento fletor atuante no enrijecedor................................ 79

GRÁFICO 27 - Gráfico da variação da força normal entra as interfaces do

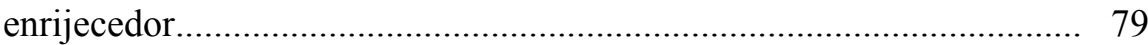

GRÁFICO 28 - Força cortante na duas faces do enrijecedor número 1 simulado com a técnica de sub-região....................................................................... 82

GRÁFICO 29 - Força cortante resultante que atua no enrijecedor número 1 simulado com a condensação de variáveis............................................................. 82

GRÁFICO 30 - Deslocamento no eixo $\mathrm{x}$ das duas faces do enrijecedor número 1 simulado com a técnica de sub-região

GRÁFICO 31 - Deslocamento médio no eixo x do enrijecedor número 1 simulado com a condensação de variáveis.

GRÁFICO 32 - Força cortante nas duas faces do enrijecedor número 1 simulado com a técnica de sub-região

GRÁFICO 33 - Força cortante resultante do enrijecedor número 1 simulado com a condensação de variáveis 
TABELA 01 - Deslocamentos em y da ponta do balanço................................................ 55

TABELA 02 - Deslocamentos em y da ponta do balanço............................................. 58

TABELA 03 - Deslocamentos em y da ponta do balanço............................................... 62

TABELA 04 - Deslocamentos em x no meio e na ponta do enrijecedor.......................... 77

TABELA 05 - Deslocamentos em y da ponto A......................................................... 80 


\section{LiSTA DE SÍMBOLOS}

$\alpha, \beta, \theta$ - Símbolos empregados na representação de ângulos ou constantes.

$\pi-\mathrm{Pi}=3.14159265358979 \ldots$

p - Forças de superfície.

b - Forças de volume.

$\sigma_{\mathrm{ij}}-$ Tensor de tensões.

$\varepsilon_{\mathrm{ij}}$ - Tensor de deformações.

$\mathrm{U}$ - Deslocamentos.

$\phi-$ Funções de forma.

$\eta$-Cossenos diretores.

E - Módulo de elasticidade.

$v$ - Coeficiente de Poisson.

$\mathrm{G}$ - Módulo de elasticidade transversal.

$\Omega$ - Domínio.

$\Gamma-$ Contorno.

$\Omega^{*}$ - Domínio do problema fundamental.

$\Gamma^{*}$ - Contorno do problema fundamental.

$\delta(\mathrm{s}, \mathrm{q})$ - Delta de Dirac.

$\delta_{\mathrm{ik}}-$ Delta de Kronecker.

$\mathrm{r}$ - Raio.

$\mathrm{s}$ - Ponto fonte.

$\mathrm{q}$ - Ponto campo. 


\section{Lista de Abreviaturas e Siglas}

MEC - Método dos elementos de Contorno

MEF - Método dos elemento finitos

EPT - Estado plano de tensão

EPD - Estado plano de deformação

CAD - Design assisted by computer ( projeto ou desenho realizado com a utilização de computadores e softwares específicos).

$\mathrm{DXF}$ - formato de arquivo comum entre os programas que desenvolvem geometrias.

OpenGL - Biblioteca de rotinas gráficas desenvolvida pela Silicon Graphics. 


\section{RESUMO}

WUTZOW, W.W. (2003). Formulação do método dos elementos de contorno para análise de chapas com enrijecedores. São Carlos. 140p. Dissertação (Mestrado) - Escola de Engenharia de São Carlos, Universidade de São Paulo.

Neste trabalho, a formulação linear do método dos elementos de contorno - MEC, para elasticidade bidimensional, é empregada para estudo de domínios enrijecidos sendo os enrijecedores abordados de duas formas, a primeira muito conhecida trata-se da técnica de sub-região ou acoplamento MEC/MEC e a segunda também pelo mesmo tipo de acoplamento, mas agora condensando-se as variáveis do contorno para a linha central do enrijecedor. Esta técnica juntamente com a integração completamente analítica dos termos da equação Somigliana proporcionam bons resultados eliminando perturbações em enrijecedores finos. Com o intuito de obter melhores resultados aplica-se ainda a técnica de suavização do contorno por mínimos quadrados. Aspectos gráficos são abordados na criação do pré e pós processador, sendo o pré-processador um interpretador de arquivos de formato dxf e o pós-processador destinado a representação gráfica dos resultados através de mapas e isolinhas de tensão, deformação e deslocamentos.

Palavras-chaves: Enrijecedores, Elementos de Contorno, Representação Gráfica. 


\section{ABSTRACT}

WUTZOW, W.W. (2003). Formulation of the baoundary elements method for analysis of stiffned plates. São Carlos. 140p. Dissertação (Mestrado) - Escola de Engenharia de São Carlos, Universidade de São Paulo.

In this work, the boundary element metho - BEM, for two-dimensional elasticity, is used to analyse reinforced domains. The rigidity stiffener contribution are taking into account by two different ways: the first one by the sub-region technique or BEM/BEM coupling, and the second one, also based on BEM/BEM coupling, but now considering the variables defined along the central line of the stiffer. Analytical expressions were found to perform the integrals along boundary and interface elements, providing very good results characterized by the elimination of some perturbations that might occur when using stiffener with small rigidity. In order to obtains better and smooth results the least square method was used. Pre- and post-processors were developed and implemented for visualization of the input data and the final results. The pre-processor was written as a dxf reader program, while the post-processor as a counter map stress, strain and displacement iso-lines.

Keywords: Stiffnes, Boundary Elements, Graphical Representation. 
Apesar da aparente precocidade do método dos elementos de contorno, apenas cerca de 20 a 30 anos de existência do método, não se pode esquecer seus precursores, pesquisadores que com o avanço de seus estudos em fundamentos como desenvolvimento de soluções integrais para os mais diversos problemas físicos, tornaram viável a criação do método dos elementos de contorno como conhecemos hoje. Claro que é inegável reconhecer que muito há para ser desenvolvido ainda para que o método englobe as mais diversas situações. Problemas como anisotropia, não linearidade, ações dinâmicas, soluções autoadaptativas, análises tridimensionais, etc. São situações que apesar dos avanços obtidos, ainda existem muitos aspectos a serem desenvolvidos.

\subsection{REVISÃo BiBLIOGRÁFICA}

Neste item será apresentado apenas um breve histórico do desenvolvimento do método dos elementos de contorno e de seus precursores.

Ao citarmos os precursores do método dos elementos de contorno, não podemos esquecer de trabalhos como de ABEL (1823) que foi quem primeiro deduziu uma equação integral para solucionar um problema físico, que se tratava do pêndulo isócrono. Já LIOUVILLE (1837), transformou um problema de valor inicial em uma equação integral, e resolveu-a usando aproximações sucessivas. VOLTERRA (1884) obteve avanços estudando a distribuição de cargas elétricas na superfície de uma esfera utilizando equações integrais.

Já o primeiro estudo rigoroso das equações integrais deve-se a FREDHOLM (1903), que apresentou um estudo das aplicações das equações integrais lineares à solução de problemas de valor de contorno em elastostática, que posteriormente os pesquisadores 
soviéticos MUSKHELISHVILI (1953), MIKHLIN (1957), (1965) E KUPRADZE (1965) aplicaram para solução de problemas de elasticidade.

MASSONET (1965) descreve uma solução numérica para problemas de valores de tensões no contorno, formulado em termos de uma equação integral singular, vetorial de $2^{\underline{a}}$ espécie, baseada na solução de KELVIN. Já SYMM (1963) e JASWON (1963) introduziram um equacionamento do problema elástico bidimensional empregando variáveis reais e ainda mantendo uma função de tensão auxiliar sendo por isso chamado de método semidireto, usado na resolução de problema de potencial. Até então todas as formulações eram ditas métodos indiretos, já que a solução do problema era sempre obtida em termos de fontes fictícias aplicadas ao contorno, e que após a sua determinação, permitiam o cálculo das variáveis físicas do problema.

Foi RIZZO (1967) quem primeiro publicou um trabalho dando uma roupagem numérica para as equações integrais em problemas de elasticidade. Ele propôs uma formulação direta para as equações integrais. CRUZE (1969), (1973) foi o primeiro a estender esta formulação para o caso tridimensional. Posteriormente CRUZE \& VAMBUREN (1971) aplicam não-linearidade ao sólido tridimensional considerando a influência de uma fratura. LACHAT (1975), deu grande contribuição ao método introduzindo representações paramétricas dos elementos de superfície e das funções de aproximação das forças de superfície e deslocamentos, calculando as integrais numericamente pela quadratura de Gauss. A técnica de sub-regiões é empregada não só para modular corpos não homogêneos, mas como um recurso para facilitar a resolução do sistema final de equações, que passa assim a ser definido por blocos.

Inicialmente, método dos elementos de contorno foi conhecido como método das equações integrais de contorno, pois os problemas eram resolvidos através de equações integrais sobre o contorno do domínio. Posteriormente, BREBBIA (1978a e 1978b) tratou o método das equações integrais de contorno de uma maneira mais conveniente, chamando-o de método dos elementos de contorno. A formulação do método dos elementos de contorno foi elaborada, primeiramente, a partir de aproximações das equações integrais obtidas através de algum princípio clássico, por exemplo, o teorema de BETTI (1872). Depois se verificou a possibilidade de se utilizar o método dos resíduos ponderados para obter a formulação do método dos elementos de contorno, tornando-a mais genérica e facilitando a combinação com outros métodos numéricos conforme BREBBIA et al. (1984). Pode-se observar na evolução do método dos elementos de contorno, os chamados métodos indiretos onde as 
variáveis envolvidas não são as variáveis físicas do problema e, os métodos diretos nos quais a formulação é desenvolvida considerando-se as variáveis reais do problema.

Conforme TELLES \& BREBBIA (1979, 1980a e 1980b), é possível estudar o comportamento não linear das estruturas através do método dos elementos de contorno e resolver problemas elastoplásticos e viscoplásticos, empregando-se tensões ou deformações iniciais no equacionamento do MEC. Já VENTURINI (1982 e 1984) e VENTURINI \& BREBBIA (1983 e 1984), utilizaram o método dos elementos de contorno para resolver problemas geotécnicos, considerando o comportamento plástico, viscoplástico e materiais rochosos sem resistência à tração e, também, com descontinuidades.

O método dos elementos de contorno se apresenta como uma boa opção de cálculo em problemas de domínios infinitos, semi-infinitos e regiões de grande concentração de tensões. A combinação entre os diversos métodos numéricos é um assunto de grande interesse para os pesquisadores, pois possibilita utilizar o método numérico mais conveniente a cada sub-estrutura, aproveitando melhor as particularidades de cada um. Pode-se destacar a combinação do método dos elementos finitos e elementos de contorno, que surgiu com McDONALD \& WEXLER (1972), analisando problemas de engenharia elétrica. CHEN \& MEI (1974) estudaram problemas de mecânica dos fluidos, onde o MEC foi utilizado para tratar o domínio infinito. Mas os trabalhos de ZIENKIEWICZ et al. (1977), de SHAW \& FALBY (1977), e de OSIAS et al. (1977), foram os primeiros a tratar sólidos deformáveis através da combinação elementos finitos e elementos de contorno. E AYALA \& GOMEZ (1979) apresentaram detalhes do processo de resolução de problemas elásticos tridimensionais em geomecânica.

BREBBIA \& GEORGIO (1980) analisaram problemas bidimensionais através da combinação MEC-MEF. O programa desenvolvido combina elementos de contorno constantes com elementos finitos quadráticos e, embora esta combinação não seja totalmente compatível, foram obtidos bons resultados. MUSTOE \& VOLAIT (1980) também estudaram a combinação MEC-MEF para analisar a interação túnel-suporte considerando os efeitos da descontinuidade entre rocha e concreto.

RODRÍGUEZ (1986) estudou o problema bidimensional de elasticidade linear empregando elementos contínuos e descontínuos, empregando também sistemas de resolução de equações por blocos para problemas de sub-regiões. Já ROCHA (1988) deu atenção especial aos problemas que envolvem descontinuidade, usando quadripolos e bipolos para simular as descontinuidades em problemas bi e tridimensionais, tratando o corpo em regimes elasto-plásticos ou elasto-visco-plásticos. É importante citar também que 
RAMALHO \& VENTURINI (1990) e RAMALHO (1990), analisaram no caso estático, estruturas interagindo com o meio contínuo onde o solo é modelado pelo MEC e, uma sapata rígida é utilizada como artifício para determinar as constantes de mola dos vínculos da estrutura que é tratada pelo MEF. E CODA (1993) apresentou uma formulação tridimensional dinâmica transiente para a análise específica da ligação estrutura-solo, onde a estrutura (casca, barra) é modelada pelo MEF e o solo, admitindo-se comportamento elástico linear, é modelado pelo MEC. O acoplamento da estrutura com o meio contínuo é realizado considerando-se a técnica das sub-regiões e elementos rígidos de ligação, que no caso é uma sapata rígida.

VENTURINI \& FERRO (1991 e 1992) e FERRO (1993) aplicaram a combinação MEC-MEF para analisar a interação entra estacas e o solo. As estacas são consideradas como elementos de barra modelados pelo método dos elementos finitos e, o solo como um domínio infinito, tridimensional, homogêneo, elástico linear é tratado pelo método dos elementos de contorno. Com isto, resulta um sólido infinito tridimensional enrijecido.

Em PAIVA (1991), foi apresentada uma formulação para a análise de placas elásticas em que a representação integral discretizada de Stern é alterada, de forma que a força equivalente de Kirchhoff é admitida concentrada nos pontos nodais ao longo do contorno. E em OLIVEIRA NETO \& PAIVA (1995) apresentam uma formulação a partir de alterações na representação integral de Stern, que conduz à associação de três graus de liberdade para o vetor de deslocamento, isto é, deslocamento transversal, rotação normal e rotação tangencial. Assim, para a montagem da representação algébrica são utilizadas equações integrais associadas ao deslocamento, e suas derivadas direcionais normal e tangenciais ao contorno.

KUMATSU (1995) estudou uma combinação do método dos elementos finitos (elementos uniaxias) com o método dos elementos de contorno (meios contínuos homogêneos ou não) para análise de problemas geomecânicos. AGOSTINHO (1998) emprega a técnica de sub-regiões com sub-estruturação para estudar a associação de chapas, empregando enrijecedores com auxílio do método dos elementos finitos combinado ao método dos elementos de contorno, simulando também problemas de escorregamento através de modelos plásticos e visco-plásticos simples do tipo Coulomb. Já LOPES (1996) analisa problemas de propagação de fraturas em domínios bidimensionais.

SOUZA (1999) propôs um modelo h-adaptativo para o problema físico governado pela equação de Laplace, dando especial atenção aos problemas de singularidade e hipersingularidade. 
MENDONÇA (2002) estudou o problema de laminas multiconectadas não coplanares através de duas formulações, uma chamada de tetraparamétrica e a outra hexaparamétrica, abordando problemas elásticos e elastoplásticos.

Em ARISTIDERMO \& TURCO (1994), é utilizada uma técnica de discretização em que o contorno é dividido em segmentos, então chamados macro-elementos, que possuem suas próprias funções interpoladoras. Cada um deles é dividido em elementos menores pela introdução de nós e as variáveis do contorno são interpoladas por curvas 'Spline' quadráticas. Embora as variáveis do contorno são interpoladas por funções quadráticas, as integrais são calculadas analiticamente de tal forma que os resultados são expressos em função de parcelas polinomiais de ordem genérica. Assim, interpolações com B-splines de ordens superiores, após alguns ajustes algébricos, podem ser também obtidas e empregadas em diversas análises, principalmente, envolvendo estudos de p-adaptatividade.

FOLTRAN (1999) estuda problemas planos em regime elástico e elasto-plástico pelo método dos elementos de contorno, onde ele propõe o uso de expressões analíticas para as integrais sobre o contorno para elementos isoparamétricos lineares. Inclui também expressões analíticas para o tratamento de carregamentos de domínio com células lineares.

CODA (2001) apresenta uma análise do comportamento não-linear dinâmico e estático de meios reforçados através de uma abordagem do acoplamento MEC/MEF onde o MEF é utilizado para representar os reforços no meio discretizado pelo MEC.

LEITE, CODA \& VENTURINI (2003) apresentam um estudo bidimensional de sólidos reforçados com barras usando o método dos elementos de contorno. Estes reforços feitos por fibras são feitos com sub-regiões onde é feita uma redução dos graus de liberdade do contorno através de uma aproximação linear de deslocamento da seção transversal do enrijecedor. Fazendo a integração da solução fundamental de Kelvin de forma totalmente analítica, tanto para as integrais singulares como para as não singulares.

FERNANDES \& VENTURINI (2002) e FERNANDES (2003), também aplica a técnica de redução de graus de liberdade no acoplamento MEC/MEC, para problemas em placas.

De forma semelhante, LOVÓN (2003), MACIEL (2003) obtiveram a integração totalmente analítica da solução fundamental de Kelvin, LOVÓN (2003) empregando-as para análise de erro em modelos auto-adaptativos e MACIEL (2003) utilizando também de forma analítica as equações dos gradientes de tensão para estudo de problemas de fissuras.

BOTTA \& VENTURINI (2003), tem desenvolvido o acoplamento MEC/MEF em chapas, estudando problemas de contato, na interface entre o domínio e o enrijecedor, e 
ainda para minimizar perturbações indesejáveis nos resultados nesta interface é aplicada à técnica de suavização do contorno com mínimos quadrados. Este trabalho ainda trata de problemas de localização.

Muitos programas computacionais têm sido desenvolvidos para estudo do problema elástico bidimensional, pelo método dos elementos de contorno. Contudo a maioria destes programas sempre aborda situações bem específicas, com uma interface com o usuário um tanto simplificada sendo feita de uma forma geral através de arquivos em modo texto onde todos os dados do problema devem, na maioria dos casos, serem digitados pelo usuário. Uma das propostas deste trabalho é desenvolver uma interface um pouco mais amigável com o usuário, através dum programa escrito em linguagem orientada a objeto.

Além da facilidade de operação do programa busca-se também desenvolver um algoritmo eficiente e versátil para análise de problemas elásticos. Esta eficiência é obtida através da integração analítica das funções que geram os termos tanto das matrizes de resolução do sistema quanto para as soluções fundamentais para cálculo de tensão, e também através das regularizações na montagem do sistema de equações através da implementação da técnica de mínimos quadrados. Com estas melhorias aplicadas ao modelo busca-se estudar problemas com enrijecedores empregando para isto a técnica de sub-regiões.

\subsection{Metodologia de Trabalho}

Com o intuito de tornar a interface com o usuário mais amigável e fácil de se operar, desenvolveu-se um pré-processador que interpreta dados geométricos informados a partir de um arquivo em formato "dxf".

Para processar estes dados e transforma-los em dados coerentes para o cálculo do problema elástico pelo método dos elementos de contorno, foram desenvolvidos algoritmos geradores semi-automáticos de elementos de contorno e interpretador de nós e conectividade, sendo que para a malha de domínio, acoplou-se um gerador de malha de domínio público desenvolvido por NICENO (1997).

Apesar de boa parte do programa ter sido escrita em linguagem orientada a objeto, todo o processador foi escrito em Fortran, tal escolha é conseqüência das facilidades que esta linguagem oferece no desenvolvimento de algoritmos de cálculo contendo várias bibliotecas 
de que facilitam a programação. E além do mais é uma linguagem bem difundida no meio científico, principalmente quando se refere ao método dos elementos de contorno.

Quanto ao modelo, as integrações analíticas foram desenvolvidas aplicando-se algumas técnicas de discretização das variáveis em coordenadas locais, o que facilita a dedução de tais expressões.

Buscou-se melhorar os resultados com o emprego de mais pontos fontes do que necessários escrevendo desta forma mais equações do que era necessária, sendo a redução feita através dos mínimos quadrados. Esta técnica foi empregada de duas maneiras diferentes: tanto por sub-região quanto por elemento.

Para estudo de enrijecedores, foi empregada a técnica de sub-regiões. Tal técnica é aqui viabilizada através da garantia da qualidade das equações com uso de integração analítica, podendo desta forma resolver problemas com enrijecedores bem estreitos sem perder a independência das equações do sistema.

Para análise dos resultados foi desenvolvido um pós-processador que interpreta os resultados de cálculo e gera mapas de tensão, deformação, deslocamentos e tensões principais e deformações principais. Além dos mapas de cores é possível ainda observar os resultados através de isolinhas. Tanto os mapas quanto as isolinhas podem ser sobrepostos, podendo ser visualizados na posição deformada ou não. Facilidade como aproximação (Zoom), translação (Pan), e rotação também foram implementados.

Tanto o pré-processador como o pós-processador foram desenvolvidos em linguagem computacional Delphi (Object Pascal), o gerador de malha de domínio público é conhecido como EasyMesh 1.4 desenvolvido por NICENO (1997), tem seus algoritmos escritos em linguagem $\mathrm{C}++$, todas as representações gráficas foram desenvolvidas com auxílio de comandos em OpenGL empregados no Delphi.

\subsection{OBJETIVOS DESTE TRABALHO}

Este trabalho envolvendo o Método dos Elementos de Contorno via soluções fundamentais de Kelvin para o problema bidimensional de chapas busca dar uma contribuição para três aspectos básicos que são:

Primeiramente, a melhora do modelo mecânico-computacional através do emprego da integração analítica das soluções fundamentais de Kelvin em substituição as integrais 
numéricas anteriormente empregadas, inclusive para as integrais não singulares, esta não é uma solução inédita, pois pesquisadores como Foltran, Aristidermo \& Turco, e pesquisas em desenvolvimento no departamento de estruturas de EESC dirigidas pelos professores Dr. Wilson Sérgio Venturini e Dr. Humberto Breves Coda, já desenvolveram pesquisas sobre tal assunto, contudo alguns aspectos desta técnica são complementados. Ainda sob a melhora do modelo, através da aplicação da técnica de mínimos quadrado empregado sob as sub-regiões ou sob os elementos de forma individual, obtendo-se assim resultados mais coerentes.

Um segundo aspecto a ser observado neste trabalho é no que se refere ao ganho de versatilidade da aplicação do método dos elementos de contorno com a implementação de técnicas conhecidas e muito úteis em análises como o emprego de sub-regiões e enrijecedores, sendo a primeira comumente empregada com o objetivo de simular problemas que envolvam regiões compostas de materiais diferentes, e os enrijecedores de forma semelhante para simular sub-regiões rígidas de espessura pequena, ou não.

Ainda com este trabalho buscou-se obter facilidades de utilização do método dos elementos de contorno através da criação de um pré e um pós-processador sendo o préprocessador composto de um interpretador de arquivos em formato *.dxf e ainda de interpretadores de geometria, conectividade, gerador de malha, etc. E o pós-processador empregado para visualização dos mapas de tensão coloridos ou monocromáticos, podendo ainda ser gerada curvas de iso-valores e ainda podendo apresentar a configuração deformada das estruturas simuladas. 
FUNDAMENTOS DA TEORIA DA ELASTICIDADE

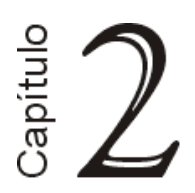

Neste capítulo são apresentados alguns conceitos básicos da teoria da elasticidade necessários para compreensão do conteúdo deste trabalho. Trata-se duma revisão sucinta, em notação indicial (apêndice A). Em 2.1 são apresentados os conceitos básicos do problema elástico. E em 2.2 são apresentadas as simplificações dos estados planos, tanto o estado plano de deformação quanto o estado plano de tensão, bem como suas particularidades.

\subsection{EquaÇões BáSICAS de TEORIA da Elasticidade}

Para as definições que serão descritas a seguir é suposto que as seguintes hipóteses básicas sejam respeitadas:

- É válida a geometria de pequenos deslocamentos;

- O estado deformado do corpo pode ser escrito em função do estado indeformado (aproximação Lagrangiana);

- O material que constitui o corpo é elástico linear, homogêneo e isotrópico.

O problema elástico, de maneira geral, fica formulado mediante 15 equações diferenciais e algébricas, são elas:

3 equações de equilíbrio;

6 relações deformação-deslocamento;

6 relações tensão-deformação.

Envolvendo assim um total de 15 funções incógnitas das variáveis independentes $\mathrm{x}$, y, z, que são: 
6 componentes de tensão;

6 componentes de deformação;

3 componentes de deslocamento.

Deve-se ainda satisfazer as condições de forças ou deslocamentos prescritos no contorno.

Pode-se resolver este problema tanto pelo método direto como pelo procedimento inverso.

No procedimento direto de resolução, integram-se as equações diferenciais que regem o problema, determinando-se a solução mediante o atendimento às condições de contorno. Se forem escolhidos como incógnitas básicas os deslocamentos, utilizam-se as equações de equilíbrio escritas em termos dos deslocamentos, mediante substituição das tensões pelas deformações, via lei de Hooke, e destas pelos deslocamentos, através das relações deformação-deslocamento. Se forem escolhidas como incógnitas básicas às tensões, as três equações de equilíbrio mostram-se insuficientes, e é necessário utilizar também as equações de compatibilidade, escritas em termos de tensões através da lei de Hooke. Em qualquer caso conforme acima observado, é ainda necessário atender às condições no contorno do sólido.

É comum adotar-se o método inverso: onde a forma de solução (usualmente em termos de tensão) é fixada a priori, atendendo às condições de equilíbrio e conduzindo a um campo de deformações compatível; determina-se então as forças de superfície correspondentes, pelas condições de contorno. Outra possibilidade seria fixar o campo de deslocamentos (atendendo às equações de equilíbrio escritas em termos destes), determinando-se então as deformações e tensões (pelas relações deformação-deslocamento e pela lei de Hooke), e finalmente as forças no contorno.

\subsubsection{Equações diferenciais de equilíbrio (Navier)}

Em um sólido em equilíbrio sob a ação de um sistema de forças, seja um paralelepípedo infinitesimal de arestas dx, dy, dz paralelas aos eixos coordenados. (Fig. 01) 


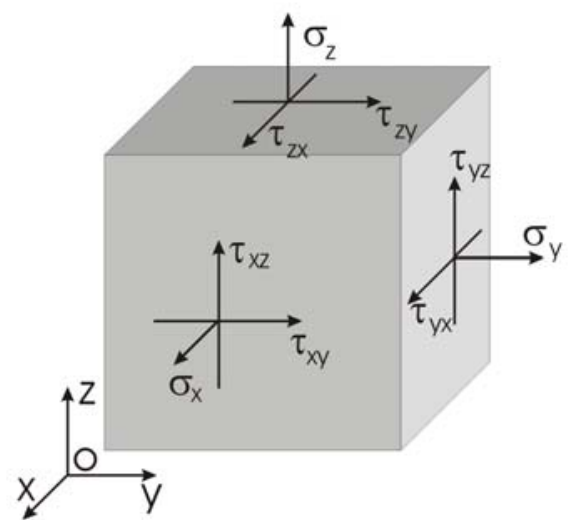

Figura 01 - (a) Elemento infinitesimal.

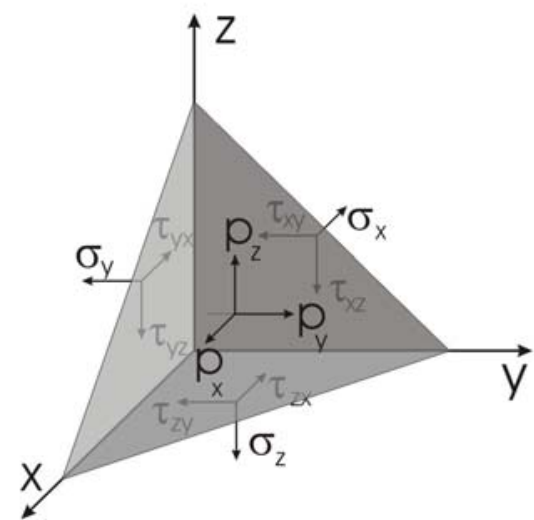

(b) Forças de superfície

Sabe-se que as forças externas atuando em um corpo podem ser distribuídas no volume ou na superfície, onde se observa que as forças de volume atuam interiormente ao corpo, como a gravidade, por exemplo. As equações de equilíbrio podem ser escritas, em notação indicial, da seguinte forma:

$$
\begin{aligned}
& \sigma_{i j, j}+b_{i}=0 \quad(i, j=1,3) . \\
& p_{i}=\sigma_{j i} \eta_{j}
\end{aligned}
$$

onde

$\sigma_{\mathrm{ij}}$ ou $\sigma_{\mathrm{ji}}$ são tensões internas

$b_{i}$ São forças externas por unidade de volume

$\mathrm{p}_{\mathrm{i}}$ São forças externas por unidade de superfície

Quando não há momentos por unidade de volume, resulta:

$$
\sigma_{\mathrm{ij}}=\sigma_{\mathrm{ji}}
$$




\subsubsection{Relações deformação - deslocamento}

Admitindo-se as hipóteses de continuidade e que pontos vizinhos no corpo permanecem na vizinhança após este ser deformado, chega-se a definir o tensor deformação, que referido ao mesmo sistema cartesiano de coordenadas ortogonais, tanto para a configuração original quanto para a deformada, é dado por:

$\varepsilon_{\mathrm{ij}}=1 / 2\left(\mathrm{u}_{\mathrm{j}, \mathrm{i}}+\mathrm{u}_{\mathrm{i}, \mathrm{j}}-\mathrm{u}_{\mathrm{k}, \mathrm{i}} \mathrm{u}_{\mathrm{k}, \mathrm{j}}\right)$

Restringindo-se apenas ao estudo das pequenas rotações, podem-se desprezar infinitésimos de ordem superior como o último termo da eq.(2.3). As componentes de deformação podem então ser escritas assim:

$\varepsilon_{\mathrm{ij}}=1 / 2\left(\mathrm{u}_{\mathrm{j}, \mathrm{i}}+\mathrm{u}_{\mathrm{i}, \mathrm{j}}\right)$

\subsubsection{Equações de compatibilidade de deformações}

Estas equações garantem condições para que, fixada os seis componentes de deformação, seja possível garantir a integridade das relações deformação-deslocamento. Estas relações suplementares são obtidas, por exemplo, através de alguns cálculos eliminando-se as componentes de deslocamento das relações deformação - deslocamento, obtendo-se assim o conjunto de seis equações independentes de compatibilidade de deformações:

$$
\varepsilon_{\mathrm{ij}, \mathrm{kl}}+\varepsilon_{\mathrm{kl}, \mathrm{jj}}-\varepsilon_{\mathrm{ik}, \mathrm{jl}}-\varepsilon_{\mathrm{jl}, \mathrm{ik}}=0
$$

\subsubsection{Equações constitutivas - Lei de Hooke generalizada}

$\mathrm{Na}$ teoria da elasticidade define-se a relação entre o tensor de tensão e o tensor de deformação pela chamada lei de Hooke generalizada:

$$
\sigma_{\mathrm{ij}}=\mathrm{C}_{\mathrm{ijkl}} \varepsilon_{\mathrm{kl}}
$$


onde $\mathrm{C}_{\mathrm{ijkl}}$ é um tensor de quarta ordem que contém as constantes que caracterizam o material no âmbito elástico.

Considerando-se a simetria dos tensores de tensão e de deformação, o principio de conservação de energia, e a suposição do material ser isótropo (material que possui as mesmas características elásticas em qualquer direção) reduz-se o número de constantes elásticas a duas, em geral definidas por:

$\mathrm{E}=$ Módulo de elasticidade Longitudinal (Modulo de Young);

$v=$ Coeficiente de Poisson.

Assim, pode-se escrever a relação entre tensão e deformação como sendo:

$$
\begin{array}{r}
\sigma_{\mathrm{ij}}=2 \mathrm{G} \varepsilon_{\mathrm{ij}}+\frac{2 \mathrm{G} v}{1-2 v} \varepsilon_{\mathrm{kk}} \delta_{\mathrm{ij}} \\
\text { onde } \mathrm{G}=\frac{\mathrm{E}}{2(1+v)}
\end{array}
$$

é o módulo de elasticidade transversal, também chamado de módulo de elasticidade ao cisalhamento.

Pode-se escrever também a relação inversa da seguinte forma:

$$
\varepsilon_{\mathrm{ij}}=\frac{1}{2 \mathrm{G}}\left[\sigma_{\mathrm{ij}}-\frac{v}{1-v} \sigma_{\mathrm{kk}} \delta_{\mathrm{ij}}\right]
$$

\subsubsection{Condições de Contorno}

$\mathrm{Na}$ formulação do problema elástico, além das equações que devem ser satisfeitas no domínio, outras condições devem ser atendidas no contorno do sólido. De maneira geral pode-se ter: ou deslocamentos ou forças prescritas no contorno (apêndice B). 


\subsubsection{Estados Planos}

Em determinadas situações pode-se adotar simplificações para representar um determinado problema elástico, caindo assim num problema de estado plano de tensão ou de deformação dependendo de certas característica existentes no problema real.

Quando uma das dimensões do corpo for muito maior que as outras duas, e o carregamento é unicamente perpendicular aos elementos longitudinais e não variam ao longo do comprimento, pode-se supor que todas as seções transversais estão com as mesmas condições. De tal forma que o deslocamento na direção axial é impedido. As componentes u e v do deslocamento são funções de x e y, mas são independentes da coordenada longitudinal

z. Ficando o estado de deformação especificado apenas por $\varepsilon_{\mathrm{x}}, \varepsilon_{\mathrm{y}}$ e $\gamma_{\mathrm{xy}}$ que é denominado estado plano de deformação, sendo esta três componentes funções somente de x e y.

As relações constitutivas para este caso têm a mesma expressão de (2.6), no entanto os índices variam apenas até 2 . As tensões tangenciais referentes à direção $\mathrm{x}_{3}$ serão nulas $\mathrm{e} \mathrm{a}$ tensão normal $\sigma_{33}$ tem seu valor expresso apenas em função de $\sigma_{11}$ e de $\sigma_{22}$.

Quando uma das dimensões do corpo for muito menor que as outras duas, e o carregamento é unicamente no plano destas, pode-se supor que as tensões ao longo da terceira direção são nulas. Ficando o estado de tensão especificado apenas por $\sigma_{\mathrm{x}}, \sigma_{\mathrm{y}} \mathrm{e} \tau_{\mathrm{xy}}$ que é denominado estado plano de tensão. Sendo admitido estas três componentes serem independentes de $\mathrm{z}$, elas não variam ao longo da espessura sendo funções somente de $\mathrm{x}$ e $\mathrm{y}$. Para este caso (estado plano de tensão) as relações podem ser obtidas a partir das relações do estado plano de deformação fazendo-se as seguintes transformações:

$$
\begin{aligned}
& v^{\prime}=\frac{v}{1+v} \\
& G^{\prime}=G
\end{aligned}
$$




\section{SOLUÇÃo Fundamental DE KELVIN E EQUAÇÃo Integral Para o Problema Elástico Bidimensional

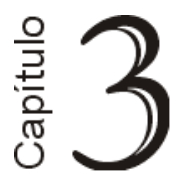

Neste capítulo são apresentadas as equações integrais de contorno, bem como a solução fundamental de Kelvin.

\subsection{Métodos}

A formulação do Método dos Elementos de Contorno pode ser obtida de diversas formas, como por exemplo, através do teorema da reciprocidade de Betti, introduzindo alguns conceitos de resíduos ponderados, ou até mesmo utilizando uma formulação variacional semelhante à empregada no Método dos Elementos Finitos. Neste trabalho será descrita a formulação baseada na reciprocidade de Betti.

Pode-se dividir as formulações do Método dos Elementos de Contorno em três categorias:

Método Indireto: baseado na utilização de funções-densidade fictícias para formular o problema. Embora essas funções não tenham significado físico definido, podem ser integradas para obtenção de deslocamentos e tensões. Essa abordagem é dita indireta, pois os deslocamentos e tensões reais não são utilizados em toda a formulação.

Método Semidireto: as funções empregadas neste caso para formular o problema estão relacionadas às funções de tensão que tem maior significado físico do que as funçõesdensidade fictícia, podendo as funções de tensão serem diferenciadas ou integradas obtendose assim deslocamentos e tensões.

Método Direto: neste método, grandezas físicas como, deslocamentos e forças de superfície, são utilizadas desde o início do desenvolvimento das equações integrais. As 
equações que regem o problema, relações constitutivas e equações de equilíbrio são transformadas em equações integrais válidas no contorno através dos teoremas de Green e do teorema da reciprocidade de Betti.

\subsection{SOLUÇão FUndAMENTAL dE KELVIN}

Antes da definição de solução fundamental é necessário definir que se está tratando de um meio elástico linear no qual se aplica uma força unitária no ponto s, chamado de ponto fonte (source point) e mede-se o efeito desta carga unitária no ponto q. O domínio do problema é infinito, sempre que se desejar referir ao problema fundamental será colocado o asterisco.

Entende-se por solução fundamental de Kelvin, a solução para o problema elástico cujo carregamento é dito unitário, ou seja, na equação de equilíbrio (equação de Navier) substitui-se a parcela referente à força de volume b bela função Delta de Dirac (anexo B).

$$
\mathrm{b}_{\mathrm{i}}(\mathrm{q})=\delta(\mathrm{s}, \mathrm{q}) \mathrm{c}_{\mathrm{i}}(\mathrm{s})
$$

onde $\mathrm{c}_{\mathrm{i}}(\mathrm{s})$ trata-se do co-seno do ângulo entre a força e o eixo $\mathrm{x}_{\mathrm{i}}$ que pode ser reescrito tomando-se os devidos cuidados com a função delta de Kronecker $\delta_{\text {ik }}$ (anexo C).

Ficando a equação de equilíbrio desta maneira:

$$
\sigma_{\mathrm{kij}, \mathrm{j}}^{*}+\delta(\mathrm{s}, \mathrm{q}) \delta_{\mathrm{ki}}=0
$$

Substituindo na Lei de Hooke (para o problema fundamental) a relação deformação deslocamento eq.(2.4) e em seguida derivando-se com respeito à $\mathrm{x}_{\mathrm{j}}$ e substituindo-se o resultado na eq.(3.2), tem-se:

$$
\frac{1}{1-2 v} u_{\mathrm{kj}, \mathrm{ij}}^{*}+\mathrm{u}_{\mathrm{ki}, \mathrm{jj}}^{*}+\frac{\delta(\mathrm{s}, \mathrm{q}) \delta_{\mathrm{ki}}}{\mathrm{G}}=0
$$

Uma solução desta eq.(3.3) é para a elasticidade plana dada por: 


$$
\mathrm{u}_{\mathrm{ij}}^{*}(\mathrm{~s}, \mathrm{q})=\frac{1}{8 \pi \mathrm{G}(1-v)}\left[(3-4 v) \ln \left(\frac{1}{\mathrm{r}}\right) \delta_{\mathrm{ij}}+\mathrm{r}_{, \mathrm{i}} \mathrm{r}_{\mathrm{j}}\right]
$$

Onde o primeiro índice refere-se à direção de aplicação da carga unitária e o segundo índice à direção do deslocamento correspondente.

Para o problema tridimensional a equação (3.4) é escrita desta forma:

$$
\mathrm{u}_{\mathrm{ij}}^{*}(\mathrm{~s}, \mathrm{q})=\frac{1}{16 \pi \mathrm{G}(1-v) \mathrm{r}}\left[(3-4 v) \delta_{\mathrm{ij}}+\mathrm{r}_{, \mathrm{i}} \mathrm{r}_{\mathrm{j}}\right]
$$

Derivando-se a equação eq.(3.4) com respeito à $\mathrm{x}_{\mathrm{k}}$, rearranjando-se e substituindo-se na relação deformação deslocamento eq.(2.4) chega-se para o caso bidimensional à:

$$
\varepsilon_{\mathrm{ijk}}^{*}(\mathrm{~s}, \mathrm{q})=\frac{-1}{8 \pi \mathrm{G}(1-v) \mathrm{r}}\left[(1-2 v)\left(\mathrm{r}_{, \mathrm{k}} \delta_{\mathrm{ij}}+\mathrm{r}_{\mathrm{j} j} \delta_{\mathrm{ik}}\right)-\mathrm{r}_{\mathrm{i}} \delta_{\mathrm{jk}}+2 \mathrm{r}_{, \mathrm{i}, \mathrm{j}, \mathrm{r}} \mathrm{r}_{\mathrm{k}}\right]
$$

$\mathrm{Na}$ forma geral tem-se:

$$
\varepsilon_{\mathrm{ijk}}^{*}(\mathrm{~s}, \mathrm{q})=\frac{-1}{8 \alpha \pi \mathrm{G}(1-v) \mathrm{r}^{\alpha}}\left[(1-2 v)\left(\mathrm{r}_{, \mathrm{k}} \delta_{\mathrm{ij}}+\mathrm{r}_{\mathrm{j}} \delta_{\mathrm{ik}}\right)-\mathrm{r}_{, \mathrm{i}} \delta_{\mathrm{jk}}+\beta \mathrm{r}_{\mathrm{i}, \mathrm{r}, \mathrm{r}, \mathrm{r}}\right]
$$

Onde

$$
\left\{\begin{array}{l}
\text { Caso 2D } \Rightarrow \alpha=1, \beta=2 \\
\text { Caso 3D } \Rightarrow \alpha=2, \beta=3
\end{array}\right.
$$

Aplicando-se a eq.(3.7) na lei de Hooke pode-se obter, para o caso bidimensional:

$$
\sigma_{\mathrm{ijk}}^{*}(\mathrm{~s}, \mathrm{q})=\frac{-1}{4 \pi(1-v) \mathrm{r}}\left[(1-2 v)\left(\mathrm{r}_{, \mathrm{k}} \delta_{\mathrm{ij}}+\mathrm{r}_{\mathrm{j}} \delta_{\mathrm{ik}}-\mathrm{r}_{, \mathrm{i}} \delta_{\mathrm{jk}}\right)+2 \mathrm{r}_{\mathrm{i}, \mathrm{j}, \mathrm{r}, \mathrm{r}}\right]
$$

$\mathrm{Na}$ forma geral tem-se:

$$
\sigma_{\mathrm{ijk}}^{*}(\mathrm{~s}, \mathrm{q})=\frac{-1}{4 \alpha \pi(1-v) \mathrm{r}^{\alpha}}\left[(1-2 v)\left(\mathrm{r}_{, \mathrm{k}} \delta_{\mathrm{ij}}+\mathrm{r}_{\mathrm{j}} \delta_{\mathrm{ik}}-\mathrm{r}_{\mathrm{i}} \delta_{\mathrm{jk}}\right)+\beta \mathrm{r}_{\mathrm{i}, \mathrm{r}, \mathrm{j}, \mathrm{r}}\right]
$$


E por fim aplicando-se esta na fórmula de Cauchy, obtém-se para o caso bidimensional:

$$
\mathrm{P}_{\mathrm{ij}}^{*}(\mathrm{~s}, \mathrm{q})=\frac{-1}{4 \pi(1-v) \mathrm{r}}\left\{\left[(1-2 v) \delta_{\mathrm{ij}}+2 \mathrm{r}_{, \mathrm{i}, \mathrm{j}} \mathrm{r}_{\mathrm{j}}\right] \mathrm{r}_{\mathrm{n}}-(1-2 v)\left(\mathrm{r}_{, \mathrm{i}} \mathrm{n}_{\mathrm{j}}-\mathrm{r}_{, \mathrm{j}} \mathrm{n}_{\mathrm{i}}\right)\right\}
$$

$\mathrm{Na}$ forma geral tem-se:

$$
\mathrm{P}_{\mathrm{ij}}^{*}(\mathrm{~s}, \mathrm{q})=\frac{-1}{4 \alpha \pi(1-v) \mathrm{r}^{\alpha}}\left\{\left[(1-2 v) \delta_{\mathrm{ij}}+\beta \mathrm{r}_{, \mathrm{i}} \mathrm{r}_{\mathrm{j}}\right] \mathrm{r}_{, \mathrm{n}}-(1-2 v)\left(\mathrm{r}_{, \mathrm{i}} \mathrm{n}_{\mathrm{j}}-\mathrm{r}_{, \mathrm{j}} \mathrm{n}_{\mathrm{i}}\right)\right\}
$$

\subsection{EquaÇão Integral de Contorno Para o Problema Elástico Plano}

São apresentadas nesta seção as representações integrais básicas de um corpo elástico, que são ferramentas básicas para resolução de problemas pelo método dos elementos de contorno. Estas fórmulas serão deduzidas para o problema de estado plano de deformação, supondo que não exista descontinuidade no corpo.

Seja um domínio $\Omega$, limitado por um contorno $\Gamma$, submetido a dois estados de carregamento: sendo o primeiro o problema em estudo e o segundo, o problema de Kelvin. Através do teorema da reciprocidade de Betti pode-se obter a seguinte igualdade:

$$
\int_{\Omega} \sigma_{\mathrm{jk}}(\mathrm{q}) \varepsilon_{\mathrm{ijk}}^{*}(\mathrm{~s}, \mathrm{q}) \mathrm{d} \Omega=\int_{\Omega} \sigma_{\mathrm{ijk}}^{*}(\mathrm{~s}, \mathrm{q}) \varepsilon_{\mathrm{jk}}(\mathrm{q}) \mathrm{d} \Omega
$$

Onde os termos que contém * estão relacionando as variáveis do problema fundamental de Kelvin e as que não contém tal símbolo representam o problema real.

Manipulando-se a expressão (3.12) com auxílio da relação deformação deslocamento, eq. (2.4), pode-se obter a seguinte relação:

$$
\int_{\Omega} \sigma_{\mathrm{jk}} \mathrm{u}_{\mathrm{ij}, \mathrm{k}}^{*} \mathrm{~d} \Omega=\int_{\Omega} \sigma_{\mathrm{ijk}}^{*} \mathrm{u}_{\mathrm{j}, \mathrm{k}} \mathrm{d} \Omega
$$

Integrando-se por partes em ambos os termos da expressão (3.13), chega-se a expressão: 


$$
-\int_{\Omega} \sigma_{\mathrm{jk}, \mathrm{k}} \mathrm{u}_{\mathrm{ij}}^{*} \mathrm{~d} \Omega+\int_{\Gamma} \sigma_{\mathrm{jk}} \mathrm{u}_{\mathrm{ij}}^{*} \mathrm{n}_{\mathrm{k}} \mathrm{d} \Gamma=-\int_{\Omega} \sigma_{\mathrm{ijk}, \mathrm{k}}^{*} \mathrm{u}_{\mathrm{j}} \mathrm{d} \Omega+\int_{\Gamma} \sigma_{\mathrm{ijk}}^{*} \mathrm{u}_{\mathrm{j}} \mathrm{n}_{\mathrm{k}} \mathrm{d} \Gamma
$$

Substituindo na expressão (3.14) a equação de Cauchy tanto no problema real como no fundamental

$$
\left\{\begin{array}{c}
P_{j}=\sigma_{j k} n_{k} \\
P_{i k}^{*}=\sigma_{i j k}^{*} n_{k}
\end{array}\right.
$$

Pode-se obter:

$$
-\int_{\Omega} \sigma_{\mathrm{jk}, \mathrm{k}} \mathrm{u}_{\mathrm{ij}}^{*} \mathrm{~d} \Omega+\int_{\Gamma} \mathrm{P}_{\mathrm{j}} \mathrm{u}_{\mathrm{ij}}^{*} \mathrm{~d} \Gamma=-\int_{\Omega} \sigma_{\mathrm{ijk}, \mathrm{k}}^{*} \mathrm{u}_{\mathrm{j}} \mathrm{d} \Omega+\int_{\Gamma} \mathrm{u}_{\mathrm{j}} \mathrm{P}_{\mathrm{ij}}^{*} \mathrm{~d} \Gamma
$$

E ao empregar-se na expressão (3.15) a equação de equilíbrio tanto do problema real como do fundamental

$$
\left\{\begin{array}{c}
\sigma_{j k, k}=-b_{j} \\
\sigma_{i j k, k}^{*}=-\delta(s, q) \delta_{i j}
\end{array}\right.
$$

Chega-se à:

$$
\int_{\Omega} \mathrm{b}_{\mathrm{j}} \mathrm{u}_{\mathrm{ij}}^{*} \mathrm{~d} \Omega+\int_{\Gamma} \mathrm{P}_{\mathrm{j}} \mathrm{u}_{\mathrm{ij}}^{*} \mathrm{~d} \Gamma=\int_{\Omega} \delta(\mathrm{s}, \mathrm{q}) \delta_{\mathrm{ij}} \mathrm{u}_{\mathrm{j}} \mathrm{d} \Omega+\int_{\Gamma} \mathrm{u}_{\mathrm{j}} \mathrm{P}_{\mathrm{ij}}^{*} \mathrm{~d} \Gamma
$$

Rearranjando e resolvendo a integral $\delta_{\mathrm{ij}} \mathrm{u}_{\mathrm{j}} \int \delta(\mathrm{s}, \mathrm{q}) \mathrm{d} \Omega=\delta_{\mathrm{ij}} \mathrm{u}_{\mathrm{j}}$, tem-se:

$$
\int_{\Gamma} \mathrm{P}_{\mathrm{j}} \mathrm{u}_{\mathrm{ij}}^{*} \mathrm{~d} \Gamma-\int_{\Gamma} \mathrm{u}_{\mathrm{j}} \mathrm{P}_{\mathrm{ij}}^{*} \mathrm{~d} \Gamma+\int_{\Omega} \mathrm{b}_{\mathrm{j}} \mathrm{u}_{\mathrm{ij}}^{*} \mathrm{~d} \Omega=\delta_{\mathrm{ij}} \mathrm{u}_{\mathrm{j}}
$$

Como $\delta_{\mathrm{ij}} \mathrm{u}_{\mathrm{j}}=\mathrm{u}_{\mathrm{i}}$ tem-se:

$$
u_{i}(s)=-\int_{\Gamma} P_{i j}^{*}(s, q) u_{j}(q) d \Gamma+\int_{\Gamma} u_{i j}^{*}(s, q) P_{j}(q) d \Gamma+\int_{\Omega} b_{j}(q) u_{i j}^{*}(s, q) d \Omega
$$

$$
\text { Lembrando que para } \mathrm{s} \notin \Omega \Rightarrow \mathrm{u}_{\mathrm{i}}(\mathrm{s})=0 \text {. }
$$


Aplicando-se a equação (3.18) na Lei de Hooke chega-se a equação integral de tensões para pontos internos:

$$
\begin{aligned}
\sigma_{\mathrm{ij}}(\mathrm{s})= & -\int_{\Gamma}\left[\frac{2 \mathrm{G} v}{1-2 v} \delta_{\mathrm{ij}} \mathrm{P}_{\mathrm{lk}, \mathrm{l}}^{*}+\mathrm{G}\left(\mathrm{P}_{\mathrm{ik}, \mathrm{j}}^{*}+\mathrm{P}_{\mathrm{jk}, \mathrm{i}}^{*}\right)\right] \mathrm{u} \mathrm{k} \mathrm{d} \Gamma \\
& +\int_{\Gamma}\left[\frac{2 \mathrm{G} v}{1-2 v} \delta_{\mathrm{ij}} \mathrm{u}_{\mathrm{lk}, \mathrm{l}}^{*}+\mathrm{G}\left(\mathrm{u}_{\mathrm{ik}, \mathrm{j}}^{*}+\mathrm{u}_{\mathrm{jk}, \mathrm{i}}^{*}\right)\right] \mathrm{P}_{\mathrm{k}} \mathrm{d} \Gamma \\
& +\int_{\Omega}\left[\frac{2 \mathrm{G} v}{1-2 v} \delta_{\mathrm{ij}} \mathrm{u}_{\mathrm{lk}, 1}^{*}+\mathrm{G}\left(\mathrm{u}_{\mathrm{ik}, \mathrm{j}}^{*}+\mathrm{u}_{\mathrm{jk}, \mathrm{i}}^{*}\right)\right] \mathrm{b}_{\mathrm{k}} \mathrm{d} \Omega
\end{aligned}
$$

\section{Equação integral para pontos do contorno}

É conhecido que a equação Somigliana pode fornecer valores de deslocamentos em qualquer ponto interno, desde que os valores de forças e deslocamentos sejam conhecidos em todos os pontos do contorno. Como a equação de Somigliana é válida para qualquer ponto do domínio $\Omega$, incluindo-se o contorno $\Gamma$, é possível encontrar uma equação integral levando-se o ponto de colocação para o contorno. Para isso, é necessário, quando o ponto fonte está no contorno, retirar a singularidade através de alguns cálculos, organizando a equação Somigliana agora sem singularidade particularizada para o ponto fonte pertencente ao contorno.

Se o ponto fonte estiver sobre um trecho do contorno dito suave, é possível suplementar o domínio com um hemisfério de raio $\varepsilon$, centrado no ponto fonte. Fazendo o raio $\varepsilon$ tender a zero, o ponto fonte torna-se um ponto do contorno e a expressão resultante será a equação Somigliana particularizada para o ponto fonte situado no contorno. Como é apresentado na figura abaixo:

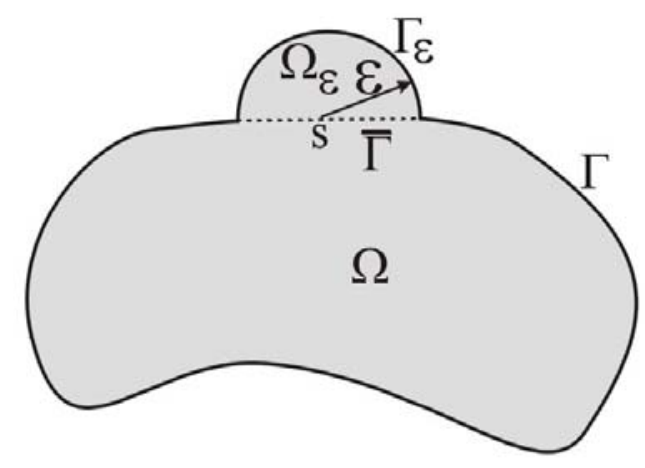

Figura 02 - Solução para singularidade em ponto fonte no contorno. 
Inserindo-se o semicírculo a equação Somigliana e aplicando o limite quando $\varepsilon$ tende a zero, tem-se:

$$
\begin{aligned}
\mathrm{u}_{\mathrm{i}}(\mathrm{s})=\lim _{\varepsilon \rightarrow 0}[ & -\int_{\Gamma-\bar{\Gamma}+\Gamma_{\varepsilon}} \mathrm{P}_{\mathrm{ij}}^{*}(\mathrm{~s}, \mathrm{q}) \mathrm{u}_{\mathrm{j}}(\mathrm{q}) \mathrm{d} \Gamma+\int_{\Gamma-\bar{\Gamma}+\Gamma_{\varepsilon}} \mathrm{u}_{\mathrm{ij}}^{*}(\mathrm{~s}, \mathrm{q}) \mathrm{P}_{\mathrm{j}}(\mathrm{q}) \mathrm{d} \Gamma \\
& \left.+\int_{\Omega+\Omega_{\varepsilon}} \mathrm{b}_{\mathrm{j}}(\mathrm{q}) \mathrm{u}_{\mathrm{ij}}^{*}(\mathrm{~s}, \mathrm{q}) \mathrm{d} \Omega\right]
\end{aligned}
$$

Como:

$$
\begin{aligned}
& \lim _{\varepsilon \rightarrow 0}\left[\int_{\Omega_{\varepsilon}} b_{j}(q) u_{i j}^{*}(s, q) d \Omega\right]=0 \\
& \lim _{\varepsilon \rightarrow 0}\left[\int_{\Gamma_{\varepsilon}} u_{i j}^{*}(s, q) P_{j}(q) d \Gamma\right]=0
\end{aligned}
$$

$$
\lim _{\varepsilon \rightarrow 0}\left[-\int_{\Gamma-\bar{\Gamma}} P_{i j}^{*}(s, q) u_{j}(q) d \Gamma\right]=-\int_{\Gamma} P_{i j}^{*}(s, q) u_{j}(q) d \Gamma
$$

$$
\lim _{\varepsilon \rightarrow 0}\left[\int_{\Gamma-\bar{\Gamma}} u_{i j}^{*}(s, q) P_{j}(q) d \Gamma\right]=\int_{\Gamma} u_{i j}^{*}(s, q) P_{j}(q) d \Gamma
$$

$$
\lim _{\varepsilon \rightarrow 0}\left[\int_{\Omega} b_{j}(q) u_{i j}^{*}(s, q) d \Omega\right]=\int_{\Omega} b_{j}(q) u_{i j}^{*}(s, q) d \Omega
$$

$$
\begin{aligned}
\lim _{\varepsilon \rightarrow 0}\left[-\int_{\Gamma_{\varepsilon}} P_{i j}^{*}(s, q) u_{j}(q) d \Gamma\right] & =\lim _{\varepsilon \rightarrow 0}\left[-\int_{\Gamma_{\varepsilon}} P_{i j}^{*}(s, q)\left(u_{j}(q)-u_{j}(s)\right) d \Gamma\right] \\
& +\lim _{\varepsilon \rightarrow 0}\left[-\int_{\Gamma_{\varepsilon}} P_{i j}^{*}(s, q)\left(u_{j}(s)\right) d \Gamma\right]
\end{aligned}
$$

Neste ultimo limite, pode-se aplicar a condição de Hölder e obter:

$$
\lim _{\varepsilon \rightarrow 0}\left[-\int_{\Gamma_{\varepsilon}} P_{i j}^{*}(s, q) u_{j}(q) d \Gamma\right]=+\lim _{\varepsilon \rightarrow 0}\left[-u_{j}(s) \int_{\Gamma_{\varepsilon}} P_{i j}^{*}(s, q) d \Gamma\right]
$$

Chegando-se assim a equação Somigliana modificada para o contorno:

$$
c_{i j}(s) u_{j}(s)=-\int_{\Gamma} P_{i j}^{*}(s, q) u_{j}(q) d \Gamma+\int_{\Gamma} u_{i j}^{*}(s, q) P_{j}(q) d \Gamma+\int_{\Omega} b_{j}(q) u_{i j}^{*}(s, q) d \Omega
$$

Para contorno suave segundo a figura $03 \mathrm{c}_{\mathrm{ij}}(\mathrm{s})$ assume os seguintes valores: 
$\mathrm{c}_{\mathrm{ij}}(\mathrm{s}) \mathrm{u}_{\mathrm{j}}(\mathrm{s})=\frac{1}{2} \delta_{\mathrm{ij}} \mathrm{u}_{\mathrm{j}}(\mathrm{s})$

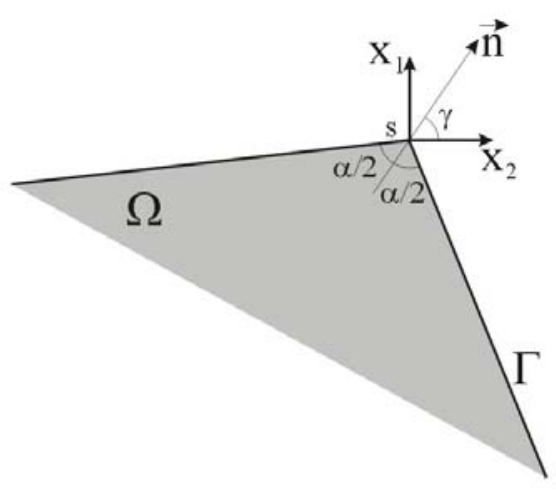

(a)Ponto Fonte em um Ponto de Contorno Com Angulosidade

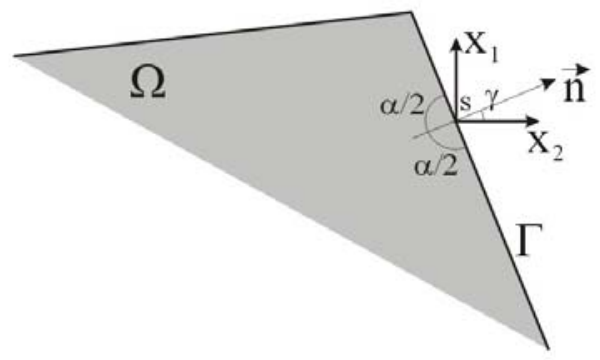

(b) Ponto Fonte em um Ponto de Contorno Suave

Figura 03 - Ponto fonte no contorno, classificação para definição de $\mathrm{c}_{\mathrm{ij}}(\mathrm{s})$.

E para contorno com angulosidade segundo a figura $03 \mathrm{c}_{\mathrm{ij}}(\mathrm{s})$ assume os seguintes valores:

$c_{i j}(s) u_{j}(s)=\left[\begin{array}{cc}\frac{\alpha}{2 \pi}+\frac{\cos 2 \gamma \operatorname{sen} \alpha}{4 \pi(1-v)} & \frac{\operatorname{sen} 2 \gamma \operatorname{sen} \alpha}{4 \pi(1-v)} \\ \frac{\operatorname{sen} 2 \gamma \operatorname{sen} \alpha}{4 \pi(1-v)} & \frac{\alpha}{2 \pi}+\frac{\cos 2 \gamma \operatorname{sen} \alpha}{4 \pi(1-v)}\end{array}\right] u_{j}(s)$ 


\section{Método dos Elementos de Contorno}

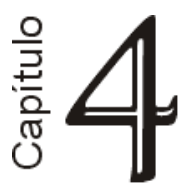

\subsection{EQUAÇÃo ALGÉBRICA}

Para que as equações integrais possam ser resolvidas, é necessário discretizarmos o contorno em elementos de forma conhecida. É importante lembrar que quando falamos de aproximação do contorno em elementos, estamos falando de dois tipos distintos de aproximação: Função de aproximação das variáveis do problema e função de aproximação da geometria do contorno.
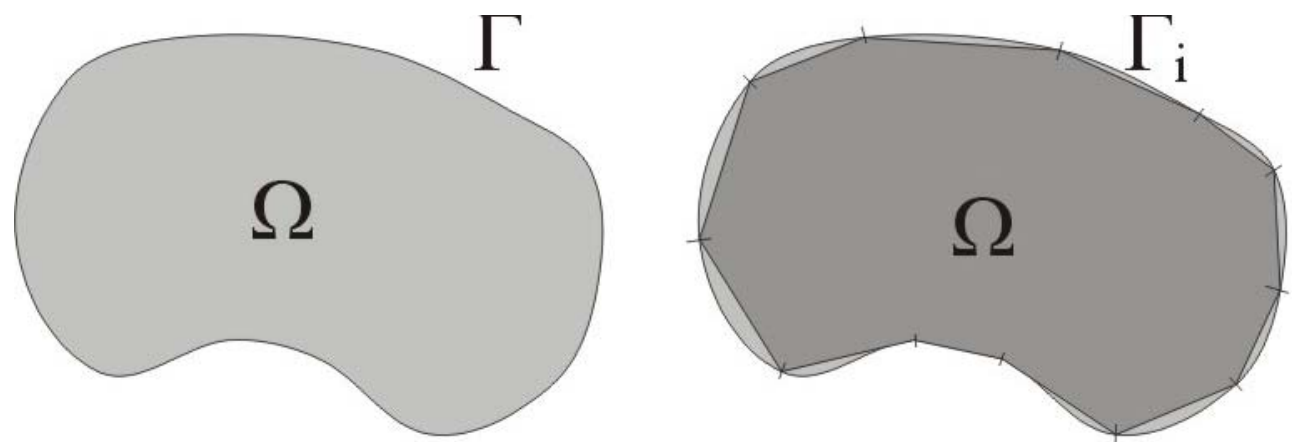

Figura 04 - Discretização do contorno em elementos.

De acordo com o grau de aproximação, os elementos podem ser classificados em constantes, lineares, quadráticos, cúbicos ou de ordem superior. Além disso, é possível admitir que o grau de aproximação de geometria e variáveis seja diferente ou não, gerando assim uma outra classificação em elementos subparamétricos, isoparamétricos ou superparamétricos. 
Neste trabalho serão empregados apenas elementos lineares isoparamétricos. Desconsiderando-se as forças de volume e discretizando-se a eq.(3.28) obtemos a seguinte expressão:

$$
[\mathrm{c}]\{\mathrm{u}\}^{\mathrm{p}}+\sum_{\mathrm{j}=1}^{\mathrm{ne}}\left(\int_{\Gamma_{\mathrm{j}}}\left[\mathrm{p}^{*}\right]\{\mathrm{u}\} \mathrm{d} \Gamma_{\mathrm{j}}\right)=\sum_{\mathrm{j}=1}^{\mathrm{ne}}\left(\int_{\Gamma_{\mathrm{j}}}\left[\mathrm{u}^{*}\right]\{\mathrm{p}\} \mathrm{d} \Gamma_{\mathrm{j}}\right)
$$

Onde ne representa o número de elementos de contorno utilizados para discretizá-lo.

\subsubsection{Funções aproximadoras}

Neste trabalho as funções aproximadoras das variáveis são lineares, tal aproximação pode ser feita empregando-se duas funções de interpolação $\phi_{1}$ e $\phi_{2}$, definidas em termos da coordenada homogênea $\xi$ como é apresentado na figura 05 .
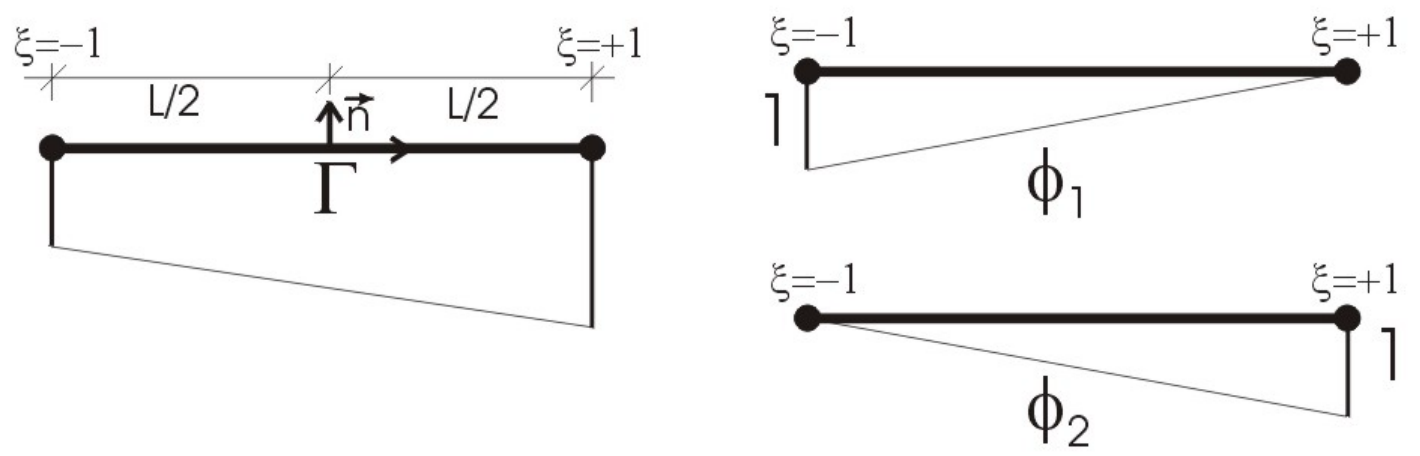

Figura 05 - Elemento linear isoparamétrico.

Logo podemos escrever que:

$\{u\}=\left\{\begin{array}{l}u_{1} \\ u_{2}\end{array}\right\}=\left[\begin{array}{cccc}\phi_{1} & 0 & \phi_{2} & 0 \\ 0 & \phi_{1} & 0 & \phi_{2}\end{array}\right]\left\{\begin{array}{l}u_{1}^{1} \\ u_{2}^{1} \\ u_{1}^{2} \\ u_{2}^{2}\end{array}\right\}=[\Phi]\{u\}_{n}^{j}$ 


$$
\{\mathrm{p}\}=\left\{\begin{array}{l}
\mathrm{p}_{1} \\
\mathrm{p}_{2}
\end{array}\right\}=\left[\begin{array}{cccc}
\phi_{1} & 0 & \phi_{2} & 0 \\
0 & \phi_{1} & 0 & \phi_{2}
\end{array}\right]\left\{\begin{array}{l}
\mathrm{p}_{1}^{1} \\
\mathrm{p}_{2}^{1} \\
\mathrm{p}_{1}^{2} \\
\mathrm{p}_{2}^{2}
\end{array}\right\}=[\Phi]\{\mathrm{p}\}_{\mathrm{n}}^{\mathrm{j}}
$$

Onde $u_{w}^{k}\left(p_{w}^{k}\right)$ representa o deslocamento (força de superfície), na direção w, do nó $\mathrm{k}$ do elemento e $\{\mathrm{u}\}_{\mathrm{w}}^{\mathrm{k}}\left(\{\mathrm{p}\}_{\mathrm{w}}^{\mathrm{k}}\right)$ representa os deslocamentos (força de superfície) nodais do elemento $\mathrm{j}$.

A aproximação geométrica, feita de maneira análoga pode ser representada da seguinte forma:

$$
\left\{\begin{array}{l}
\mathrm{x} \\
\mathrm{y}
\end{array}\right\}=\left[\begin{array}{cccc}
\phi_{1} & 0 & \phi_{2} & 0 \\
0 & \phi_{1} & 0 & \phi_{2}
\end{array}\right]\left\{\begin{array}{l}
\mathrm{x}^{1} \\
\mathrm{y}^{1} \\
\mathrm{x}^{2} \\
\mathrm{y}^{2}
\end{array}\right\}
$$

Substituindo-se as equações (4.2) e (4.3) em (4.1) tem-se:

$$
[\mathrm{c}][\Phi]^{\mathrm{p}}\{\mathrm{u}\}_{\mathrm{n}}^{\mathrm{p}}+\sum_{\mathrm{j}=1}^{\mathrm{ne}}\left(\int_{\Gamma_{\mathrm{j}}}\left[\mathrm{p}^{*}\right][\Phi] \mathrm{d} \Gamma_{\mathrm{j}}\right)\{\mathrm{u}\}_{\mathrm{n}}^{\mathrm{j}}=\sum_{\mathrm{j}=1}^{\mathrm{ne}}\left(\int_{\Gamma_{\mathrm{j}}}\left[\mathrm{u}^{*}\right][\Phi] \mathrm{d} \Gamma_{\mathrm{j}}\right)\{\mathrm{p}\}_{\mathrm{n}}^{\mathrm{j}}
$$

As integrais da equação (4.5) relacionam os deslocamentos do ponto de colocação, a força de superfície e deslocamentos nodais em qualquer elemento j. Por isso, são denominadas matrizes de influência e seus valores resultantes podem ser representados por

$$
\begin{aligned}
& {[\mathrm{HW}]^{\mathrm{pj}}=\int_{\Gamma_{\mathrm{j}}}\left[\mathrm{p}^{*}\right][\Phi] \mathrm{d} \Gamma_{\mathrm{j}}} \\
& {[\mathrm{GW}]^{\mathrm{pj}}=\int_{\Gamma_{\mathrm{j}}}\left[\mathrm{u}^{*}\right][\Phi] \mathrm{d} \Gamma_{\mathrm{j}}}
\end{aligned}
$$




\subsubsection{Montagem do sistema de Equações}

Substituindo-se as equações (4.6) e (4.7) na eq. (4.5) e considerando-se que:

$$
[\mathrm{HW}]=\left\{\begin{array}{cl}
{[\mathrm{HW}]^{\mathrm{pj}}} & \text { se j } \not \subset \Gamma \\
{[\mathrm{HW}]^{\mathrm{pj}}+[\mathrm{c}][\Phi]^{\mathrm{p}}} & \text { se j } \subset \Gamma
\end{array}\right.
$$

Pode-se chegar a:

$$
\sum_{j=1}^{n e}[H W]^{p j}\{u\}_{n}^{j}=\sum_{j=1}^{n e}[G W]^{p j}\{p\}_{n}^{j}
$$

No problema elástico bidimensional, o número de graus de liberdade é de quatro vezes o número de nós do contorno. Destes, metade são obtidos através das condições de contorno. São necessárias, portanto $2 *$ nn equações, onde nn é o número de nós, para a resolução do problema. Escrevendo-se equações para nn posição do ponto fonte, obtém $2 * n n$ equações, montando-se assim um sistema de equações lineares de ordem $2 * \mathrm{nn}$, cuja resolução fornece as incógnitas restantes:

$$
[\mathrm{H}]\{\mathrm{U}\}=[\mathrm{G}]\{\mathrm{P}\}
$$

\subsubsection{Propriedades da Matriz $H$}

A Matriz $[\mathrm{H}]$ possui uma propriedade bastante útil decorrente do movimento de corpo rígido. Caso um corpo finito se desloque no espaço, sem se deformar, as forças resultantes deverão ser nulas. Logo:

$$
[\mathrm{H}]\{\mathrm{I}\}=\{0\}
$$

Sendo $\{\mathrm{I}\}$ um vetor com deslocamentos de corpo rígido para todos os nós. 
Decorre de (4.11) que, dada uma linha da matriz $[\mathrm{H}]$, a soma das constantes pertencentes a colunas pares será nula. O mesmo acontece com a soma das colunas ímpares. Matematicamente, tem-se:

$$
\sum_{\mathrm{k}=1}^{\mathrm{NN}} \mathrm{H}_{2 \mathrm{k}-1}=0 \quad \text { e } \sum_{\mathrm{k}=1}^{\mathrm{NN}} \mathrm{H}_{2 \mathrm{k}}=0
$$

Caso se trate de um corpo infinito, a integral do núcleo $\mathrm{p}^{*}$, sobre um contorno localizado no infinito, produzirá como resultado as forças do problema de Kelvin, ou seja, uma carga unitária na direção considerada. Logo os somatórios tornam-se iguais a:

$$
\sum_{\mathrm{k}=1}^{\mathrm{NN}} \mathrm{H}_{2 \mathrm{k}-1}=1 \quad \text { e } \sum_{\mathrm{k}=1}^{\mathrm{NN}} \mathrm{H}_{2 \mathrm{k}}=0
$$

para linhas impares, e igual a:

$$
\sum_{\mathrm{k}=1}^{\mathrm{NN}} \mathrm{H}_{2 \mathrm{k}-1}=0 \quad \text { e } \sum_{\mathrm{k}=1}^{\mathrm{NN}} \mathrm{H}_{2 \mathrm{k}}=1
$$

para linhas pares.

\subsubsection{Ponto de colocação}

Para montagem do sistema (4.10), teoricamente, quaisquer conjuntos de pontos de colocação serviriam. Contudo, é conhecido que quando estes pontos se distanciam muito do contorno para fora do domínio, as respostas incorrem num erro considerável.

Se a integração nos elementos for feita de forma numérica estando o ponto fonte muito próximo do contorno, a resposta também não será boa. Para evitar este tipo de problema (no caso em que o ponto está próximo do contorno) a técnica de sub-elementação minimiza o erro de tal integração.

Outro posicionamento do ponto fonte muito interessante que acaba se tornando o mais indicado é aquele em que se coloca o ponto fonte sobre o contorno, introduzindo-se assim um problema a mais na resolução das integrais singulares, que se trata do valor principal de Cauchy, mas se estas integrais forem resolvidas de forma analítica, tem-se um Formulação do Método dos Elementos de Contorno para Análise de Chapas com Enrijecedores. $\quad$ Wilson Wesley Wutzow 
pouco de dificuldade de deduzir as integrais contidas na equação Somigliana, mas o resultado é melhor do que os obtidos pela integração numérica, justificando assim o trabalho necessário para tais deduções.

Neste estudo, varias possibilidades são possíveis, dentre elas podemos citar:

- Ponto fonte fora do contorno com integração analítica.

- Ponto fonte fora do contorno com integração numérica (com sub elementação).

- Ponto fonte no contorno com integração totalmente analítica (tanto a singular como a não-singular).

- Ponto fonte no contorno com integração semi-analítica (sendo a singular analítica e a não-singular numérica com sub-elementação).

Este programa ainda possibilita o posicionamento do ponto fonte quando fora do contorno, tanto na bissetriz como a uma determinada distância perpendicular ao elemento como é mostrado na figura 06.
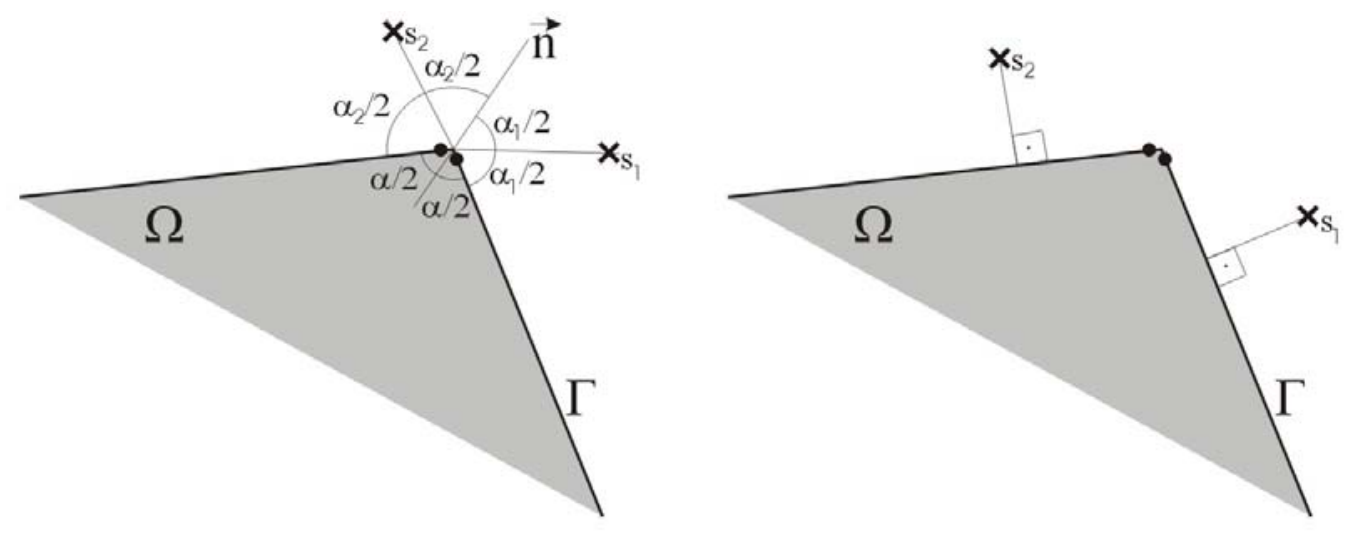

Figura 06 - Posicionamento do ponto fonte.

\subsubsection{Condições de Contorno}

As condições de contorno do problema podem ser descritas das seguintes formas: deslocamentos prescritos, forças de superfície prescritas, ou até mesmo uma relação entre as forças de superfície e deslocamento (como acontece, por exemplo, no caso de um apoio de mola ou inclinado) (apêndice B).

Para suprir a necessidade de condições de contorno a serem prescritas, faz-se necessário à prescrição de duas condições de contorno por nó. Para introduzir as condições 
de contorno em (4.10), as matrizes $[\mathrm{H}]$ e $[\mathrm{G}]$ devem ser rearranjadas de forma que todas as variáveis conhecidas estejam no segundo membro e todas as incógnitas, no primeiro. Isto é feito trocando-se as respectivas colunas de $[\mathrm{H}]$ e $[\mathrm{G}]$, obtendo-se assim o sistema:

$$
[\mathrm{A}]\{\mathrm{VC}\}=[\mathrm{B}]\{\mathrm{VP}\}
$$

Onde $[\mathrm{A}]$ e $[\mathrm{B}]$ são respectivamente, as matrizes modificadas de $[\mathrm{H}]$ e $[\mathrm{G}]$. O vetor $\{\mathrm{VP}\}$ trata-se do vetor que contem os valores prescritos do contorno e $\{\mathrm{VC}\}$ o vetor dos valores de contorno a serem calculados.

\subsubsection{Resolução do sistema de equações}

Pode-se multiplicar o vetor de valores prescritos $\{\mathrm{VP}\}$ pela matriz [B] obtendo-se o sistema linear de equações:

$$
[\mathrm{A}]\{\mathrm{VC}\}=\{\mathrm{C}\}
$$

Onde

$$
\{\mathrm{C}\}=[\mathrm{B}]\{\mathrm{VP}\}
$$

Com o sistema linear apresentado em (4.17) a resolução do sistema é simples de ser obtida, é importante lembrar que a matriz [A] é uma matriz cheia com valores combinados das matrizes $[\mathrm{H}]$ e $[\mathrm{G}]$, sendo os termos $[\mathrm{G}]$ de valores com ordens de grandeza diferentes de $[\mathrm{H}]$ o que acarreta em problemas de condicionamento da matriz [A] e que no caso do emprego de sub-regiões que será descrito mais adiante, esta matriz é esparsa. Para tanto alguns cuidados devem ser tomados na hora de se escolher a rotina a ser empregada na resolução de tal sistema. No programa desenvolvido, empregou-se a rotina de Gauss-Jordan com pivoteamento completo. 
O outro aspecto importante a ser enfatizado é que o vetor de valores calculados $\{\mathrm{VC}\}$ é misto contendo parte de valores de força e parte de valores de deslocamento, o que exige um rearranjo na hora de se visualizar tais valores separando-os de forma coerente.

\subsubsection{Cálculos de deslocamentos, tensões, deformações e forças de superfície.}

Após a resolução do sistema de equações, os valores das forças de contorno e deslocamentos são conhecidos. Logo, determinar os parâmetros adicionais necessários à análise do poblema é um procedimento simples.

\section{Deslocamento em pontos internos}

Os deslocamentos de pontos internos ao domínio podem ser facilmente calculados com auxílio da equação Somigliana que pode ser escrita de maneira sucinta para ponto interno da seguinte forma:

$$
\{u\}^{p}=-\sum_{j=1}^{n e}[H \hat{W}]^{p j}\{u\}_{n}^{j}+\sum_{j=1}^{n e}[G W]^{p j}\{p\}_{n}^{j}
$$

Onde $\{u\}^{\text {p }}$ representa os deslocamentos em um ponto interno 'p' qualquer, portanto através da eq. (4.18) pode-se obter os deslocamentos no ponto 'p' em função dos deslocamentos e forças de superfície nodais.

\section{Deslocamento em pontos sobre os elementos de contorno}

O cálculo dos deslocamentos em pontos situados sob os elementos do contorno (diferente dos nós já calculados na resolução do sistema de equações) neste trabalho é feitos de forma similar ao cálculo dos deslocamentos de pontos internos.

Mas agora usa-se também os termos singulares de integração e deve-se levar em conta a constante que para contorno suave vale $1 / 2$ que já foi comentada no capítulo anterior. 


\section{Tensões em pontos internos}

As tensões em pontos internos podem ser calculadas através das equações constitutivas eq. (2.6), modificadas com aplicação da relação deformação deslocamento eq.(2.4) que esta descrita a seguir:

$$
\sigma_{\mathrm{ij}}^{\mathrm{k}}=\frac{2 \mathrm{G} v}{(1-2 v)} \delta_{\mathrm{ij}} \mathrm{u}_{1,1}+\mathrm{G}\left(\mathrm{u}_{\mathrm{i}, \mathrm{j}}+\mathrm{u}_{\mathrm{j}, \mathrm{i}}\right)
$$

Substituindo-se os termos de deslocamentos da equação acima pela equação Somigliana sem o termo de domínio, logo chega-se a expressão abaixo:

$$
\begin{aligned}
\sigma_{\mathrm{ij}}^{\mathrm{k}}= & \int_{\Gamma}\left\{\frac{2 \mathrm{G} v}{(1-2 v)} \delta_{\mathrm{ij}} \frac{\partial \mathrm{u}_{\mathrm{kk}}^{*}}{\partial \mathrm{x}_{1}}+\mathrm{G}\left(\frac{\partial \mathrm{u}_{\mathrm{ik}}^{*}}{\partial \mathrm{x}_{\mathrm{j}}}+\frac{\partial \mathrm{u}_{\mathrm{jk}}^{*}}{\partial \mathrm{x}_{\mathrm{i}}}\right)\right\} \mathrm{p}_{\mathrm{k}} \mathrm{d} \Gamma+ \\
& \int_{\Gamma}\left\{\frac{2 \mathrm{G} v}{(1-2 v)} \delta_{\mathrm{ij}} \frac{\partial \mathrm{p}_{\mathrm{lk}}^{*}}{\partial \mathrm{x}_{1}}+\mathrm{G}\left(\frac{\partial \mathrm{p}_{\mathrm{ik}}^{*}}{\partial \mathrm{x}_{\mathrm{j}}}+\frac{\partial \mathrm{p}_{\mathrm{jk}}^{*}}{\partial \mathrm{x}_{\mathrm{i}}}\right)\right\} \mathrm{u}_{\mathrm{k}} \mathrm{d} \Gamma
\end{aligned}
$$

$\mathrm{Na}$ forma matricial, tem-se:

$$
\{\sigma\}^{p}=\left\{\begin{array}{l}
\sigma_{11} \\
\sigma_{12} \\
\sigma_{22}
\end{array}\right\}^{p}=\sum_{j=1}^{n e}[D]^{i j k}\{p\}_{n}^{k}-\sum_{j=1}^{n e}[S]^{\mathrm{ijk}}\{u\}_{n}^{k}
$$

Onde os termos $[\mathrm{D}]^{\mathrm{jik}}$ e $[\mathrm{S}]^{\mathrm{ijk}}$ são respectivamente dados por:

$$
[D]^{\mathrm{j} k}=\left[\begin{array}{ll}
\mathrm{D}_{111} & \mathrm{D}_{211} \\
\mathrm{D}_{112} & \mathrm{D}_{212} \\
\mathrm{D}_{122} & \mathrm{D}_{222}
\end{array}\right]=\int_{\Gamma} \frac{1}{4 \pi(1-v) \mathrm{r}}\left\{(1-2 v)\left[\mathrm{r}_{, \mathrm{k}} \delta_{\mathrm{ij}}+\mathrm{r}_{\mathrm{j}} \delta_{\mathrm{ik}}-\mathrm{r}_{\mathrm{i}} \delta_{\mathrm{jk}}\right]+2 \mathrm{r}_{, \mathrm{i}} \mathrm{r}_{\mathrm{j}, \mathrm{r}} \mathrm{r}_{\mathrm{k}}\right\} \mathrm{d} \Gamma
$$




$$
\begin{aligned}
& {[S]^{\mathrm{jik}}=\left[\begin{array}{ll}
\mathrm{S}_{111} & \mathrm{~S}_{211} \\
\mathrm{~S}_{112} & \mathrm{~S}_{212} \\
\mathrm{~S}_{122} & \mathrm{~S}_{222}
\end{array}\right]=\int_{\Gamma} \frac{\mathrm{G}}{2 \pi(1-v) \mathrm{r}^{2}}\left\{2 \mathrm { r } _ { , \mathrm { n } } \left[(1-2 v) \delta_{\mathrm{jk}} \mathrm{r}_{, \mathrm{i}}+v\left(\delta_{\mathrm{ji}} \mathrm{r}_{\mathrm{k}}+\delta_{\mathrm{ik}} \mathrm{r}_{, \mathrm{j}}\right)+\right.\right.} \\
& \left.-4 \mathrm{r}_{, \mathrm{i}, \mathrm{j}, \mathrm{r}} \mathrm{r}_{, \mathrm{k}}\right]+2 v\left[\mathrm{n}_{\mathrm{j}, \mathrm{r}} \mathrm{r}_{, \mathrm{i}}+\mathrm{n}_{\mathrm{k}, \mathrm{j}, \mathrm{r}} \mathrm{r}_{\mathrm{i}}\right]+(1-2 v)\left[\mathrm{n}_{\mathrm{i}, \mathrm{r}, \mathrm{r}} \mathrm{r}_{\mathrm{k}}+\mathrm{n}_{\mathrm{k}} \delta_{\mathrm{ji}}+\mathrm{n}_{\mathrm{j}} \delta_{\mathrm{ki}}\right] \\
& \left.-(1-4 v) \mathrm{n}_{\mathrm{i}} \delta_{\mathrm{jk}}\right\} \mathrm{d} \Gamma
\end{aligned}
$$

Onde $r_{, n}$ indica a derivada do raio (módulo do vetor posição) na direção do versor normal ao elemento.

\section{Tensões em pontos sobre os elementos de contorno}

O cálculo das tensões em pontos situados sob os elementos de contorno (diferente dos nós já calculados na resolução do sistema de equações) neste trabalho, assim como para o caso dos deslocamentos, são feitos de forma similar ao cálculo das tensões de pontos internos só que agora se deve empregar ainda as soluções para as integrais singulares, quando o ponto fonte estiver sobre o elemento em que se está integrando. As deduções de tais expressões serão comentadas mais à frente e as expressões finais são apresentadas nos anexos D e E.

\section{Tensões nos nós do contorno}

Este item apesar de saber-se que há um problema de indeterminação dos valores de tensão para as extremidades dos elementos de contorno, para efeito de pós-processamento, mapas de tensão, e isolinhas de tensão são necessários que algum valor de tensão sejam atribuído ás extremidades dos elementos, sabendo-se, contudo que isto implicaria num erro considerável. Contudo para análises e estudos dos resultados dos exemplos simulados, as tensões que são empregadas são aquelas encontradas em pontos internos ou em pontos localizados no contorno não estando estes sob os nós do contorno.

O cálculo de tensões sob os nós do contorno foi feito calculando-se a tensão em dois pontos situados nos elementos de contorno ( $2 / 3$ e 1/3 do comprimento do elemento) como é apresentado na figura 07 , de posse destes valores é feita uma extrapolação para os valores de tensão das extremidades dos elementos. E por fim é feita uma média entre a tensão de extremidade do elemento com a tensão de extremidade do elemento vizinho. 


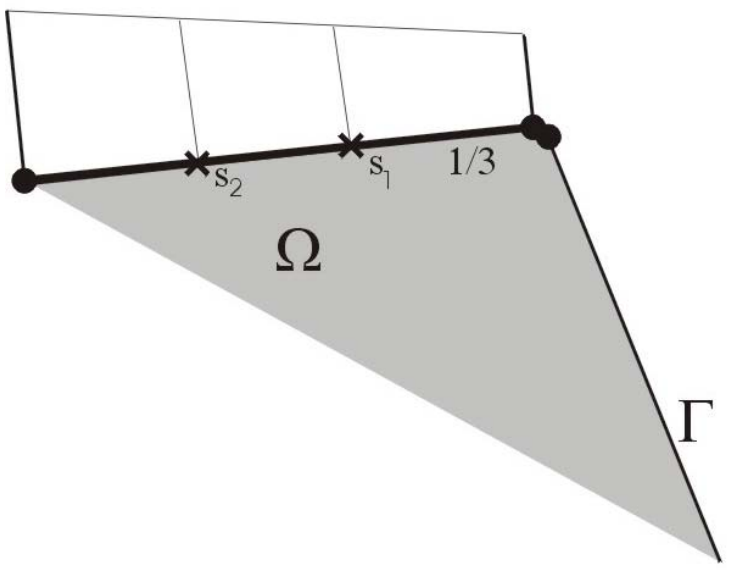

Figura 07 - Extrapolação da tensão interna ao elemento para os nós do contorno.

\section{Deformações em pontos internos e do contorno}

Tendo-se o vetor de tensões do ponto em questão, basta aplicar a lei de Hooke para se obter as respectivas deformações do ponto.

\subsection{INTEGRAIS ANALÍTICAS}

Esta seção tem por objetivo descrever os resultados das integrais de forma analítica dos termos das matrizes $\mathrm{H}, \mathrm{G}, \mathrm{S}$ e $\mathrm{D}$, tanto as singulares quanto as não singulares.

Contudo devido à extensão destas equações, será apresentado somente um exemplo de dedução e os resultados serão apresentados nos anexos D e E.

Termos a serem calculados:

$$
\left[H_{\mathrm{z}}\right]=\left[\begin{array}{lll}
\mathrm{H}_{11}^{1} & \mathrm{H}_{12}^{1} \vdots \mathrm{H}_{11}^{2} & \mathrm{H}_{12}^{2} \\
\mathrm{H}_{21}^{1} & \mathrm{H}_{22}^{1} \vdots \mathrm{H}_{21}^{2} & \mathrm{H}_{22}^{2}
\end{array}\right] \quad\left[\mathrm{G}_{\mathrm{z}}\right]=\left[\begin{array}{ccc}
\mathrm{G}_{11}^{1} & \mathrm{G}_{12}^{1} \vdots \mathrm{G}_{11}^{2} & \mathrm{G}_{12}^{2} \\
\mathrm{G}_{21}^{1} & \mathrm{G}_{22}^{1} \vdots \mathrm{G}_{21}^{2} & \mathrm{G}_{22}^{2}
\end{array}\right]
$$




$$
\left[S_{z}\right]=\left[\begin{array}{lll}
S_{11}^{1} & S_{12}^{1} \vdots S_{11}^{2} & S_{12}^{2} \\
S_{21}^{1} & S_{22}^{1} \vdots S_{21}^{2} & S_{22}^{2} \\
S_{31}^{1} & S_{32}^{1} \vdots S_{31}^{2} & S_{32}^{2}
\end{array}\right] \quad\left[D_{z}\right]=\left[\begin{array}{lll}
D_{11}^{1} & D_{12}^{1} \vdots D_{11}^{2} & D_{12}^{2} \\
D_{21}^{1} & D_{22}^{1} \vdots D_{21}^{2} & D_{22}^{2} \\
D_{31}^{1} & D_{32}^{1} \vdots D_{31}^{2} & D_{32}^{2}
\end{array}\right]
$$

onde:

$$
\begin{aligned}
& {[\mathrm{H}]_{\mathrm{ij}}^{\mathrm{k}}=\int_{\Gamma} \frac{-1}{4 \pi(1-v) \mathrm{r}}\left\{\left[(1-2 v) \delta_{\mathrm{ij}}+2 \mathrm{r}_{, \mathrm{i}} \mathrm{r}_{\mathrm{j}}\right] \mathrm{r}_{, \mathrm{n}}-(1-2 v)\left(\mathrm{r}_{, \mathrm{i}} \mathrm{n}_{\mathrm{j}}-\mathrm{r}_{, \mathrm{j}} \mathrm{n}_{\mathrm{i}}\right)\right\} \mathrm{d} \Gamma} \\
& {[G]_{i j}^{\mathrm{k}}=\int_{\Gamma} \frac{1}{8 \pi \mathrm{G}(1-v)}\left[(3-4 v) \ln \left(\frac{1}{\mathrm{r}}\right) \delta_{\mathrm{ij}}+\mathrm{r}_{, \mathrm{i}} \mathrm{r}_{\mathrm{j}}\right] \mathrm{d} \Gamma} \\
& {[S]_{\mathrm{ij}}^{\mathrm{k}}=\int_{\Gamma} \frac{\mathrm{G}}{2 \pi(1-v) \mathrm{r}^{2}}\left\{2 \mathrm{r}_{, \mathrm{n}}\left[(1-2 v) \delta_{\mathrm{jk}} \mathrm{r}_{, \mathrm{i}}+v\left(\delta_{\mathrm{ji}} \mathrm{r}_{\mathrm{k}}+\delta_{\mathrm{ik}} \mathrm{r}_{\mathrm{j}}\right)-4 \mathrm{r}_{, \mathrm{i}} \mathrm{r}_{\mathrm{j}, \mathrm{r}} \mathrm{r}_{\mathrm{k}}\right]\right.} \\
& \left.+2 v\left[\mathrm{n}_{\mathrm{j}, \mathrm{r}} \mathrm{r}_{, \mathrm{i}}+\mathrm{n}_{\mathrm{k}, \mathrm{r}, \mathrm{r}} \mathrm{r}_{\mathrm{i}}\right]+(1-2 v)\left[\mathrm{n}_{\mathrm{i}, \mathrm{j}, \mathrm{r}} \mathrm{r}_{\mathrm{k}}+\mathrm{n}_{\mathrm{k}} \delta_{\mathrm{ji}}+\mathrm{n}_{\mathrm{j}} \delta_{\mathrm{ki}}\right]-(1-4 v) \mathrm{n}_{\mathrm{i}} \delta_{\mathrm{jk}}\right\} \mathrm{d} \Gamma \\
& {[D]_{\mathrm{ij}}^{\mathrm{k}}=\int_{\Gamma} \frac{1}{4 \pi(1-v) \mathrm{r}}\left\{(1-2 v)\left[\mathrm{r}_{, \mathrm{k}} \delta_{\mathrm{ij}}+\mathrm{r}_{\mathrm{j} j} \delta_{\mathrm{ik}}-\mathrm{r}_{\mathrm{i}} \delta_{\mathrm{jk}}\right]+2 \mathrm{r}_{, \mathrm{i}} \mathrm{r}_{\mathrm{j}, \mathrm{r}} \mathrm{r}_{\mathrm{k}}\right\} \mathrm{d} \Gamma}
\end{aligned}
$$

Note que para facilitar a dedução de tais integrais, nem sempre foi adotado um mesmo sistema de coordenadas, portanto na hora de implementar tais deduções, estes parâmetros devem ser levados em conta tanto para fornecer corretamente os dados de cálculo dos termos quanto após o cálculo dos termos das matrizes, deve-se fazer as respectivas transformações de forma coerente para o sistema global novamente.

\subsubsection{Integrais não singulares}

Para as integrais não singulares, ponto fonte fora do elemento a ser integrado, dividiu-se em três possibilidades, que são: ponto fonte fora do alinhamento do elemento, ponto fonte alinhado com o elemento atrás do mesmo, ponto fonte alinhado com o elemento e na frente do elemento. 


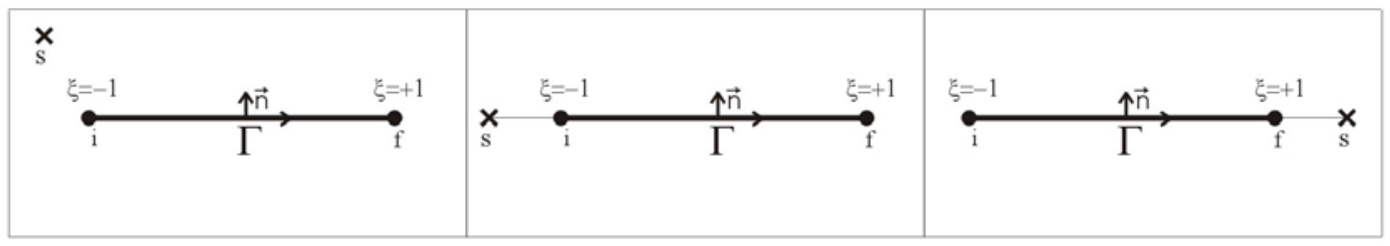

Figura 08 - Casos para integração não singular.

Para ilustrar a dedução de tais integrais será apresentado de forma detalhada a dedução do termo $\mathrm{D}_{11}^{1}$ para ponto fonte fora do elemento a ser integrado (integral não singular) com o ponto fonte não alinhado com o elemento. Como está ilustrado na figura a seguir:

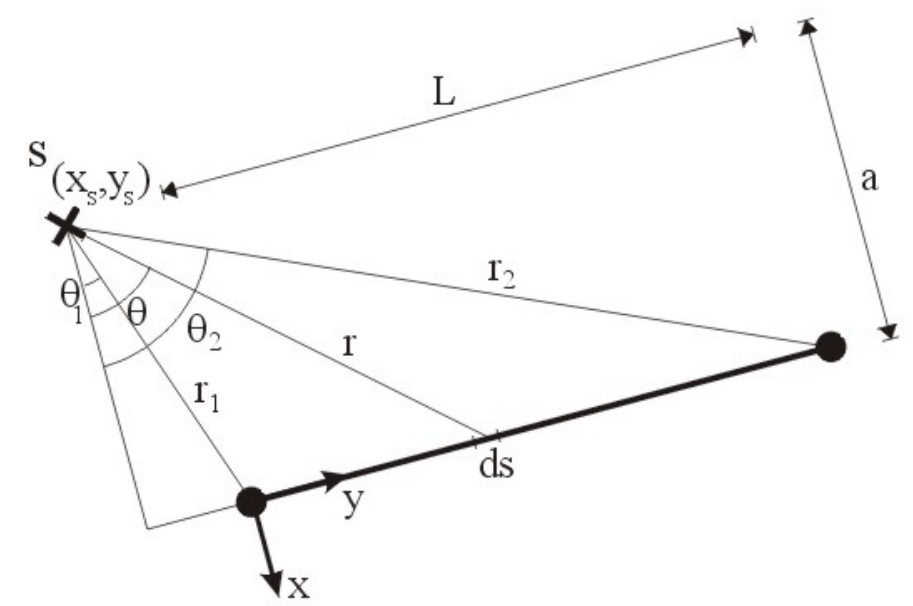

Figura 09 - Transformação de coordenadas para integração analítica não singular com ponto fonte não alinhado com o elemento.

Pela figura 09, pode-se concluir que:

$\operatorname{Cos} \theta=\frac{\mathrm{a}}{\mathrm{r}}$, ou $\mathrm{r}=\frac{\mathrm{a}}{\operatorname{Cos} \theta}$

$\operatorname{Sen} \theta=\frac{\mathrm{y}}{\mathrm{r}}$

$\operatorname{Tg} \theta=\frac{y-y_{s}}{a}$, ou $y=a * \operatorname{tg} \theta+y_{s}$

$\mathrm{ds}=\mathrm{dy}=\mathrm{y}^{\prime} \mathrm{d} \theta=\operatorname{asec}^{2} \theta d \theta=\left(\frac{\mathrm{a}}{\cos ^{2} \theta}\right) \mathrm{d} \theta$ 
$r_{, 1}=\cos \theta \quad$ e $\quad r_{,_{2}}=\operatorname{sen} \theta$

$\mathrm{n}_{1}=\cos (\mathrm{n}, \mathrm{x})=\cos 0^{\circ}=1$ e $\mathrm{n}_{2}=\cos (\mathrm{n}, \mathrm{y})=\cos 90^{\circ}=0$

$\mathrm{r}_{\mathrm{n}}=\frac{\partial \mathrm{r}}{\partial \mathrm{n}}=\frac{\partial \mathrm{r}}{\partial \mathrm{x}} \mathrm{n}_{\mathrm{x}}+\frac{\partial \mathrm{r}}{\partial \mathrm{y}} \mathrm{n}_{\mathrm{y}}=\mathrm{r}_{,_{1}} \mathrm{n}_{1}+\mathrm{r}_{,_{2}} \mathrm{n}_{2}=\cos \theta * 1+\operatorname{sen} \theta * 0=\cos \theta$

$\delta_{\mathrm{ij}}=\left[\begin{array}{ll}1 & 0 \\ 0 & 1\end{array}\right]$

Logo se pode escrever as funções de forma desta maneira:

$\phi_{1}=1-\frac{\mathrm{y}}{\mathrm{L}}=1-\frac{\mathrm{y}_{\mathrm{s}}}{\mathrm{L}}-\mathrm{a} \frac{\operatorname{tg} \theta}{\mathrm{L}}$

$\phi_{2}=\frac{\mathrm{y}}{\mathrm{L}}=\frac{\mathrm{y}_{\mathrm{s}}}{\mathrm{L}}+\mathrm{a} \frac{\operatorname{tg} \theta}{\mathrm{L}}$
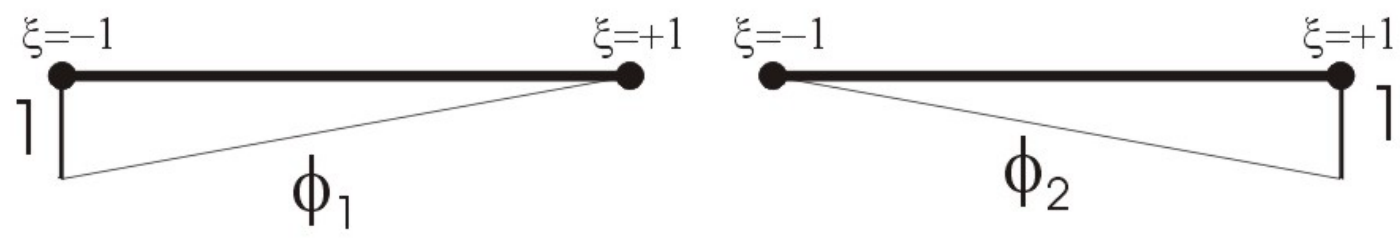

Figura 10 - Funções de forma.

Como já vimos anteriormente:

$[D]_{\mathrm{ij}}^{\mathrm{k}}=\int_{\Gamma} \frac{1}{4 \pi(1-v) \mathrm{r}}\left\{(1-2 v)\left[\mathrm{r}_{, \mathrm{k}} \delta_{\mathrm{ij}}+\mathrm{r}_{\mathrm{j}} \delta_{\mathrm{ik}}-\mathrm{r}_{, \mathrm{i}} \delta_{\mathrm{jk}}\right]+2 \mathrm{r}_{, \mathrm{i}} \mathrm{r}_{\mathrm{j}, \mathrm{r}} \mathrm{r}_{\mathrm{k}}\right\} \mathrm{d} \Gamma$

Para i,j,k,l=1, agrupando algumas constantes da seguinte maneira:

$\mathrm{ek}=\frac{1}{4 \pi(1-v)}$

$\mathrm{ek} 1=(1-2 v)$ 
E levando-se em conta as variáveis definidas anteriormente em função de $\theta$ tem-se:

$$
\begin{aligned}
& \mathrm{D}_{11}^{1}=\int_{\theta_{1}}^{\theta_{2}} \mathrm{ek} \frac{\cos \theta}{\mathrm{a}}\left\{\mathrm{ek}_{1} \cos \theta+2 \cos ^{3} \theta\right\}\left(1-\frac{\mathrm{y}_{\mathrm{s}}}{\mathrm{L}}-\frac{\mathrm{a}}{\mathrm{L}} \operatorname{tg} \theta\right) \frac{\mathrm{a}}{\cos ^{2} \theta} \mathrm{d} \theta \\
& \mathrm{D}_{11}^{1}=\int_{\theta_{1}}^{\theta_{2}} \mathrm{ek}\left\{\mathrm{ek}_{1}+2 \cos ^{2} \theta\right\}\left(1-\frac{\mathrm{y}_{\mathrm{s}}}{\mathrm{L}}-\frac{\mathrm{a}}{\mathrm{L}} \operatorname{tg} \theta\right) \mathrm{d} \theta \\
& \mathrm{D}_{11}^{1}=\mathrm{ek} \int_{\theta_{1}}^{\theta_{2}}\left\{\mathrm{ek}_{1}\left(1-\frac{\mathrm{y}_{\mathrm{s}}}{\mathrm{L}}\right)+2 \cos ^{2} \theta\left(1-\frac{\mathrm{y}_{\mathrm{s}}}{\mathrm{L}}\right)-\mathrm{ek}_{1} \frac{\mathrm{a}}{\mathrm{L}} \operatorname{tg} \theta-2 \frac{\mathrm{a}}{\mathrm{L}} \cos \theta \operatorname{sen} \theta\right\} \mathrm{d} \theta \\
& D_{11}^{1}=\left.e k\left\{\left(e_{1} \theta+\theta+\cos \theta \operatorname{sen} \theta\right)\left(1-\frac{y_{s}}{L}\right)+\left(e_{1} \operatorname{Ln}(\cos \theta)+\cos ^{2} \theta\right) \frac{a}{L}\right\}\right|_{\theta_{1}} ^{\theta_{2}} \\
& \varphi_{1}=\left(1-\frac{\mathrm{y}_{\mathrm{s}}}{\mathrm{L}}\right) \\
& \mathrm{D}_{11}^{1}=\mathrm{ek}\left\{\left[\left(\mathrm{ek}_{1}+1\right) \theta_{2}+\cos \theta_{2} \operatorname{sen} \theta_{2}\right] \varphi_{1}+\left(\mathrm{ek}_{1} \operatorname{Ln}\left(\cos \theta_{2}\right)+\cos ^{2} \theta_{2}\right) \frac{\mathrm{a}}{\mathrm{L}}\right\} \\
& -\mathrm{ek}\left\{\left[\left(\mathrm{ek}_{1}+1\right) \theta_{1}+\cos \theta_{1} \operatorname{sen} \theta_{1}\right] \varphi_{1}+\left(\mathrm{ek}_{1} \operatorname{Ln}\left(\cos \theta_{1}\right)+\cos ^{2} \theta_{1}\right) \frac{\mathrm{a}}{\mathrm{L}}\right\}
\end{aligned}
$$

De forma semelhante, os outros termos das matrizes $H, G, S$ e D são deduzidos, e os resultados estão apresentados no anexo D.

\subsubsection{Integrais singulares}

Quando o ponto fonte pertence ao elemento de integração, o integrando no cálculo das matrizes de influência apresentará singularidade devido à solução fundamental. Para este caso, neste trabalho, o cálculo singular das matrizes de influência foi feito analiticamente e os coeficientes calculados são listados a seguir. Dando a atenção necessária ao valor principal de Cauchy quando era o caso de uma integral de $1 / \mathrm{r}$, e ao valor principal de Hadamard quando era o caso de uma integral de $1 / \mathrm{r}^{2}$. 
Três casos foram considerados, dependendo da posição do ponto fonte ao longo do elemento de integração: No início, em alguma posição intermediária, ou no final. Tanto o primeiro quanto o terceiro caso podem ser considerados como particularização do segundo caso.

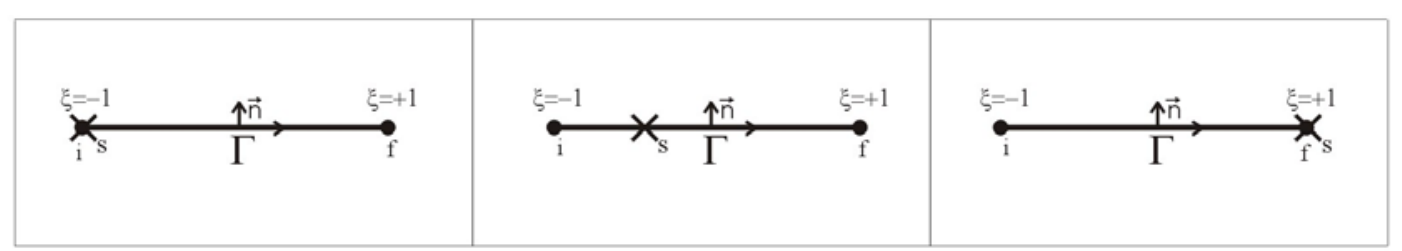

Figura 11 - Casos para integração singular.

As equações já integradas são apresentadas no anexo E.

No primeiro e no terceiro caso, a parte singular das integrais de $\mathrm{H}$ e G sempre irão se anular se considerarmos a outra parte singular do elemento adjacente ao mesmo.

Como foi citado anteriormente, no caso das integrais de S e D para cálculo de tensão, somente o segundo caso (ponto interno ao elemento) foi calculado, isto por causa da indeterminação existente para tais integrais em contornos não suaves.

\subsection{INTEGRAÇÃo NUMÉRICA}

No caso da integração não singular, desenvolveu-se no programa tanto a opção de integração totalmente analítica, ou a parcialmente analítica, sendo neste segundo caso as integrais não singulares calculadas de forma numérica através da quadratura de GaussLegendre.

De maneira geral a integração numérica é empregada quando o cálculo analítico de integrais da forma

$\int_{a}^{b} F(x) d x$

apresenta alguma dificuldade para ser obtida de forma exata devido à complexidade do integrando F. A idéia básica é a de que uma integral com limites arbitrários pode ser 
transformada através da utilização de coordenadas naturais e, então calculada numericamente.

Em vários problemas onde se requer integração numérica, é necessário efetuar a transformação da coordenada global do problema (por exemplo, x) para uma coordenada local $\xi$, de forma que, quando $\mathrm{x}=\mathrm{x}_{\mathrm{A}}, \xi=-1$ e, quando $\mathrm{x}=\mathrm{x}_{\mathrm{B}}, \xi=1$. A coordenada local $\xi$ é também conhecida por coordenada normal, natural ou homogênea.

\subsubsection{Quadratura de Gauss-Legendre}

Quadratura é o termo empregado para o cálculo numérico de integrais (em lugar de cálculo analítico através de tabelas). Existem várias regras de quadratura: Newton-Cotes, Gauss-Legendre, Gaussiana Logarítmica e etc.

A quadratura gaussiana (Gauss-Legendre) consiste em se aproximar o valor de uma dada integral, em um intervalo normalizado de -1 a 1 , pela integral de um polinômio interpolador da função neste trecho. A integral deste polinômio é calculada fazendo-se o somatório do valor da função polinomial em determinadas abscissas, multiplicadas por fatores de ponderação, conhecidos como pesos.

A fórmula de integração de Gauss-Legendre é dada por

$$
\int_{a}^{b} F(x) d x=\int_{-1}^{1} F(x(\xi))|j| d \xi \approx \sum_{i=1}^{n} F(\xi)|j|_{i} \omega_{i}
$$

Onde

$\omega_{\mathrm{i}}$ são os fatores de ponderação;

$\xi_{\mathrm{i}}$ são os pontos-base;

$|j|_{i}$ indica o jacobiano da transformação de coordenadas, calculado no i-ésimo ponto de Gauss. 
Para o elemento isoparamétrico linear, o jacobiano assume um valor constante igual à metade do comprimento do elemento. Para elementos de grau mais elevado este valor deve ser calculado ponto a ponto segundo a seguinte equação:

$J(\xi)=\frac{d \Gamma}{d \xi}=\sqrt{\left(\frac{d x(\xi)}{d \xi}\right)^{2}+\left(\frac{d y(\xi)}{d \xi}\right)^{2}}$

Sendo:

$\frac{d x(\xi)}{d \xi}=\frac{d \phi_{i}(\xi)}{d \xi} x_{i} \quad$ e $\frac{d y(\xi)}{d \xi}=\frac{d \phi_{i}(\xi)}{d \xi} y_{i}$

Com i variando de 1 até $\mathrm{n}+1$.

\subsubsection{Sub-elementação}

A integração numérica conduz a bons resultados quando o ponto de colocação não está muito próximo do elemento a ser integrado, contudo, caso isso ocorra, haverá um problema de quase singularidade, ou seja, os núcleos das integrais passam a ter gradiente muito elevado e fazem a integral numérica divergir.

Para melhorar os resultados obtidos através da integração numérica para pontos fontes muito próximos aos elementos de contorno pode-se introduzir técnicas que melhoram estas integrações.

Uma maneira de se evitar este problema de quase singularidade é utilizar-se de subelementos, que consiste basicamente em dividir o elemento de integração em sub-elementos menores (de comprimento padronizado ou progressivo). Os sub-elementos padronizados, de tamanhos iguais, não são tão eficientes quanto aos sub-elementos progressivos, de tamanho variável, cujo tamanho é função da distância do ponto de colocação.

Considera-se que seja válida a relação:

$\int_{\Gamma_{j}} f\left(\Gamma_{j}\right) \phi_{\eta} d \Gamma_{j}=\sum_{i=1}^{N \text { Sub elem }} f f\left(\Gamma_{j i}\right) \phi_{\eta} d \Gamma_{j i}$ 


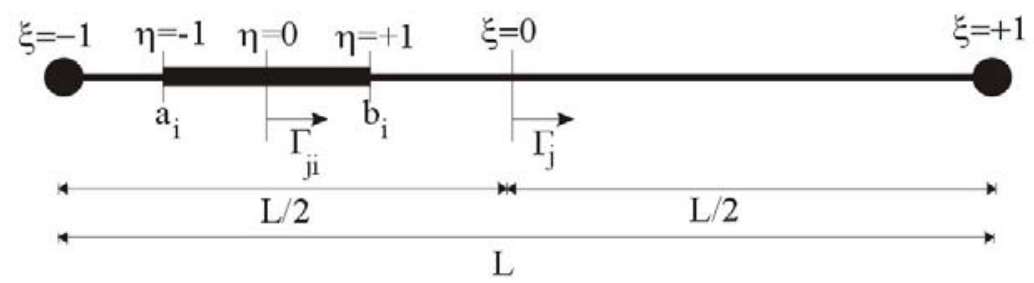

Figura 12 - Coordenadas adimensionais $\eta$ e $\xi$.

Transformando-se para as coordenadas adimensionais $\eta$, isto é, para:

$\Gamma=\mathrm{a}_{\mathrm{i}} \rightarrow \eta=-1$

$\Gamma=b_{\mathrm{i}} \rightarrow \eta=1$

Logo:

$\int_{\Gamma_{j}} f\left(\Gamma_{j}\right) \phi_{\eta} d \Gamma_{j}=\sum_{i=1}^{N \text { Sub elem }} \frac{I_{i}}{2} \int_{-1}^{1} f(\eta) \phi_{\eta} d \eta$

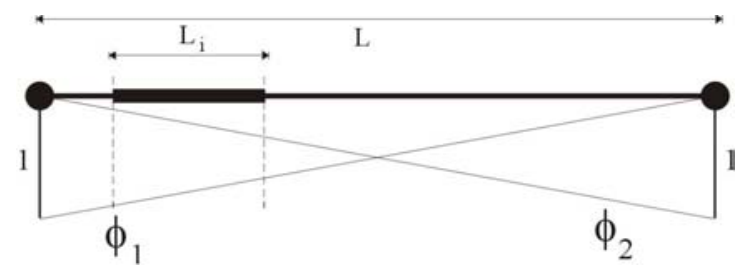

Figura 13 - Funções de forma.

Pode-se observar que $\phi_{\eta}(\xi)$ esta relacionado com a coordenada adimensional $\xi$, pois $\phi_{\eta}(\xi)$ são funções de forma, isto é, relaciona o valor de uma dada variável em função dos valores nodais do elemento figura13.

Pelo fato do intervalo de integração estar em função da coordenada adimensional $\eta[-1 ;+1]$ e sendo a função de forma expressa em coordenada adimensional $\xi$, há necessidade de se fazer a correlação entre as duas coordenadas adimensionais.

Deve-se fazer uma pesquisa para identificar se o elemento será dividido em subelementos ou não. Para identificar se o elemento precisa ou não ser dividido em subelementos e qual o tamanho deste, considere-se a figura 14 , onde se tem um elemento que será integrado numericamente, sendo o ponto $\mathrm{S}$, próximo ao elemento.

Inicialmente calcula-se à distância (rs) do ponto de colocação ao início do elemento, dada por: 
$r s=\sqrt{\left(x_{i}-x_{a}\right)^{2}+\left(y_{i}-y_{a}\right)^{2}}$

Calcula-se também o seno e o co-seno do ângulo formado por esta reta (rs), isto é:

$\cos \beta=\frac{x_{a}-x_{i}}{r s}$
$\operatorname{sen} \beta=\frac{y_{a}-y_{i}}{r s}$

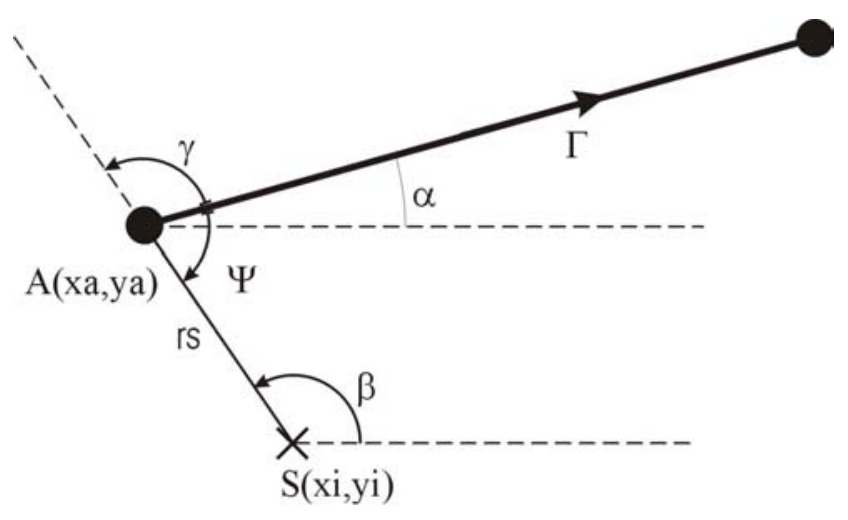

Figura 14 - Determinação dos ângulos para o sub-elemento.

Pela figura 14, pode-se concluir que

$\gamma=\beta-\alpha \quad \Rightarrow \quad \cos (\gamma)=\cos (\beta-\alpha)=\cos (\alpha) \cos (\beta)+\operatorname{sen}(\alpha) \operatorname{sen}(\beta)$

Pode-se obter o ângulo $\Psi$, fazendo-se para isso:

$\Psi=\pi-\gamma$

Se o ângulo $\Psi$ for menor que $60^{\circ}$ então o comprimento do sub-elemento será dado pela interseção da mediatriz da distância rs com o elemento de integração, figura 15. 


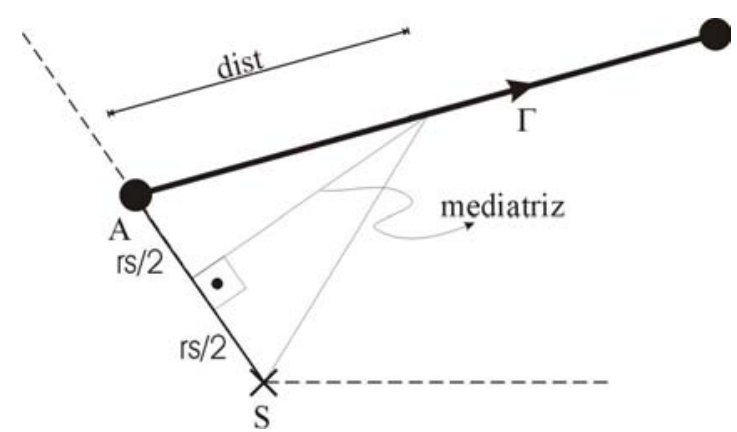

Figura 15 - Determinação dp comprimento para o sub-elemento.

Com isso o comprimento do sub-elemento (dist), será dado pela relação:

$$
\cos (\Psi)=\frac{(\mathrm{rs} / 2)}{\operatorname{dist}} \Rightarrow \operatorname{dist}=\left|\frac{\mathrm{rs}}{2 \cos (\gamma)}\right|
$$

\section{Como $\Psi=\pi-\gamma$}

Logo: $\cos (\Psi)=-\cos (\gamma)$

Quando o ângulo $\Psi$ for maior que $60^{\circ}$ então o comprimento do sub-elemento (dist) será igual ao comprimento da distância rs, figura 16.

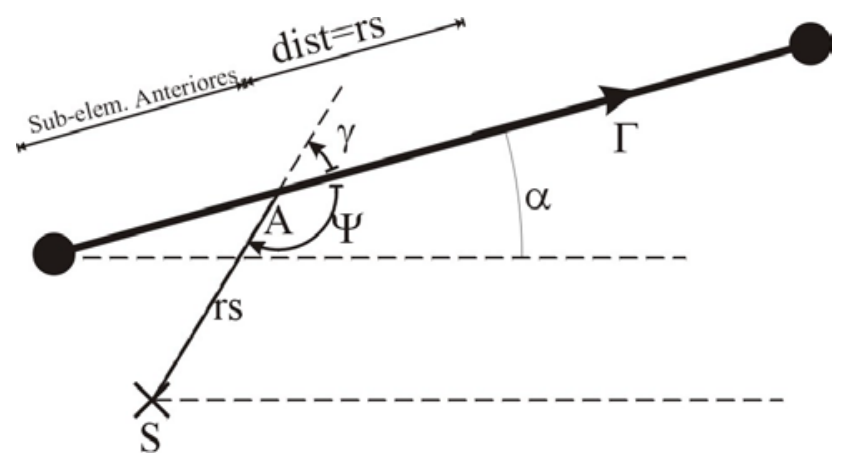

Figura 16 - Comprimento do sub-elemento para $\psi$ maior que $60^{\circ}$.

Repete-se este processo até o somatório dos sub-elementos ser maior ou igual ao elemento de integração. Caso o sub-elemento seja maior, então este último sub-elemento terá 
comprimento igual ao que resta para completar o somatório, o que acontece com r8 na figura 17.

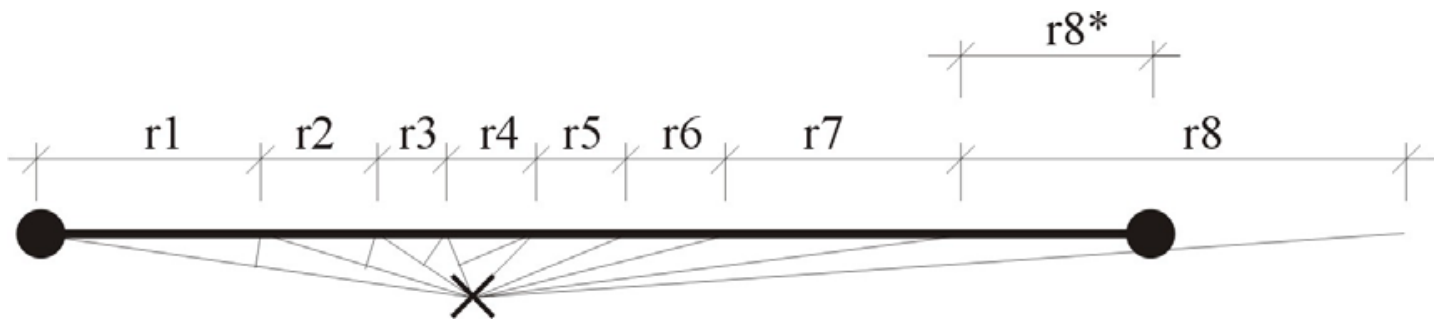

Figura 17 - Divisão do elemento em sub-elementos. 


\section{Sistema LineAR SuAVE - Mínimos QuAdRAdos}

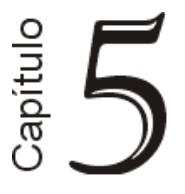

O método dos elementos de contorno pode apresentar em certas situações oscilações dos resultados. Isso ocorre com certas condições de contorno (descontinuidades) e principalmente ao longo de interfaces de sub-regiões com grandes diferenças de módulo de elasticidade ou em acoplamento MEC/MEF. Nestes casos, os resultados satisfazem o equilíbrio, porém com grandes oscilações. Para corrigir essa distorção do método, sem que seja necessário para isto o aumento excessivo da discretização, uma das alternativas é escrever um número maior de equações algébricas para o mesmo problema e obter a solução com o método dos mínimos quadrados.

Sobre a técnica de suavização, dois tipos de melhorias de resultados podem ser esperados, o primeiro já descrito acima diz respeito à minimização das oscilações, o segundo se refere ao fato de que além de minimizar as oscilações pode-se chegar a resultados que represente melhor o problema analisado, a partir de um sistema de equações menor do que o que normalmente seria necessário, já que o sistema de equações após a aplicação da técnica deve representar melhor o problema físico do que o sistema de equações original, não exigindo assim um aumento da discretização.

Esta técnica pode ser empregada tanto por sub-região como também por elemento, sendo a segunda muito mais rápida que a primeira. Estas duas maneiras de se aplicar à técnica são apresentadas a seguir.

Como apresentar os conceitos básicos do método dos mínimos quadrados usando para isso o método dos elementos de contorno seria algo que imporia um determinado grau de dificuldade levando talvez a incompreensão do método optou-se por fazem uma explanação dos conceitos básicos em um problema mais simples, explanação esta apresentada no item 5.1. Já a aplicação da técnica de mínimos quadrados ao método dos Formulação do Método dos Elementos de Contorno para Análise de Chapas com Enrijecedores. Wilson Wesley Wutzow 
elementos de contorno é demonstrado de maneira bastante sucinta nos itens 5.2 (mínimos quadrados por sub-região) e 5.3 (mínimos quadrados por elemento). E finalmente no item 5.4 são apresentados alguns exemplos de aplicação da técnica.

\subsection{CONCEITUAÇÃo BÁSICA SOBRE O MÉTODO DOS MÍNIMOS QUADRADOS}

Ao aproximar uma função $f$ por uma função $g$ de uma família $G$ estaremos introduzindo um erro r que será chamado de resíduo.

Assim

$$
r(x)=f(x)-g(x)
$$

Aparentemente, uma "boa" aproximação seria obtida fazendo $\sum_{\mathrm{x}} \mathrm{r}(\mathrm{x})=0$. Analisemos tal afirmação, suponha que foi realizado um experimento em que se levantou os pontos p1, p2, p3 e p4. Sabendo-se que o fenômeno é descrito por uma reta, vamos determina-la de modo a satisfazer $\sum_{\mathrm{x}} \mathrm{r}(\mathrm{x})=0$. Pode-se observar na figura 18 que todas as retas que foram traçadas obedecem tal critério, o que mostra que $\sum_{\mathrm{x}} \mathrm{r}(\mathrm{x})=0$ não é uma boa escolha.

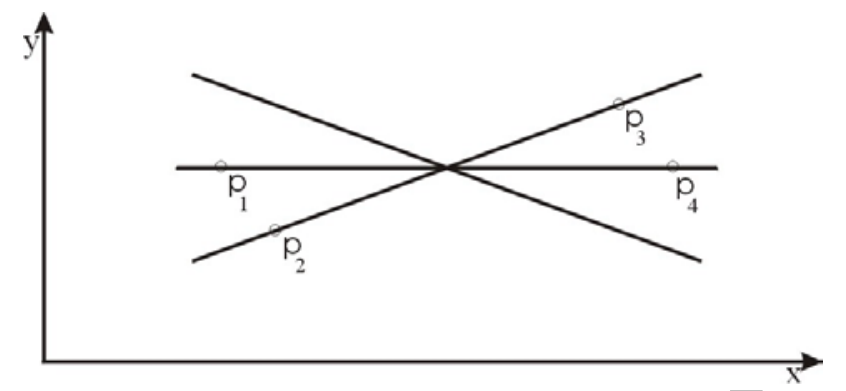

Figura 18 - Aproximações possíveis considerando $\sum_{\mathrm{x}} \mathrm{r}(\mathrm{x})=0$.

O problema que estamos enfrentando com este critério é o fato dos erros positivos cancelarem os erros negativos. Portanto, se deixarmos de considerar o sinal dos erros, evitaremos este problema. Isso pode ser feito trabalhando com valor absoluto dos resíduos e exigindo que $\sum_{\mathrm{x}}|\mathrm{r}(\mathrm{x})|=0$ seja mínimo. Achar o mínimo desta função nos leva a uma 
dificuldade matemática não desejada. Um outro critério com a mesma característica, porém com tratamento matemático mais simples, é exigir que $\sum_{\mathrm{x}} \mathrm{r}^{2}(\mathrm{x})=0$ seja mínimo. O método para aproximar uma função $f$ por uma $g \in \mathrm{G}$ utilizando esse último critério é denominado método dos mínimos quadrados.

De acordo com VOLTERRA (1956), a solução de um sistema de equações $[\mathrm{A}]_{\mathrm{mn}}\{\mathrm{X}\}_{\mathrm{n}}=\{\mathrm{B}\}_{\mathrm{m}}$ onde $\mathrm{m}>\mathrm{n}$ pelo método dos mínimos quadrados é equivalente a resolver o seguinte sistema de equações: $[\mathrm{A}]^{\mathrm{T}}[\mathrm{A}]\{\mathrm{X}\}=[\mathrm{A}]^{\mathrm{T}}\{\mathrm{B}\}$.

\subsection{MÍNIMOS QUADRADOS POR SUB-REGIÃO}

A técnica de suavização do contorno com aplicação dos mínimos quadrados consiste basicamente em gerar mais equações do que as necessárias aumentando o número de pontos fontes. Para reduzir então esta super abundância de equações substitui-se o sistema:

$[\mathrm{H}]\{\mathrm{U}\}=[\mathrm{G}]\{\mathrm{P}\}$

para o sistema

$\left[\mathrm{H}^{*}\right]^{\mathrm{T}}\left[\mathrm{H}^{*}\right]\{\mathrm{U}\}=\left[\mathrm{H}^{*}\right]^{\mathrm{T}}\left[\mathrm{G}^{*}\right]\{\mathrm{P}\}$

onde

$\left[\mathrm{H}^{*}\right]^{\mathrm{T}}$ tem ordem $((\mathrm{K} * \mathrm{nn}) \mathrm{X}(\mathrm{K} * \mathrm{nn}+2 * \mathrm{n} * \mathrm{ne}))$

$\left[\mathrm{H}^{*}\right]$ e $\left[\mathrm{G}^{*}\right]$ são de ordem $((\mathrm{K} * \mathrm{nn}+2 * \mathrm{n} * \mathrm{ne}) \mathrm{X}(\mathrm{K} * \mathrm{nn}))$

sendo $\{\mathrm{U}\}$ e $\{\mathrm{P}\}$ os do problema original de ordem $\left(\left(\mathrm{K}^{*} \mathrm{nn}\right) \mathrm{X}(1)\right)$

K para aproximação linear vale 2, pois o número de equações a ser gerado no caso linear é de duas vezes nn que é o número de nós, ne é o número de elementos do problema. $\mathrm{E}$ finalmente $\mathrm{n}$ é o número de pontos fontes adicionais por elemento a serem gerados. 
Para deixar mais clara a aplicação do método, será apresentado um exemplo bem simplificado, cujo contorno é discretizado com três elementos, uma única sub-região e o número de pontos fontes adicionais por elemento a ser empregado para aplicação da técnica neste caso é 1, mas poderia ser quantos se desejasse adicionar. A seguir é apresentada a discretização do problema:

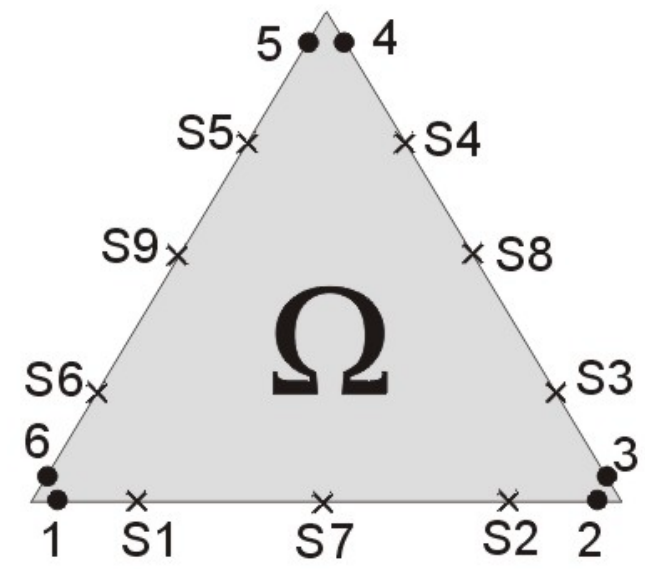

Figura 19 - Discretização do problema.

$\mathrm{Na}$ figura acima, se fossemos resolver este problema, necessitaríamos apenas dos pontos fontes S1, S2, S3, S4, S5 e S6 o que resultaria em uma matriz [H] e uma [G] de ordem $12 \times 12$ como é apresentado a seguir:
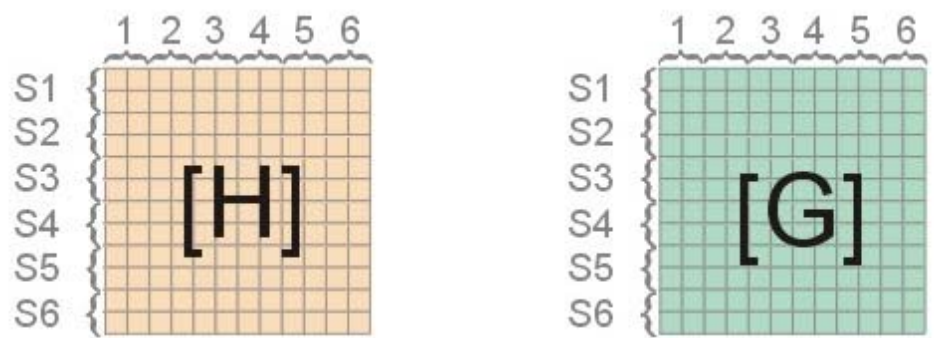

Figura 20 - Matrizes $[\mathrm{H}]$ e $[\mathrm{G}]$ originais que serão substituídas pelas novas matrizes $[\mathrm{H}]_{\text {Novo }}$ $\mathrm{e}[\mathrm{G}]_{\mathrm{Nov}}$.

Contudo para aplicar a suavização do contorno pela técnica dos mínimos quadrados por sub-região é necessário gerar mais equações do que incógnitas adicionando-se para isso os pontos fontes $\mathrm{S} 7, \mathrm{~S} 8$ e S9, criando-se assim as matrizes $\left[\mathrm{H}^{*}\right]$ e $\left[\mathrm{H}^{*}\right]^{\mathrm{T}}$. Logo a partir destas é gerado uma nova matriz $[\mathrm{H}]_{\text {Novo }}$ que substituirá no sistema original a matriz $[\mathrm{H}]$. 

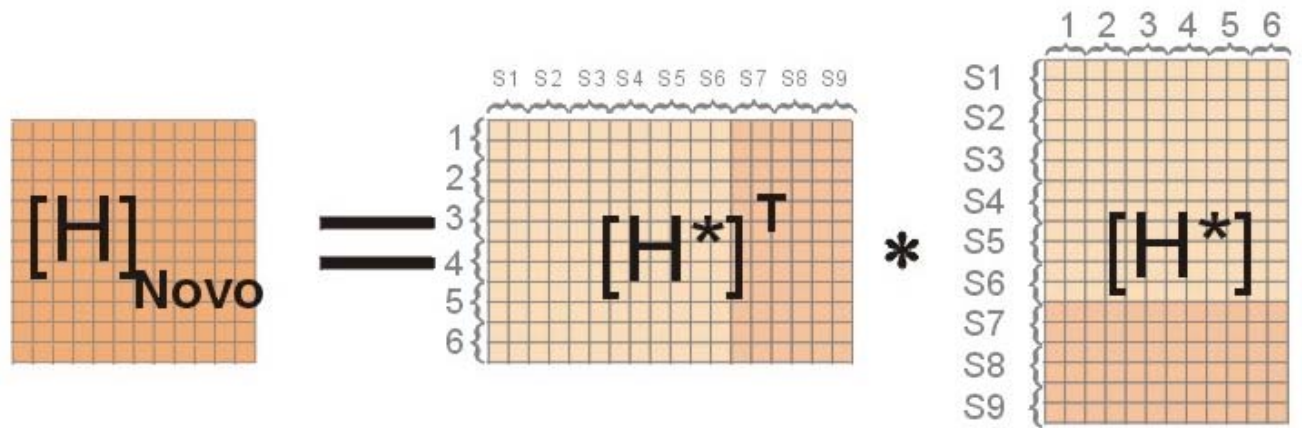

Figura 21 - Ilustração da montagem da nova matriz $[\mathrm{H}]_{\text {Novo }}$.

Para a criação da nova matriz $[\mathrm{G}]_{\text {Novo }}$ que substituirá a antiga $[\mathrm{G}]$ no sistema original, procedimento semelhante ao que aplicado na matriz $[\mathrm{H}]$ é empregado, contudo, a matriz transposta empregada é a matriz $\left[\mathrm{H}^{*}\right]^{\mathrm{T}}$, como é apresentado a seguir.
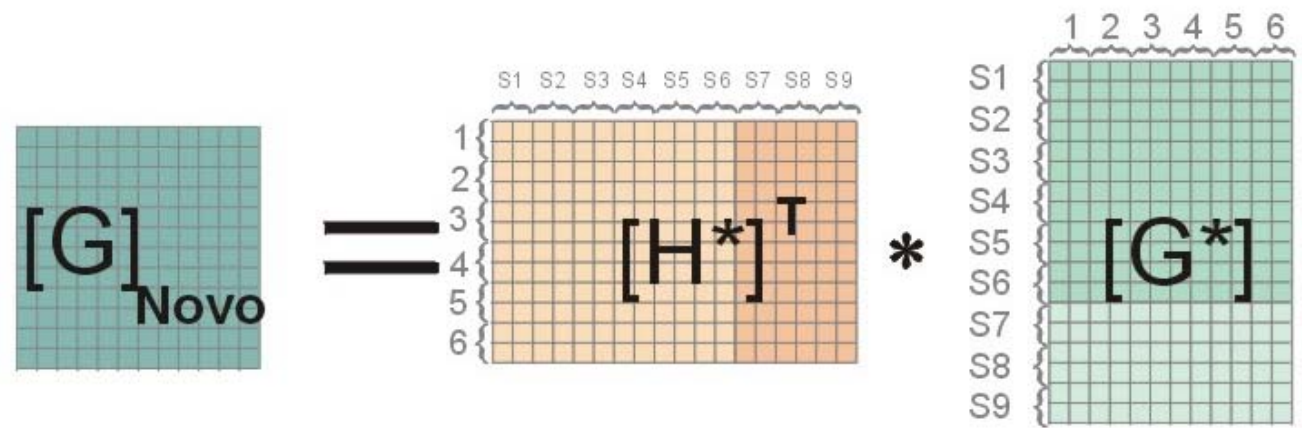

Figura 22 - Ilustração da montagem da nova matriz $[\mathrm{G}]_{\text {Novo }}$.

\subsection{MíNIMOS QUADRADOS POR ELEMENTO}

A técnica dos mínimos quadrados pode ser aplicada por elemento para suavização do contorno apresentando bons resultados e com um ganho considerável de tempo de processamento. Com no processo anterior, deve-se gerar mais equações inserindo-se novos pontos fontes. Só que agora a redução é feita somente entre as equações criadas para um único elemento como é descrito a seguir:

Partindo-se dos conjuntos de equações gerados pelos pontos fontes provenientes de um único elemento que podem ser representadas pelas parcelas das matrizes $[\mathrm{H}]$ e $[\mathrm{G}]$, aqui chamados de $[\mathrm{h}]$ e $[\mathrm{g}]$ tem-se:

$[\mathrm{h}]_{\text {novo }}=\left[\mathrm{h}^{* *}\right]^{\mathrm{T}}[\mathrm{h} *] \quad$ e $\quad[\mathrm{g}]_{\text {novo }}=\left[\mathrm{h}^{* *}\right]^{\mathrm{T}}[\mathrm{g} *]$ 
onde

$[\mathrm{h}]_{\text {novo }}$ e $[\mathrm{g}]_{\text {novo }}$ serão as novas parcelas que irão substituir as parcelas antigas $[\mathrm{h}] \mathrm{e}$ [g] em $[\mathrm{H}]$ e $[\mathrm{G}]$

$$
\begin{aligned}
& {\left[\mathrm{h}^{*}\right] \text { e }\left[\mathrm{g}^{*}\right] \text { de ordem }((\mathrm{K}+2 * \mathrm{n}) \mathrm{X}(\mathrm{K} * \mathrm{nn}))} \\
& {\left[\mathrm{h}^{* *}\right]^{\mathrm{T}} \text { é de ordem }((\mathrm{K} * \mathrm{nn}) \mathrm{X}(\mathrm{K}+2 * \mathrm{n}))}
\end{aligned}
$$

De forma semelhante à técnica de suavização por sub-região que foi apresentada anteriormente, será apresentada uma ilustração para deixar claro a aplicação da técnica. A discretização do problema é a mesma do exemplo anterior como pode ser observado na figura a baixo, o número de pontos fontes adicionais por elemento também é o mesmo.

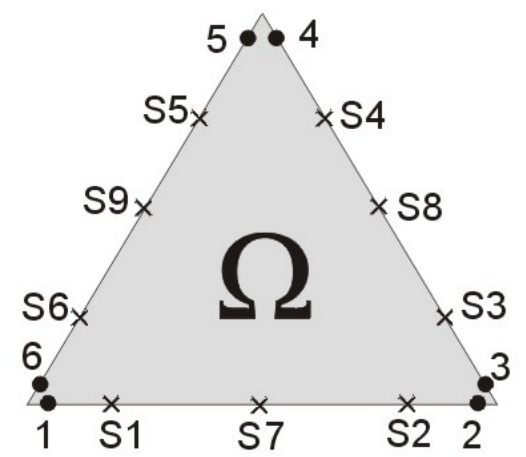

Figura 23 - Discretização do problema.

As matrizes $[\mathrm{H}]$ e $[\mathrm{G}]$ do sistema original seriam as mesmas do exemplo anterior como é ilustrado a seguir:
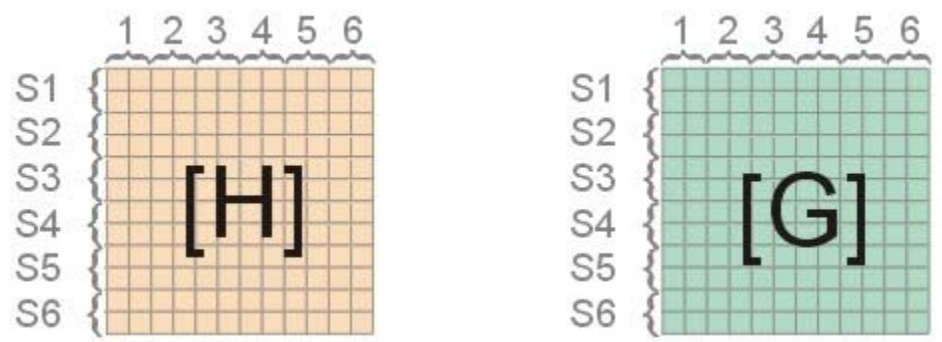

Figura 24 - Matrizes $[H]$ e $[\mathrm{G}]$ originais que serão substituídas pelas novas matrizes $[\mathrm{H}]_{\text {Novo }}$ $\mathrm{e}[\mathrm{G}]_{\text {Novo }}$.

Contudo, as novas matrizes $[\mathrm{H}]_{\text {Novo }}$ e $[\mathrm{G}]_{\text {Novo }}$ que irão substituir respectivamente as matrizes $[\mathrm{H}]$ e $[\mathrm{G}]$ serão criadas de outra forma como é ilustrado a seguir: 


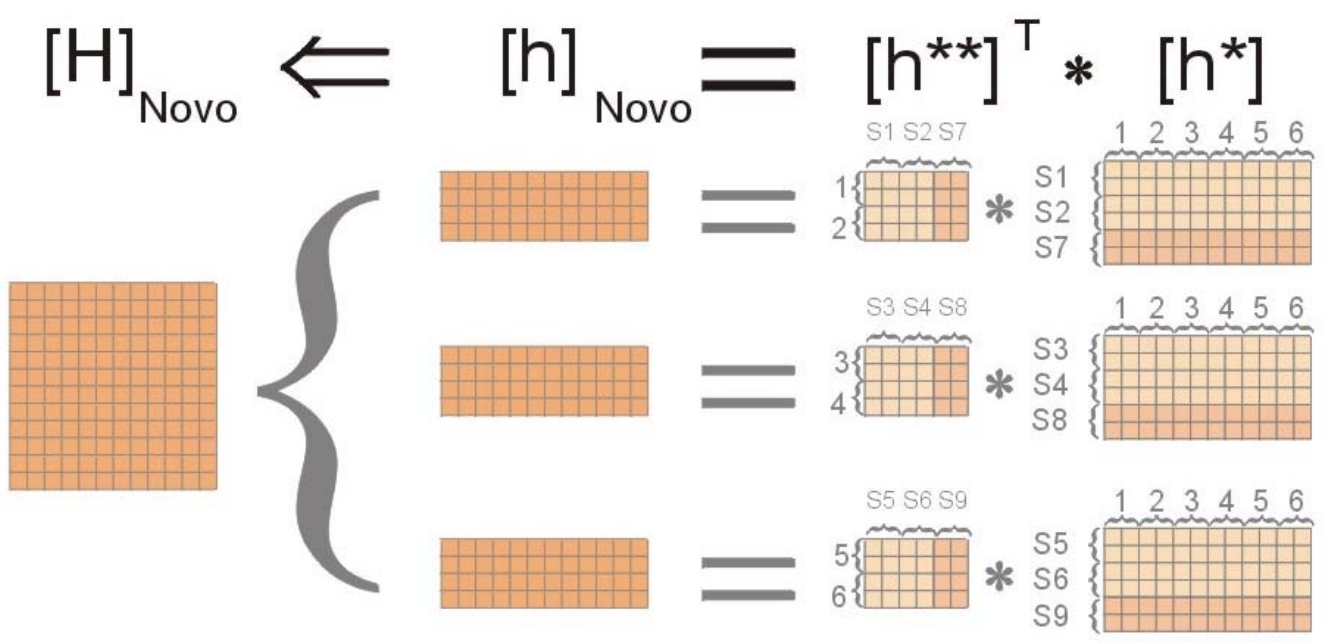

Figura 25 - Ilustração da montagem da nova matriz $[\mathrm{H}]_{\text {Novo }}$.

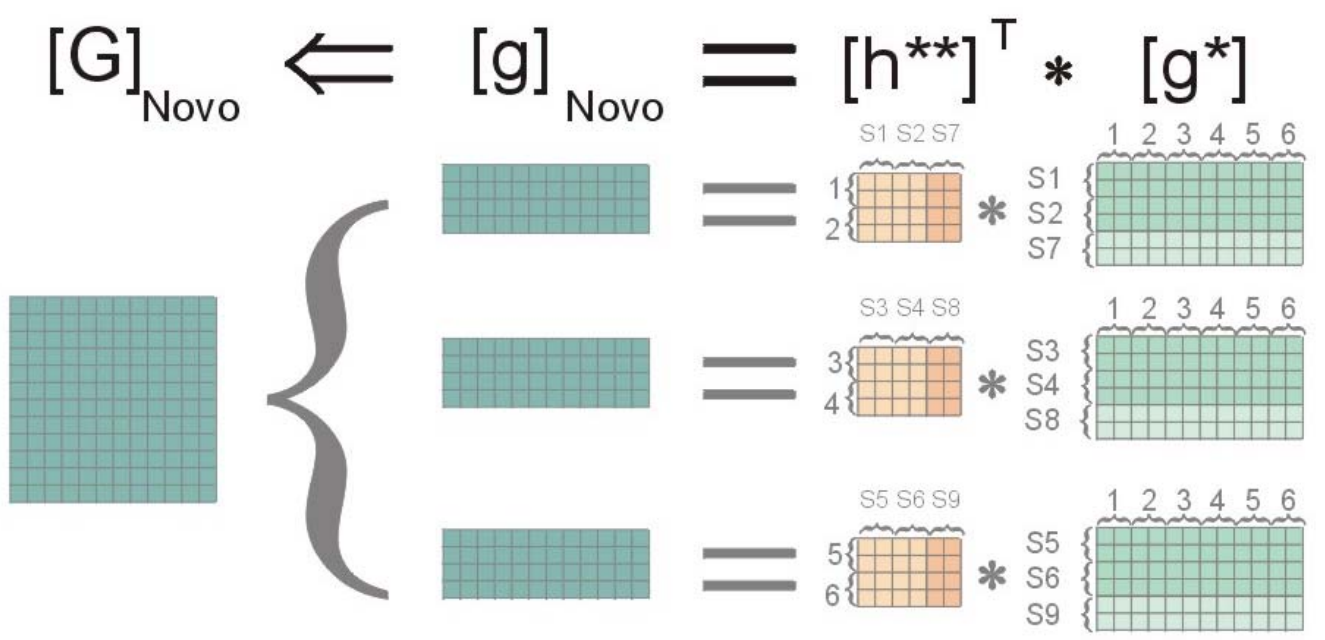

Figura 26 - Ilustração da montagem da nova matriz $[\mathrm{G}]_{\text {Novo }}$.

\subsection{EXEMPlOS DE APLICAÇÃo}

A seguir são descritos três exemplos para demonstrar as resultados da técnica. A primeiro exemplo trata-se de uma viga engastada com carga na ponta do balanço. Já no segundo exemplo estuda uma chapa retangular engastada em um dos lados e parcialmente engastada em outros dois e carregada na quarta face. E por último, os resultados de uma chapa composta por três domínios com módulos de elasticidade e coeficientes de Poisson diferentes, apresentando assim os resultados da técnica quando aplicada a mais de uma subregião. 


\section{Exemplo 1 Viga engastada}

Viga engastada com carga concentrada na ponta do balanço como é apresentado na figura 27 abaixo.

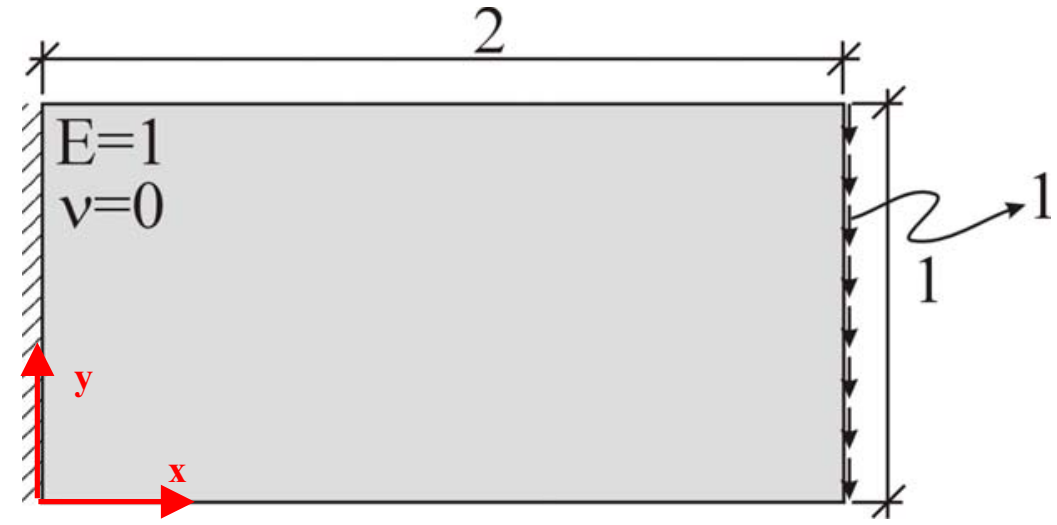

Figura 27 - Dados do exemplo.

As figuras 28 e 29 apresentam as discretizações adotadas neste exemplo, quando houver nó simples a simbologia adotada é apenas uma barra e no caso de nó duplo uma cruz. Os nós duplos serão empregados sempre que houver mudanças ou de vinculação ou de carregamentos.

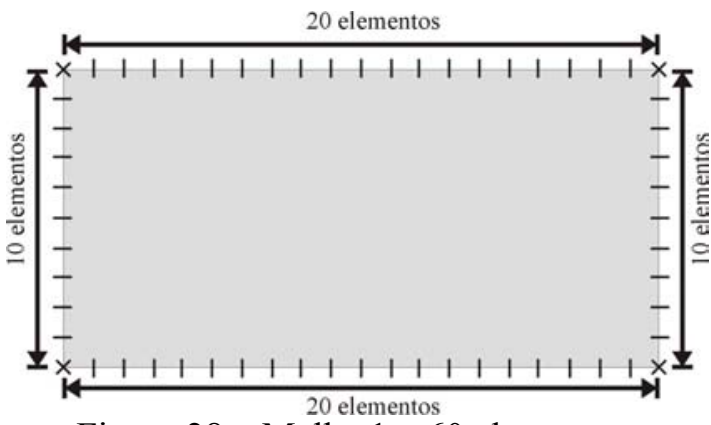

Figura 28 - Malha $1-60$ elementos.

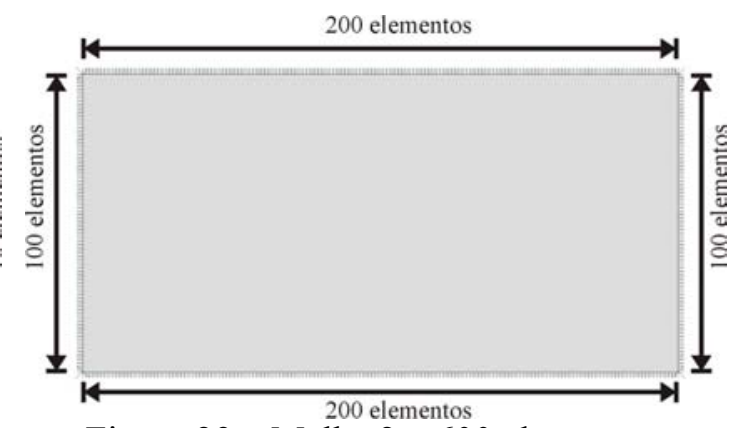

Figura 29 - Malha 2 - 600 elementos.

Outras discretizações foram simuladas, mas neste estudo apenas estes dois casos são estudados.

No gráfico 1 são apresentados os deslocamentos da face inferior da viga, para a malha com 60 elementos, sendo que as siglas SMQ 60 significa que esta linha representa os dados obtidos para simulação sem mínimos quadrados com 60 elementos, já MQE 60 é o caso onde foi empregada a técnica de mínimos quadrados por elemento para a malha de 60 
elementos, na MQS 60 a técnica é aplicada por sub-região novamente para a malha de 60 elementos de contorno.

Estes resultados são comparados com o caso SMQ 600 onde não é aplicada a técnica de mínimos quadrados, mas pela alta discretização obtém bons resultados. Ainda são apresentados os resultados teóricos muito conhecidos na literatura como viga de Bernoulli e viga de Timoshenko sendo que este último apresenta melhores resultados por levar em conta em sua formulação o efeito da tensão cisalhante.

Para cada elemento de contorno foram adicionados 4 pontos fontes a mais para a aplicação da técnica. Lembrando que este aumento do número de equações não influirá no tempo de resolução do sistema, pois o número de equações finais do sistema a ser resolvido não é alterado. Contudo o tempo de pré-processamento, ou seja, o tempo de integração e montagem das matrizes $\mathrm{H}$ e $\mathrm{G}$ dependendo da ordem do sistema é aumentado.

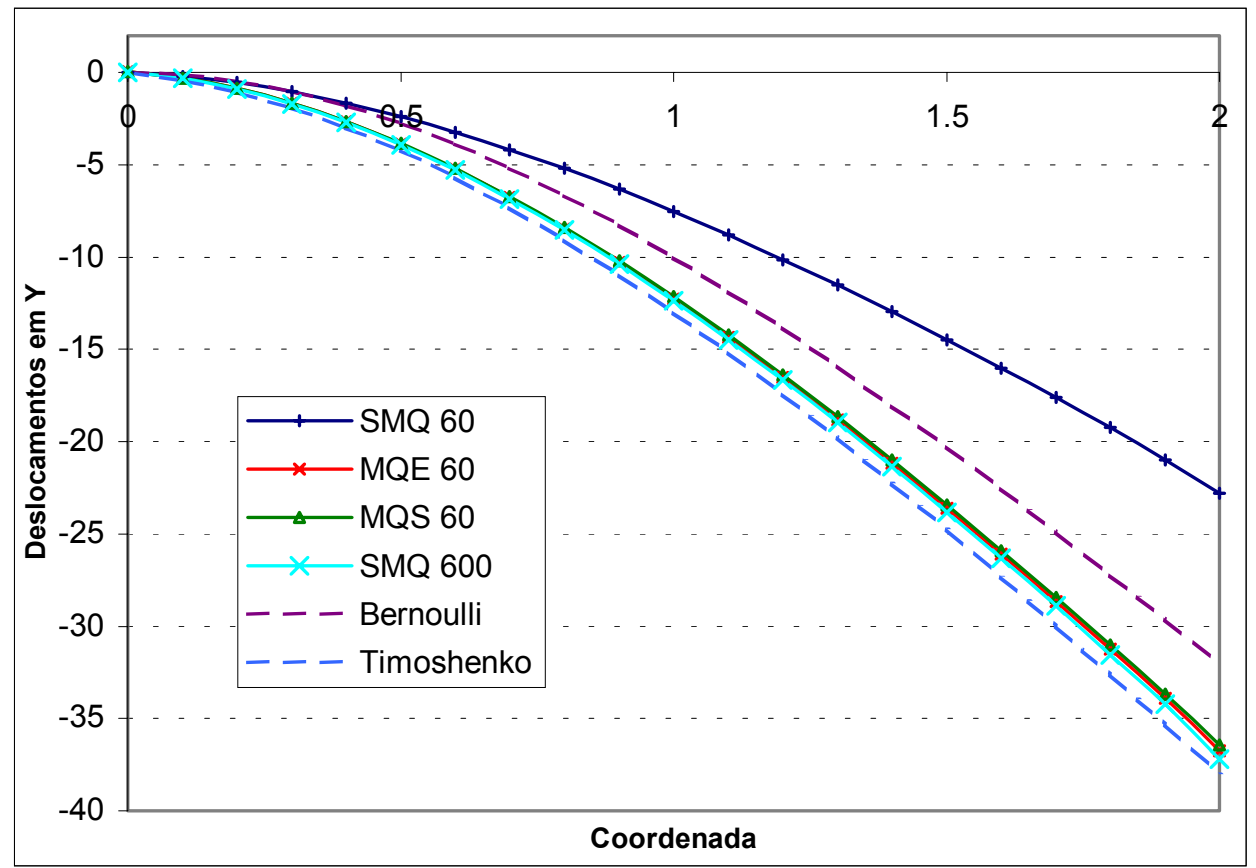

Gráfico 1 - Deslocamentos da face inferior da viga.

Como se pode observar, os deslocamentos dos casos MQE 60 e MQS 60 estão bem mais corretos dos que os do caso SMQ60. Esta mesma performance pode ser observada nos gráficos a seguir. No Gráfico 2 e 3 são apresentadas respectivamente as reações de apoio em $x$ e em $y$. 


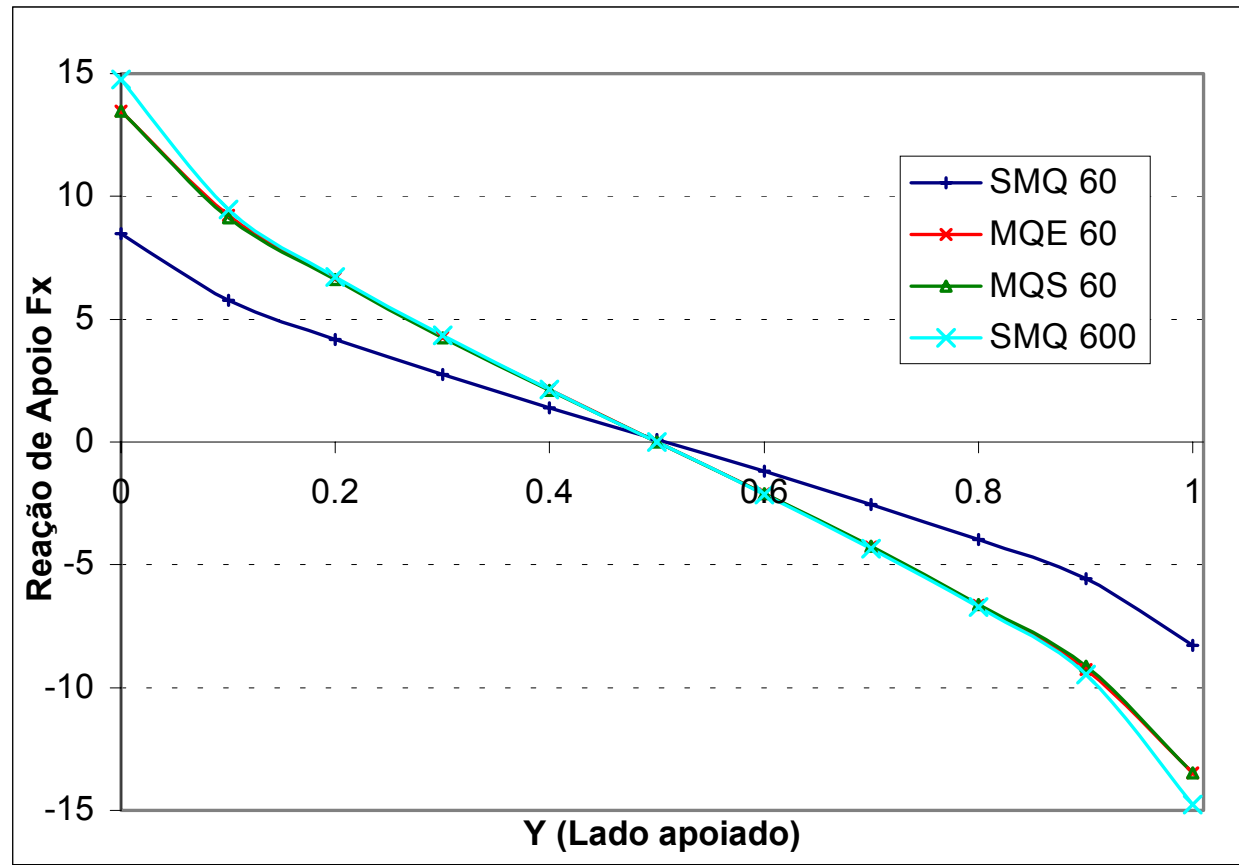

Gráfico 2 - Reação de apoio Fx do lado engastado.

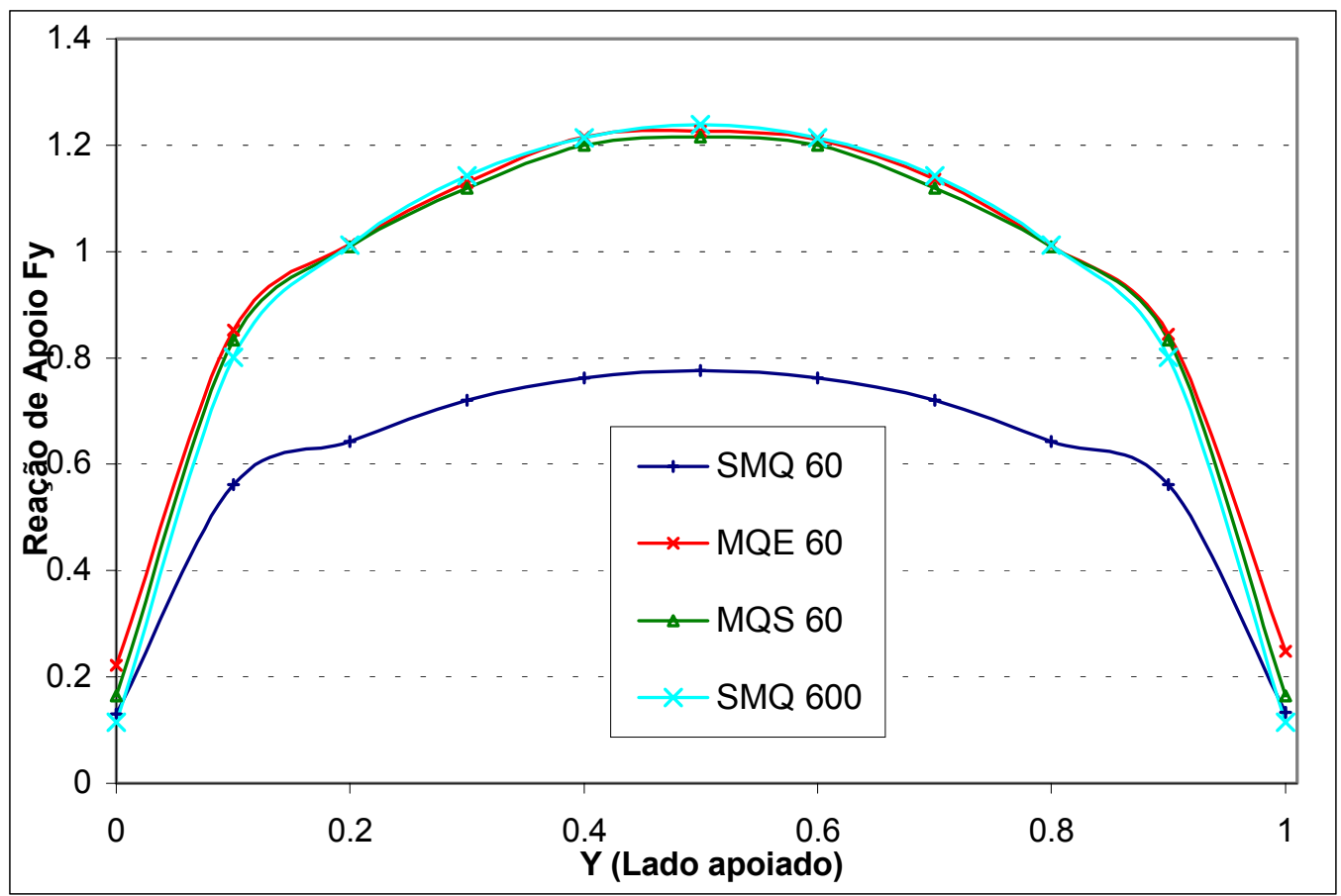

Gráfico 3 - Reação de apoio Fy do lado engastado.

Com o intuito de assegurar a qualidade dos resultados, é comparado o deslocamento da ponta do balanço (flecha) com os resultados obtidos por: cálculo teórico (tanto Bernoulli como Timoshenko), resultados obtidos através de simulação no programa Ansys 5.5 usando para isso 1296 elementos finitos quadrilaterais Shell 93, os resultados são ainda comparadas Formulação do Método dos Elementos de Contorno para Análise de Chapas com Enrijecedores. Wilson Wesley Wutzow 
com um programa que esta sendo desenvolvido por LOVÓN (2003) que possibilita análise auto-adaptativa através do método dos elementos de contorno com aproximação linear cujo parâmetro de iteração é a norma de energia total do sistema. Os resultados para a flecha na ponta do balanço podem ser observados na tabela 01 .

\begin{tabular}{lc}
\hline Método de cálculo & Deslocamento Y (cm) \\
\hline SMQ 60 & $-22,7958$ \\
MQS 60 & $-36,4392$ \\
MQE 60 & $-36,7334$ \\
SMQ 600 & $-37,1781$ \\
SHELL 93 (ANSYS) & $-36,7329$ \\
Adapt (E energ $<\mathbf{0 , 5} \%)_{\text {Bernoulli (teórico) }}$ & $-37,1623$ \\
Timoshenko (teórico) & $-32,0000$ \\
\hline
\end{tabular}

Tabela 01 - Deslocamentos em y da ponta do balanço.

Com esta comparação fica claro observar a qualidade dos resultados obtidos através da técnica de mínimos quadrados aplicado por elemento que supera em muito os resultados obtidos sem a técnica.

\section{Exemplo 2 Chapa engastada em um lado e parcialmente engastado em outros dois}

Neste exemplo são apresentados os resultados duma chapa retangular parcialmente engastada em duas faces e engastada numa outra, sendo a última carregada como é descrito na figura 30.

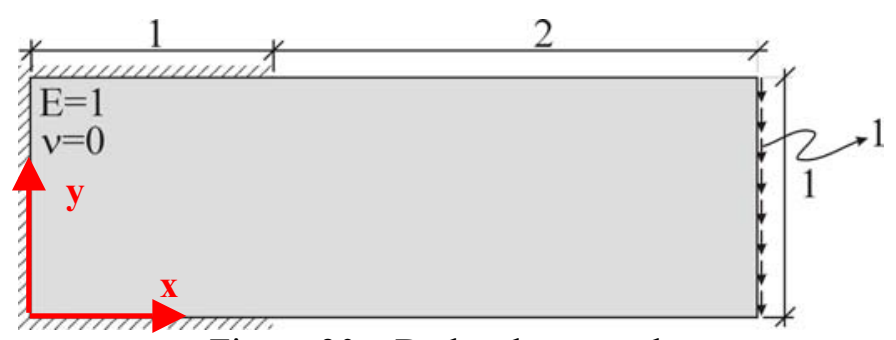

Figura 30 - Dados do exemplo. 
Por estarmos estudando apenas a eficiência da formulação, não preocupou-se em estudar casos reais, adotando-se então valores fictícios de módulo de elasticidade, coeficiente de Poisson, cargas e dimensões da peça.

As figuras 31,32, apresentam respectivamente as duas discretizações adotadas neste exemplo.

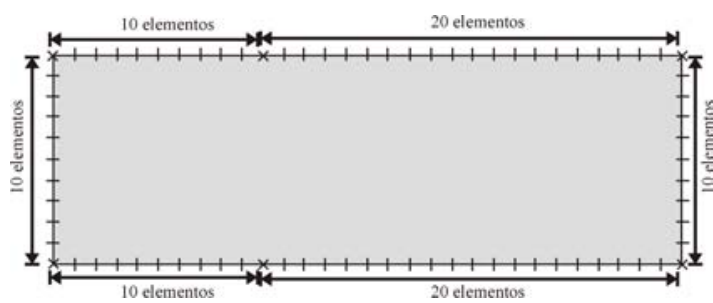

Figura 31 - Malha $1-80$ elementos.

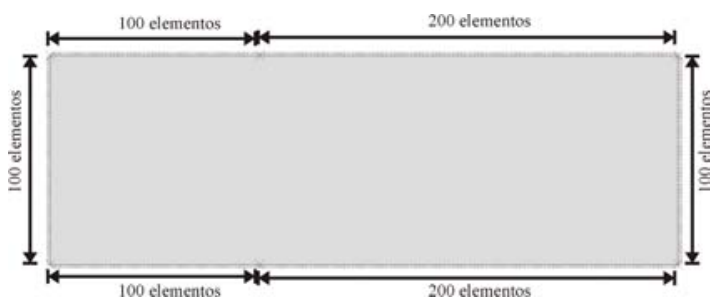

Figura 32 - Malha $1-800$ elementos.

Assim como no exemplo anterior, o gráfico da flecha da face inferior da viga, e os gráficos de reação de apoio do lado totalmente engastado são apresentados respectivamente nos gráficos 4,5 e 6 .

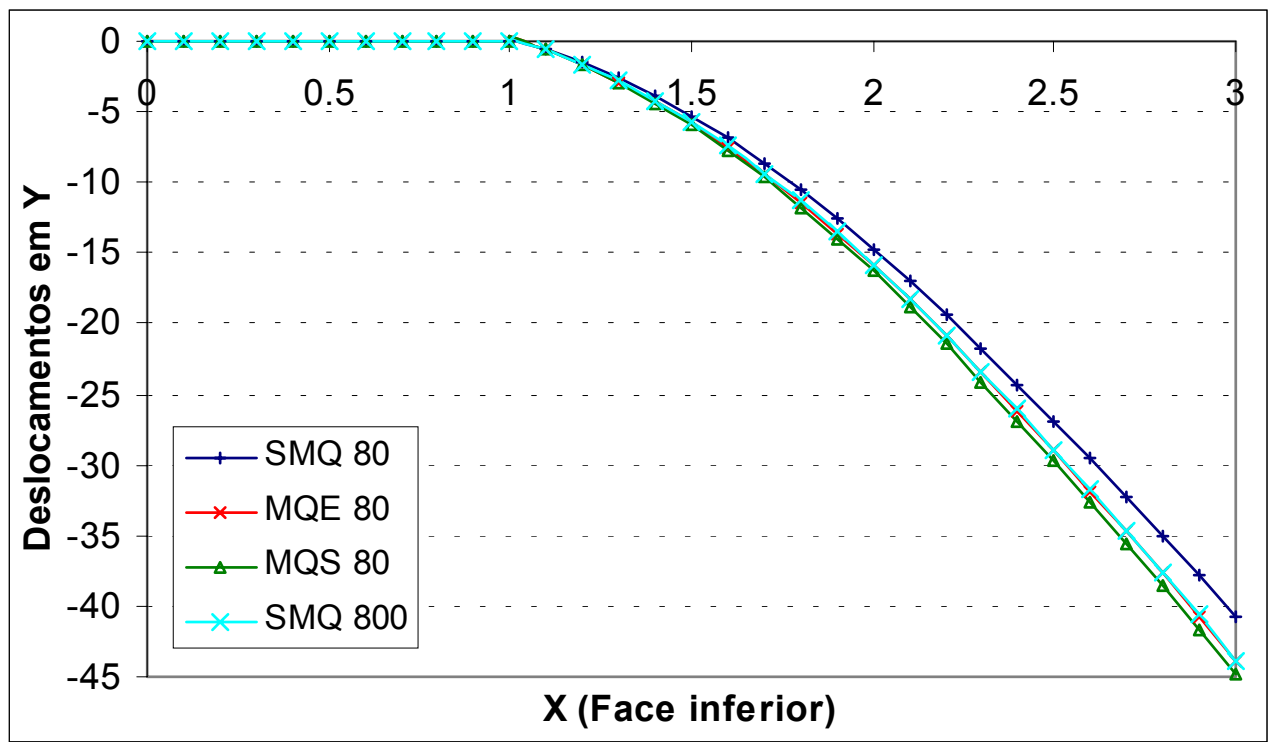

Gráfico 4 - Deslocamentos da face inferior.

Neste exemplo apesar dos resultados não serem tão expressivos para os casos em que é aplicada a técnica de mínimos quadrados, ainda assim pode-se observar um ganho de performance. 


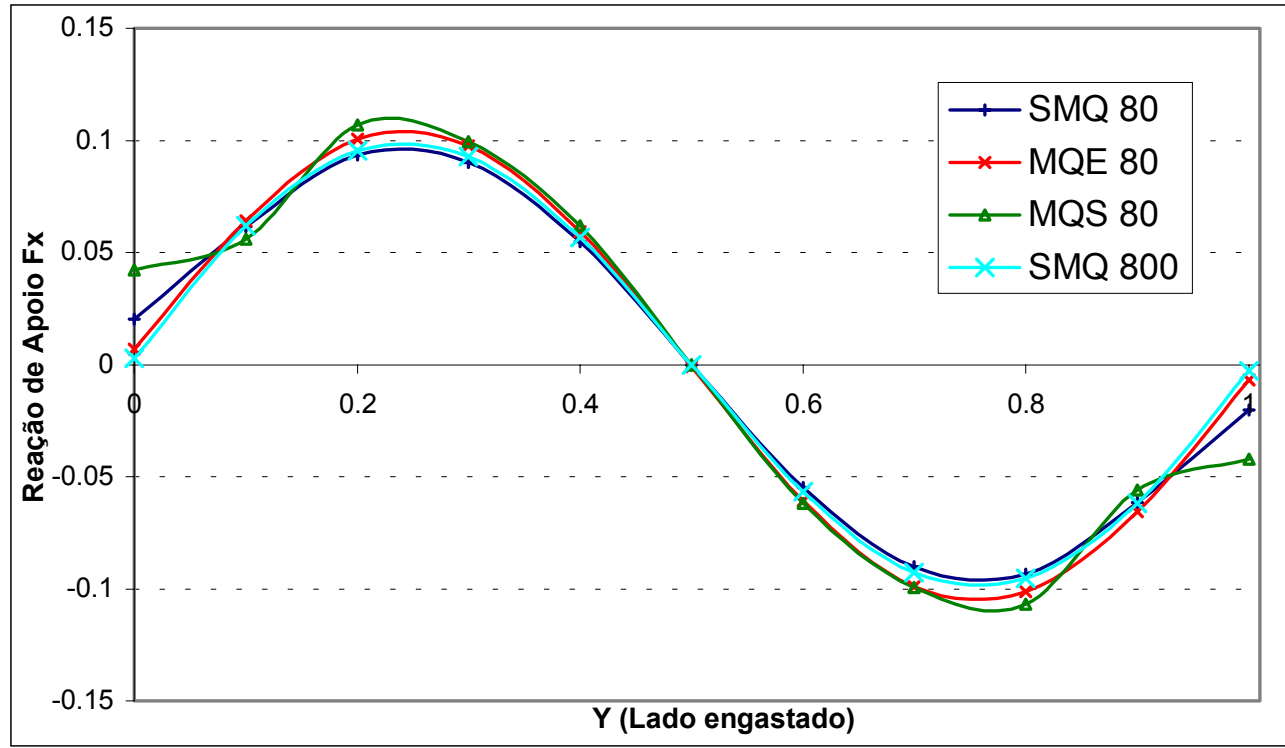

Gráfico 5 -Reação de apoio Fx do lado totalmente engastado.

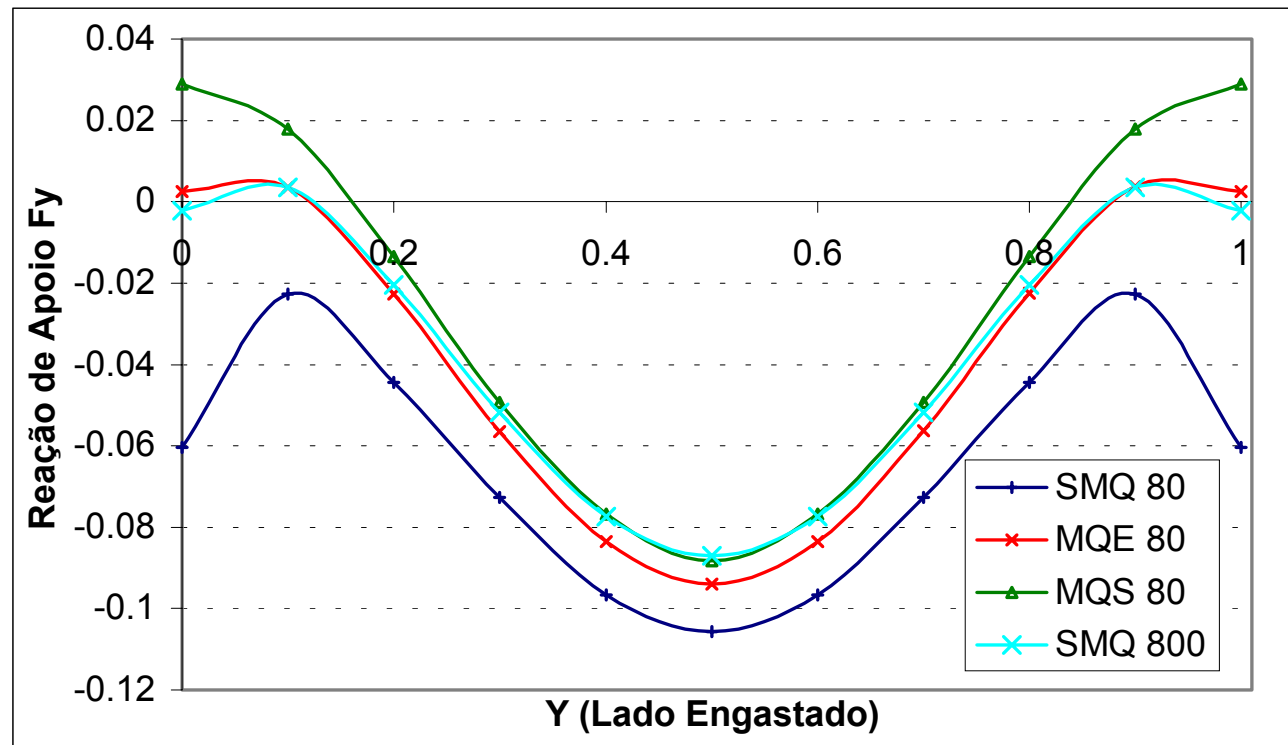

Gráfico 6 -Reação de apoio Fy do lado totalmente engastado.

Neste exemplo, assim como no anterior, os resultados obtidos são comparados com resultados encontrados por outras formas de cálculo, pode-se observar na tabela 02 uma comparação do os valores da flecha na extremidade calculados tanto pelos modelos estudados nesta dissertação como os resultados obtidos por LOVÓN(2003) e através do programa ANSYS 5.5 com o elemento quadrilateral Shell 93 (3600 elementos). 


\begin{tabular}{lc}
\hline Método de cálculo & Deslocamento Y (cm) \\
\hline SMQ 80 & $-40,7504$ \\
MQS 80 & $-44,8369$ \\
MQE 80 & $-43,8486$ \\
SMQ 800 & $-43,8330$ \\
SHELL 93 (ANSYS) & $-42,2380$ \\
Adapt ( energ $_{\text {(0,5\%) }}$ & $-43,8532$ \\
\hline
\end{tabular}

Assim como no exemplo anterior, bons resultados são encontrados com a aplicação da técnica de suavização, podendo-se observar que com sua aplicação, não há necessidade de um refinamento excessivo da malha de contorno.

\section{Exemplo 3 Problemas com multi-regiões}

Neste exemplo é apresentado o resultado da aplicação da técnica de mínimos quadrados em um problema composto por mais de um domínio, tendo estes domínio características físicas diferentes. O problema trata-se de uma viga engastada subdividida em três domínios diferentes onde os domínios da extremidade do balanço e o domínio da outra extremidade engastada são compostos por materiais mais rígidos sendo então o domínio central mais flexível, como é apresentado na figura 33.

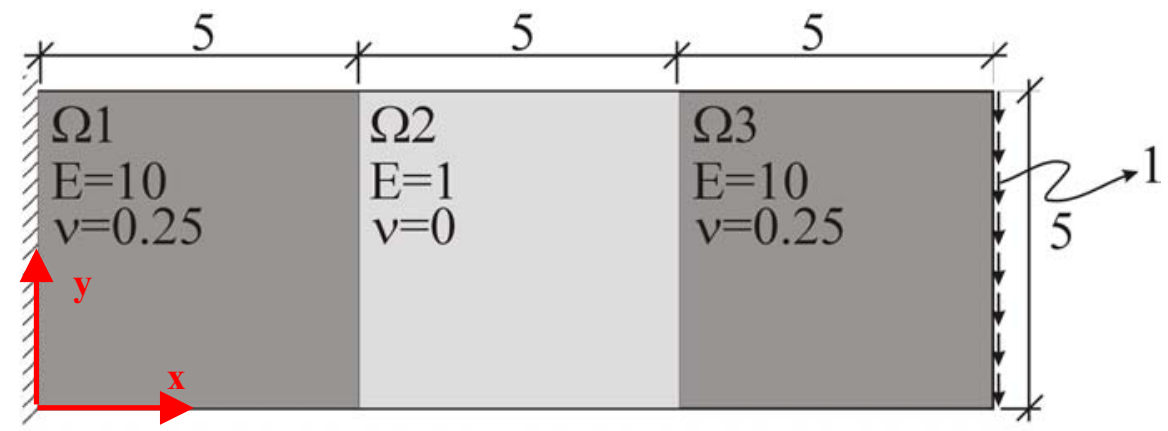

Figura 33 - Dados do exemplo.

Várias discretizações foram estudadas, mas serão apresentados os resultados de apenas duas, a menos discretizada era composta por 50 elementos de contorno, onde foram calculados os três casos: SMQ 50, MQE 50 e MQS 50, estes resultados são comparados com os apresentados pela segunda malha mais discretizada sendo composta por 500 elementos Formulação do Método dos Elementos de Contorno para Análise de Chapas com Enrijecedores. ～Wilson Wesley Wutzow 
sem a aplicação da técnica (SMQ 50). Estas malhas descritas podem ser observadas nas figuras 34 e 35 .

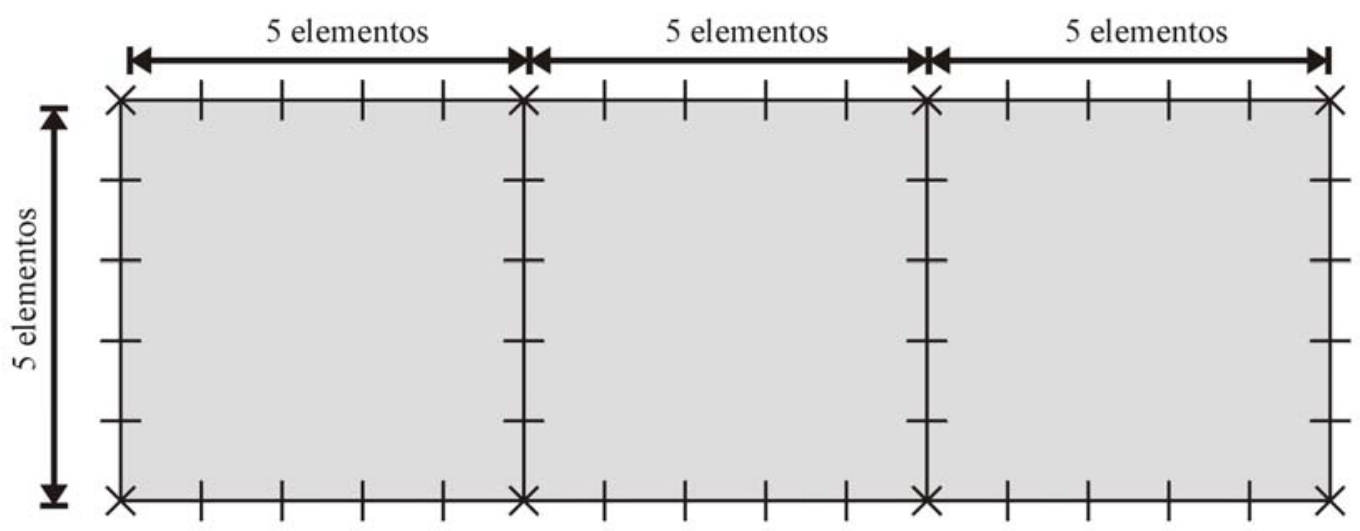

Figura $34-1^{\text {a }}$. Malha, composta por 50 elementos.

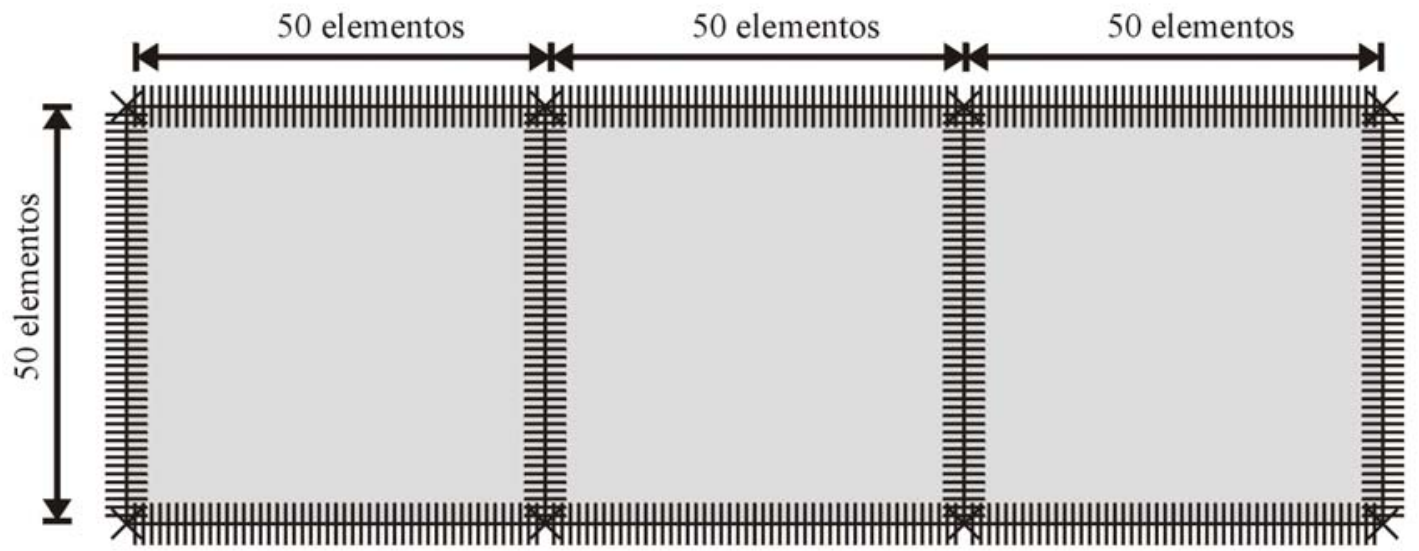

Figura $35-2^{\text {a }}$. Malha, composta por 500 elementos.

Para este exemplo, apenas alguns diagramas são apresentados, no gráfico 7 são apresentados os deslocamentos em y da face inferior da viga, já nos diagramas 8 e 9 são apresentados respectivamente os gráficos de reação em X e em Y na interface entre os domínio 1 e 2 . E por último nos diagramas 10 e 11 os deslocamentos desta mesma interface, primeiramente em X e depois em Y. 


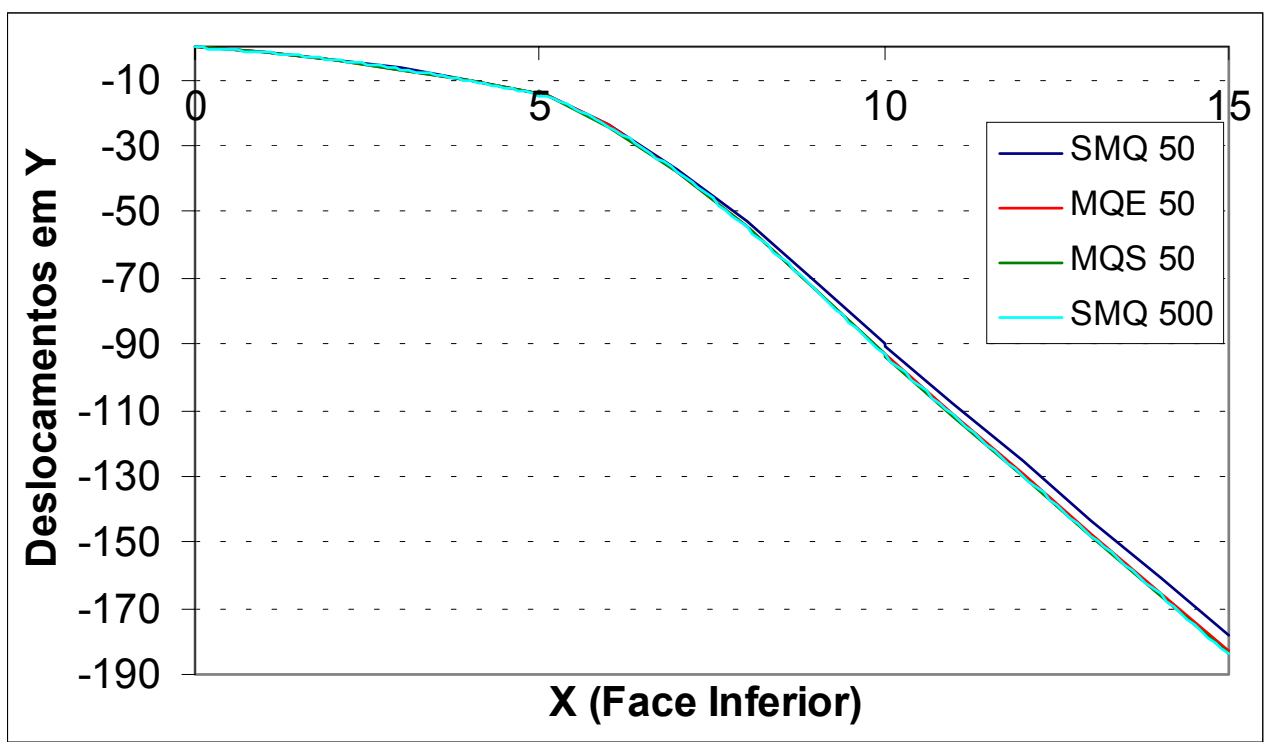

Gráfico 7 - Deslocamentos em Y da face inferior da viga.

No diagrama da flecha apresentada pela viga ainda é possível observar uma melhora nos resultados quando se aplica a técnica tanto por elemento como por sub-região.

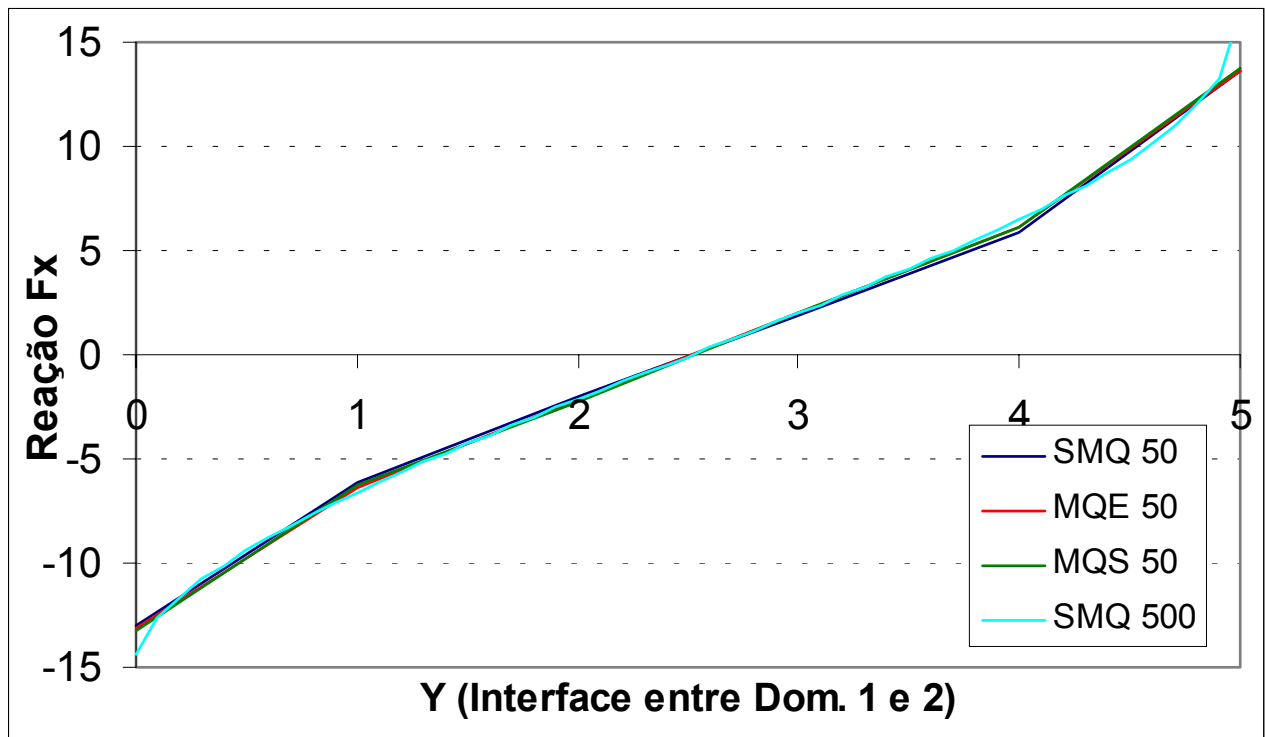

Gráfico 8 - Reações de apoio em X na interface entre os domínio 1 e 2 da viga. 


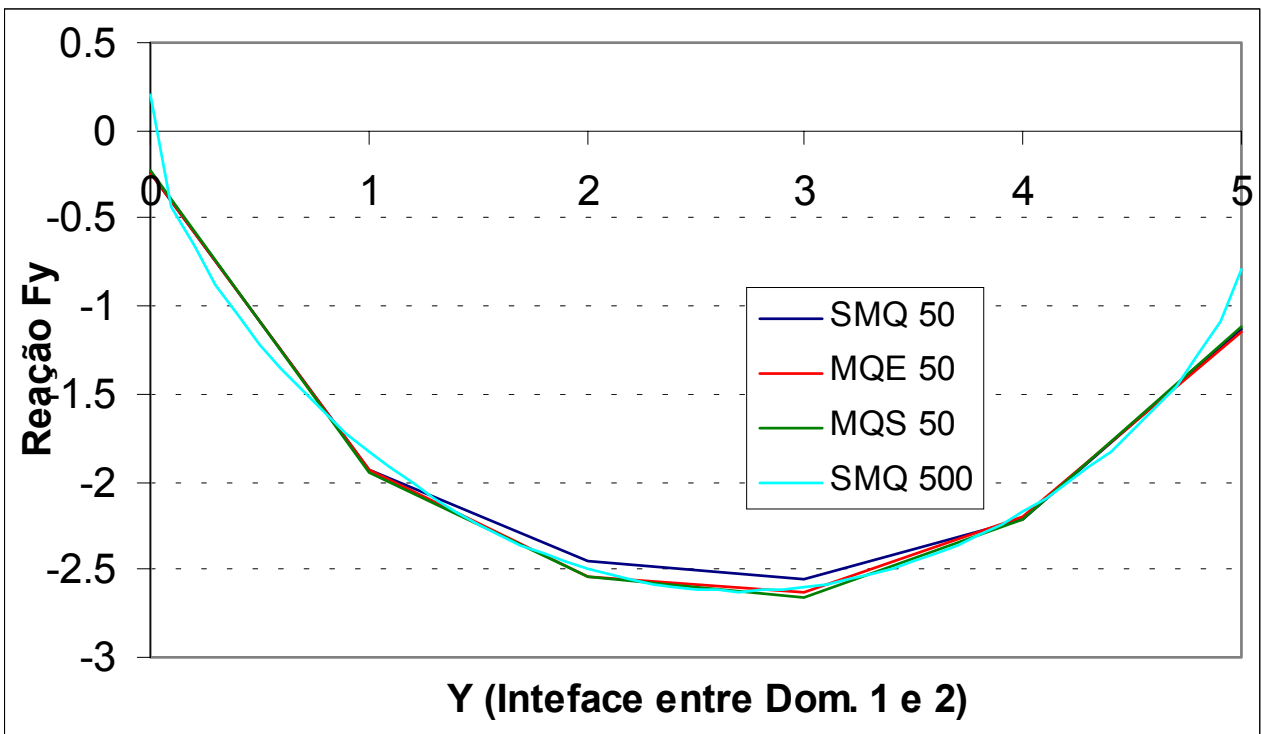

Gráfico 9 - Reações de apoio em Y na interface entre os domínio 1 e 2 da viga.

Apesar de se observar uma melhora nos resultados para os casos em que se aplica a técnica, nem sempre o ganho de performance é significativa, tanto nos diagrama de deslocamentos da interface como nos diagramas de forças na interface entre os domínio 1 e 2 o resultado melhora pouco ou quase nada se comparado com os casos em que a técnica é aplicada.

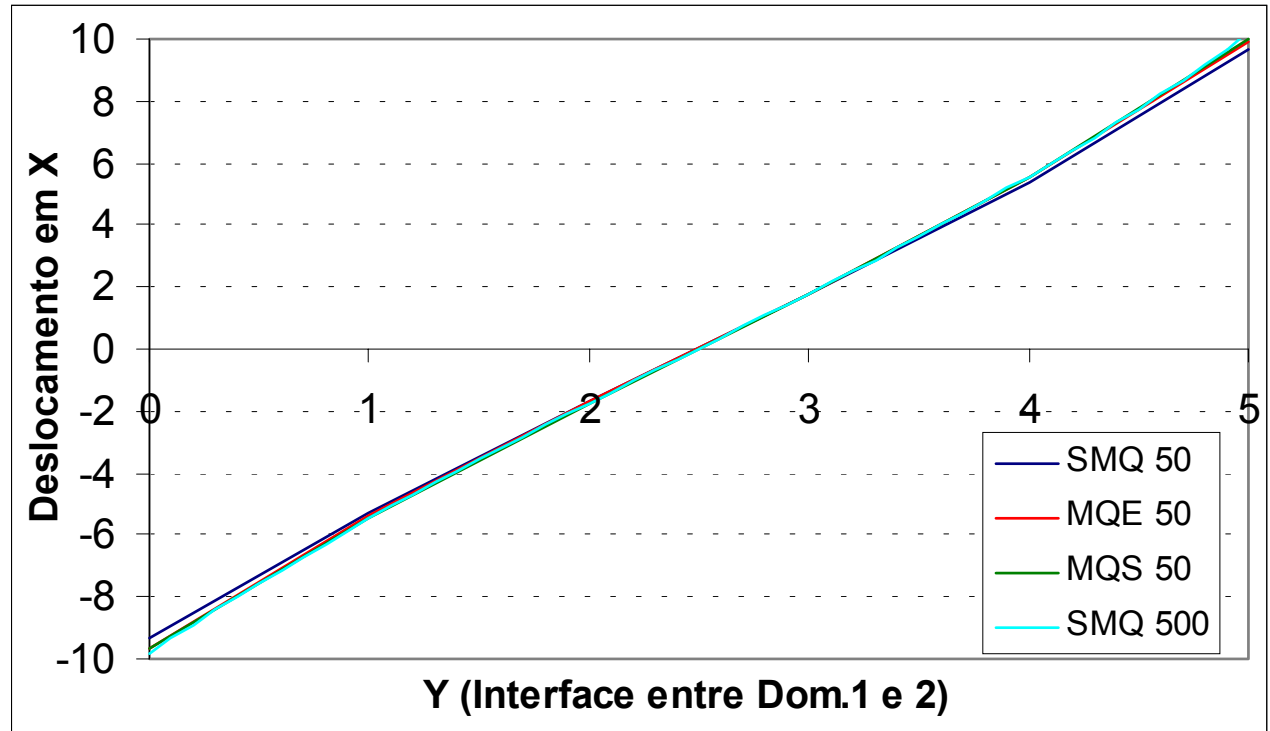

Gráfico 10 - Deslocamentos na direção X da interface entre os domínio 1 e 2 da viga. 


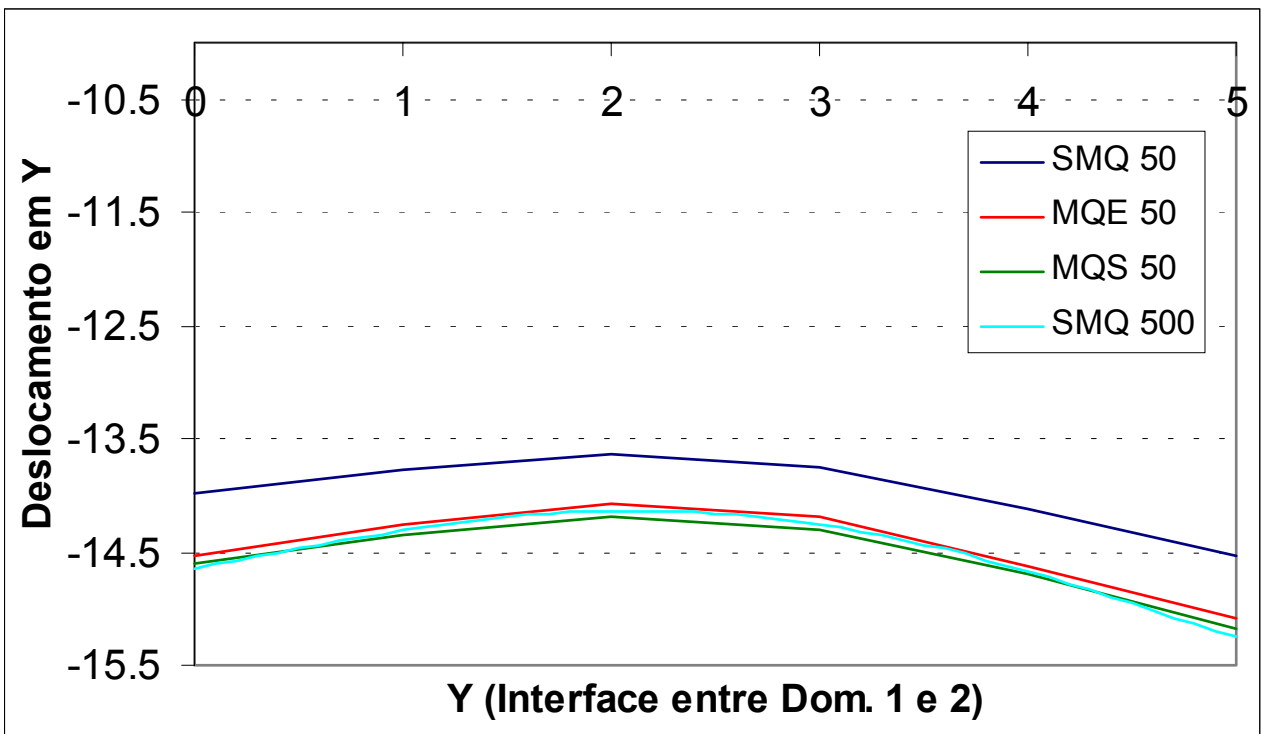

Gráfico 11 - Deslocamentos na direção Y da interface entre os domínio 1 e 2 da viga.

Os resultados são comparados com os obtidos pela técnica de sub-região simples (para uma discretização elevada) e com os resultados obtidos através da simulação com o programa ANSYS 5.5 utilizando-se 1875 elementos finitos Shell 93. Os resultados podem ser observados na tabela 03 .

\begin{tabular}{lc}
\hline Método de cálculo & Deslocamento Y (cm) \\
\hline SMQ 50 & $-178,3855$ \\
MQS 50 & $-184,0139$ \\
MQE 50 & $-183,2659$ \\
SMQ 500 & $-183,9921$ \\
SHELL 93 (ANSYS) & $-193,1930$
\end{tabular}

Tabela 03 - Deslocamentos em y da ponta do balanço.

\subsection{Conclusões parciais}

Aos estudarmos em implementarmos a técnicas podemos ponderar algumas conclusões. Não se pode negar que a técnica é eficiente, pois para uma grande quantidade de casos simulados (além dos casos em que foram apresentados os resultados, muitos outros Formulação do Método dos Elementos de Contorno para Análise de Chapas com Enrijecedores. $\quad$ Wilson Wesley Wutzow 
casos foram simulados) os resultados sempre, ou quase sempre os resultados convergiram para uma melhor resultado superior a aqueles apresentados quando a técnica não é aplicada. É bem verdade que nem sempre o ganho de performance seja significativo a ponto de justificar a aplicação da técnica.

Se comparados os resultados das duas formas de aplicação da técnica: por sub-região ou por elemento, na grande maioria dos casos quando se aplicava a técnica por elemento os resultados encontrados eram mais satisfatórios.

Resultados obtidos por outros pesquisadores BOTTA \& VENTURINI (2003) demonstram que esta técnica é muito útil no caso de suavização de resultados oscilantes, como por exemplo, aqueles que ocorrem no acoplamento MEC/MEF para discretizações pobres. 
Neste capítulo é apresentado o equacionamento empregado na representação dos enrijecedores através da técnica de sub-região.

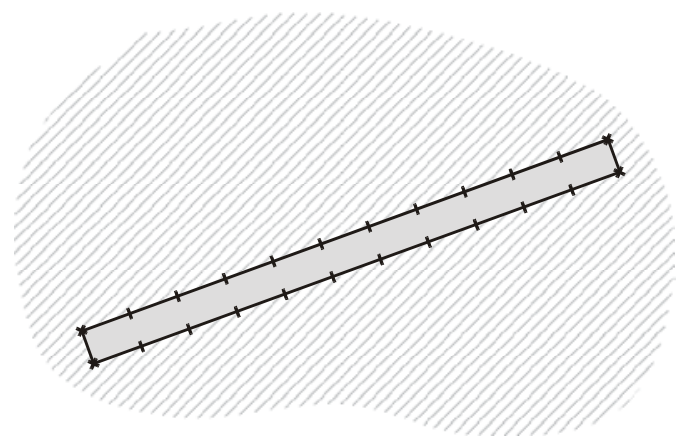

Figura 36 - Enrijecedor.

Os enrijecedores, elementos via de regra lineares de espessura quase sempre desprezível, são comumente representados no método dos elementos de contorno através do acoplamento MEC/MEF, onde o MEC é empregado para modelar o meio elástico contínuo e o MEF para representar elementos lineares rígidos (esta é uma entre várias aplicações deste tipo de acoplamento).

Com a melhoria da integração da solução fundamental de Kelvin através da integração analítica singular e não singular, e com a implementação da técnica de subregiões, uma alternativa viável para os enrijecedores é a sua representação através da utilização da técnica de sub-regiões, já que a qualidade das equações é garantida através da aplicação da integração analítica, juntamente com a suavização do contorno com o emprego 
da técnica de mínimos quadrados. Sendo ainda as incógnitas que seriam escritas no contorno podendo ser transformadas em incógnitas centrais, com redução ou não de número de incógnitas. Nesta dissertação é aplicada a técnica de sub-regiões com integração analítica e as incógnitas são apenas transformadas em incógnitas da linha central, sem haver redução do número de variáveis.

\subsection{SUb-REGIÕES}

Utilizando-se a técnica das sub-regiões, é possível resolver problemas cujos domínios sejam compostos por vários sub-domínios de materiais que representam características diferentes. Para equacionar este problema, discretiza-se isoladamente cada sub-domínio $\Omega_{\mathrm{i}}$ homogêneo. Com a imposição do equilíbrio das forças de superfície e da compatibilidade dos deslocamentos em todos os pontos de interface das sub-regiões, o equacionamento fica completo.

Considerando-se as forças de superfície e os deslocamentos dos pontos de interface como valores incógnitos, as equações são reunidas em um único sistema, que representa a característica de ser constituído por blocos nulos e não-nulos, formando uma matriz esparsa, sendo possível sua resolução, desde que a rotina utilizada para esta tarefa resolva sistemas esparsos. No programa desenvolvido, foi utilizada uma rotina do Fortran que emprega Gauss-Jordan com pivoteamento completo.

Para ilustrar esta técnica será apresentado um exemplo composto de duas subregiões, que está ilustrado na figura 37.

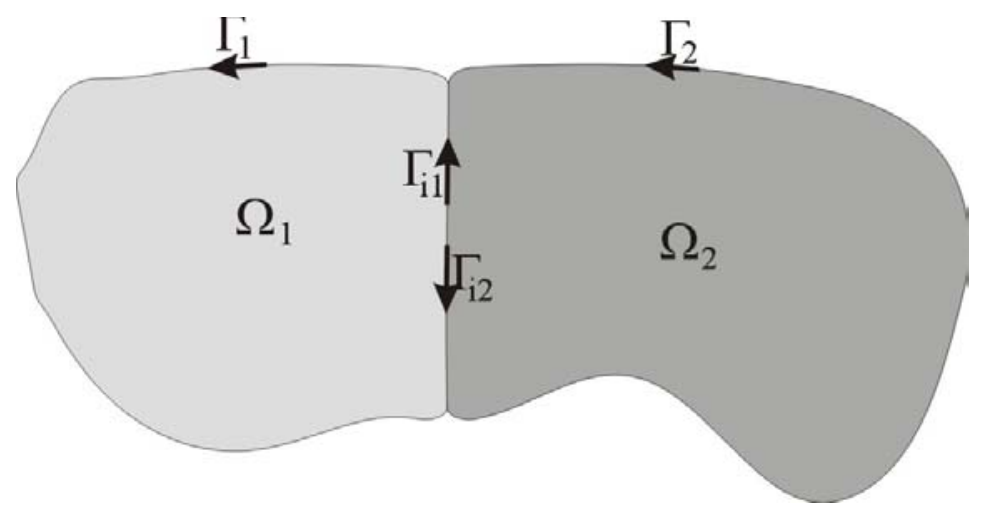

Figura 37 - Duas Sub-regiões. 
Sem considerar o equilíbrio das forças de superfície e compatibilidade de deslocamentos, pode-se escrever a seguinte representação matricial para os dois domínios de forma independente.

Para $\Omega_{1}$ tem-se:

$\left[\begin{array}{ll}{[H]_{11}^{1}} & {[H]_{1 i}^{1}} \\ {[H]_{i 1}^{1}} & {[H]_{i i}^{1}}\end{array}\right]\left\{\begin{array}{l}\{U\}^{1} \\ \{U\}^{1 i i}\end{array}\right\}=\left[\begin{array}{ll}{[G]_{11}^{1}} & {[G]_{1 i}^{1}} \\ {[G]_{i 1}^{1}} & {[G]^{1 i}}\end{array}\right]\left\{\begin{array}{l}\{P\}^{1} \\ \{P\}^{1 i i}\end{array}\right\}$

E em $\Omega_{2}$ tem-se:

$\left[\begin{array}{ll}{[\mathrm{H}]_{22}^{2}} & {[\mathrm{H}]_{2 \mathrm{i}}^{2}} \\ {[\mathrm{H}]_{\mathrm{i} 2}^{2}} & {[\mathrm{H}]_{\mathrm{ii}}^{2}}\end{array}\right]\left\{\begin{array}{l}\{\mathrm{U}\}^{2} \\ \{\mathrm{U}\}^{2 \mathrm{i}}\end{array}\right\}=\left[\begin{array}{ll}{[\mathrm{G}]_{22}^{2}} & {[\mathrm{G}]_{2 \mathrm{i}}^{2}} \\ {[\mathrm{G}]_{\mathrm{i} 2}^{2}} & {[\mathrm{G}]_{\mathrm{ii}}^{2}}\end{array}\right]\left\{\begin{array}{l}\{\mathrm{P}\}^{2} \\ \{\mathrm{P}\}^{2 \mathrm{i}}\end{array}\right\}$

Juntando os dois sistemas num só, sem considerar o equilíbrio das forças de superfície e compatibilidade de deslocamentos tem-se:

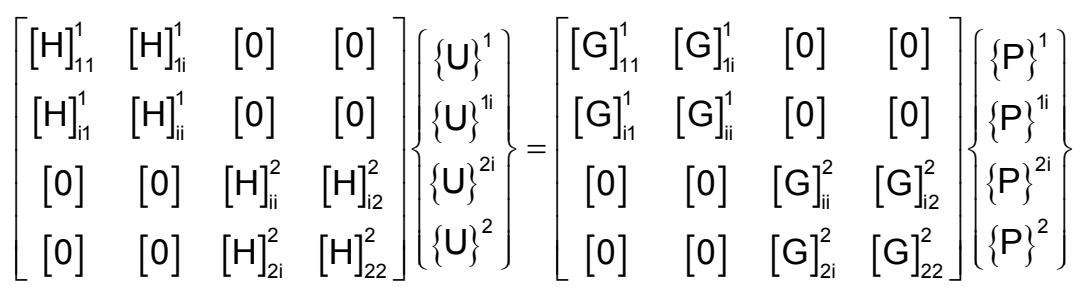

Para que o problema fique completamente determinado é necessário aplicar as condições de equilíbrio e de compatibilidade que podem ser facilmente descritas pelas seguintes equações:

Equação de equilíbrio das forças:

$\{P\}^{1 i}+\{P\}^{2 i}=0$

Equação de compatibilidade de deslocamentos:

$\{U\}^{1 i}=\{U\}^{2 i}$

Aplicada às condições de equilíbrio de forças e as de compatibilidade de deslocamentos chega-se ao seguinte sistema: 


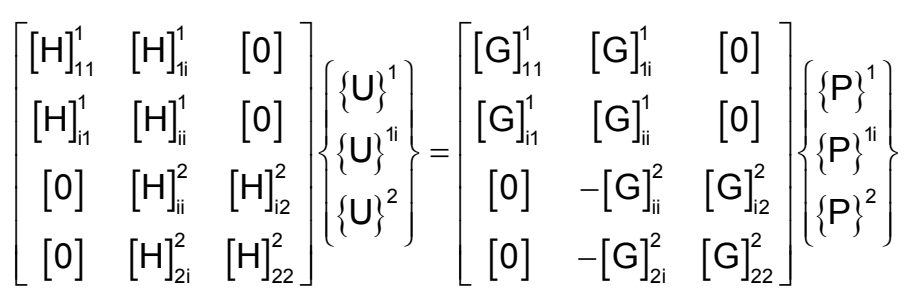

Após aplicar as condições de equilíbrio e compatibilidade, aplica-se as condições de contorno, que para sub-regiões este procedimento é feito de forma análoga ao procedimento feito em uma única sub-região, se for prescritos deslocamento, deve-se trocar a coluna equivalente a coluna do nó prescrito entre a matriz $[\mathrm{H}]$ e a matriz $[\mathrm{G}]$ e ainda deve-se trocar a linha equivalente ao nó prescrito entre os vetores $\{\mathrm{U}\}$ e $\{\mathrm{P}\}$.

Contudo deve-se ter em mente que da forma como o sistema foi escrito, só se pode prescrever condições de contorno para os nós que não pertencem às interfaces que conectam um domínio com outro. Pois para as interfaces entre dois domínios foi pressuposto que as duas variáveis (força de deslocamento) são incógnitas.

Para resolver o sistema deve-se ainda passar para o lado esquerdo todas as incógnitas de interface também. Deixando o sistema da seguinte maneira:

$$
\left[\begin{array}{cccc}
{[H]_{11}^{1}} & {[H]_{1 i}^{1}} & -[G]_{1 i}^{1} & {[0]} \\
{[H]_{i 1}^{1}} & {[H]_{i i}^{1}} & -[G]_{i i}^{1} & {[0]} \\
{[0]} & {[H]_{i i}^{2}} & {[G]_{i i i}^{2}} & {[H]_{i 2}^{2}} \\
{[0]} & {[H]_{2 i}^{2}} & {[G]_{2 i}^{2}} & {[H]_{22}^{2}}
\end{array}\right]\left\{\begin{array}{l}
\{U\}^{1} \\
\{U\}^{1 i} \\
\{P\}^{1 i} \\
\{U\}^{2}
\end{array}\right\}=\left[\begin{array}{cc}
{[G]_{11}^{1}} & {[0]} \\
{[G]_{i 1}^{1}} & {[0]} \\
{[0]} & {[G]_{i 2}^{2}} \\
{[0]} & {[G]_{22}^{2}}
\end{array}\right]\left\{\begin{array}{l}
\{P\}^{1} \\
\{P\}^{2}
\end{array}\right\}
$$

Ou de forma simplificada:

$$
[A]\{X\}=[C]\{D\}
$$

Onde $[C]\{D\}$ é conhecido é podendo ser simplificado tornando o sistema assim:

$$
[A]\{X\}=\{B\}
$$

Onde o vetor $\{\mathrm{X}\}$ é o vetor de incógnitas. Portanto por se tratar de um sistema linear de equações, basta empregar uma rotina que seja capaz de resolver sistemas lineares 
esparsos. No programa desenvolvido foi empregada a rotina de Gauss-Jordan com pivoteamento completo.

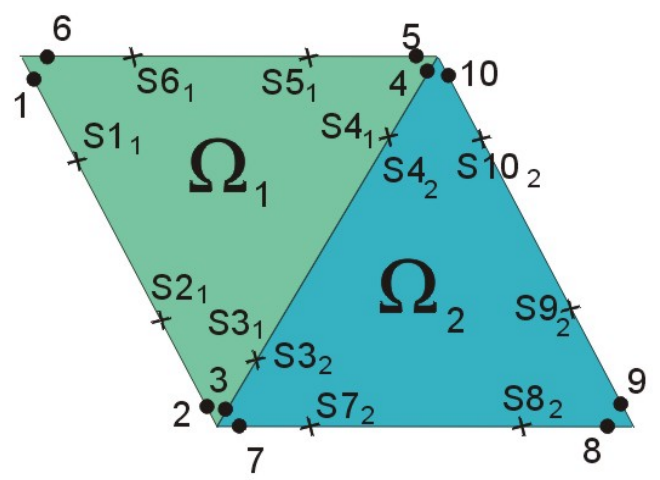

Figura 38 - Geometria e discretização do exemplo.

Observando as matrizes do problema de um problema composto por duas subregiões como é descrito na figura acima, se os sistemas fossem montados de forma independente para cada uma das sub-regiões, ter-se-ia respectivamente para o domínio 1 e 2 :

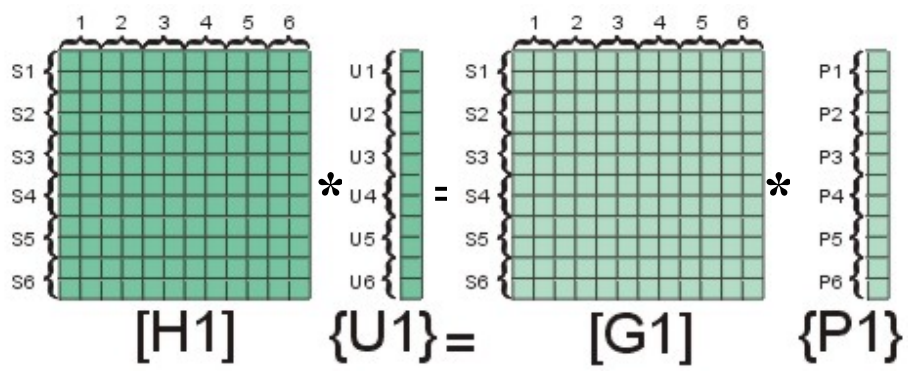

Figura 39 - Sistema de equações do domínio 1.

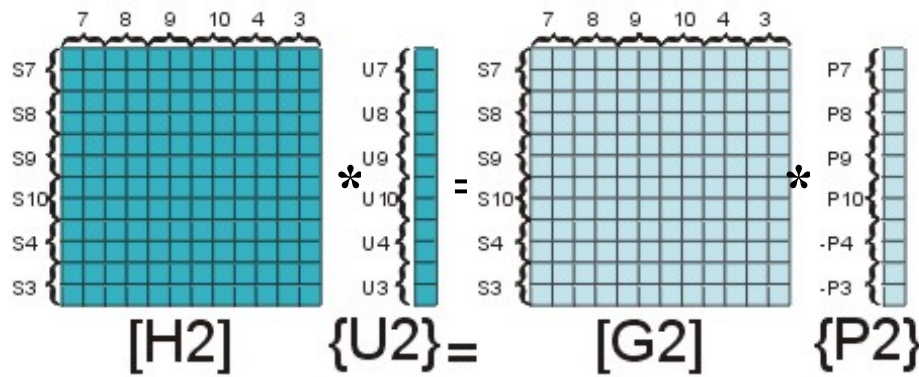

Figura 40 - Sistema de equações do domínio 2.

Logicamente tanto em um quanto o outro sistema não podem ser resolvidos, pois existem mais incógnitas do que equações, já que nos nós da interface entre as duas subregiões tanto o deslocamento quanto à força de contorno são desconhecidas. Este problema só será resolvido quando as condições de equilíbrio forem impostas. Antes disto é 
interessante aplicar as condições de contorno, pois tal procedimento se for aplicado antes de se aplicar às condições de equilíbrio são executados da mesma forma como se faz quando se trata de apenas um domínio.

Os dois sistemas podem ser unidos em um só ficando da seguinte forma:
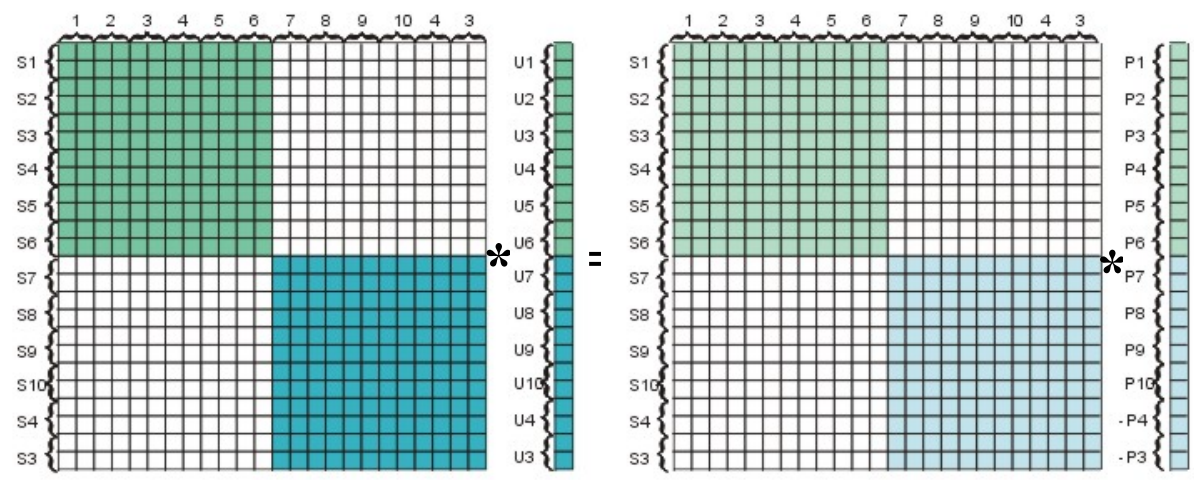

Figura 41 - Junção dos dois sistemas de equações num só.

Aplicando as condições de equilíbrio nos nós que interligam os dois domínios temse:
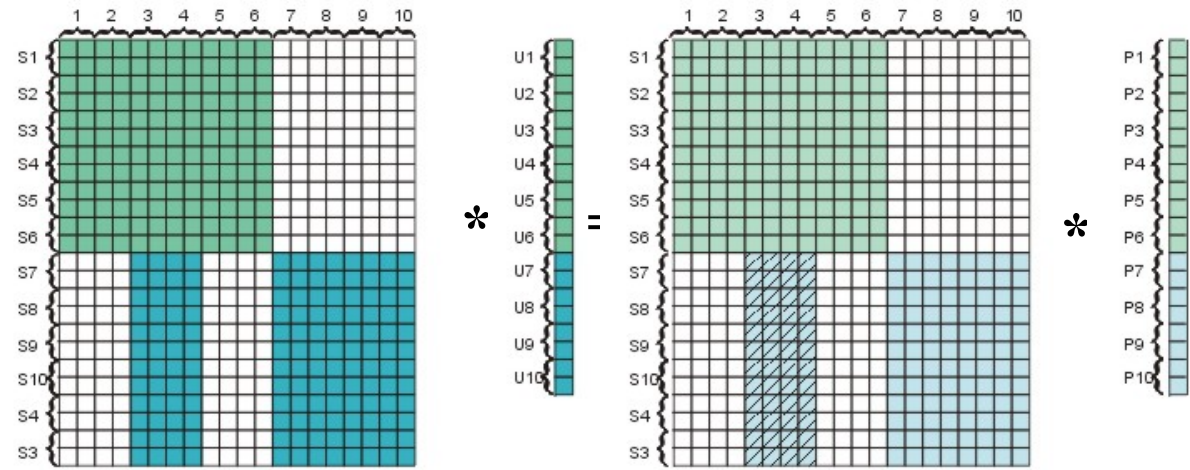

Figura 42 - Aplicação das condições de equilíbrio.

$\mathrm{Na}$ figura acima é importante salientar que a região hachurada representa a região que tem o sinal invertido por causa das condições de equilíbrio.

Para que se possa resolver o sistema de equações, deve-se ainda re-arranjar o sistema deixando-o da seguinte forma: 

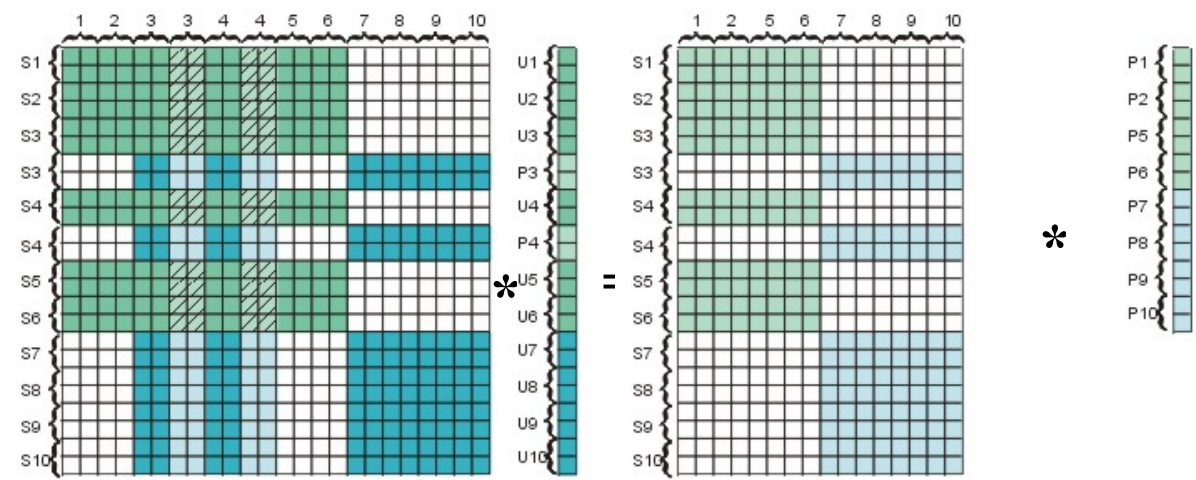

Figura 43 - Rearranjo do sistema de equações.

Lembrando que as regiões hachuradas são as regiões que tem sinal invertido, agora devido ao re-arranjo das equações, inclusive aquelas regiões que estavam hachuradas na figura anterior agora não estão mais, pois novamente foi trocado o sinal desta região cancelando a troca anterior. Este sistema apresentado ilustra a eq (6.8) que agora pode ser transformado na (6.9) e resolvido.

\section{2 - CONDENSAÇÃo DAS INCÓGNITAS DO CONTORNO DO ENRIJECEDOR}

Por tratar-se de sub-regiões finas, pode-se sem grandes perdas aproximar as variáveis de deslocamento normais e tangenciais ao logo da seção transversal do enrijecedor em deslocamentos da linha média e derivadas parciais de deslocamentos, já as forças de superfície podem ser substituídas pelo carregamento que atua sobre uma viga equivalente ao enrijecedor (representada apenas pela linha média).

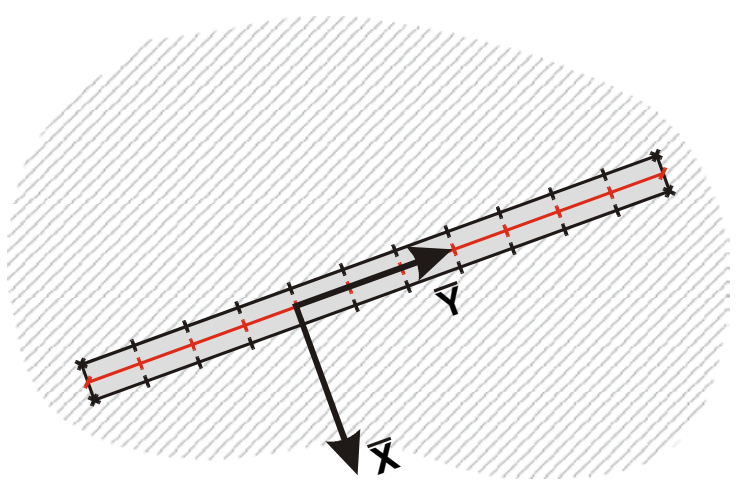

Figura 44 - Enrijecedor com seus eixos locais $\bar{x}$ e $\bar{y}$. 
Segundo os eixos apresentados, para uma aproximação linear em deslocamentos tem-se:

$\mathrm{u}=\mathrm{f}(\overline{\mathrm{x}})=\mathrm{a} \overline{\mathrm{x}}+\mathrm{b}$

Sendo as derivadas com respeito a $\overline{\mathrm{X}}$ descritas por:

$\frac{\partial \mathrm{u}_{\overline{\mathrm{x}}}}{\partial \overline{\mathrm{x}}}=\theta_{\overline{\mathrm{x}}}^{\mathrm{m}} \quad$ e $\quad \frac{\partial \mathrm{u}_{\overline{\mathrm{y}}}}{\partial \overline{\mathrm{x}}}=\theta_{\overline{\mathrm{y}}}^{\mathrm{m}}$

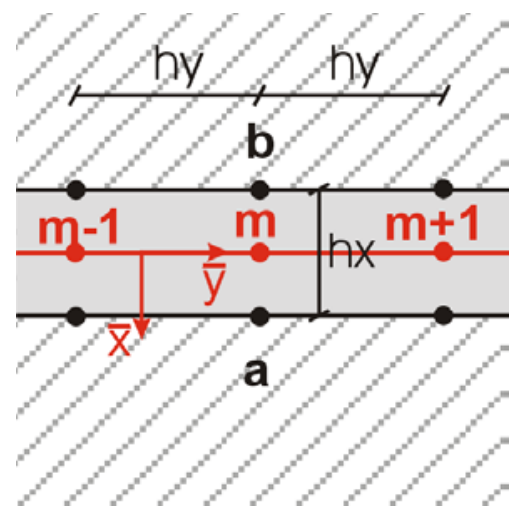

Figura 45 - Nós centrais que recebem as contribuições da integração no contorno.

Lenvando-se em conta a figura 45 pode-se escrever as seguintes equações:

$u_{x}^{a}=u_{x}^{m}+\theta_{x}^{m} \frac{h_{x}}{2}$

$\mathrm{u}_{\mathrm{y}}^{\mathrm{a}}=\mathrm{u}_{\mathrm{y}}^{\mathrm{m}}+\theta_{\mathrm{y}}^{\mathrm{m}} \frac{\mathrm{h}_{\mathrm{x}}}{2}$

$u_{x}^{b}=u_{x}^{m}-\theta_{x}^{m} \frac{h_{x}}{2}$

$\mathrm{u}_{\mathrm{y}}^{\mathrm{b}}=\mathrm{u}_{\mathrm{y}}^{\mathrm{m}}-\theta_{\mathrm{y}}^{\mathrm{m}} \frac{\mathrm{h}_{\mathrm{x}}}{2}$ 
Através das equações (6.12) a (6.15) pode-se substituir as incógnitas $u_{\mathrm{x}}^{\mathrm{a}}, \mathrm{u}_{\mathrm{y}}^{\mathrm{a}}, \mathrm{u}_{\mathrm{x}}^{\mathrm{b}}$ e $\mathrm{u}_{\mathrm{y}}^{\mathrm{b}}$ do contorno pelas incógnitas $\mathrm{u}_{\mathrm{x}}^{\mathrm{m}}, \mathrm{u}_{\mathrm{y}}^{\mathrm{m}}, \theta_{\mathrm{x}}^{\mathrm{m}}$ e $\theta_{\mathrm{y}}^{\mathrm{m}}$ da linha central do enrijecedor como é apresentado na figura 46.

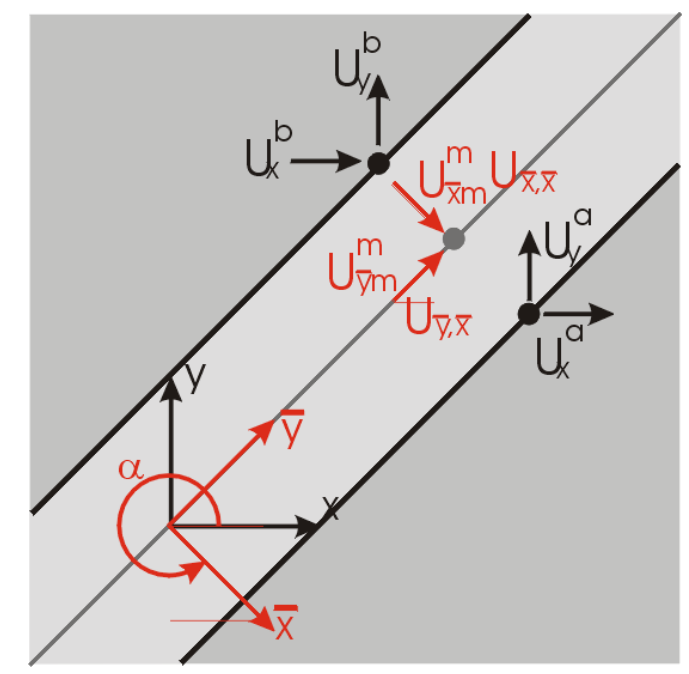

Figura 46 - Transformações das incógnitas de deslocamento do contorno em incógnitas centrais.

De forma semelhante para as forças pode-se ter:

$\mathrm{p}_{\mathrm{x}}^{\mathrm{a}}=\mathrm{p}_{\mathrm{x}}^{\mathrm{m}}+\frac{\Delta \mathrm{p}_{\mathrm{x}}}{2}$

$\mathrm{p}_{\mathrm{y}}^{\mathrm{a}}=\mathrm{p}_{\mathrm{y}}^{\mathrm{m}}+\frac{\Delta \mathrm{p}_{\mathrm{y}}}{2}$

$p_{x}^{b}=p_{x}^{m}-\frac{\Delta p_{x}}{2}$

$\mathrm{p}_{\mathrm{y}}^{\mathrm{b}}=\mathrm{p}_{\mathrm{y}}^{\mathrm{m}}-\frac{\Delta \mathrm{p}_{\mathrm{y}}}{2}$

Sendo assim, através das equações (6.12) a (6.15) pode-se substituir as incógnitas $\mathrm{p}_{\mathrm{x}}^{\mathrm{a}}, \mathrm{p}_{\mathrm{y}}^{\mathrm{a}}, \mathrm{p}_{\mathrm{x}}^{\mathrm{b}}$ e $\mathrm{p}_{\mathrm{y}}^{\mathrm{b}}$ do contorno pelas incógnitas $\mathrm{p}_{\mathrm{x}}^{\mathrm{m}}, \mathrm{p}_{\mathrm{y}}^{\mathrm{m}}, \Delta \mathrm{p}_{\mathrm{x}}$ e $\Delta \mathrm{p}_{\mathrm{y}}$ da linha central do enrijecedor. Desta forma as incógnitas que seriam descritas na linha central seriam a forças 
médias e não as forças resultantes no enrijecedor. Mas pode-se optar por escrever as incógnicas da seguinte forma:

$\mathrm{p}_{\mathrm{x}}^{\mathrm{a}}=\frac{\mathrm{p}_{\mathrm{xr}}^{\mathrm{m}}}{2}+\frac{\Delta \mathrm{p}_{\mathrm{x}}}{2}$

$\mathrm{p}_{\mathrm{y}}^{\mathrm{a}}=\frac{\mathrm{p}_{\mathrm{yr}}^{\mathrm{m}}}{2}+\frac{\mathrm{M}_{\mathrm{r}}}{\mathrm{h}_{\mathrm{x}}}$

$\mathrm{p}_{\mathrm{x}}^{\mathrm{b}}=\frac{\mathrm{p}_{\mathrm{xr}}^{\mathrm{m}}}{2}-\frac{\Delta \mathrm{p}_{\mathrm{x}}}{2}$

$p_{\mathrm{y}}^{\mathrm{b}}=\frac{\mathrm{p}_{\mathrm{yr}}^{\mathrm{m}}}{2}-\frac{\mathrm{M}_{\mathrm{r}}}{\mathrm{h}_{\mathrm{x}}}$

Ficando assim com as incógnitas do contorno $\mathrm{p}_{\mathrm{x}}^{\mathrm{a}}, \mathrm{p}_{\mathrm{y}}^{\mathrm{a}}, \mathrm{p}_{\mathrm{x}}^{\mathrm{b}}$ e $\mathrm{p}_{\mathrm{y}}^{\mathrm{b}}$ em função das incógnitas centrais $\mathrm{p}_{\mathrm{xr}}^{\mathrm{m}}, \mathrm{p}_{\mathrm{yr}}^{\mathrm{m}}, \mathrm{M}_{\mathrm{r}} \mathrm{e} \Delta \mathrm{p}_{\mathrm{x}}$, sendo $\mathrm{p}_{\mathrm{xr}}^{\mathrm{m}}$ a carga resultante em $\mathrm{x}$ atuante no enrijecedor, $\mathrm{p}_{\mathrm{yr}}^{\mathrm{m}}$ também a carga resultante agora em y, $\mathrm{M}_{\mathrm{r}}$ o momento fletor resultante que atua no enrijecedor e $\Delta \mathrm{p}_{\mathrm{x}}$ simplesmente a variação de cargas normais a seção. Como é apresentado na figura 47.

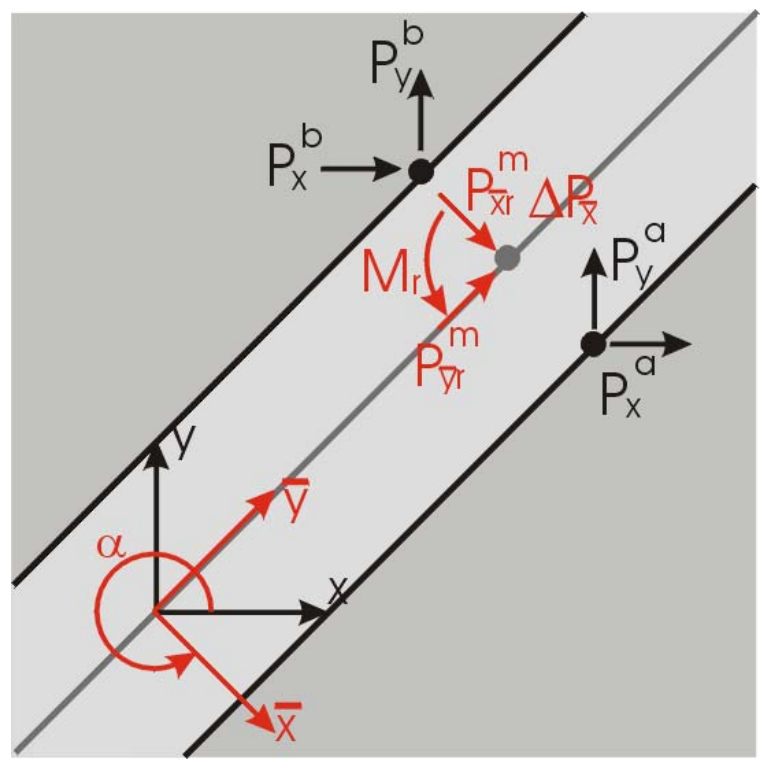

Figura 47 - Transformações das incógnitas de força do contorno em esforças resultantes do enrijecedor. 
Tanto as incógnitas $\Delta \mathrm{p}_{\mathrm{x}}$ e $\Delta \mathrm{p}_{\mathrm{y}}$, como $\mathrm{M}_{\mathrm{r}}$ e $\Delta \mathrm{p}_{\mathrm{x}}$, poderão ainda serem escritas com auxílio das equações da lei Hooke, relação deformação-deslocamento e por diferenças finitas em função das variáveis $\mathrm{u}_{\mathrm{x}}^{\mathrm{m}}, \mathrm{u}_{\mathrm{y}}^{\mathrm{m}}, \theta_{\mathrm{x}}^{\mathrm{m}}$ e $\theta_{\mathrm{y}}^{\mathrm{m}}$, podendo desta forma reduzir o número de incógnitas sem grandes conseqüências para os resultados em caso de enrijecedores de pequena espessura.

\section{3- EXEMPLOS DE APLICAÇÃO}

Para demonstrarmos emprego do modelo acima descrito são apresentados três exemplos:

No primeiro exemplo é simulado um enrijecedor inserido transversalmente a uma viga simplesmente tracionada. Para este exemplo são apresentados os esforços atuantes no enrijecedores tanto da forma convencional (por sub-região) como da forma condensada. Deseja-se com isso deixar mais claro que resultados são obtidos quando se utiliza a técnica de condensação das variáveis.

No segundo exemplo, de forma semelhante ao primeiro é feita apenas uma comparação dos resultados obtidos na forma convencional e na forma condensada para uma viga engastada com um enrijecedor na parte inferior da mesma submetida à uma carga distribuída em sua face superior.

No terceiro exemplo são estudados diversos casos de reforço de um tirante com enrijecedores, onde busca-se mostrar a eficiência da técnica de condensação para espessuras muito pequenas de enrijecedores.

\subsection{1 - Exemplos 01: Chapa enrijecida transversalmente submetida a uma tração simples.}

Como foi citado anteriormente neste exemplo é apresentado o resultado dos esforços atuantes num enrijecedor inserido transversalmente a uma chapa simplesmente tracionada. Apesar de que o mais comum seria colocar o enrijecedor na direção longitudinal (na direção 
do carregamento), foi colocado no sentido transversal ao carregamento apenas para mostrar a diferença existente entre os resultado obtidos na técnica de sub-região e a condensação. Deixando claro assim que na condensação, os deslocamentos apresentados são os deslocamentos da linha média do enrijecedor e as derivadas dos deslocamentos com respeito ao eixo normal do elemento. Já as forças de contorno são condensadas em forças resultantes atuantes do enrijecedor, momento fletor atuante no mesmo e variação das forças normais ao enrijecedor.

Os dados geométricos e físicos do problema em questão estão apresentados na figura 48.

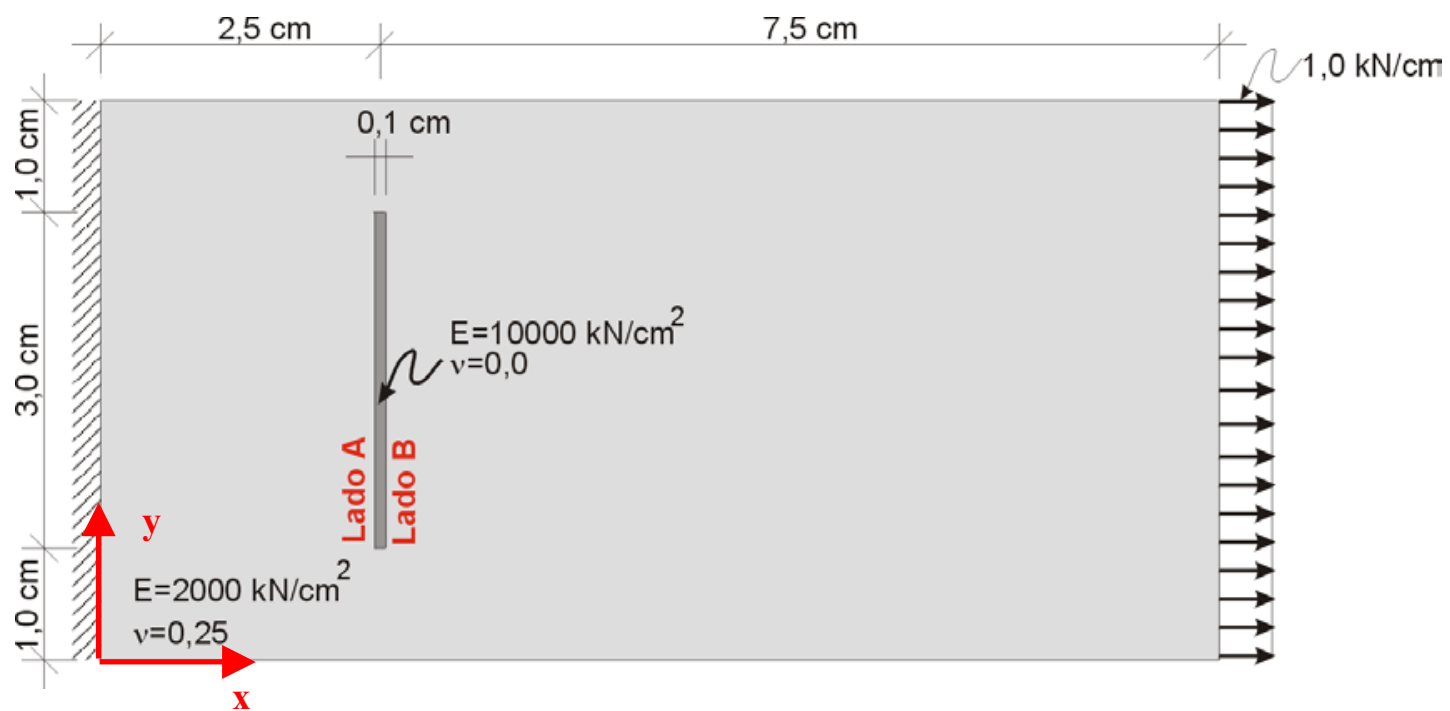

Figura 48 - Dados do problema 1.

Os resultados apresentados foram obtidos com a seguinte discretização (Figura 49):

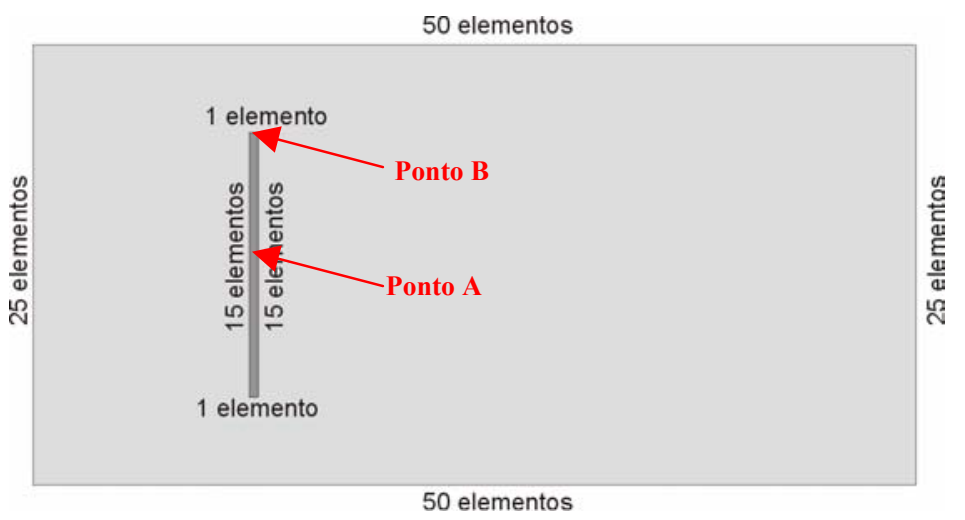

Figura 49 - Discretização do problema 1. 
Os Gráficos 12 e 13 apresentam uma comparação entre os deslocamentos obtidos por sub-região nas interfaces do enrijecedor e os deslocamentos obtidos na seção media através da condensação.

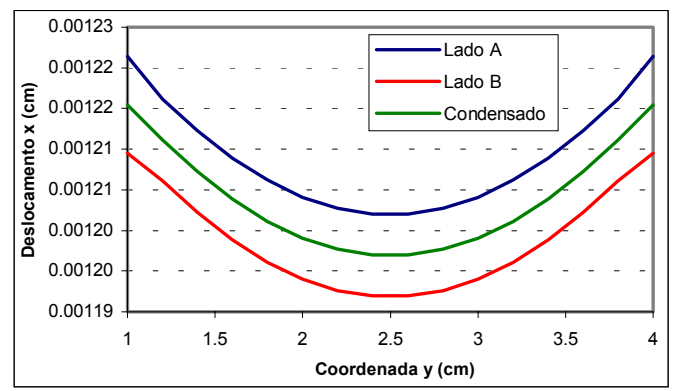

Gráfico 12 - Deslocamentos na direção X do enrijecedor.

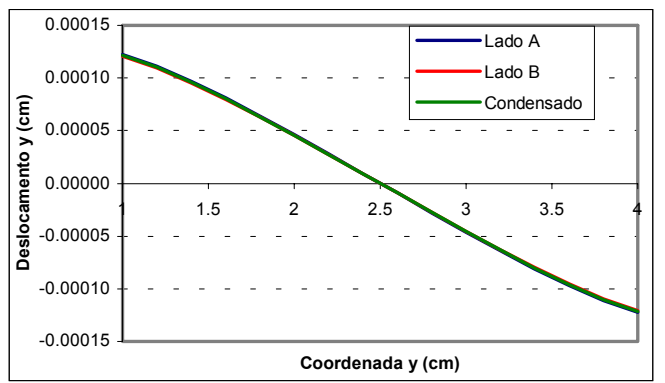

Gráfico 13 - Deslocamentos na direção Y do enrijecedor.

E nos gráficos 14 e 15 são comparadas os esforças na interface do enrijecedor (técnica de sub-região) com os esforços resultantes (por condensação).

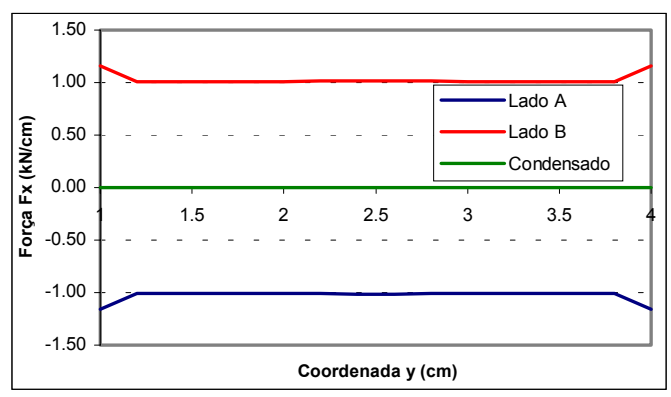

Gráfico 14 - Esforços na direção X atuantes no enrijecedor.

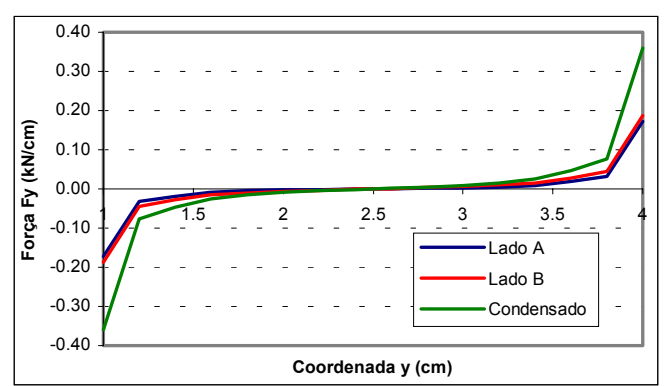

Gráfico 15 - Esforços na direção Y atuantes no enrijecedor.

Como conseqüência da maneira com que foram condensadas as variáveis de deslocamento, surgem como respostas para o problema as derivadas parciais de desclocamento com respeito ao eixo $\mathrm{X}$ (eixo local norma ao eixo da linha central do enrijecedor). Sendo os resultados apresentados a seguir nos gráficos 16 e 17 . 


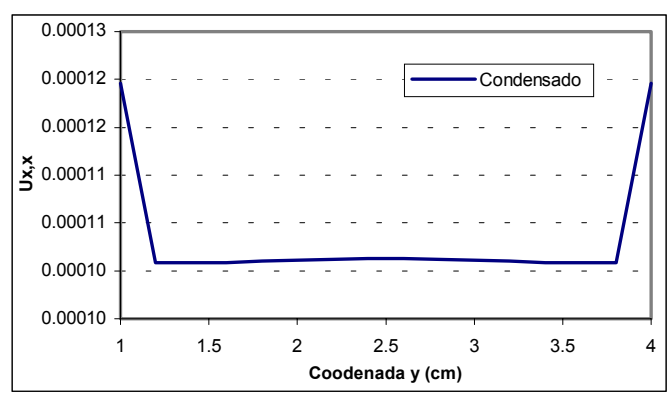

Gráfico 16 - Gráfico de $\partial \mathrm{u}_{\mathrm{x}} / \partial \mathrm{x}$ no enrijecedor obtido na condensação.

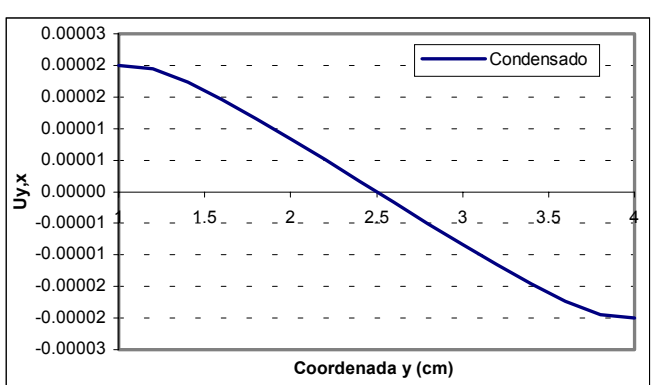

Gráfico 17 - Gráfico de $\partial \mathrm{u}_{\mathrm{y}} / \partial \mathrm{x}$ no enrijecedor obtido na condensação.

E por fim, os diagramas de Momento Fletor e variação da força Normal obtidos na condensação das variáveis de força para o enrijecedor são apresentadas nos gráficos 18 e 19.

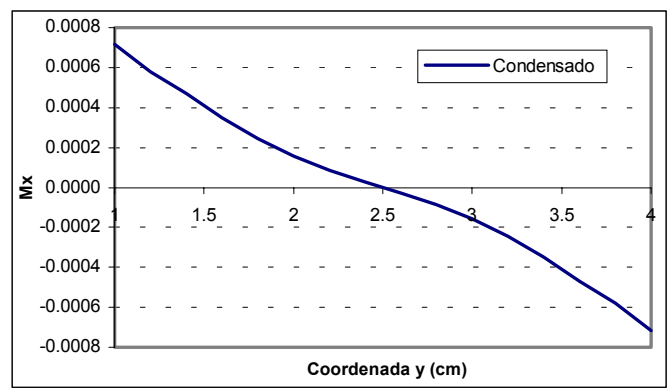

Gráfico 18 - Gráfico do momento fletor atuante no enrijecedor.

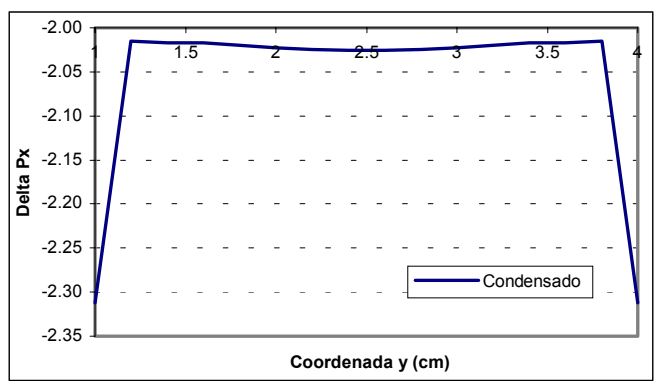

Gráfico 19 - Gráfico da variação da força normal entra as interfacces do enrijecedor.

$\mathrm{Na}$ tabela 04 pode-se observar uma comparação entre os resultado encontrados para a técnica de sub-região e a de condensação com o mesmo problema simulado com elementos finitos para uma malha bem discretizada (2700 Elementos Shel193) no programa Ansys. Como se pode observar os valeres encontrados com os elementos finitos é semelhante aos encontrados com o MEC tanto por sub-região quanto por condensação.

\begin{tabular}{ccc}
\hline Método de cálculo & $\mathbf{U}_{\mathbf{x}}$ do Ponto A & $\mathbf{U}_{\mathbf{x}}$ do Ponto B \\
\hline Sub-região & 0.001197 & 0.001215 \\
Condensação & 0.001197 & 0.001215 \\
SHELL 93 (ANSYS) & 0,001221 & 0.001238 \\
\hline
\end{tabular}

Tabela 04 - Deslocamentos em x no meio e na ponta do enrijecedor. 


\subsection{2 - Exemplos 02: Viga engastada submetida a uma carga distribuída na face superior reforçada com um enrijecedor em sua face inferior.}

Assim como o exemplo anterior este exemplo busca apenas mostrar as diferenças entre os resultados que se obtém através da técnica de sub-região e a de condensação.

Os dados geométricos e físicos do problema bem como suas condições de contorno são apresentadas na figura 50, já a discretização adotada pode ser observada na figura 51.

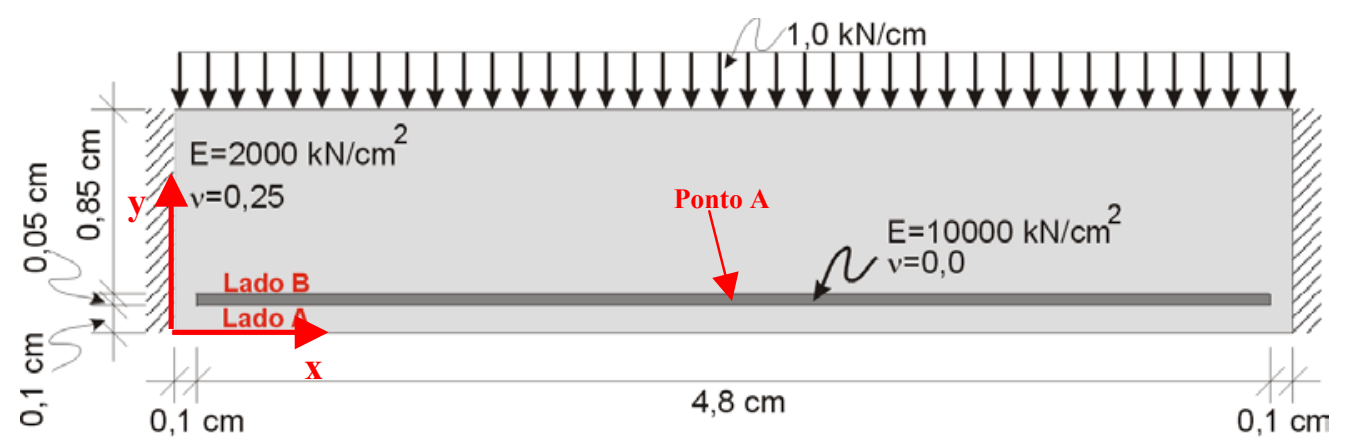

Figura 50 - Dados físicos, geométricos e condições de contorno do exemplo 2.

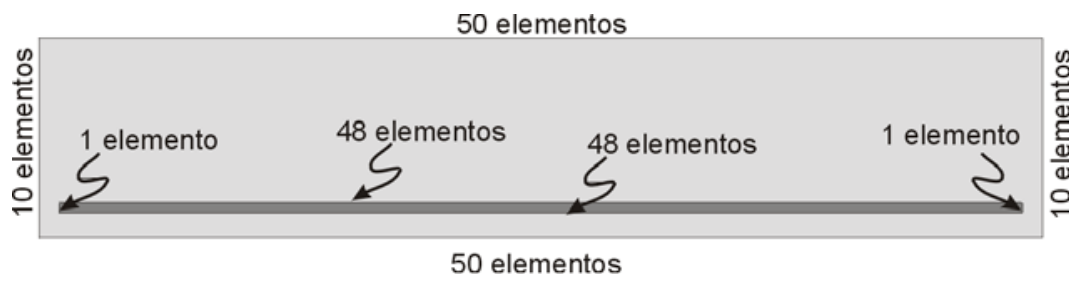

Figura 51 - Discretização empregada no exemplo 2.

Para o problema descrito anteriormente, os resultados dispostos de forma semelhante ao exemplo anterior podem ser observados nos gráficos 20 e 21 (deslocamentos do enrijecedor), 22 e 23, esforços atuantes no enrijecedor, 24 e 25 derivadas dos deslocamentos com respeito ao eixo x local, momento fletor e variação do esforço normal nos gráficos 26 e 27.

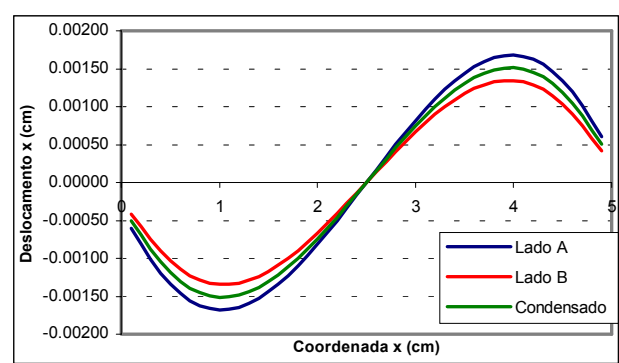

Gráfico 20 - Deslocamentos na direção X do enrijecedor.

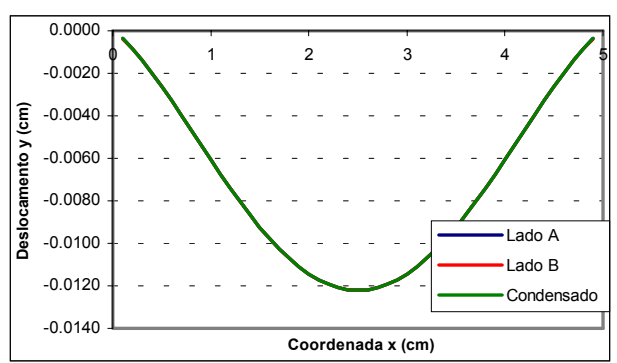

Gráfico 21 - Deslocamentos na direção Y do enrijecedor. 


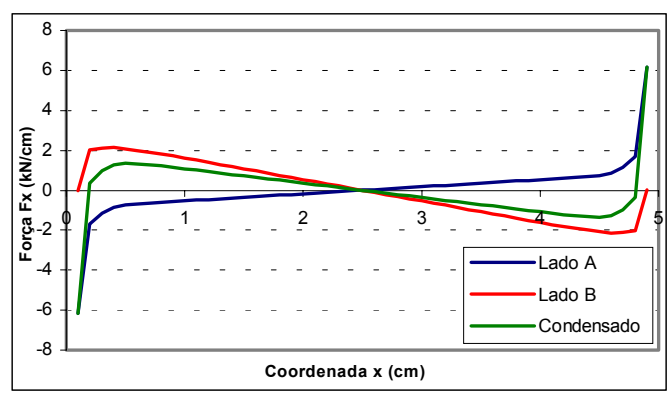

Gráfico 22 - Esforços na direção $\mathrm{X}$ atuantes no enrijecedor.

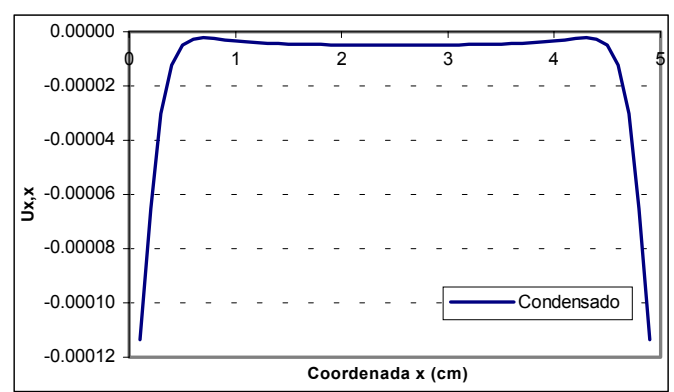

Gráfico 24 - Gráfico de $\partial \mathrm{u}_{\mathrm{x}} / \partial \mathrm{x}$ no enrijecedor obtido na condensação.

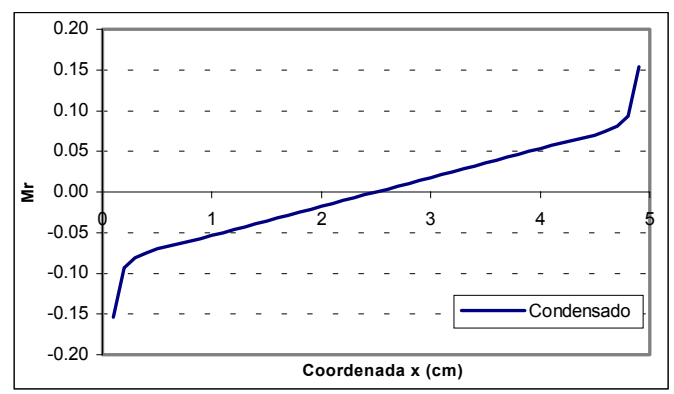

Gráfico 26 - Gráfico do momento fletor atuante no enrijecedor.

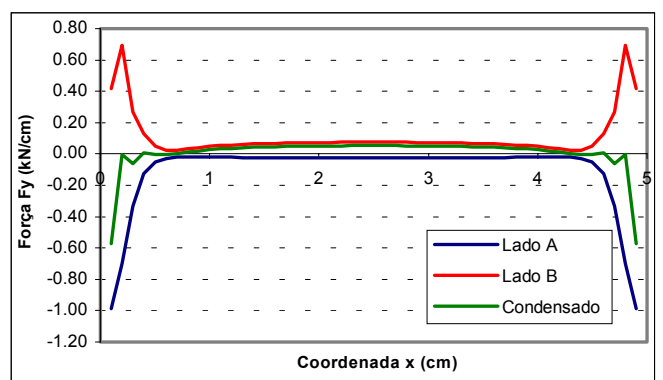

Gráfico 23 - Esforços na direção Y atuantes no enrijecedor.

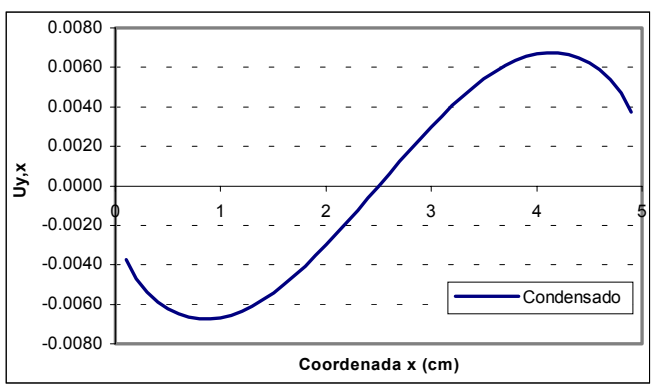

Gráfico 25 - Gráfico de $\partial \mathrm{u}_{\mathrm{y}} / \partial \mathrm{x}$ no enrijecedor obtido na condensação.

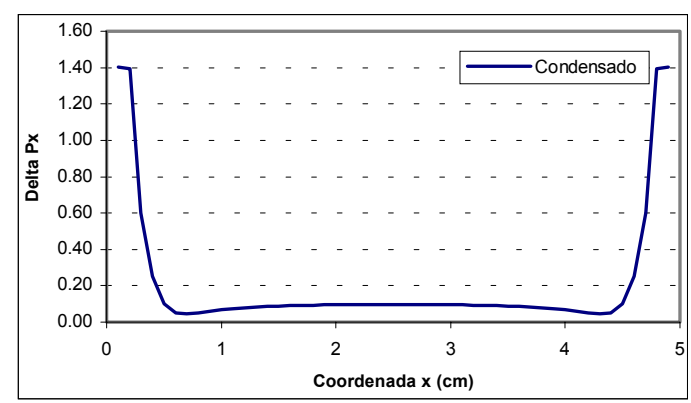

Gráfico 27 - Gráfico da variação da força normal entra as interfacces do enrijecedor.

A Tabela 05 compara o deslocamento vertical da Ponto A localizado no centro do enrigecedor. São comparados os resultados da condensação, sub-região e elementos finitos sendo este último simulado com auxílio do programa Ansys com uma malha composta por 2500 elementos finitos Shell 93. 


\begin{tabular}{cc}
\hline Método de cálculo & Deslocamento $\mathbf{Y}(\mathbf{c m})$ \\
\hline Sub-região & $-0,012211$ \\
Condensação & $-0,012211$ \\
SHELL 93 (ANSYS) & $-0,012368$ \\
\hline
\end{tabular}

Tabela 05 - Deslocamentos em y do ponto A.

Como se pode observar o resultado apresentado pelo Ansys é semelhante ao encontrado pelo método dos elementos de contorno tanto por sub-região quanto por condensação.

\subsection{3 - Exemplos 03: Tirante reforçado com enrijecedores.}

Neste exemplo buscou-se testar do programa para enrijecedores extremamente finos. Para tanto, o tirante apresentado na figura 52, foi simulado várias vezes variando-se a espessura é o módulo de elasticidade dos enrijecedores, várias discretizações fora estudadas também, mas os resultados apresentados são sempre para a discretização apresentada na figura 53. Os gráficos à serem descritos a seguir sempre se referem ao enrijecedor cuja coordenada x é menor (o mais inferior da figura 52). Nos diagramas quando se refere ao lado "A" do enrijecedor se refere ao lado inferior e lado "B" o lado superior do enrijecedor em questão.

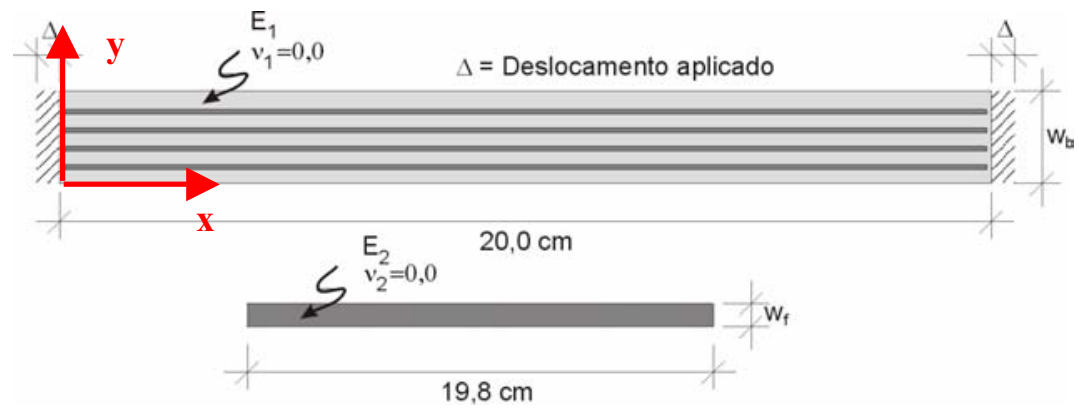

Figura 52 - Dimensões e condições de contorno simuladas no exemplo 3.

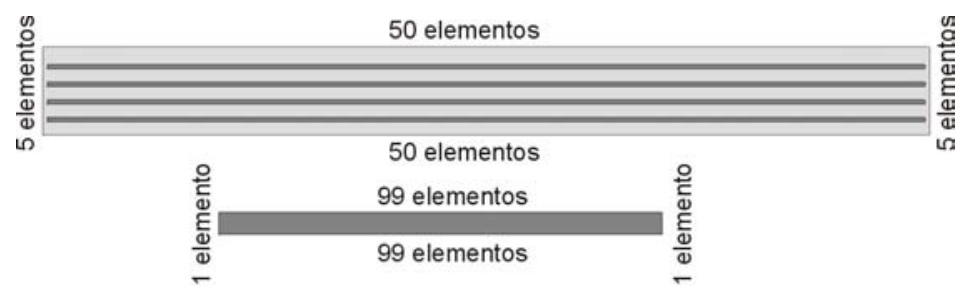

Figura 53 - Discretização cujos resultados são apresentados no exemplo 3. 
Separou-se alguns casos simulados em dois casos para que os resultados fossem apresentados. Seguiu-se a seguinte relação de proporcionalidade entre as variáveis físicas do problema:

$$
4 \mathrm{E}_{2} \mathrm{~A}_{2}=\alpha \mathrm{E}_{1} \mathrm{~A}_{1}
$$

Onde:

$\mathrm{E}_{2}$ - Módulo de elasticidade dos enrijecedores.

$\mathrm{A}_{2}$ - Área da seção transversal de cada enrijecedor.

$\mathrm{E}_{1}$ - Módulo de elasticidade do tirante.

$\mathrm{A}_{1}$ - Área da seção transversal do tirante.

$\alpha$ - Constante de proporcionalidade.

Para todos os resultados aqui apresentados, $\mathrm{E}_{1}$ era constante e igual à $2000 \mathrm{kN} / \mathrm{cm}^{2}$. $\mathrm{A}_{2}$ e $\mathrm{A}_{2}$ podem ser calculados em função das variáveis $\mathrm{w}_{\mathrm{f}}$ e $\mathrm{w}_{\mathrm{b}}$ adotadas em cada caso. $\mathrm{A}_{2}$ pode ser calculado pela equação (6.24) a partir do parâmetro $\alpha$ e da relação $\mathrm{w}_{\mathrm{f}} / \mathrm{w}_{\mathrm{b}}$ adotados em cada caso.

No primeiro bloco de resultados é avaliada somente a influência da variação da taxa de armadura no esforço de cisalhamento. Para tanto é adotada uma relação $\mathrm{w}_{\mathrm{f}} / \mathrm{w}_{\mathrm{b}}$ igual a 0,05 , o parâmetro $\alpha$ varia de 1 a 4 , um quinto caso ainda é analizado, onde o parâmetro $\alpha$ seria igual à 0.19047619047619 que é justamente o caso em que a relação $E_{2} / E_{1}$ é igual a 1 (caso em que não há reforço algum).

O esforço cortante apresentado para cada um dos casos anteriores pode ser observado nos gráficos 28 e 29, o gráfico 28 mostra as forças cortantes para as duas faces do enrijecedor simulado com a técnica de sub-região, já no gráfico 29 é mostrada as forças cortantes resultantes que atuam no enrijecedor simuladas com condensação de variáveis. 


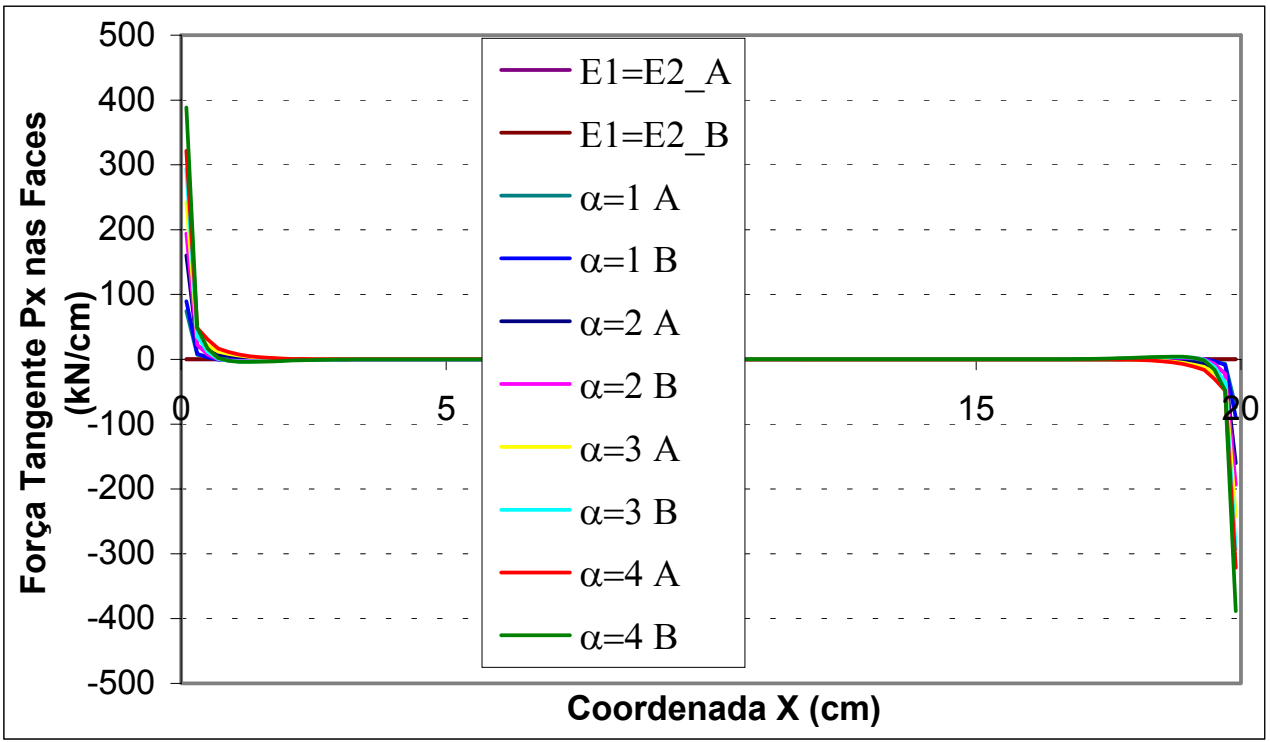

Gráfico 28 - Força cortante na duas faces do enrijecedor número 1 simulado com a técnica de sub-região.

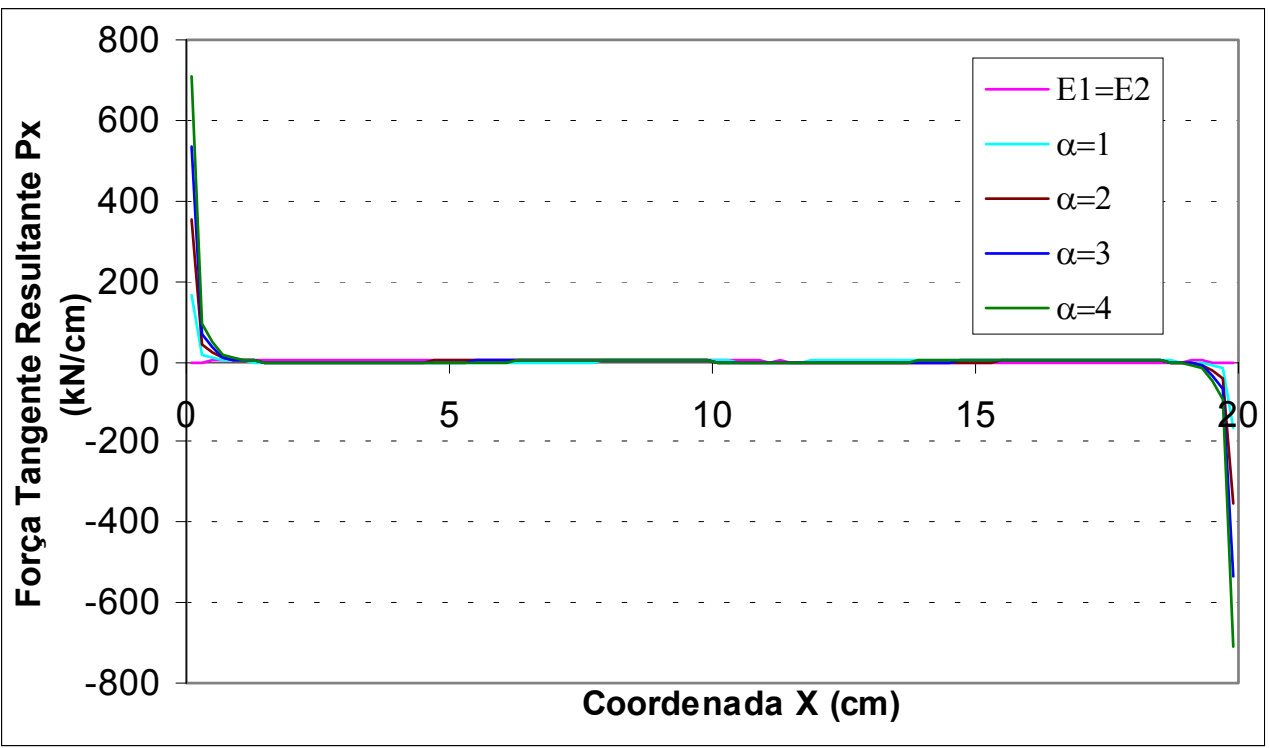

Gráfico 29 - Força cortante resultante que atua no enrijecedor número 1 simulado com a condensação de variáveis.

Pode-se facilmente notar que os resultados apresentados pela condensação têm para este caso valores duas vezes maior ao apresentados pela técnica de sub-região, isto se deve ao fato de que para a condensação o resultado encontrado é a resultante das forças cortantes que é justamente a soma das duas cortantes apresentadas nas duas faces. 
O segundo grupo de simulações avalia o comportamento das duas técnicas para espessuras de enrijecedores muito estreitos. Para isto os resultados apresentados tratam-se dos obtidos através da simulação dos seguintes casos: $\alpha$ é constante em todos os casos e igual a $1, \mathrm{w}_{\mathrm{f}} / \mathrm{w}_{\mathrm{b}}$ com os seguintes valores para cada caso: $5 * 10^{-2}, 5 * 10^{-3}, 5 * 10^{-4}$ e $5 * 10^{-5}$. Seguindo-se sempre a relação de proporcionalidade descrita na equação (6.24).

São apresentados nos gráficos 30 e 31 os deslocamentos no eixo $X$ para o enrijecedor número 1 simulado em todos os casos descritos acima, sendo o gráfico 30 para sub-região e o gráfico 31 para condensação.

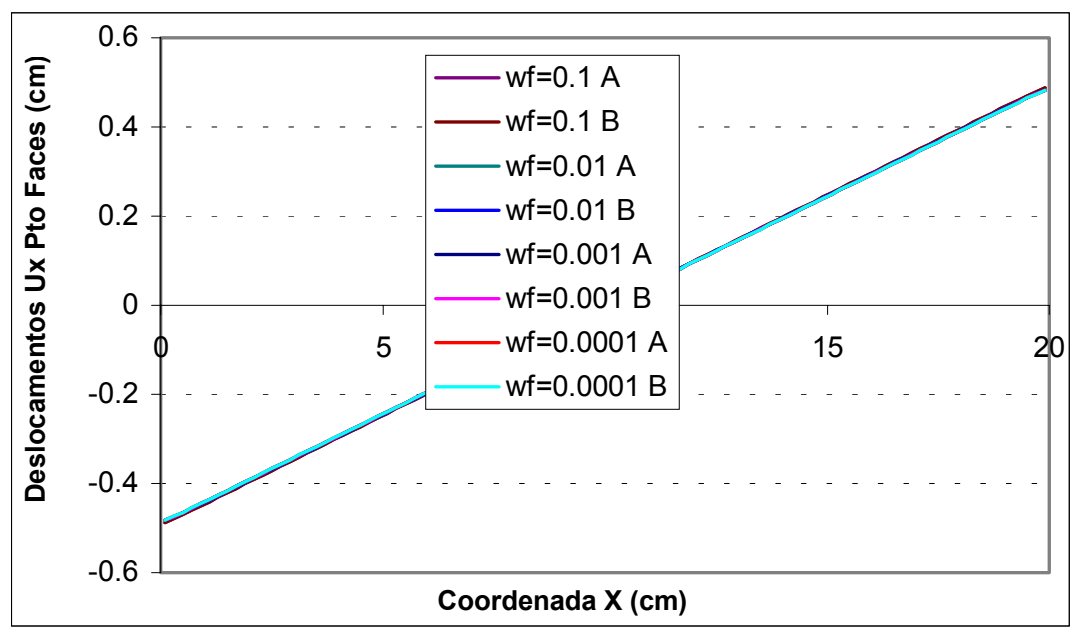

Gráfico 30 - Deslocamento no eixo x das duas faces do enrijecedor número 1 simulado com a técnica de sub-região.

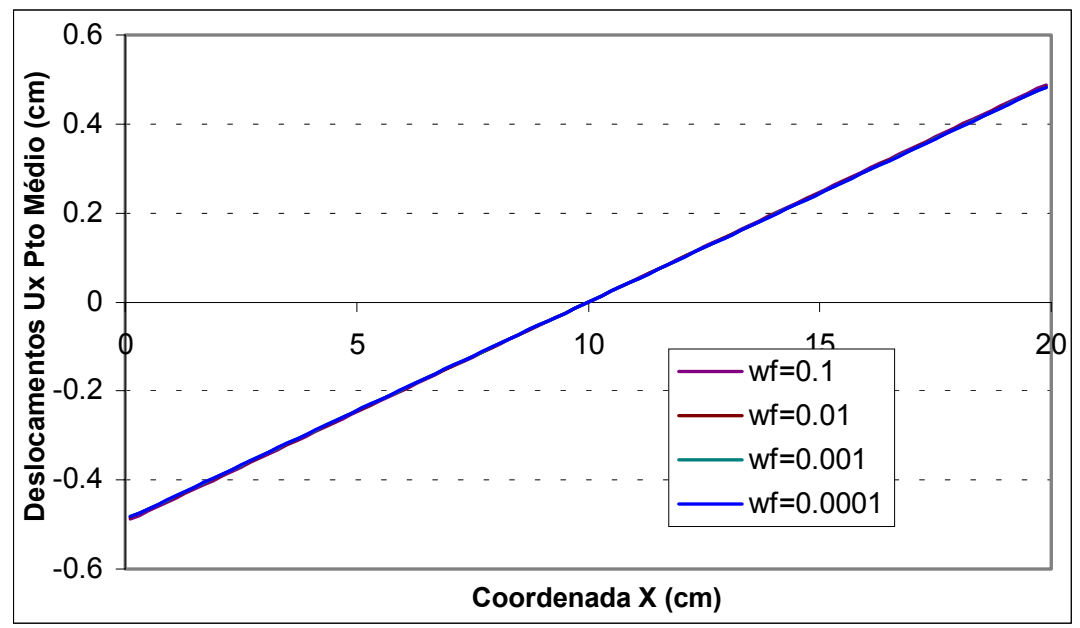

Gráfico 31 - Deslocamento médio no eixo x do enrijecedor número 1 simulado com a condensação de variáveis. 
Já nos gráficos 32 e 33 a força cortante é apresentada sendo o gráfico de 32 com subregião e o 33 com condensação.

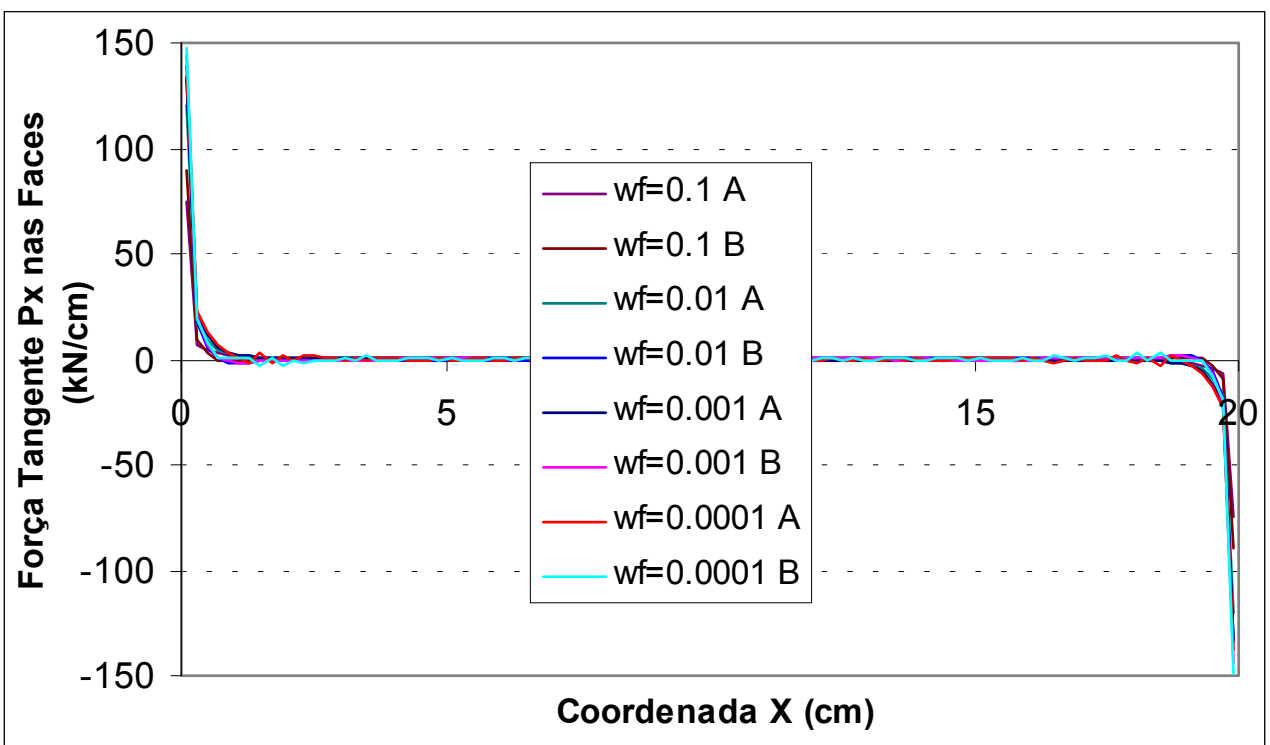

Gráfico 32 - Força cortante nas duas faces do enrijecedor número 1 simulado com a técnica de sub-região.

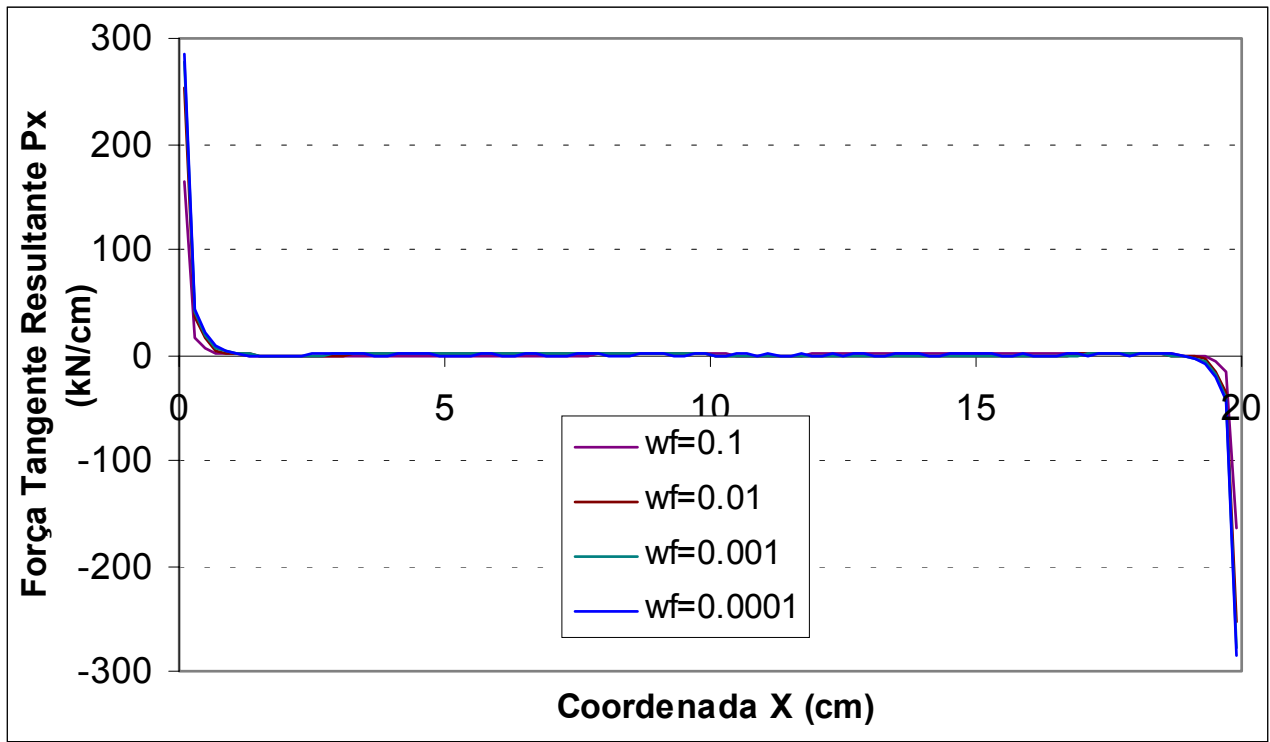

Gráfico 33 - Força cortante resultante do enrijecedor número 1 simulado com a condensação de variáveis.

Se observarmos o último caso em que a espessura $\mathrm{w}_{\mathrm{f}}$ adotada é de $1 * 10^{-4}$, logo, relação $\mathrm{w}_{\mathrm{f}} / \mathrm{w}_{\mathrm{b}}$ igual a $5 * 10^{-5}$. Para a condensação há uma regularização dos resultados 
fazendo com que a perturbação apresentada na técnica de sub-região desaparecesse em espessuras muito diminutas simuladas com a técnica de condensação.

\section{4 - CONCLuSÕes PARCIAIS}

Como esperávamos a técnica de condensação de variáveis para representação de enrijecedores estreitos obtém bons resultados regularizando distorções decorrentes da pequena espessura para resposta de forças de superfície. Contudo não podemos esquecer que a integração analítica para os casos singulares e não singulares também teve sua parcela de contribuição viabilizando a técnica devido à melhoria das integrações. Para problemas não lineares ou de análise inversa essas regularizações são muito importantes para obtenção de bons resultados.

Várias formas de condensação podem ser empregadas, contudo é importante se ter em mente o problema que se está estudando para evitar simplificações que alterem o problema original em que se quer tratar. Apesar de que a forma que foi tratada a condensação das variáveis não contemplar a redução de graus de liberdade do sistema, tal situação pode ser abordada se transformarmos as variáveis referentes ao momento fletor e à variação da força normal nas faces do enrijecedor, em deformações e deslocamentos médios do enrijecedor, aplicando-se para isso a lei de Hooke, relação deformação deslocamento e diferenças finitas. Deve-se, contudo ponderar o fato de que ao aplicarmos diferenças finitas nesta aproximação, isto levaria talvez a uma perda considerável de precisão estragando assim os resultados. 


\section{INTERFACE GRÁFICA}

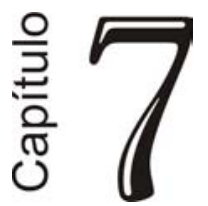

Neste capítulo é apresentadas apenas uma breve revisão bibliográfica sobre computação gráfica, OpenGL e a linguagem de programação Delphi. Recursos que foram amplamente empregados na criação do pré e do pós-processador desenvolvido em linguagem Delphi com sub-rotinas gráficas escritas em OpenGL e gerador de malha em C++.

Com auxílio de alguns algoritmos e de uma interface gráfica desenvolvida em OpenGL utilizando o Delphi como plataforma, criou-se um programa que tem por principal finalidade facilitar e agilizar a geração e análise de exemplos com o método dos elementos de contorno.

Facilidades como geração semi-automática dos elementos de contorno, geração de células de domínio de forma independente e interativamente, podendo ser reajustadas se for o caso, funções que facilitam a definição de nós duplos, geração de sub-regiões, aplicação de condições de contorno, foram desenvolvidas neste programa.

Ainda no pós-processamento dos resultados pode-se observá-los através de uma interface gráfica também desenvolvida em OpenGL com recursos de "zoom" translação, rotação. Recursos como a visualização de isolinhas, posição deformada ou não, configuração da escala de cores também foram desenvolvidos.

\subsection{Pequena Revisão Bibliográfica}

\section{Computação Gráfica}


O primeiro computador a possuir recursos gráficos de visualização de dados numéricos foi o "Whirlwind I" (furacão), desenvolvido pelo MIT. Este equipamento foi desenvolvido, em 1950, com finalidades acadêmicas e também possivelmente militares, pois logo em seguida o comando de defesa aérea dos EUA desenvolveu um sistema de monitoramento e controle de vôos (SAGE - Semi-Automatic Ground Enviroment).

Em 1962, surgiu uma das mais importantes publicações de Computação Gráfica de todos os tempos, a tese do Dr. Ivan Sutherland ("Sketchpad - A Man-Machine Graphical Communication System"), propunha uma forma de interação muito semelhante ao que hoje chamados de interfaces WIMP - Window-Icon-Menu-Pointer.

Esta publicação chamou a atenção das indústrias automobilísticas e aeroespaciais americanas. Os conceitos de estruturação de dados bem como o núcleo da noção de Computação Gráfica interativa levaram a General Motors a desenvolver o precursor dos primeiros programas de C.A.D.

Hoje em dia a computação gráfica pode ser empregada para as mais diversas aplicações como, por exemplo: traçado interativo de gráficos e visualização, editoração eletrônica, CAD - do inglês Computer Aided Design, que quer dizer projeto assistido por computador, simulação e animação, controle/visualização de processos. cartografia e etc.

\section{OpenGL}

OpenGL (Open Graphics Library), como o próprio nome diz, é uma poderosa biblioteca de rotinas gráficas para modelagem 2D e 3D. OpenGL independe da linguagem de programação e do sistema utilizado, mas nem sempre é igual à utilização dessa biblioteca com linguagens e/ou plataformas diferentes, embora as rotinas de exibição de objetos serão sempre iguais.

A OpenGL foi desenvolvida pela Silicon Graphics, que hoje é a maior empresa de Computação Gráfica e Animação. As grandes vantagens de utilizar OpenGL para criar aplicativos gráficos, é que ela se mostra bastante rápida e eficiente na hora de trabalhar com gráficos 3D, sem falar que é uma biblioteca aberta.

Como a tarefa de integrar OpenGL a uma linguagem, nem sempre é trivial, existem diversas ferramentas (bibliotecas) para este fim, especialmente para Delphi e Visual Basic. Estas ferramentas são componentes ou OCXs que encapsulam a OpenGL, e tornam mais fácil a integração com estas linguagens. 
Criada pela Silicon Graphics, trata-se de uma API de funções que permite entre outras coisas criar janelas, ler o teclado e o mouse e criar menus de opções. Existem versões padronizadas da GLUT para DOS, Windows, Linux, MacOS e para a maioria das plataformas UNIX.

A grande vantagem do uso da GLUT é que ela permite o uso de todas as funções gráficas OpenGL e ainda torna padronizado o acesso a características específicas de cada ambiente de janelas.

\section{DELPHI}

O Delphi é um ambiente de desenvolvimento de aplicações, orientado a objeto, que permite desenvolver programas para alguns sistemas operacionais, por enquanto somente da família Windows da Microsoft.

Em 1642, Blaise Pascal um famoso matemático, físico e filósofo francês, projetou a primeira máquina de calcular, isto aos 18 anos, chamada pascalina. Deste matemático surgiu uma maneira de dizer ao microcomputador as tarefas que deveriam ser realizadas, essa maneira era escrever códigos através do $n^{\circ} 0$ e 1 denominados NÚMEROS BINÁRIOS, sendo que 0 (desligado) e 1 (ligado).

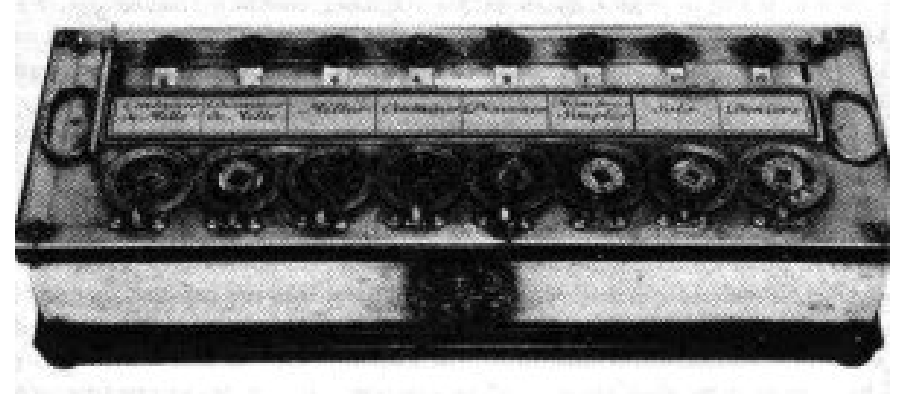

Figura 54 - Pascalina, a maquina de calcular desenvolvida pelo matemático, físico e filósofo francês Blasi Pascal.

Niklaus Wirth cria, em 1971, uma linguagem simbólica (PASCAL) tendo em vista o ensino das técnicas de programação e dá origem à técnica de Programação Estruturada. Sendo o nome da linguagem, "Pascal" uma homenagem a Baise Pascal e a sua máquina a pascalina. 
Deu-se então origem as linguagens de programação, que logicamente teve uma que levou o nome do matemático, denominada PASCAL, apareceram várias linguagens de programação, como ADA, CLIPPER, ASSEMBLER, COBOL, C, C++ , FORTRAN, entre outras e atualmente existem várias linguagens, cada uma com sua característica, como PHYTON, VISUAL BASIC, TCL, VISUAL TCL, JAVA, PERL, DBASE, ALGOL, BASIC, MACRO ASSEMBLER FORTH, DELPHI, etc.

As linguagens podem ser classificadas em NÍVEL BAIXO, MÉDIO e ALTO, essa classificação é feita de acordo com a capacidade da linguagem compreender instruções (códigos) escritos em "dialetos" próximos do inglês, e a linguagem que compreende somente linguagem de máquina (números binários) como o assembler, incapaz de ser compreendida pelo ser humano que não esteja treinado para tal.

O Delphi é uma linguagem visual, orientada a objetos (OOP) e derivada do Pascal, de Alto Nível, mais suave e de fácil manuseio pelo usuário (programador).

Sendo lançado a primeira versão do Delphi em 1995 foi considerada a melhor ferramenta no desenvolvimento para Windows.

Para se criar aplicativos começa-se com a montagem de componentes em janelas, como se fosse um programa gráfico, o usuário também pode utilizar componentes desenvolvidos por terceiros ou criar seus próprios componentes.

\subsection{UTILIZAÇÃo DA INTERFACE GRÁFICA}

Neste item é apresentada de forma bem sucinta a interface gráfica desenvolvida com a função de interpretar os dados geométricos discretizados em formato *.dxf (figura 55).

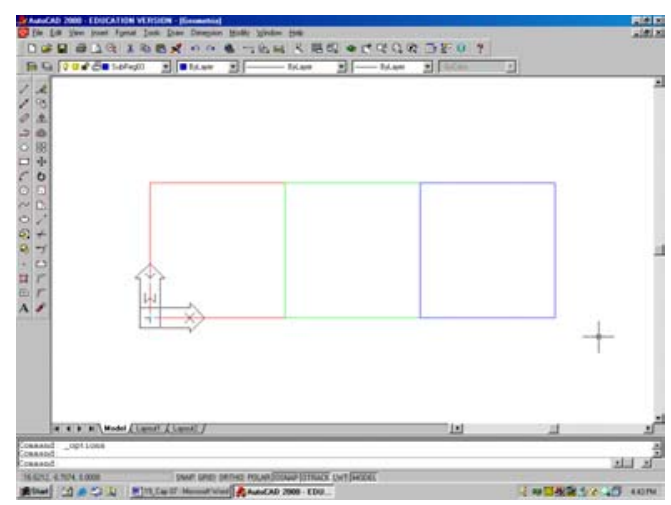

Figura 55 - Geometria do problema sendo discretizada em arquivo *.dxf. 
A interface inicial do programa que pode ser observada na figura 56 tem funções

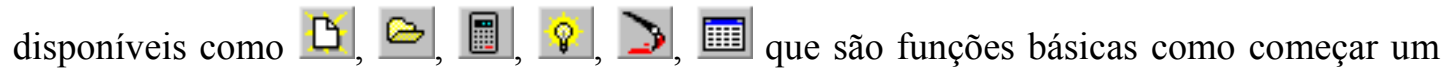
novo exemplo, abrir e alterar exemplos já pré existentes, calcular um modelo previamente gerado, interpretar os resultados do cálculo, visualizar os resultados do exemplo que se está simulando e por fim ver resultados de outros exemplos simulados anteriormente.

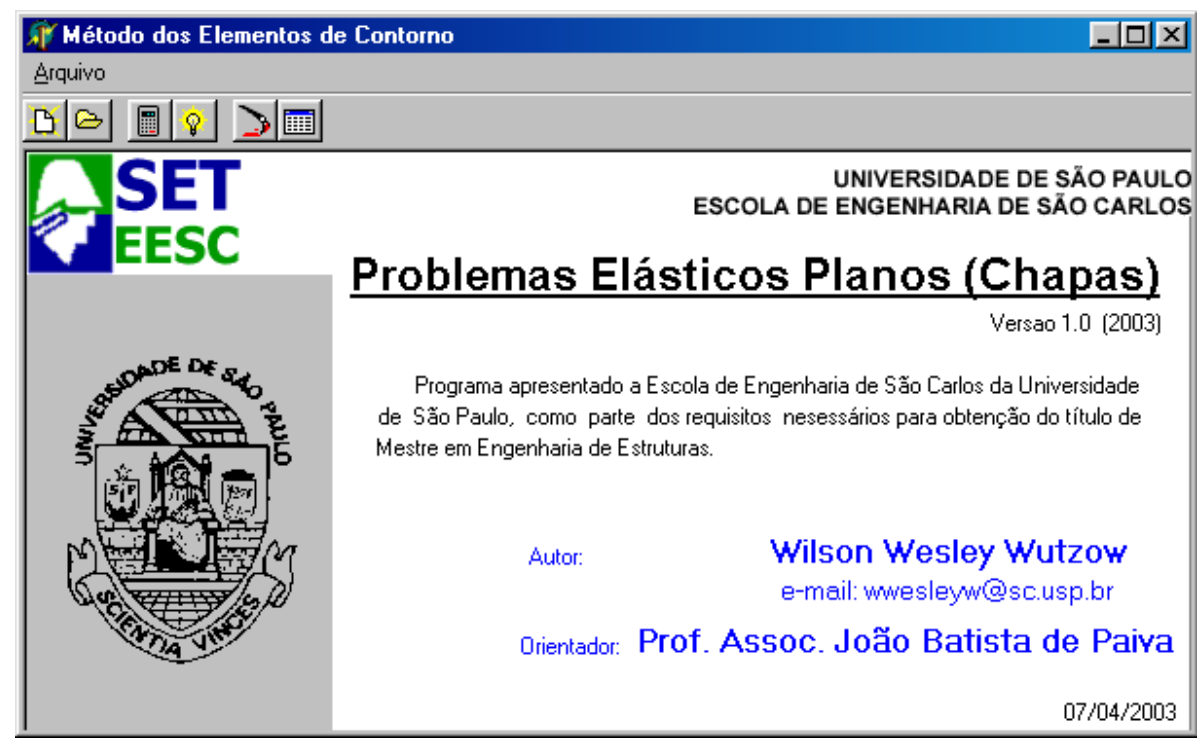

Figura 56 - Tela principal do programa.

Ao iniciar-se uma nova simulação, surge a figura 57 onde se escolhe o arquivo em formato *.dxf já previamente desenhado que contemple a geometria a ser simulada.

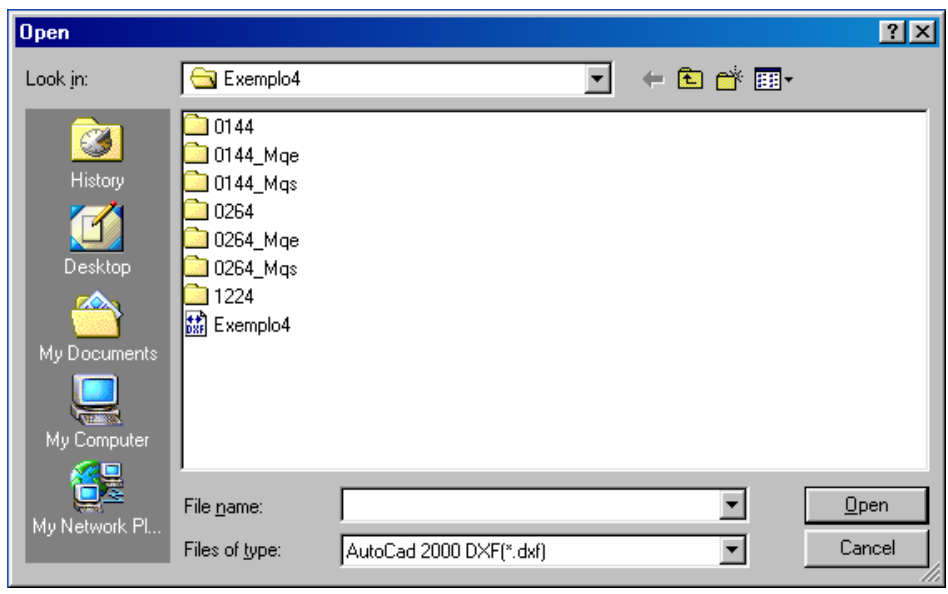

Figura 57 - Inserção dos dados geométricos do problema. 
Feita a leitura dos dados geométricos e escolhido o nome do arquivo a ser gerada para gravar o exemplo são feitas as configurações dos dados gerais do problema como: Número de sub-regiões, posicionamento dos pontos fontes, tipo de integração, aplicação ou não de suavização do contorno, etc. Estas escolhas são feitas na figura 58.

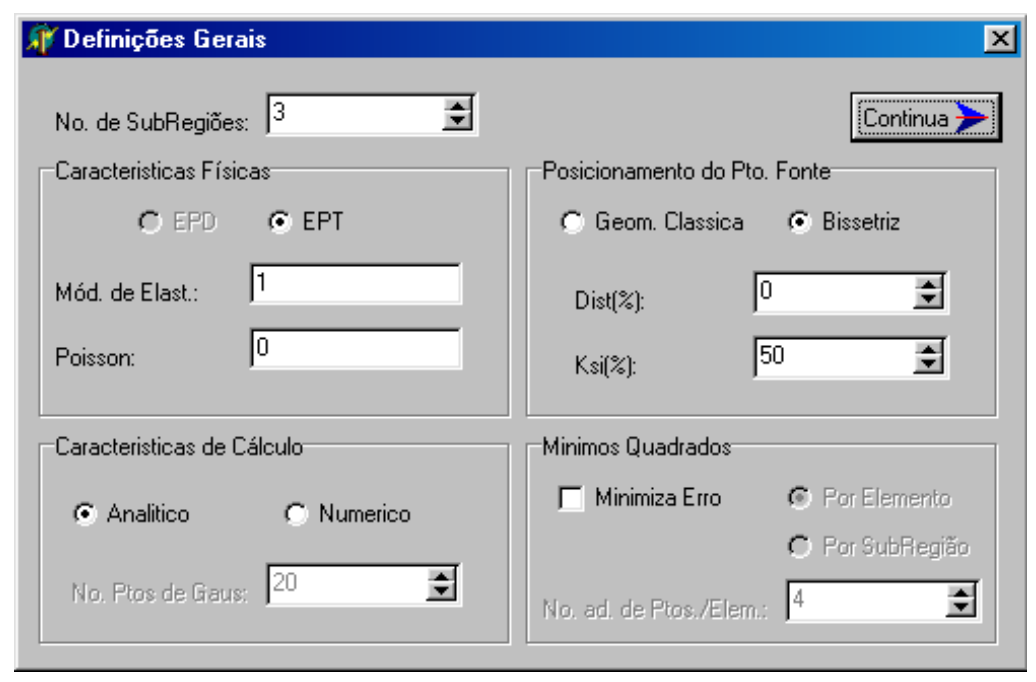

Figura 58 - Dados gerais do problema.

Feito isto são escolhidos as camadas (Layers) que serão empregados na discretização de cada uma das sub-regiões do problema. É também escolhido nesta fase as características físicas de cada uma das sub-regiões como: módulo de elasticidade e coeficiente de poison (Figura 59).

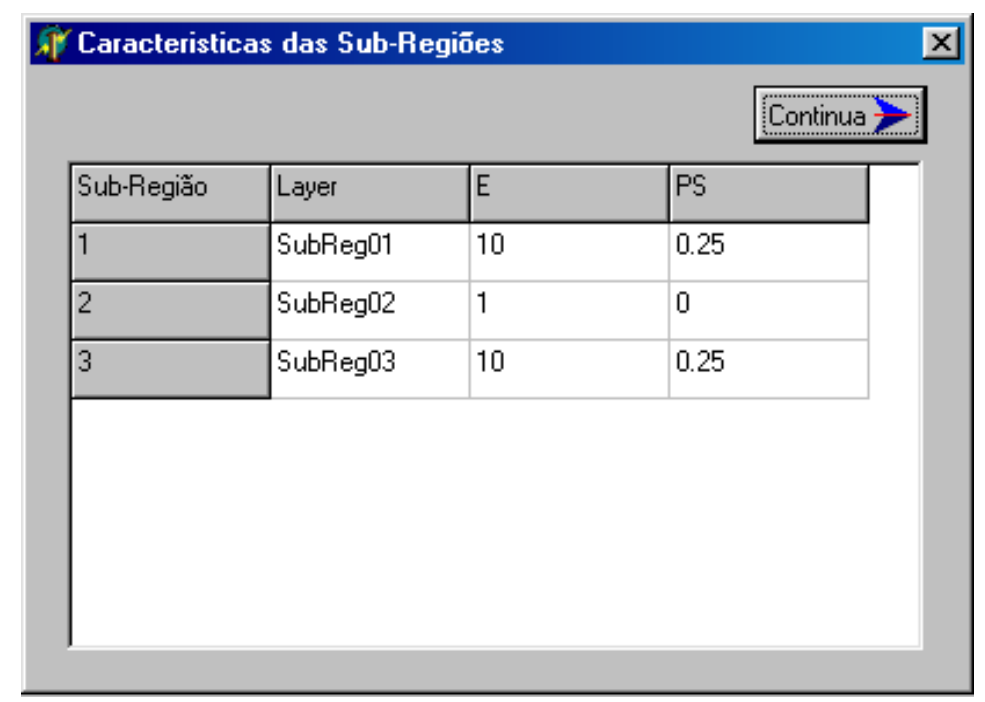

Figura 59 - configurações de cada uma das sub-regiões. 
Passa-se então a uma nova faze (figura 60) que tem como funções principais: gerar os elementos de contorno, geração de nós duplos, discretizar uma malha de domínio que até o momento é empregada apenas no pós-processamento para geração dos mapas e iso-linhas, etc.

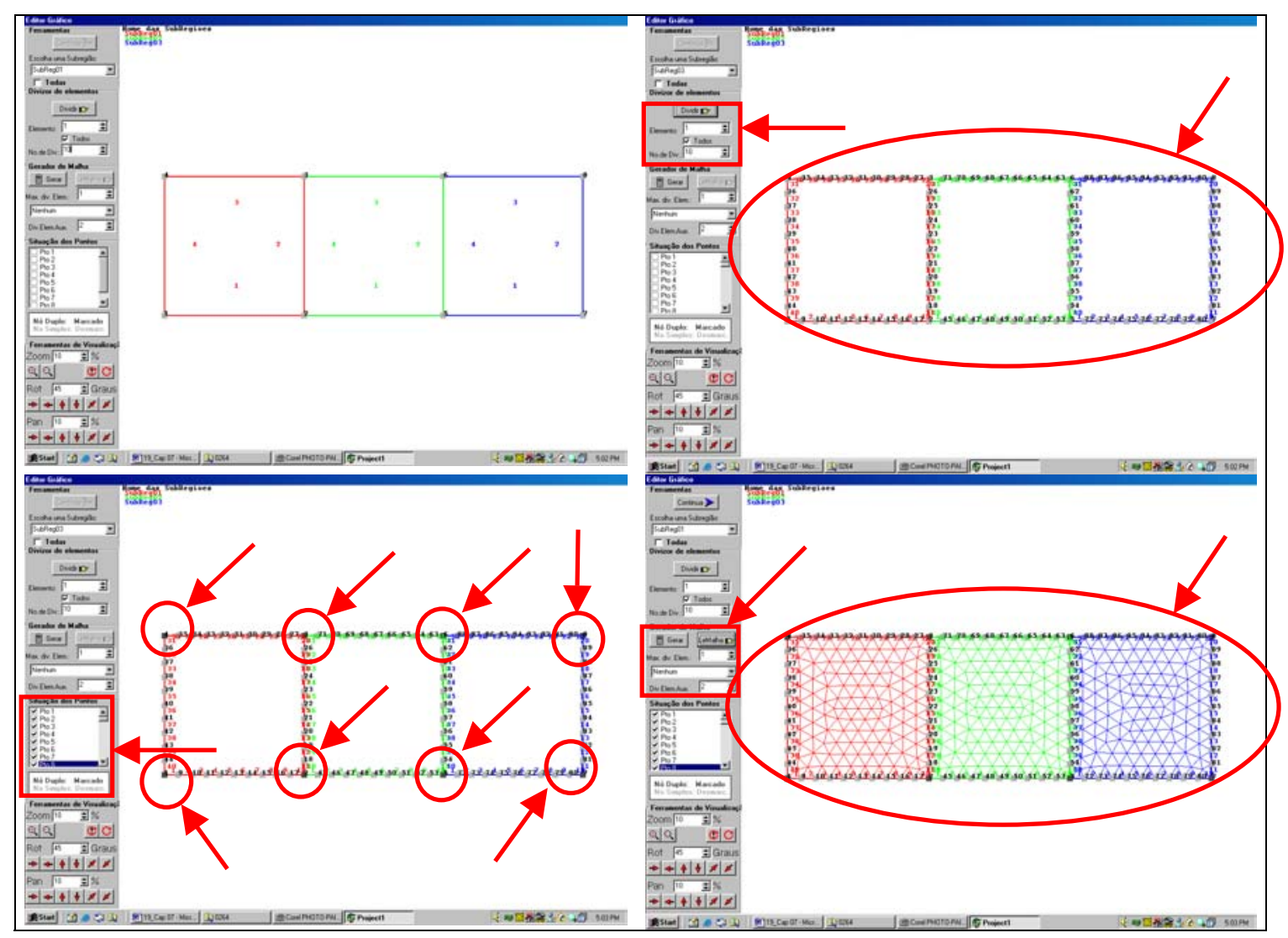

Figura 60 - Configurações relacionadas a discretização do problema.

Terminada esta etapa, é necessário agora informar as condições de contorno, seja carregamentos, vinculações ou deslocamentos. Para facilitar a configuração das condições de contorno, estes dados podem ser informados de forma coletiva desde que todos os nós em questão tenham a mesma condição de contorno (figura 61). 


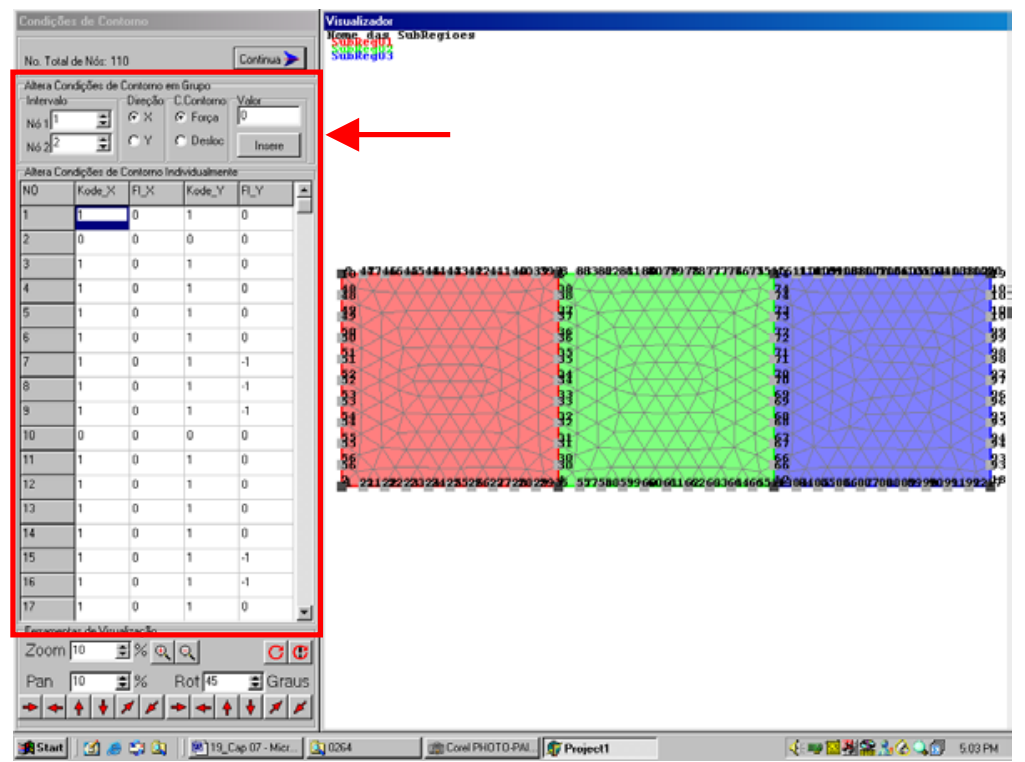

Figura 61 - Aplicação das condições de contorno.

Como o desenho é feito em uma plataforma desenvolvida em OpenGL (no Delphi) facilidade de visualização como aproximação(zoom), translação (pan) e rotação são possíveis de serem aplicadas de diversas maneiras, facilitando assim a visualização por exemplo da numeração do nó em que se deseja aplicar alguma condição de contorno (Figura $62)$.

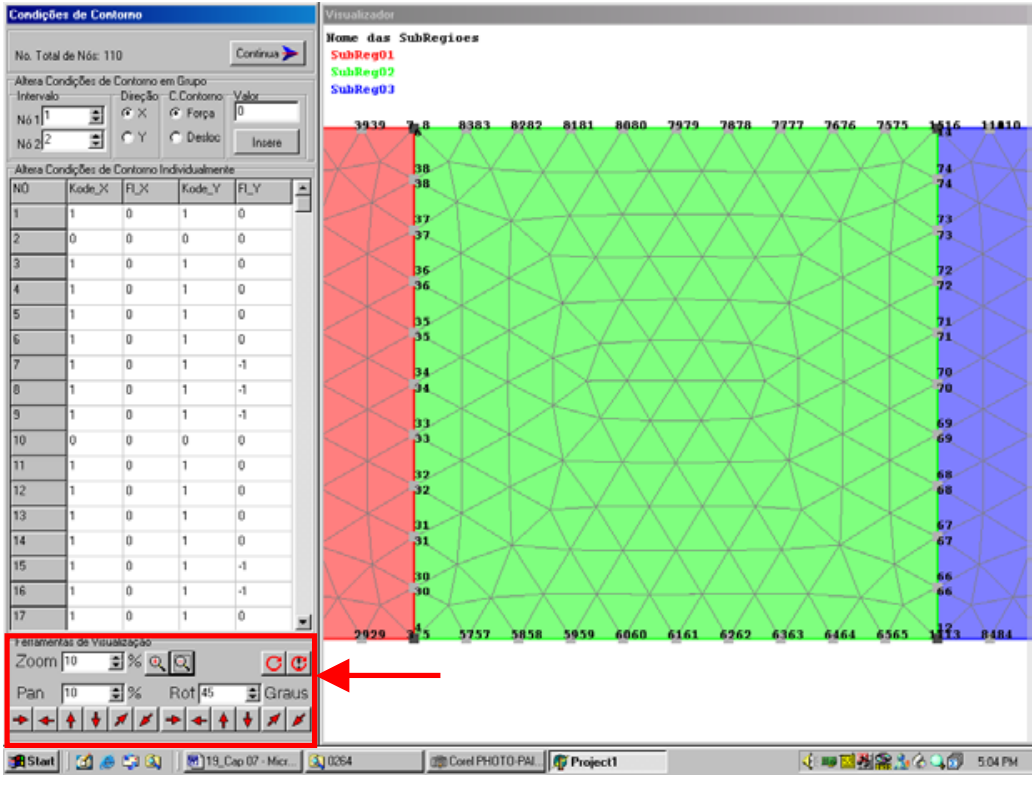

Figura 62 - Facilidade de vizualização. 
Ao terminar-se de definir as condições de contorno o programa retorna a janela principal onde é necessário chamar o botão 國 que informa os dados do problema ao programa desenvolvido em linhagem "Fortran" sendo este o núcleo de processamento, onde todos os cálculos são efetuados gerando os arquivos de resposta (Figura 63).

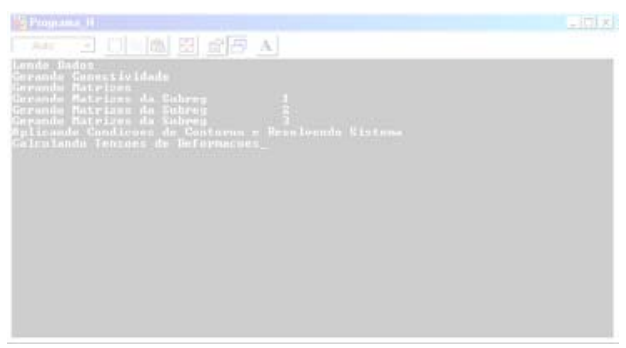

Figura 64 - Núcleo de processamento: onde os cálculos são efetuados.

Terminados os cálculos, deve-se então chamar o botão $\&$ que faz a leitura dos resultados de cálculo. Em seguida deve-se chamar o botão $\$$ possibilitando assim a visualização dos resultados (Figura 64).

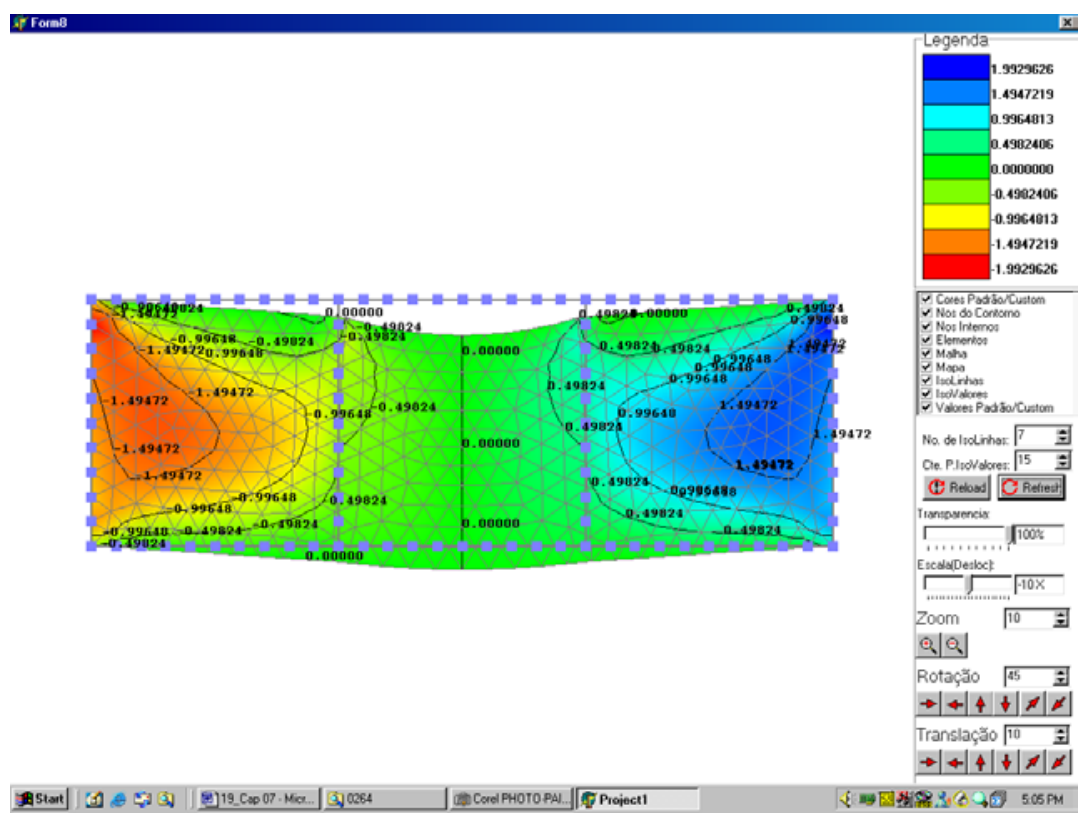

Figura 64 - Interface gráfica desenvolvida em Delphi com rotinas em OpenGl para visualização dos resultados.

Nesta interface várias facilidades de visualização foram desenvolvidas, possibilitando assim criar diversas escalas diferentes de cores, vários tipos de visualização de 
mapas coloridos, isolinhas, isovalores, graus de transparência, visualização da posição deslocada, aproximação, translação, rotação, configuração de escalas diferenciadas de valores. Tudo para facilitar na interpretação dos resultados. Os resultados que podem ser vistos são os mapas de tensão nos eixos globais ou os valores máximos e mínimos (autovalores), as deformações, também nos eixos globais ou valores extremos (autovalores), e os deslocamentos nos eixos globais. Algumas das funções descritas acima podem ser observadas na figura 65 .

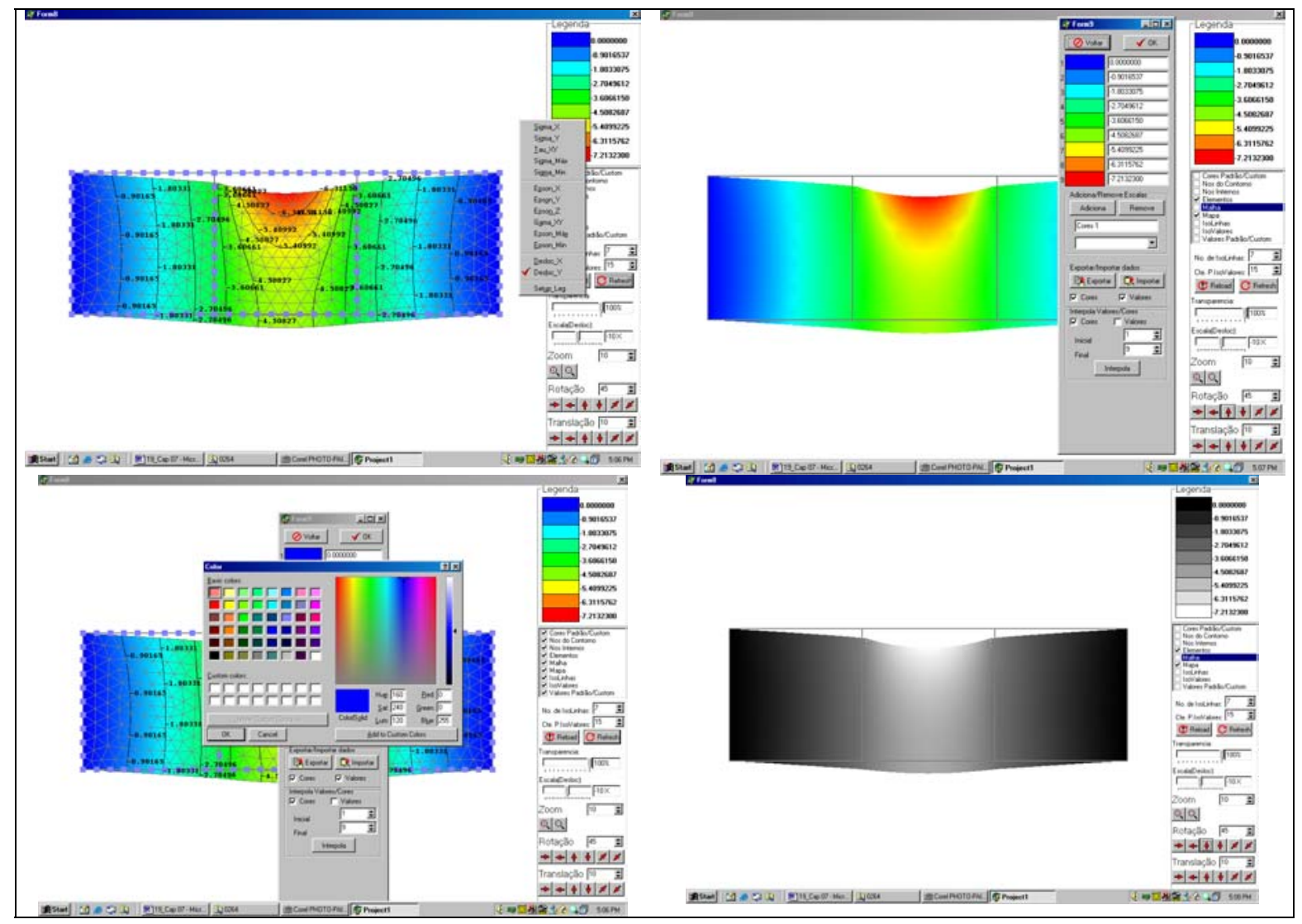

Figura 65 - Algumas configurações de visualização dos resultados.

\subsection{EXEMPLOS DE APLICAÇÃO}

Neste item são apresentados exemplos que demonstram os recursos de gerador de mapas e iso-linhas, os exemplos aqui apresentados são os mesmo já estudados nos capítulos anteriores. 


\section{Exemplo 1}

Os diagramas apresentados neste exemplo são gerados a partir do exemplo 1 do capítulo 5, na figura 66 o problema é apresentado novamente.

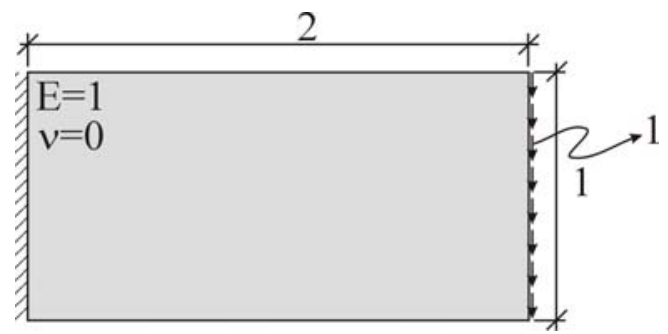

Figura 66 - Dados do exemplo 1 do capítulo 5.

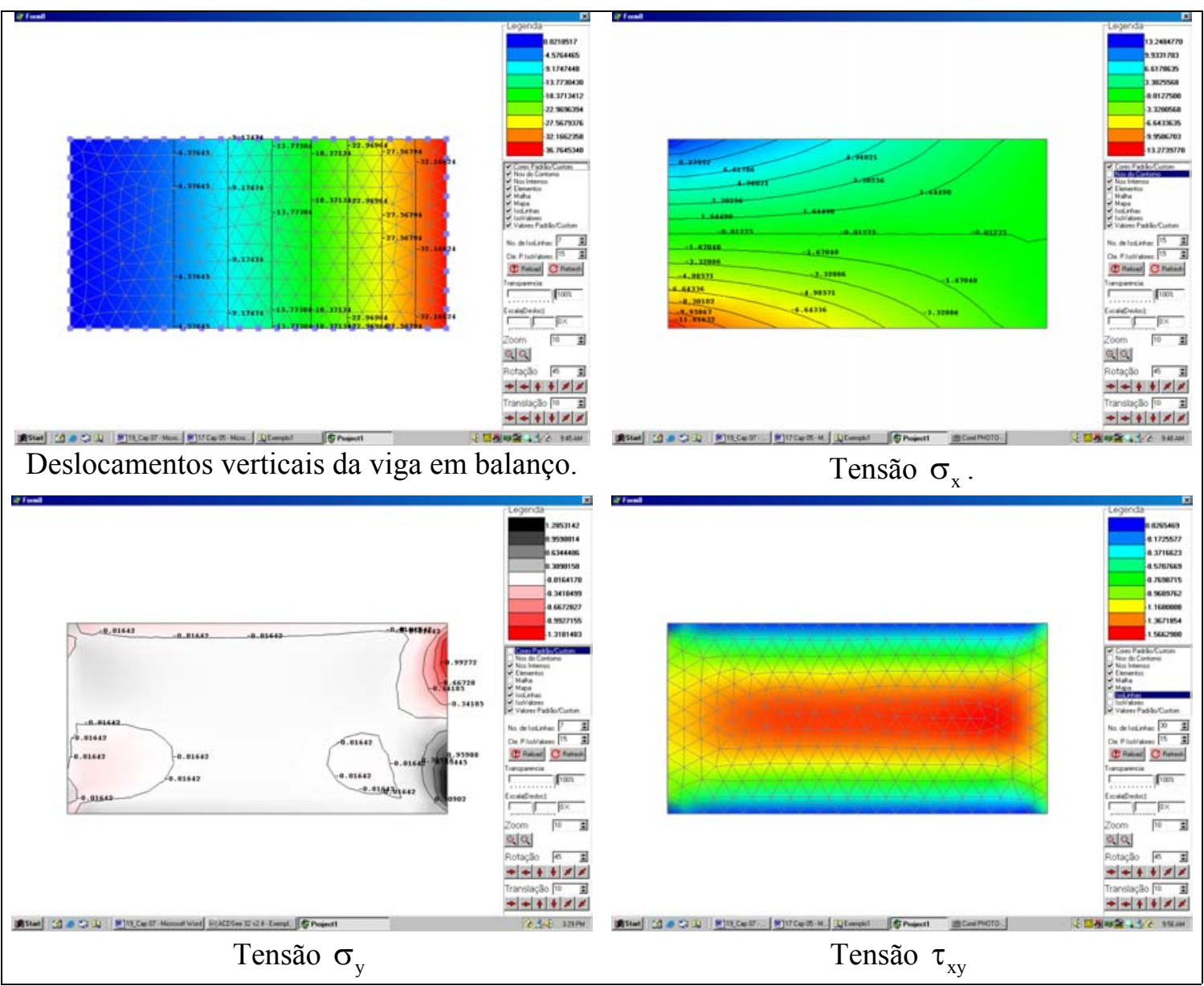

Figura 67 - Representação gráfica dos resultados do exemplo 1 do capítulo 5.

Como se pode observar na figura 67 várias configurações de visualização podem ser escolhidas, com ou sem malha iso-linhas, iso-valores, ou personalizando a paleta de cores. 


\section{Exemplo 2}

Abaixo, na figura 68 é apresentado novamente o exemplo 2 que está contido no capítulo 5, e à seguir são apresentados os resultados de forma gráfica na figura 69 .

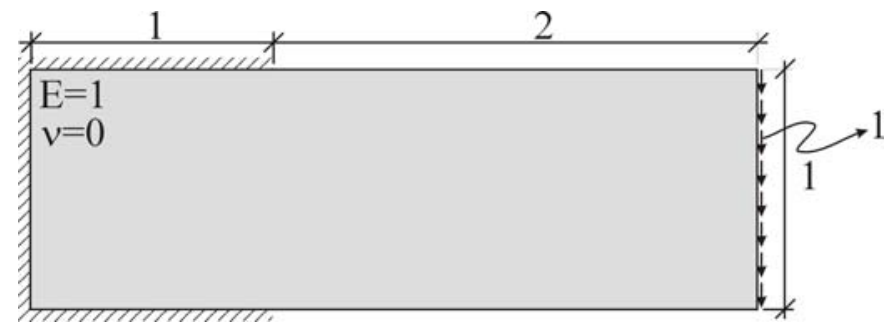

Figura 68 - Dados do exemplo 2 do capítulo 5.

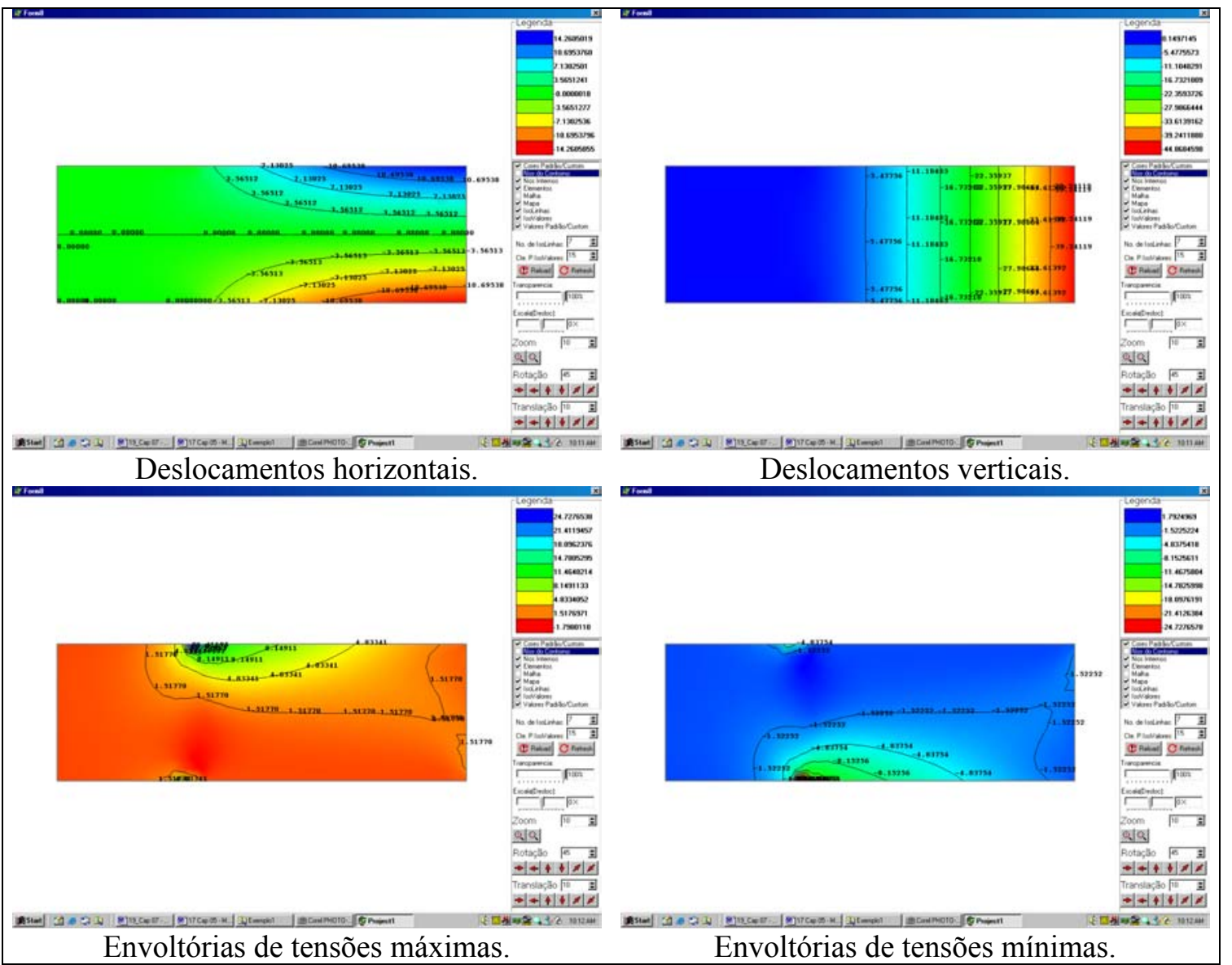

Figura 69 - Representação gráfica dos resultados do exemplo 2 do capítulo 5.

Além dos digramas de tensão, deformação e deslocamentos, outros diagramas como envoltórias de tensões máximas e mínimas podem ser observados (figura 70). 


\section{Exemplo 3}

Através dos resultados do exemplo 3 do capítulo 5 (figura 70) apresentados de forma gráfica na figura 71, é apresentado o recurso de transparência do mapa de cores dando assim maior destaque às iso-linhas e iso-valores que são impresos.

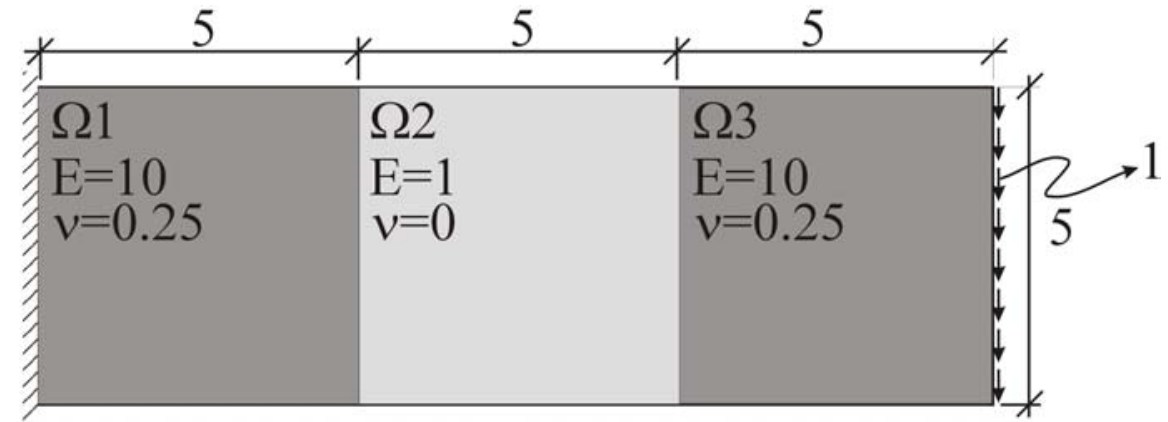

Figura 70 - Dados do exemplo 3 do capítulo 5.

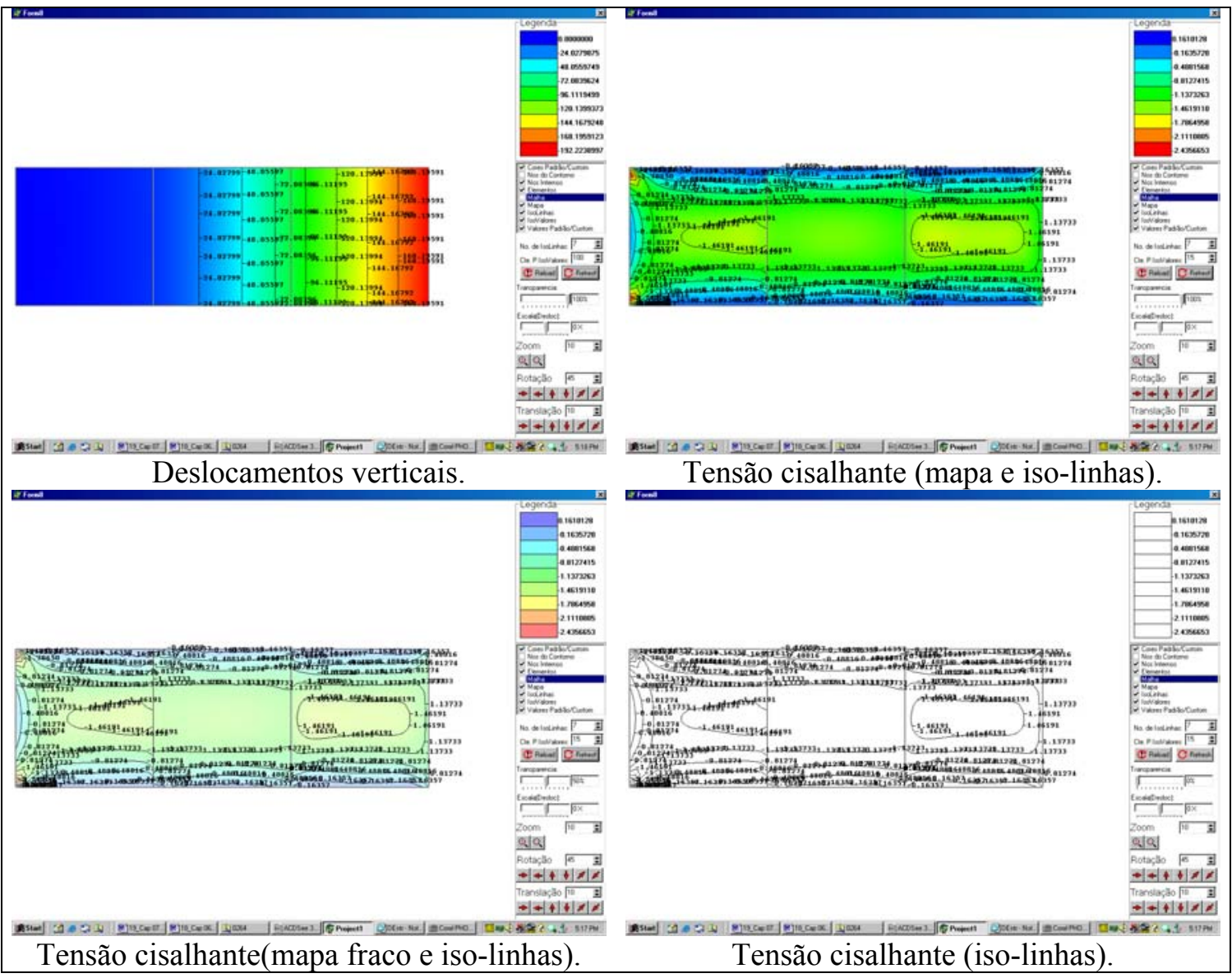

Figura 71 - Recursos de transparência dos mapas. 


\section{Exemplo 4}

Na figura 73 estão impressos os resultados do exemplo 1 apresentado no capítulo 6 (figura 72). Na figura 73 estão os diagramas de $\sigma_{x}, \sigma_{y}, \tau_{x y}$ e $U_{y}$.

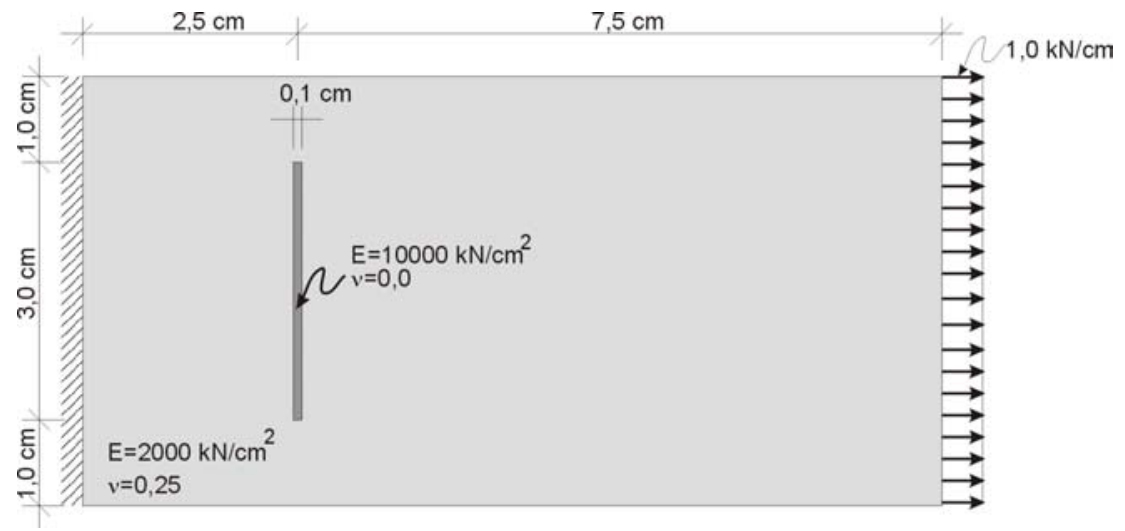

Figura 72 - Dados do problema 1 do capítulo 6.

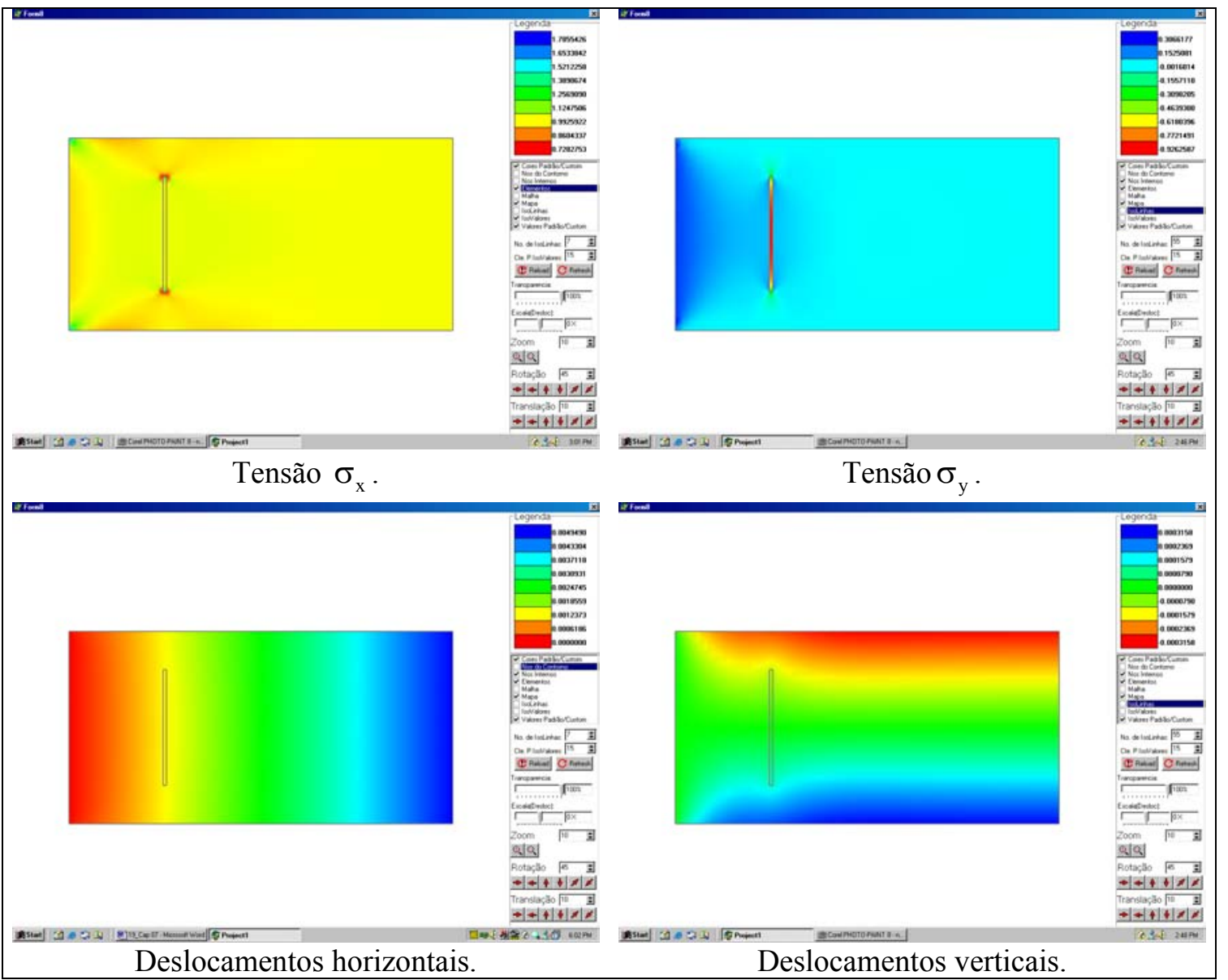

Figura 73 - Representação gráfica dos resultados do exemplo 1 do capítulo 6. 
$\mathrm{Na}$ figura 74 são apresentados alguns recursos que visualizam melhor concentração de tensões, para os dois casos a discretização do contorno é a mesma, somente a malha de pós-processamento é que foi alterada, no primeiro casos menos discretizada com gerador de malha, no segundo caso mais discretizada gerada no arquivo dxf de entrada de dados. Além da discretização da malha de pós processamento, outro recurso empregado para melhorar a vizualização foi alterar a configuração da escala de cores. Os resultados são apresentados também em escala monocromática.
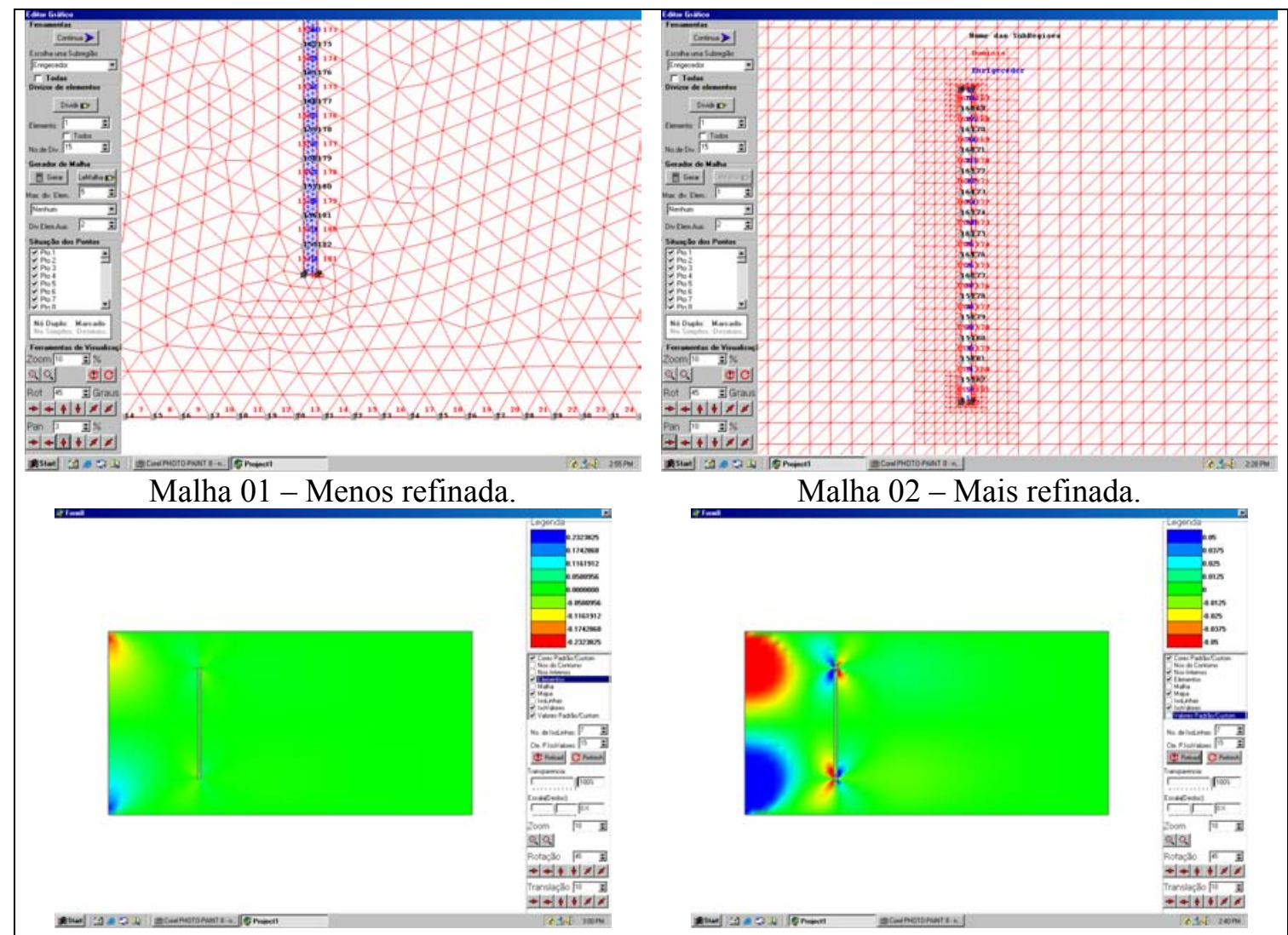

Tensão cisalhante (malha 01)

Tensão cisalhante (malha 02).

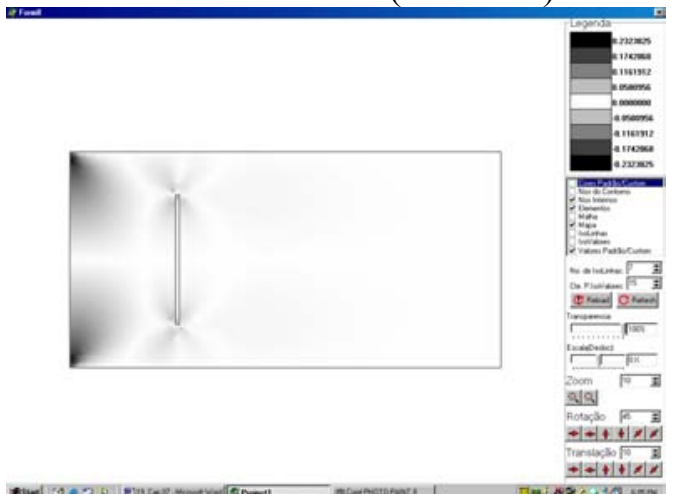

Tensão cisalhante (malha 01).

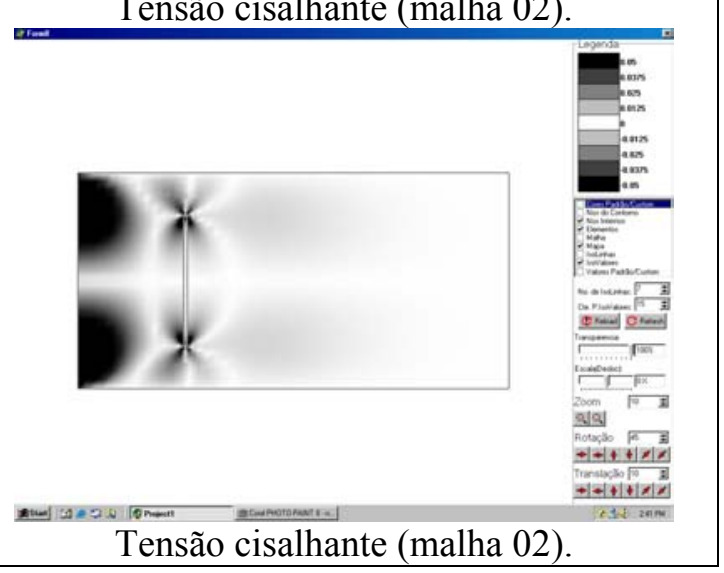

Figura 74 - Recursos que melhoram a visualização de concentração de tensões. 


\section{Exemplo 5}

A seguir, na figura 76, são apresentados os digramas de deslocamento verticais (já na posição deformada) e tensões $\sigma_{\mathrm{x}}, \sigma_{\mathrm{y}}$ e $\tau_{\mathrm{xy}}$ que representam os resultados obtidos para o exemplo 2 do capítulo 6 (Figura 75).

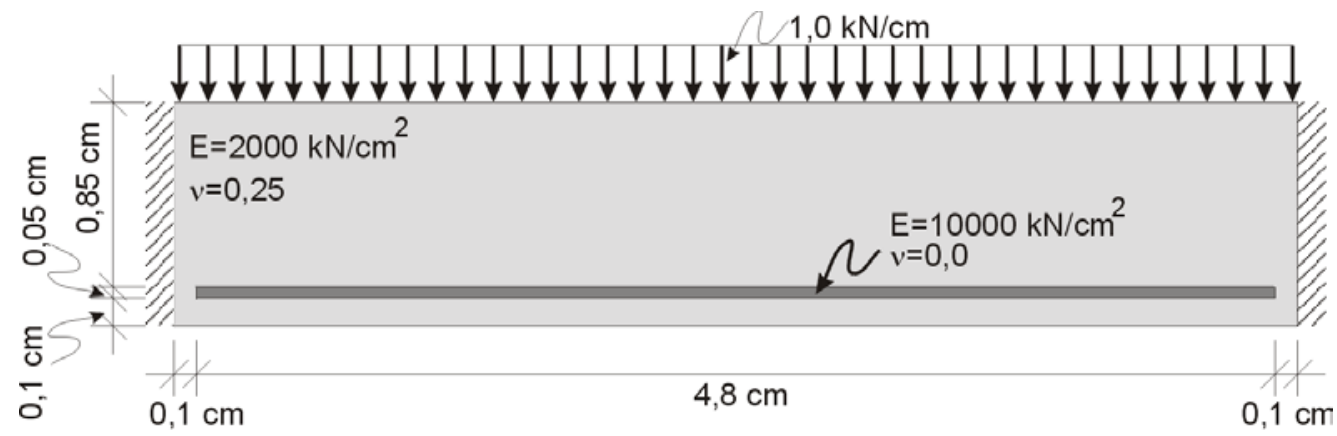

Figura 75 - Dados do problema 2 do capítulo 6.

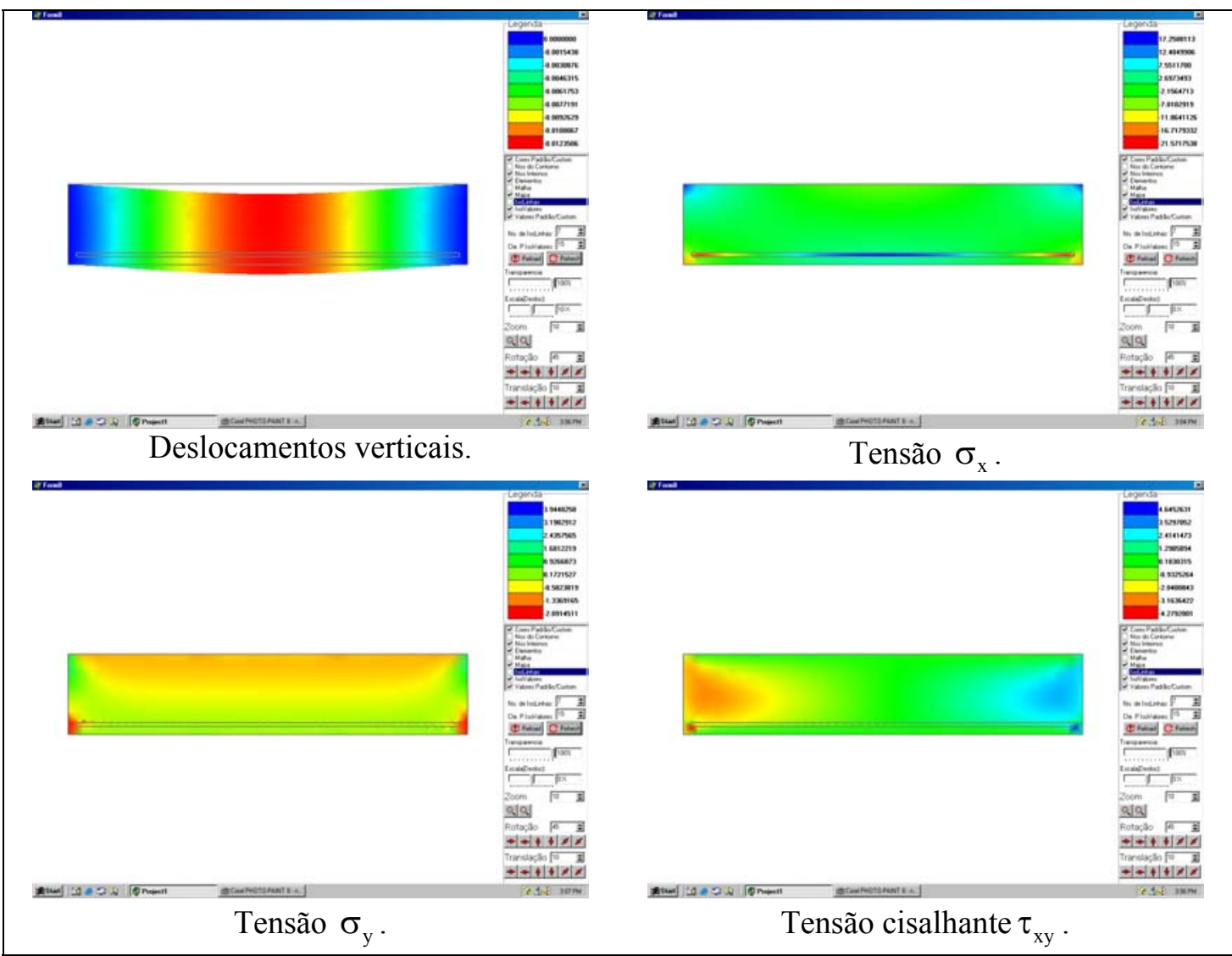

Figura 76 - Representação gráfica dos resultados do exemplo 2 do capítulo 6. 


\section{Exemplo 6}

Nas figuras 77 são apresentados os deslocamentos, as tensões e as deformações encontradas no exemplo 3 do capítulo 6 (Figura 78):

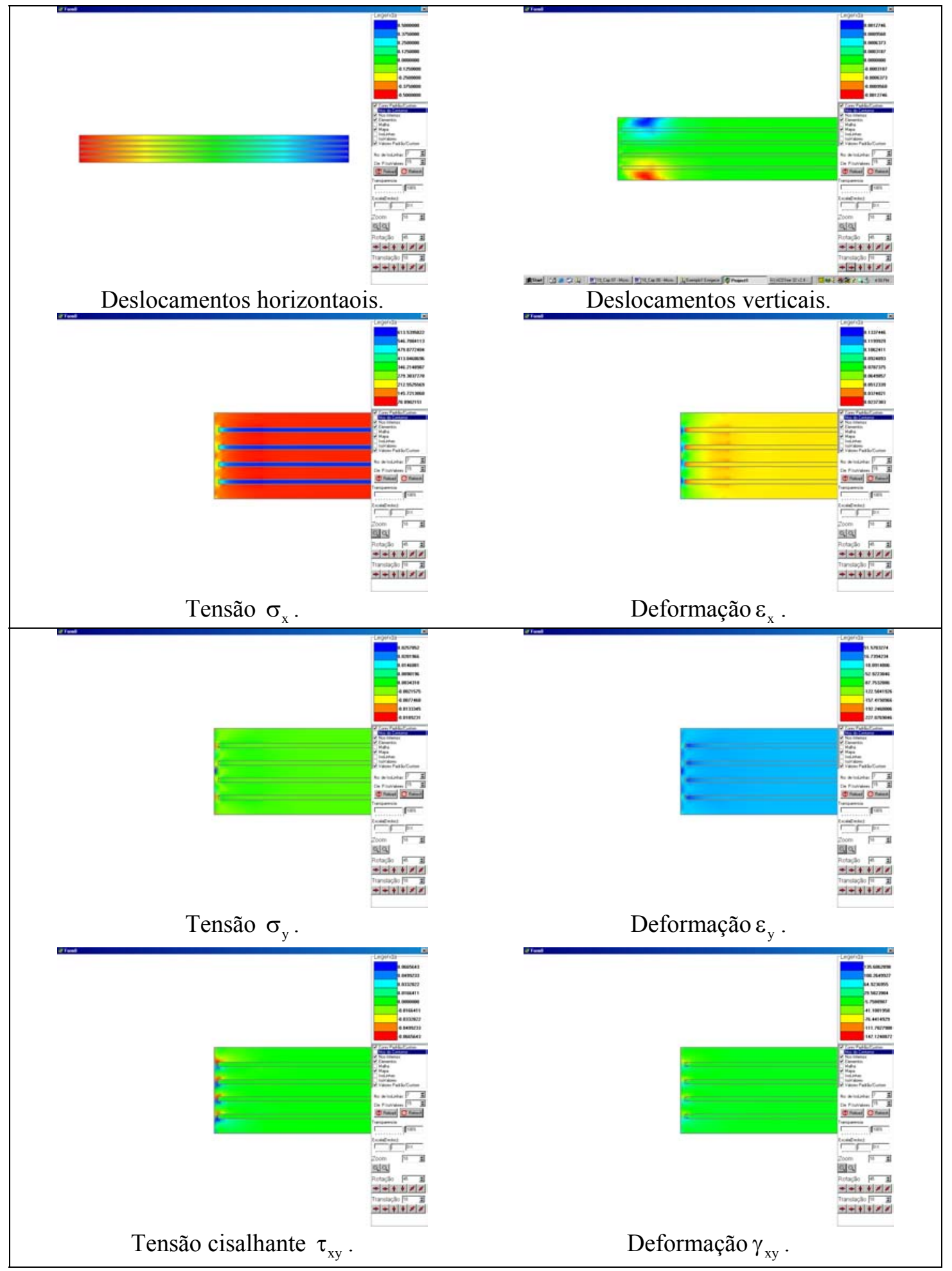

Figura 77 - Representação gráfica dos resultados do exemplo 3 do capítulo 6. 


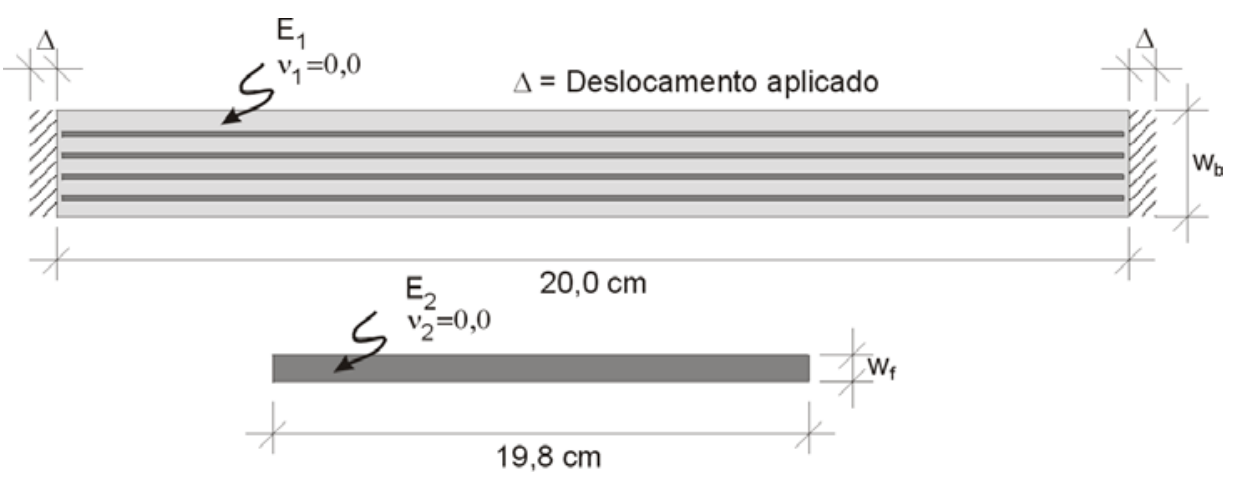

Figura 78 - Dados do problema 3 do capítulo 6.

\section{4 - Conclusões Parciais}

Apesar da grande quantidade de recursos implementados com o objetivo de facilitar a utilização do programa, várias facilidades foram deixadas de abordar, isto porque, apesar do assunto ser interessante, não desejávamos tirar o foco da dissertação do modelo estudado (enrijecedores BEM/BEM com integração analíticas).

Contudo o programa desenvolvido demonstrou-se versátil facilitando a geração de modelos e simplificando a visualização dos resultados. Devido ao tempo escasso, não se preocupou em otimizar determinadas rotinas que tornariam o programa ainda mais ágil principalmente para exemplos de grande discretização.

O gerador de malha aqui empregada para pós-processamento, com as devidas alterações, poderia facilmente ser empregado para aplicação também de cargas de domínio. A facilidade de poder escolher entre o gerador automático de malhas ou gerar manualmente a malha em arquivo dxf em determinadas circunstâncias facilitam a discretização de malha em regiões críticas.

Uma outra forma que poderia levar a bons resultados na hora de gerar as iso-linhas seria empregar curvas do tipo spline juntamente com o emprego da equação dos gradientes de tensão. 
Este estudo demonstra que a análise de estruturas planas (chapas) enrijecidas pode ser feita através de uma formulação envolvendo apenas elementos de contorno, dispensando assim a combinação com o MEF tradicionalmente empregada. O problema pode ser discretizado por várias sub-regiões que representam as chapas e os enrijecedores acoplados entre si. Esta solução não é inédita, já tem sido aplicada por diversos pesquisados, contudo neste trabalha através da integração analítica buscou-se obter resultados que representassem melhor o problema a ser simulado, evitando assim possíveis perturbações decorrentes da esbelteza de enrijecedores finos. Pode-se ainda citar que tais variáveis (agora condensadas) poderiam ainda ser expressas de outra forma possibilitando ainda a redução de graus de liberdade do sistema de equações.

Entretanto para espessuras muito pequenas de enrijecedores, com o objetivo de evitar perturbações espúrias, empregou-se ainda a condensação das variáveis do contorno, transformando as mesmas em incógnitas resultantes (para as forças) e valores da linha média (para os deslocamentos). Como se pode observar esta técnica, quando aplicada, melhorou os resultados.

Sobre a técnica de suavização descrita neste estudo, obteve-se bons resultados conseguindo alcançar resultados mais precisos com discretizações mais pobres, pode-se facilmente observar também, que das duas formas em que a técnica foi aqui empregada, a técnica aplicada por elemento levou a resultados (na maioria dos casos) melhores do que quando aplicada por sub-região. Sobre o alívio de possíveis perturbações espúrias nos resultados, motivo pelo qual a técnica se chamar "suavização do contorno", bons resultados foram observados no estudo desenvolvido por BOTTA \& VENTURINI (2003), onde era empregado acoplamento MEC/MEF, situação essa indicada para aplicação da técnica. 
Sobre as facilidades gráficas aqui empregadas pode-se mencionar que recursos como leitura de dados via arquivo de formato dxf, geração automática de malhas, e interpretadores geométricos do contorno aqui desenvolvidos, juntamente com as facilidades proporcionadas pela criação de uma interface gráfica desenvolvida em OpenGL, forneceram ao programa a agilidade e as facilidades desejadas inicialmente.

Apesar da grande variedade funções e rotinas que ainda poderiam ser desenvolvidas para representação gráfica dos resultados, os recursos desenvolvidos foram mais do que suficientes para a boa visualização dos resultados. É bem verdade que os resultados analisados pelos mapas coloridos ou pelas iso-linhas nos levam a apenas uma análise qualitativa do mesmo. Mas tal análise é necessária a fim de proporcionar segurança e confiabilidade aos resultados numéricos que posteriormente podem ser analisados do forma quantitativa. 


\section{TEOREMA DE BETTI}

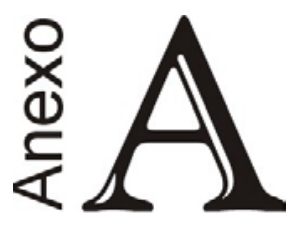

Considere um corpo em equilíbrio quando submetido à ação de dois estados de carregamento, cada um deles levando a um dos estados de tensão definidos a seguir:

- Para um estado de carregamento (1), tensões $\sigma_{\mathrm{ij}}^{(1)}$ que dão origem ao conjunto de deformações $\varepsilon_{\mathrm{ij}}^{(1)}$.

- Para um estado de carregamento (2), tensões $\sigma_{\mathrm{ij}}^{(2)}$ que dão origem ao conjunto de deformações $\varepsilon_{\mathrm{ij}}^{(2)}$.

A reciprocidade de Betti garante que o trabalho feito pelas tesões do primeiro sistema sobre as deformações do segundo sistema é igual ao trabalho efetuado pelas tensões do segundo sistema sobre as deformações do primeiro sistema, ou seja,

$$
\int_{\Omega} \sigma_{\mathrm{ij}}^{1} \varepsilon_{\mathrm{ij}}^{2} \mathrm{~d} \Omega=\int_{\Omega} \sigma_{\mathrm{ij}}^{2} \varepsilon_{\mathrm{ij}}^{1} \mathrm{~d} \Omega
$$

Isto é facilmente demonstrado a seguir.

Partindo-se da lei de Hooke, tem-se:

$$
\sigma_{\mathrm{ij}}=\mathrm{C}_{\mathrm{ijk} \mathrm{k}} \varepsilon_{\mathrm{kl}}
$$


que particularizada para um material isotrópico no estado plano de deformação é:

$\sigma_{\mathrm{ij}}=\frac{2 \mathrm{G} v}{1-2 v} \varepsilon_{\mathrm{kk}} \delta_{\mathrm{ij}}+2 \mathrm{G} \varepsilon_{\mathrm{ij}}$

Logo

$\sigma_{\mathrm{ij}}{ }^{1} \varepsilon_{\mathrm{ij}}{ }^{2}=\left(\frac{2 \mathrm{G} v}{1-2 \nu} \varepsilon_{\mathrm{kk}}{ }^{1} \delta_{\mathrm{ij}}+2 \mathrm{G} \varepsilon_{\mathrm{ij}}{ }^{1}\right) \varepsilon_{\mathrm{ij}}{ }^{2}$

$\sigma_{\mathrm{ij}}{ }^{1} \varepsilon_{\mathrm{ij}}{ }^{2}=\frac{2 \mathrm{G} v}{1-2 v} \varepsilon_{\mathrm{kk}}{ }^{1} \delta_{\mathrm{ij}} \varepsilon_{\mathrm{ij}}{ }^{2}+2 \mathrm{G} \varepsilon_{\mathrm{ij}}{ }^{1} \varepsilon_{\mathrm{ij}}{ }^{2}$

como

$\left\{\begin{array}{l}\delta_{\mathrm{ij}} \varepsilon_{\mathrm{ij}}{ }^{2}=\varepsilon_{\mathrm{ii}}{ }^{2}=\varepsilon_{\mathrm{kk}}{ }^{2} \\ \varepsilon_{\mathrm{kk}}{ }^{1}=\varepsilon_{\mathrm{ii}}{ }^{1}=\delta_{\mathrm{ij}} \varepsilon_{\mathrm{ij}}{ }^{1}\end{array}\right.$

tem-se

$$
\begin{aligned}
& \sigma_{\mathrm{ij}}{ }^{1} \varepsilon_{\mathrm{ij}}{ }^{2}=\frac{2 \mathrm{G} v}{1-2 v} \delta_{\mathrm{ij}} \varepsilon_{\mathrm{ij}}{ }^{1} \varepsilon_{\mathrm{kk}}{ }^{2}+2 \mathrm{G} \varepsilon_{\mathrm{ij}}{ }^{1} \varepsilon_{\mathrm{ij}}{ }^{2} \\
& \sigma_{\mathrm{ij}}{ }^{1} \varepsilon_{\mathrm{ij}}{ }^{2}=\left(\frac{2 \mathrm{G} v}{1-2 v} \delta_{\mathrm{ij}} \varepsilon_{\mathrm{kk}}{ }^{2}+2 \mathrm{G} \varepsilon_{\mathrm{ij}}{ }^{2}\right) \varepsilon_{\mathrm{ij}}{ }^{1} \\
& \sigma_{\mathrm{ij}}{ }^{1} \varepsilon_{\mathrm{ij}}{ }^{2}=\sigma_{\mathrm{ij}}{ }^{2} \varepsilon_{\mathrm{ij}}{ }^{1}
\end{aligned}
$$




\section{FunÇão Delta de DiraC}

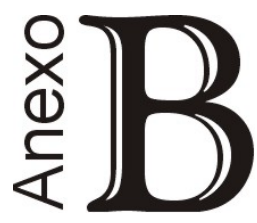

A função Delta de Dirac tem suas propriedades estudadas na Teoria de Funções Generalizadas e constitui uma ferramenta capaz de representar forças concentradas na Teoria da Elasticidade ou fontes concentradas na Teoria de Potencial. Ela pode ser facilmente deduzida a partir da diferenciação da função "Have Size" ou função degrau.

A função Delta de Dirac está representada na figura abaixo :

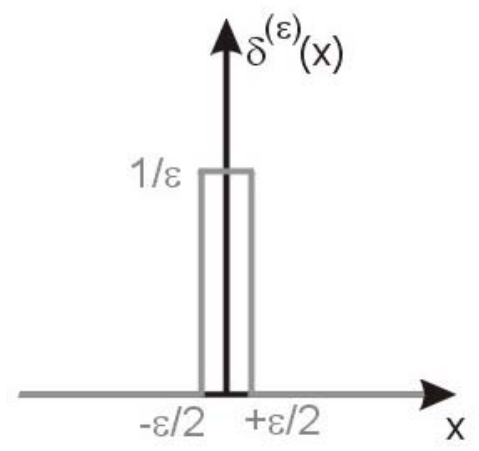

Figura B.1:

Sendo a função $\delta^{(\varepsilon)}(\mathrm{x})$, podendo ser definida da seguinte maneira :

$$
\delta^{(\varepsilon)}(\mathrm{x})=\left\{\begin{array}{c}
1 / \varepsilon, \text { para }-\varepsilon / 2<\mathrm{x}<\varepsilon / 2 \\
0, \text { para } \quad|\mathrm{x}|>\varepsilon / 2
\end{array}\right.
$$

onde $\varepsilon$ é um número positivo. 
Tem-se a integral:

$$
I=\int_{-\infty}^{+\infty} \delta^{(\varepsilon)}(x) f(x) d x
$$

onde $\mathrm{f}(\mathrm{x})$ é uma função qualquer bem definida em $\mathrm{x}=0$. Se $\varepsilon$ for suficientemente pequeno, a variação de $\mathrm{f}(\mathrm{x})$ no intervalo efetivo de integração $[-\varepsilon / 2, \varepsilon / 2]$ é negligenciável e $f(x)$ permanece praticamente igual a $f(0)$, de forma que:

$$
I \cong f(0) \int_{-\infty}^{+\infty} \delta(x) d x=f(0)
$$

A aproximação é tanto melhor quanto menor for $\varepsilon$. Na passagem ao limite, quando $\varepsilon \rightarrow 0$, obtém-se a definição da função Delta de Dirac pela relação

$$
\int_{-\infty}^{+\infty} \delta(x) f(x) d x=f(0)
$$

válida para qualquer função $\mathrm{f}(\mathrm{x})$ definida na origem, uma definição mais geral seria

$$
\int_{-\infty}^{+\infty} \delta\left(x-x_{0}\right) d x=f\left(x_{0}\right)
$$

O conceito da função Delta de Dirac pode ser facilmente estendido a domínios ndimensionais. Considerando-se uma função f que depende da localização de cada ponto no corpo, define-se $\delta(\mathrm{p}, \mathrm{Q})$, como a função Delta de Dirac, quando são válidas as seguintes propriedades:

$$
\begin{aligned}
& \delta(\mathrm{p}, \mathrm{Q})=\left\{\begin{array}{l}
\infty, \mathrm{sep}=\mathrm{Q} \\
0, \text { se } \mathrm{p} \neq \mathrm{Q}
\end{array}\right. \\
& \int_{\Omega} \mathrm{g}(\mathrm{Q}) \delta(\mathrm{p}, \mathrm{Q}) \mathrm{d} \Omega=\mathrm{g}(\mathrm{p})
\end{aligned}
$$

A função Delta de Dirac também pode ser representada da seguinte maneira:

$$
\delta(\mathrm{p}, \mathrm{Q})=\Delta^{\mathrm{p}} .
$$




\section{FunÇÃo Delta de KRONECKER}

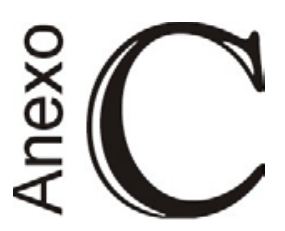

$\mathrm{Na}$ formulação de certos problemas, é muitas vezes necessário empregar-se o conceito de matriz identidade através de uma função que a represente. Este papel é muito bem desempenhado pela função Delta de Kronecker que é definida por:

$$
\delta_{\mathrm{ij}}=\delta_{\mathrm{ji}}=\left\{\begin{array}{l}
1, \text { se } \mathrm{i}=\mathrm{j} \\
0, \text { se } \mathrm{i} \neq \mathrm{j}
\end{array}\right.
$$

Ou seja, a matriz

$$
\left[\delta_{\mathrm{ij}}\right]=\left[\begin{array}{cccc}
\delta_{11} & \delta_{12} & \cdots & \delta_{1 \mathrm{~m}} \\
\delta_{21} & \delta_{22} & \cdots & \delta_{2 \mathrm{~m}} \\
\vdots & \vdots & \ddots & \vdots \\
\delta_{\mathrm{n} 1} & \delta_{\mathrm{n} 2} & \cdots & \delta_{\mathrm{nm}}
\end{array}\right]
$$

equivale à matriz identidade.

É imediato verificar que qualquer que seja a matriz quadrada $M_{i j}$ e o vetor $A_{i}$ se tem

$$
\begin{aligned}
& \delta_{\mathrm{ij}} \mathrm{A}_{\mathrm{j}}=\mathrm{A}_{\mathrm{i}} \\
& \delta_{\mathrm{ij}} \mathrm{M}_{\mathrm{jk}}=\mathrm{M}_{\mathrm{ik}}=\mathrm{M}_{\mathrm{ij}} \delta_{\mathrm{jk}}
\end{aligned}
$$




$$
\begin{aligned}
& \delta_{\mathrm{ik}} \delta_{\mathrm{kj}}=\delta_{\mathrm{ij}} \\
& \delta_{\mathrm{ij}}=\delta_{\mathrm{ji}}
\end{aligned}
$$

sendo a última expressão indicativa de que o símbolo de Kronecker é uma multiplicidade simétrica. 


\section{INTEGRAiS ANALÍtiCAS NÃo SingUlaReS}

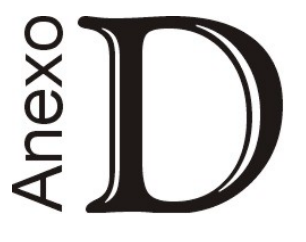

Para auxiliar na dedução, algumas constantes foram agrupadas de forma ordenada como é descrito abaixo:

$$
\begin{aligned}
& \mathrm{K}_{1}=\frac{-1}{8 \pi \mathrm{G}(1-v)} \\
& \mathrm{K}_{2}=(3-4 v) \\
& \mathrm{K}_{3}=\frac{-1}{4 \pi(1-v)} \\
& \mathrm{K}_{4}=(1-2 v) \\
& \mathrm{K}_{5}=(1-4 v) \\
& \mathrm{K}_{6}=(3-4 v) \\
& \mathrm{K}_{7}=\frac{\mathrm{G}}{2 \pi(1-v)} \\
& \phi_{1}=\left(1-\frac{\mathrm{y}_{\mathrm{s}}}{\mathrm{L}}\right) \\
& \mathrm{L}
\end{aligned}
$$


obs.: $\phi_{1}$ e $\phi_{2}$ não são as funções de forma do problema mas si as partes constantes das mesmas que foram usadas para simplificar a apresentação dos resultados.

\section{D.1 Ponto FONTE NÃo ALINHAdo COM O ELEMENTO}

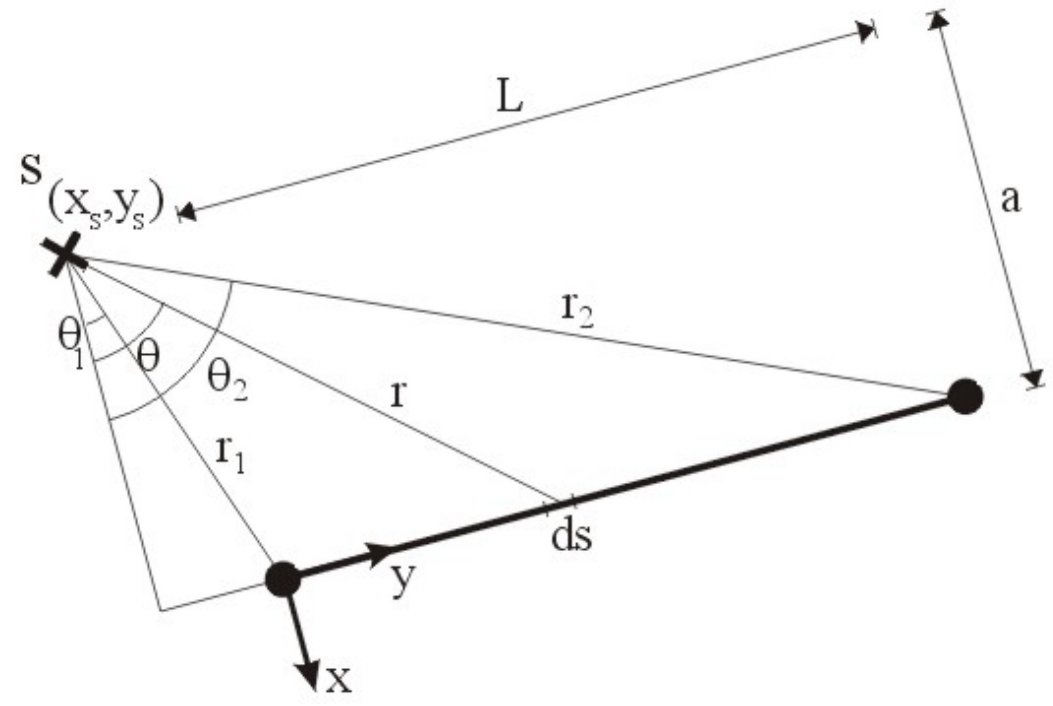

Figura D1 - Ponto fonte não alinhado com o elemento

\section{Matriz H:}

$$
\begin{aligned}
H_{11}^{\prime}= & K_{3}\left\{K_{4}\left[\operatorname{Ln}\left(\operatorname{Cos}\left(\theta_{2}\right)\right) \frac{a}{L}+\theta_{2} \phi_{1}\right]+\left(\operatorname{Sen}\left(\theta_{2}\right) \operatorname{Cos}\left(\theta_{2}\right)+\theta_{2}\right) \phi_{1}+\operatorname{Cos}\left(\theta_{2}\right)^{2} \frac{a}{L}\right\}+ \\
& -K_{3}\left\{K_{4}\left[\operatorname{Ln}\left(\operatorname{Cos}\left(\theta_{1}\right)\right) \frac{a}{L}+\theta_{1} \phi_{1}\right]+\left(\operatorname{Sen}\left(\theta_{1}\right) \operatorname{Cos}\left(\theta_{1}\right)+\theta_{1}\right) \phi_{1}+\operatorname{Cos}\left(\theta_{1}\right)^{2} \frac{a}{L}\right\} \\
H_{12}^{1}= & K_{3}\left\{K_{4}\left[-\operatorname{Ln}\left(\operatorname{Cos}\left(\theta_{2}\right)\right) \phi_{1}+\left(-\operatorname{Tg}\left(\theta_{2}\right)+\theta_{2}\right) \frac{a}{L}\right]-\left(-\operatorname{Sen}\left(\theta_{2}\right) \operatorname{Cos}\left(\theta_{2}\right)+\theta_{2}\right) \frac{a}{L}+\right. \\
& \left.-\operatorname{Cos}\left(\theta_{2}\right)^{2} \phi_{1}\right\}-K_{3}\left\{K_{4}\left[-\operatorname{Ln}\left(\operatorname{Cos}\left(\theta_{1}\right)\right) \phi_{1}+\left(-\operatorname{Tg}\left(\theta_{1}\right)+\theta_{1}\right) \frac{a}{L}\right]+\right. \\
& \left.-\left(-\operatorname{Sen}\left(\theta_{1}\right) \operatorname{Cos}\left(\theta_{1}\right)+\theta_{1}\right) \frac{a}{L}-\operatorname{Cos}\left(\theta_{1}\right)^{2} \phi_{1}\right\}
\end{aligned}
$$




$$
\begin{aligned}
& H_{21}^{1}=K_{3}\left\{K_{4}\left[\operatorname{Ln}\left(\operatorname{Cos}\left(\theta_{2}\right)\right) \phi_{1}-\left(-\operatorname{Tg}\left(\theta_{2}\right)+\theta_{2}\right) \frac{a}{L}\right]-\left(-\operatorname{Sen}\left(\theta_{2}\right) \operatorname{Cos}\left(\theta_{2}\right)+\theta_{2}\right) \frac{a}{L}+\right. \\
& \left.-\operatorname{Cos}\left(\theta_{2}\right)^{2} \phi_{1}\right\}-\mathrm{K}_{3}\left\{\mathrm{~K}_{4}\left[\operatorname{Ln}\left(\operatorname{Cos}\left(\theta_{1}\right)\right) \phi_{1}-\left(-\operatorname{Tg}\left(\theta_{1}\right)+\theta_{1}\right) \frac{\mathrm{a}}{\mathrm{L}}\right]+\right. \\
& \left.-\left(-\operatorname{Sen}\left(\theta_{1}\right) \operatorname{Cos}\left(\theta_{1}\right)+\theta_{1}\right) \frac{a}{L}-\operatorname{Cos}\left(\theta_{1}\right)^{2} \phi_{1}\right\} \\
& \mathrm{H}_{22}^{1}=\mathrm{K}_{3}\left\{\mathrm{~K}_{4}\left[\operatorname{Ln}\left(\operatorname{Cos}\left(\theta_{2}\right)\right) \frac{\mathrm{a}}{\mathrm{L}}+\theta_{2} \phi_{1}\right]+\left(-\operatorname{Sen}\left(\theta_{2}\right) \operatorname{Cos}\left(\theta_{2}\right)+\theta_{2}\right) \phi_{1}+\operatorname{Sen}\left(\theta_{2}\right)^{2} \frac{\mathrm{a}}{\mathrm{L}}+\right. \\
& \left.+\frac{2 \mathrm{aLn}\left(\operatorname{Cos}\left(\theta_{2}\right)\right)}{\mathrm{L}}\right\}-\mathrm{K}_{3}\left\{\mathrm{~K}_{4}\left[\operatorname{Ln}\left(\operatorname{Cos}\left(\theta_{1}\right)\right) \frac{\mathrm{a}}{\mathrm{L}}+\theta_{1} \phi_{1}\right]+\left(-\operatorname{Sen}\left(\theta_{1}\right) \operatorname{Cos}\left(\theta_{1}\right)+\theta_{1}\right) \phi_{1}+\right. \\
& \left.+\operatorname{Sen}\left(\theta_{1}\right)^{2} \frac{a}{L}+\frac{2 a \operatorname{Ln}\left(\operatorname{Cos}\left(\theta_{1}\right)\right)}{\mathrm{L}}\right\} \\
& H_{11}^{2}=K_{3}\left\{K_{4}\left[-\operatorname{Ln}\left(\operatorname{Cos}\left(\theta_{2}\right)\right) \frac{a}{L}+\theta_{2} \phi_{2}\right]+\left(\operatorname{Sen}\left(\theta_{2}\right) \operatorname{Cos}\left(\theta_{2}\right)+\theta_{2}\right) \phi_{2}-\operatorname{Cos}\left(\theta_{2}\right)^{2} \frac{a}{L}\right\}+ \\
& -\mathrm{K}_{3}\left\{\mathrm{~K}_{4}\left[-\mathrm{Ln}\left(\operatorname{Cos}\left(\theta_{1}\right)\right) \frac{\mathrm{a}}{\mathrm{L}}+\theta_{1} \phi_{2}\right]+\left(\operatorname{Sen}\left(\theta_{1}\right) \operatorname{Cos}\left(\theta_{1}\right)+\theta_{1}\right) \phi_{2}-\operatorname{Cos}\left(\theta_{1}\right)^{2} \frac{\mathrm{a}}{\mathrm{L}}\right\} \\
& H_{12}^{2}=K_{3}\left\{K_{4}\left[-\operatorname{Ln}\left(\operatorname{Cos}\left(\theta_{2}\right)\right) \phi_{2}-\left(-\operatorname{Tg}\left(\theta_{2}\right)+\theta_{2}\right) \frac{a}{L}\right]+\left(-\operatorname{Sen}\left(\theta_{2}\right) \operatorname{Cos}\left(\theta_{2}\right)+\theta_{2}\right) \frac{a}{L}+\right. \\
& \left.-\operatorname{Cos}\left(\theta_{2}\right)^{2} \phi_{2}\right\}-\mathrm{K}_{3}\left\{\mathrm{~K}_{4}\left[-\operatorname{Ln}\left(\operatorname{Cos}\left(\theta_{1}\right)\right) \phi_{2}-\left(-\operatorname{Tg}\left(\theta_{1}\right)+\theta_{1}\right) \frac{\mathrm{a}}{\mathrm{L}}\right]+\right. \\
& \left.+\left(-\operatorname{Sen}\left(\theta_{1}\right) \operatorname{Cos}\left(\theta_{1}\right)+\theta_{1}\right) \frac{\mathrm{a}}{\mathrm{L}}-\operatorname{Cos}\left(\theta_{1}\right)^{2} \phi_{2}\right\} \\
& \mathrm{H}_{21}^{2}=\mathrm{K}_{3}\left\{\mathrm{~K}_{4}\left[\operatorname{Ln}\left(\operatorname{Cos}\left(\theta_{2}\right)\right) \phi_{2}+\left(-\operatorname{Tg}\left(\theta_{2}\right)+\theta_{2}\right) \frac{\mathrm{a}}{\mathrm{L}}\right]+\left(-\operatorname{Sen}\left(\theta_{2}\right) \operatorname{Cos}\left(\theta_{2}\right)+\theta_{2}\right) \frac{\mathrm{a}}{\mathrm{L}}+\right. \\
& \left.-\operatorname{Cos}\left(\theta_{2}\right)^{2} \phi_{2}\right\}-K_{3}\left\{K_{4}\left[\operatorname{Ln}\left(\operatorname{Cos}\left(\theta_{1}\right)\right) \phi_{2}+\left(-\operatorname{Tg}\left(\theta_{1}\right)+\theta_{1}\right) \frac{a}{L}\right]+\right. \\
& \left.+\left(-\operatorname{Sen}\left(\theta_{1}\right) \operatorname{Cos}\left(\theta_{1}\right)+\theta_{1}\right) \frac{\mathrm{a}}{\mathrm{L}}-\operatorname{Cos}\left(\theta_{1}\right)^{2} \phi_{2}\right\}
\end{aligned}
$$




$$
\begin{aligned}
\mathrm{H}_{22}^{2}= & \mathrm{K}_{3}\left\{\mathrm{~K}_{4}\left[-\operatorname{Ln}\left(\operatorname{Cos}\left(\theta_{2}\right)\right) \frac{\mathrm{a}}{\mathrm{L}}+\theta_{2} \phi_{2}\right]+\left(-\operatorname{Sen}\left(\theta_{2}\right) \operatorname{Cos}\left(\theta_{2}\right)+\theta_{2}\right) \phi_{2}-\operatorname{Sen}\left(\theta_{2}\right)^{2} \frac{\mathrm{a}}{\mathrm{L}}+\right. \\
& \left.-\frac{2 \mathrm{aLn}\left(\operatorname{Cos}\left(\theta_{2}\right)\right)}{\mathrm{L}}\right\}-\mathrm{K}_{3}\left\{\mathrm{~K}_{4}\left[-\operatorname{Ln}\left(\operatorname{Cos}\left(\theta_{1}\right)\right) \frac{\mathrm{a}}{\mathrm{L}}+\theta_{1} \phi_{2}\right]+\left(-\operatorname{Sen}\left(\theta_{1}\right) \operatorname{Cos}\left(\theta_{1}\right)+\theta_{1}\right) \phi_{2}+\right. \\
& \left.-\operatorname{Sen}\left(\theta_{1}\right)^{2} \frac{\mathrm{a}}{\mathrm{L}}-\frac{2 \mathrm{aLn}\left(\operatorname{Cos}\left(\theta_{1}\right)\right)}{\mathrm{L}}\right\}
\end{aligned}
$$

\section{Matriz G:}

$$
\begin{aligned}
& \mathrm{G}_{11}^{1}=\mathrm{K}_{1}\left\{\mathrm { K } _ { 2 } \left[\operatorname{Ln}\left(\frac{\mathrm{a}}{\operatorname{Cos}\left(\theta_{2}\right)}\right)\left[\operatorname{Tg}\left(\theta_{2}\right) \phi_{1}-\frac{\mathrm{a}}{2 \mathrm{LCOS}\left(\theta_{2}\right)^{2}}\right]+\left(-\operatorname{Tg}\left(\theta_{2}\right)+\theta_{2}\right) \phi_{1}+\right.\right. \\
& \left.\left.+\frac{\mathrm{a}}{4 \mathrm{~L} \operatorname{Cos}\left(\theta_{2}\right)^{2}}\right]-\theta_{2} \phi_{1}-\frac{\mathrm{a}}{\mathrm{L}} \mathrm{Ln}\left(\operatorname{Cos}\left(\theta_{2}\right)\right)\right\}-\mathrm{K}_{1}\left\{\mathrm { K } _ { 2 } \left[\operatorname { L n } ( \frac { \mathrm { a } } { \operatorname { C o s } ( \theta _ { 1 } ) } ) \left[\operatorname{Tg}\left(\theta_{1}\right) \phi_{1}+\right.\right.\right. \\
& \left.\left.\left.-\frac{\mathrm{a}}{2 \mathrm{~L} \operatorname{Cos}\left(\theta_{1}\right)^{2}}\right]+\left(-\operatorname{Tg}\left(\theta_{1}\right)+\theta_{1}\right) \phi_{1}+\frac{\mathrm{a}}{4 \mathrm{~L} \operatorname{Cos}\left(\theta_{1}\right)^{2}}\right]-\theta_{1} \phi_{1}-\frac{\mathrm{a}}{\mathrm{L}} \mathrm{Ln}\left(\operatorname{Cos}\left(\theta_{1}\right)\right)\right\} \\
& \mathrm{G}_{12}^{1}=\mathrm{K}_{1}\left\{\operatorname{Ln}\left(\operatorname{Cos}\left(\theta_{2}\right)\right) \phi_{1}+\frac{\left(\operatorname{Tg}\left(\theta_{2}\right)-\theta_{2}\right)}{\mathrm{L}}\right\}-\mathrm{K}_{1}\left\{\operatorname{Ln}\left(\operatorname{Cos}\left(\theta_{1}\right)\right) \phi_{1}+\frac{\left(\operatorname{Tg}\left(\theta_{1}\right)-\theta_{1}\right)}{\mathrm{L}}\right\} \\
& \mathrm{G}_{21}^{1}=\mathrm{G}_{12}^{1} \\
& \mathrm{G}_{22}^{1}=\mathrm{K}_{1}\left\{\mathrm { K } _ { 2 } \left[\operatorname{Ln}\left(\frac{\mathrm{a}}{\operatorname{Cos}\left(\theta_{2}\right)}\right)\left[\operatorname{Tg}\left(\theta_{2}\right) \phi_{1}-\frac{\mathrm{a}}{2 \operatorname{LCOS}\left(\theta_{2}\right)^{2}}\right]+\left(-\operatorname{Tg}\left(\theta_{2}\right)+\theta_{2}\right) \phi_{1}+\right.\right. \\
& \left.\left.+\frac{\mathrm{a}}{4 \mathrm{~L} \operatorname{Cos}\left(\theta_{2}\right)^{2}}\right]+\left(-\operatorname{Tg}\left(\theta_{2}\right)+\theta_{2}\right) \phi_{1}+\frac{\mathrm{aTg}\left(\theta_{2}\right)^{2}}{2 \mathrm{~L}}+\frac{\mathrm{aLn}\left(\operatorname{Cos}\left(\theta_{2}\right)\right)}{\mathrm{L}}\right\}+ \\
& -\mathrm{K}_{1}\left\{\mathrm { K } _ { 2 } \left[\operatorname{Ln}\left(\frac{\mathrm{a}}{\operatorname{Cos}\left(\theta_{1}\right)}\right)\left[\operatorname{Tg}\left(\theta_{1}\right) \phi_{1}-\frac{a}{2 \mathrm{LCos}\left(\theta_{1}\right)^{2}}\right]+\left(-\operatorname{Tg}\left(\theta_{1}\right)+\theta_{1}\right) \phi_{1}+\right.\right. \\
& \left.\left.+\frac{\mathrm{a}}{4 \mathrm{~L} \operatorname{Cos}\left(\theta_{1}\right)^{2}}\right]+\left(-\operatorname{Tg}\left(\theta_{1}\right)+\theta_{1}\right) \phi_{1}+\frac{\mathrm{aTg}\left(\theta_{1}\right)^{2}}{2 \mathrm{~L}}+\frac{\mathrm{aLn}\left(\operatorname{Cos}\left(\theta_{1}\right)\right)}{\mathrm{L}}\right\}
\end{aligned}
$$




$$
\begin{aligned}
& \mathrm{G}_{11}^{2}=\mathrm{K}_{1}\left\{\mathrm { K } _ { 2 } \left[\operatorname{Ln}\left(\frac{\mathrm{a}}{\operatorname{Cos}\left(\theta_{2}\right)}\right)\left[\operatorname{Tg}\left(\theta_{2}\right) \phi_{2}+\frac{\mathrm{a}}{2 \mathrm{LCos}\left(\theta_{2}\right)^{2}}\right]+\left(-\operatorname{Tg}\left(\theta_{2}\right)+\theta_{2}\right) \phi_{2}+\right.\right. \\
& \left.\left.-\frac{\mathrm{a}}{4 \mathrm{~L} \operatorname{Cos}\left(\theta_{2}\right)^{2}}\right]-\theta_{2} \phi_{2}+\frac{\mathrm{a}}{\mathrm{L}} \operatorname{Ln}\left(\operatorname{Cos}\left(\theta_{2}\right)\right)\right\}-\mathrm{K}_{1}\left\{\mathrm { K } _ { 2 } \left[\operatorname { L n } ( \frac { \mathrm { a } } { \operatorname { C o s } ( \theta _ { 1 } ) } ) \left[\operatorname{Tg}\left(\theta_{1}\right) \phi_{2}+\right.\right.\right. \\
& \left.\left.\left.+\frac{\mathrm{a}}{2 \mathrm{~L} \operatorname{Cos}\left(\theta_{1}\right)^{2}}\right]+\left(-\operatorname{Tg}\left(\theta_{1}\right)+\theta_{1}\right) \phi_{2}-\frac{\mathrm{a}}{4 \operatorname{LCos}\left(\theta_{1}\right)^{2}}\right]-\theta_{1} \phi_{2}+\frac{\mathrm{a}}{\mathrm{L}} \operatorname{Ln}\left(\operatorname{Cos}\left(\theta_{1}\right)\right)\right\} \\
& \mathrm{G}_{12}^{2}=\mathrm{K}_{1}\left\{\operatorname{Ln}\left(\operatorname{Cos}\left(\theta_{2}\right)\right) \phi_{2}-\frac{\left(\operatorname{Tg}\left(\theta_{2}\right)-\theta_{2}\right)}{\mathrm{L}}\right\}-\mathrm{K}_{1}\left\{\operatorname{Ln}\left(\operatorname{Cos}\left(\theta_{1}\right)\right) \phi_{2}-\frac{\left(\operatorname{Tg}\left(\theta_{1}\right)-\theta_{1}\right)}{\mathrm{L}}\right\} \\
& \mathrm{G}_{21}^{2}=\mathrm{G}_{12}^{2} \\
& \mathrm{G}_{22}^{2}=\mathrm{K}_{1}\left\{\mathrm { K } _ { 2 } \left[\operatorname{Ln}\left(\frac{\mathrm{a}}{\operatorname{Cos}\left(\theta_{2}\right)}\right)\left[\operatorname{Tg}\left(\theta_{2}\right) \phi_{2}+\frac{\mathrm{a}}{2 \mathrm{LCOS}\left(\theta_{2}\right)^{2}}\right]+\left(-\operatorname{Tg}\left(\theta_{2}\right)+\theta_{2}\right) \phi_{2}+\right.\right. \\
& \left.\left.-\frac{\mathrm{a}}{4 \mathrm{~L} \operatorname{Cos}\left(\theta_{2}\right)^{2}}\right]+\left(-\operatorname{Tg}\left(\theta_{2}\right)+\theta_{2}\right) \phi_{2}-\frac{\operatorname{aTg}\left(\theta_{2}\right)^{2}}{2 \mathrm{~L}}-\frac{\mathrm{aLn}\left(\operatorname{Cos}\left(\theta_{2}\right)\right)}{\mathrm{L}}\right\}+ \\
& -\mathrm{K}_{1}\left\{\mathrm { K } _ { 2 } \left[\operatorname{Ln}\left(\frac{\mathrm{a}}{\operatorname{Cos}\left(\theta_{1}\right)}\right)\left[\operatorname{Tg}\left(\theta_{1}\right) \phi_{2}+\frac{\mathrm{a}}{2 \mathrm{LCos}\left(\theta_{1}\right)^{2}}\right]+\left(-\operatorname{Tg}\left(\theta_{1}\right)+\theta_{1}\right) \phi_{2}+\right.\right. \\
& \left.\left.-\frac{\mathrm{a}}{4 \mathrm{~L} \operatorname{Cos}\left(\theta_{1}\right)^{2}}\right]+\left(-\operatorname{Tg}\left(\theta_{1}\right)+\theta_{1}\right) \phi_{2}-\frac{\operatorname{aTg}\left(\theta_{1}\right)^{2}}{2 \mathrm{~L}}-\frac{\operatorname{aLn}\left(\operatorname{Cos}\left(\theta_{1}\right)\right)}{\mathrm{L}}\right\}
\end{aligned}
$$

\section{Matriz S:}

$$
\begin{aligned}
\mathrm{S}_{11}^{1}= & \frac{\mathrm{K}_{7}}{\mathrm{a}}\left\{\left[\left(2 \mathrm{~K}_{4}+4 v\right)\left(\operatorname{Sen}\left(\theta_{2}\right) \operatorname{Cos}\left(\theta_{2}\right)+\theta_{2}\right)-\left(2 \operatorname{Sen}\left(\theta_{2}\right) \operatorname{Cos}\left(\theta_{2}\right)^{3}+\right.\right.\right. \\
& \left.\left.+3 \operatorname{Sen}\left(\theta_{2}\right) \operatorname{Cos}\left(\theta_{2}\right)+3 \theta_{2}\right)+\left(2 \mathrm{~K}_{4}-\mathrm{K}_{5}\right) \theta_{2}\right] \phi_{1}-\frac{\mathrm{a}}{\mathrm{L}}\left[-\left(2 \mathrm{~K}_{4}+4 v\right) \operatorname{Cos}\left(\theta_{2}\right)^{2}+\right. \\
& \left.\left.+2 \operatorname{Cos}\left(\theta_{2}\right)^{4}-\left(2 \mathrm{~K}_{4}-\mathrm{K}_{5}\right) \operatorname{Ln}\left(\operatorname{Cos}\left(\theta_{2}\right)\right)\right]\right\}-\frac{\mathrm{K}_{7}}{\mathrm{a}}\left\{\left[\left(2 \mathrm{~K}_{4}+4 v\right)\left(\operatorname{Sen}\left(\theta_{1}\right) \operatorname{Cos}\left(\theta_{1}\right)+\theta_{1}\right)+\right.\right. \\
& \left.-\left(2 \operatorname{Sen}\left(\theta_{1}\right) \operatorname{Cos}\left(\theta_{1}\right)^{3}+3 \operatorname{Sen}\left(\theta_{1}\right) \operatorname{Cos}\left(\theta_{1}\right)+3 \theta_{1}\right)+\left(2 \mathrm{~K}_{4}-\mathrm{K}_{5}\right) \theta_{1}\right] \phi_{1}+ \\
& \left.-\frac{\mathrm{a}}{\mathrm{L}}\left[-\left(2 \mathrm{~K}_{4}+4 v\right) \operatorname{Cos}\left(\theta_{1}\right)^{2}+2 \operatorname{Cos}\left(\theta_{1}\right)^{4}-\left(2 \mathrm{~K}_{4}-\mathrm{K}_{5}\right) \operatorname{Ln}\left(\operatorname{Cos}\left(\theta_{1}\right)\right)\right]\right\}
\end{aligned}
$$




$$
\begin{aligned}
S_{12}^{1}= & \frac{K_{7}}{a}\left\{\left[-\left(K_{4}+2 v\right) \operatorname{Cos}\left(\theta_{2}\right)^{2}+2 \operatorname{Cos}\left(\theta_{2}\right)^{4}\right] \phi_{1}-\frac{a}{L}\left[( \mathbf { K } _ { 1 } + 2 v ) \left(-\operatorname{Sen}\left(\theta_{2}\right) \operatorname{Cos}\left(\theta_{2}\right)+\right.\right.\right. \\
& \left.\left.\left.+\theta_{2}\right)-\left(-2 \operatorname{Sen}\left(\theta_{2}\right) \operatorname{Cos}\left(\theta_{2}\right)^{3}+\operatorname{Sen}\left(\theta_{2}\right) \operatorname{Cos}\left(\theta_{2}\right)+\theta_{2}\right)\right]\right\}+ \\
& -\frac{K_{7}}{a}\left\{\left[-\left(K_{4}+2 v\right) \operatorname{Cos}\left(\theta_{1}\right)^{2}+2 \operatorname{Cos}\left(\theta_{1}\right)^{4}\right] \phi_{1}-\frac{a}{L}\left[( K _ { 4 } + 2 v ) \left(-\operatorname{Sen}\left(\theta_{1}\right) \operatorname{Cos}\left(\theta_{1}\right)+\right.\right.\right. \\
& \left.\left.\left.+\theta_{1}\right)-\left(-2 \operatorname{Sen}\left(\theta_{1}\right) \operatorname{Cos}\left(\theta_{1}\right)^{3}+\operatorname{Sen}\left(\theta_{1}\right) \operatorname{Cos}\left(\theta_{1}\right)+\theta_{1}\right)\right]\right\} \\
S_{21}^{1}= & S_{12}^{1} \\
S_{22}^{1}= & \frac{K_{7}}{a}\left\{\left[\left(K_{4}+2 v\right) \theta_{2}-\left(-2 \operatorname{Sen}\left(\theta_{2}\right) \operatorname{Cos}\left(\theta_{2}\right)^{3}+\operatorname{Sen}\left(\theta_{2}\right) \operatorname{Cos}\left(\theta_{2}\right)+\theta_{2}\right)\right] \phi_{1}+\right. \\
& \left.-\frac{a}{L}\left[-v\left(\operatorname{Sen}\left(\theta_{2}\right)^{2}+\operatorname{Cos}\left(\theta_{2}\right)^{2}+2 \operatorname{Ln}\left(\operatorname{Cos}\left(\theta_{2}\right)\right)\right)-2 \operatorname{Sen}\left(\theta_{2}\right)^{4}-K_{4} \operatorname{Ln}\left(\operatorname{Cos}\left(\theta_{2}\right)\right)\right]\right\}+ \\
& -\frac{K_{7}}{a}\left\{\left[\left(K_{4}+2 v\right) \theta_{1}-\left(-2 \operatorname{Sen}\left(\theta_{1}\right) \operatorname{Cos}\left(\theta_{1}\right)^{3}+\operatorname{Sen}\left(\theta_{1}\right) \operatorname{Cos}\left(\theta_{1}\right)+\theta_{1}\right)\right] \phi_{1}+\right. \\
& \left.-\frac{a}{L}\left[-v\left(\operatorname{Sen}\left(\theta_{1}\right)^{2}+\operatorname{Cos}\left(\theta_{1}\right)^{2}+2 \operatorname{Ln}\left(\operatorname{Cos}\left(\theta_{1}\right)\right)\right)-2 \operatorname{Sen}\left(\theta_{1}\right)^{4}-K_{4} \operatorname{Ln}\left(\operatorname{Cos}\left(\theta_{1}\right)\right)\right]\right\} \\
& \left.\left.\left.+\theta_{1}\right)-\left(-2 \operatorname{Sen}\left(\theta_{1}\right)^{3} \operatorname{Cos}\left(\theta_{1}\right)-3 \operatorname{Sen}\left(\theta_{1}\right) \operatorname{Cos}\left(\theta_{1}\right)+3 \theta_{1}\right)\right]\right\} \\
S_{32}^{1}= & \frac{K_{7}}{a}\left\{\left[-\left(K_{4}+2 v\right) \operatorname{Cos}\left(\theta_{2}\right)^{2}-2 \operatorname{Sen}\left(\theta_{2}\right)^{4}\right] \phi_{1}-\frac{a}{L}\left[( K _ { 4 } + 2 v ) \left(-\operatorname{Sen}\left(\theta_{2}\right) \operatorname{Cos}\left(\theta_{2}\right)+\right.\right.\right. \\
& \left.\left.\left.+\theta_{2}\right)-\left(-2 \operatorname{Sen}\left(\theta_{2}\right)^{3} \operatorname{Cos}\left(\theta_{2}\right)-3 \operatorname{Sen}\left(\theta_{2}\right) \operatorname{Cos}\left(\theta_{2}\right)+3 \theta_{2}\right)\right]\right\}+ \\
& -\frac{K_{7}}{a}\left\{\left[-\left(K_{4}+2 v\right) \operatorname{Cos}\left(\theta_{1}\right)^{2}-2 \operatorname{Sen}\left(\theta_{1}\right)^{4}\right] \phi_{1}-\frac{a}{L}\left[( K _ { 4 } + 2 v ) \left(-\operatorname{Sen}\left(\theta_{1}\right) \operatorname{Cos}\left(\theta_{1}\right)+\right.\right.\right. \\
S_{31}^{1}= & \frac{K_{7}}{a}\left\{\left[\left(2 K_{4}-K_{5}\right) \theta_{2}-\left(-2 \operatorname{Sen}\left(\theta_{2}\right) \operatorname{Cos}\left(\theta_{2}\right)^{3}+\operatorname{Sen}\left(\theta_{2}\right) \operatorname{Cos}\left(\theta_{2}\right)+\theta_{2}\right)\right] \phi_{1}+\right. \\
& \left.-\frac{a}{L}\left[-2 \operatorname{Sen}\left(\theta_{2}\right)^{4}-\left(2 K_{4}-K_{5}\right) \operatorname{Ln}\left(\operatorname{Cos}\left(\theta_{2}\right)\right)\right]\right\} \\
& -\frac{K_{7}}{a}\left\{\left[\left(2 K_{4}-K_{5}\right) \theta_{1}-\left(-2 \operatorname{Sen}\left(\theta_{1}\right) \operatorname{Cos}\left(\theta_{1}\right)^{3}+\operatorname{Sen}\left(\theta_{1}\right) \operatorname{Cos}\left(\theta_{1}\right)+\theta_{1}\right)\right] \phi_{1}\right. \\
& \left.-\frac{a}{L}\left[-2 \operatorname{Sen}\left(\theta_{1}\right)^{4}-\left(2 K_{4}-K_{5}\right) \operatorname{Ln}\left(\operatorname{Cos}\left(\theta_{1}\right)\right)\right]\right\} \\
&
\end{aligned}
$$




$$
\begin{aligned}
S_{11}^{2}= & \frac{K_{7}}{a}\left\{\left[\left(2 K_{4}+4 v\right)\left(\operatorname{Sen}\left(\theta_{2}\right) \operatorname{Cos}\left(\theta_{2}\right)+\theta_{2}\right)-\left(2 \operatorname{Sen}\left(\theta_{2}\right) \operatorname{Cos}\left(\theta_{2}\right)^{3}+\right.\right.\right. \\
& \left.\left.+3 \operatorname{Sen}\left(\theta_{2}\right) \operatorname{Cos}\left(\theta_{2}\right)+3 \theta_{2}\right)+\left(2 K_{4}-K_{5}\right) \theta_{2}\right] \phi_{2}+\frac{a}{L}\left[-\left(2 K_{4}+4 v\right) \operatorname{Cos}\left(\theta_{2}\right)^{2}+\right. \\
& \left.\left.+2 \operatorname{Cos}\left(\theta_{2}\right)^{4}-\left(2 K_{4}-K_{5}\right) \operatorname{Ln}\left(\operatorname{Cos}\left(\theta_{2}\right)\right)\right]\right\}-\frac{K_{7}}{a}\left\{\left[\left(2 K_{4}+4 v\right)\left(\operatorname{Sen}\left(\theta_{1}\right) \operatorname{Cos}\left(\theta_{1}\right)+\theta_{1}\right)+\right.\right. \\
& \left.-\left(2 \operatorname{Sen}\left(\theta_{1}\right) \operatorname{Cos}\left(\theta_{1}\right)^{3}+3 \operatorname{Sen}\left(\theta_{1}\right) \operatorname{Cos}\left(\theta_{1}\right)+3 \theta_{1}\right)+\left(2 K_{4}-K_{5}\right) \theta_{1}\right] \phi_{2}+ \\
& \left.+\frac{a}{L}\left[-\left(2 K_{4}+4 v\right) \operatorname{Cos}\left(\theta_{1}\right)^{2}+2 \operatorname{Cos}\left(\theta_{1}\right)^{4}-\left(2 K_{4}-K_{5}\right) \operatorname{Ln}\left(\operatorname{Cos}\left(\theta_{1}\right)\right)\right]\right\} \\
S_{12}^{2}= & \frac{K_{7}}{a}\left\{\left[-\left(K_{4}+2 v\right) \operatorname{Cos}\left(\theta_{2}\right)^{2}+2 \operatorname{Cos}\left(\theta_{2}\right)^{4}\right] \phi_{2}+\frac{a}{L}\left[( K _ { 4 } + 2 v ) \left(-\operatorname{Sen}\left(\theta_{2}\right) \operatorname{Cos}\left(\theta_{2}\right)+\right.\right.\right. \\
& \left.\left.\left.+\theta_{2}\right)-\left(-2 \operatorname{Sen}\left(\theta_{2}\right) \operatorname{Cos}\left(\theta_{2}\right)^{3}+\operatorname{Sen}\left(\theta_{2}\right) \operatorname{Cos}\left(\theta_{2}\right)+\theta_{2}\right)\right]\right\}+ \\
& -\frac{K_{7}}{a}\left\{\left[-\left(K_{4}+2 v\right) \operatorname{Cos}\left(\theta_{1}\right)^{2}+2 \operatorname{Cos}\left(\theta_{1}\right)^{4}\right] \phi_{2}+\frac{a}{L}\left[( K _ { 4 } + 2 v ) \left(-\operatorname{Sen}\left(\theta_{1}\right) \operatorname{Cos}\left(\theta_{1}\right)+\right.\right.\right. \\
& \left.\left.\left.+\theta_{1}\right)-\left(-2 \operatorname{Sen}\left(\theta_{1}\right) \operatorname{Cos}\left(\theta_{1}\right)^{3}+\operatorname{Sen}\left(\theta_{1}\right) \operatorname{Cos}\left(\theta_{1}\right)+\theta_{1}\right)\right]\right\} \\
& \left.+\frac{a}{L}\left[-2 \operatorname{Sen}\left(\theta_{1}\right)^{4}-\left(2 K_{4}-K_{5}\right) \operatorname{Ln}\left(\operatorname{Cos}\left(\theta_{1}\right)\right)\right]\right\} \\
& -\frac{K_{7}}{a}\left\{\left[\left(2 K_{4}-K_{5}\right) \theta_{1}-\left(-2 \operatorname{Sen}\left(\theta_{1}\right) \operatorname{Cos}\left(\theta_{1}\right)^{3}+\operatorname{Sen}\left(\theta_{1}\right) \operatorname{Cos}\left(\theta_{1}\right)+\theta_{1}\right)\right] \phi_{2}\right. \\
S_{21}^{2}= & S_{12}^{2} \\
& \left.+\frac{a}{L}\left[-v\left(\operatorname{Sen}\left(\theta_{1}\right)^{2}+\operatorname{Cos}\left(\theta_{1}\right)^{2}+2 \operatorname{Ln}\left(\operatorname{Cos}\left(\theta_{1}\right)\right)\right)-2 \operatorname{Sen}\left(\theta_{1}\right)^{4}-K_{4} \operatorname{Ln}\left(\operatorname{Cos}\left(\theta_{1}\right)\right)\right]\right\} \\
& +\frac{K_{7}}{a}\left\{\left[\left(2 K_{4}-K_{5}\right) \theta_{2}-\left(-2 \operatorname{Sen}\left(\theta_{2}\right) \operatorname{Cos}\left(\theta_{2}\right)^{3}+\operatorname{Sen}\left(\theta_{2}\right) \operatorname{Cos}\left(\theta_{2}\right)+\theta_{2}\right)\right] \phi_{2}+\right. \\
& +\frac{K_{7}}{a}\left\{\left[\left(K_{4}+2 v\right) \theta_{2}-\left(-2 \operatorname{Sen}\left(\theta_{2}\right) \operatorname{Cos}\left(\theta_{2}\right)^{3}+\operatorname{Sen}\left(\theta_{2}\right) \operatorname{Cos}\left(\theta_{2}\right)+\theta_{2}\right)\right] \phi_{2}+\right. \\
& \left.+\frac{a}{L}\left[-v\left(\operatorname{Sen}\left(\theta_{2}\right)^{2}+\operatorname{Cos}\left(\theta_{2}\right)^{2}+2 \operatorname{Ln}\left(\operatorname{Cos}\left(\theta_{2}\right)\right)\right)-2 \operatorname{Sen}\left(\theta_{2}\right)^{4}-K_{4} \operatorname{Ln}\left(\operatorname{Cos}\left(\theta_{2}\right)\right)\right]\right\}+ \\
& -\frac{K_{7}}{a}\left\{\left[\left(K_{4}+2 v\right) \theta_{1}-\left(-2 \operatorname{Sen}\left(\theta_{1}\right) \operatorname{Cos}\left(\theta_{1}\right)^{3}+\operatorname{Sen}\left(\theta_{1}\right) \operatorname{Cos}\left(\theta_{1}\right)+\theta_{1}\right)\right] \phi_{2}+\right. \\
& \\
&
\end{aligned}
$$




$$
\begin{aligned}
S_{32}^{2} & =\frac{K_{7}}{a}\left\{\left[-\left(K_{4}+2 v\right) \operatorname{Cos}\left(\theta_{2}\right)^{2}-2 \operatorname{Sen}\left(\theta_{2}\right)^{4}\right] \phi_{2}+\frac{a}{L}\left[( K _ { 4 } + 2 v ) \left(-\operatorname{Sen}\left(\theta_{2}\right) \operatorname{Cos}\left(\theta_{2}\right)+\right.\right.\right. \\
& \left.\left.\left.+\theta_{2}\right)-\left(-2 \operatorname{Sen}\left(\theta_{2}\right)^{3} \operatorname{Cos}\left(\theta_{2}\right)-3 \operatorname{Sen}\left(\theta_{2}\right) \operatorname{Cos}\left(\theta_{2}\right)+3 \theta_{2}\right)\right]\right\}+ \\
& -\frac{K_{7}}{a}\left\{\left[-\left(K_{4}+2 v\right) \operatorname{Cos}\left(\theta_{1}\right)^{2}-2 \operatorname{Sen}\left(\theta_{1}\right)^{4}\right] \phi_{2}+\frac{a}{L}\left[( K _ { 4 } + 2 v ) \left(-\operatorname{Sen}\left(\theta_{1}\right) \operatorname{Cos}\left(\theta_{1}\right)+\right.\right.\right. \\
& \left.\left.\left.+\theta_{1}\right)-\left(-2 \operatorname{Sen}\left(\theta_{1}\right)^{3} \operatorname{Cos}\left(\theta_{1}\right)-3 \operatorname{Sen}\left(\theta_{1}\right) \operatorname{Cos}\left(\theta_{1}\right)+3 \theta_{1}\right)\right]\right\}
\end{aligned}
$$

\section{Matriz D:}

$$
\begin{aligned}
\mathbf{D}_{11}^{1}= & -\mathrm{K}_{3}\left\{\left[\left(\mathrm{~K}_{4}+1\right) \theta_{2}+\operatorname{Sen}\left(\theta_{2}\right) \operatorname{Cos}\left(\theta_{2}\right)\right] \phi_{1}-\frac{\mathrm{a}}{\mathrm{L}}\left[-\mathrm{K}_{4} \operatorname{Ln}\left(\operatorname{Cos}\left(\theta_{2}\right)\right)-\operatorname{Cos}\left(\theta_{2}\right)^{2}\right]\right\}+ \\
& +\mathrm{K}_{3}\left\{\left[\left(\mathrm{~K}_{4}+1\right) \theta_{1}+\operatorname{Sen}\left(\theta_{1}\right) \operatorname{Cos}\left(\theta_{1}\right)\right] \phi_{1}-\frac{\mathrm{a}}{\mathrm{L}}\left[-\mathrm{K}_{4} \operatorname{Ln}\left(\operatorname{Cos}\left(\theta_{1}\right)\right)-\operatorname{Cos}\left(\theta_{1}\right)^{2}\right]\right\} \\
\mathbf{D}_{12}^{1}= & -\mathrm{K}_{3}\left\{\left[\mathrm{~K}_{4} \operatorname{Ln}\left(\operatorname{Cos}\left(\theta_{2}\right)\right)-\operatorname{Cos}\left(\theta_{2}\right)^{2}\right] \phi_{1}-\frac{\mathrm{a}}{\mathrm{L}}\left[-\mathrm{K}_{4}\left(\operatorname{Tg}\left(\theta_{2}\right)-\theta_{2}\right)-\operatorname{Sen}\left(\theta_{2}\right) \operatorname{Cos}\left(\theta_{2}\right)+\right.\right. \\
& \left.\left.+\theta_{2}\right]\right\}+\mathrm{K}_{3}\left\{\left[\mathrm{~K}_{4} \operatorname{Ln}\left(\operatorname{Cos}\left(\theta_{1}\right)\right)-\operatorname{Cos}\left(\theta_{1}\right)^{2}\right] \phi_{1}-\frac{\mathrm{a}}{\mathrm{L}}\left[-\mathrm{K}_{4}\left(\operatorname{Tg}\left(\theta_{1}\right)-\theta_{1}\right)+\right.\right. \\
& \left.\left.-\operatorname{Sen}\left(\theta_{1}\right) \operatorname{Cos}\left(\theta_{1}\right)+\theta_{1}\right]\right\} \\
\mathrm{D}_{21}^{1}= & -\mathrm{K}_{3}\left\{\left[-\mathrm{K}_{4} \operatorname{Ln}\left(\operatorname{Cos}\left(\theta_{2}\right)\right)-\operatorname{Cos}\left(\theta_{2}\right)^{2}\right] \phi_{1}-\frac{\mathrm{a}}{\mathrm{L}}\left[\mathrm{K}_{4}\left(\operatorname{Tg}\left(\theta_{2}\right)-\theta_{2}\right)-\operatorname{Sen}\left(\theta_{2}\right) \operatorname{Cos}\left(\theta_{2}\right)+\right.\right. \\
& \left.\left.+\theta_{2}\right]\right\}+\mathrm{K}_{3}\left\{\left[-\mathrm{K}_{4} \operatorname{Ln}\left(\operatorname{Cos}\left(\theta_{1}\right)\right)-\operatorname{Cos}\left(\theta_{1}\right)^{2}\right] \phi_{1}-\frac{\mathrm{a}}{\mathrm{L}}\left[\mathrm{K}_{4}\left(\operatorname{Tg}\left(\theta_{1}\right)-\theta_{1}\right)+\right.\right. \\
& \left.\left.-\operatorname{Sen}\left(\theta_{1}\right) \operatorname{Cos}\left(\theta_{1}\right)+\theta_{1}\right]\right\} \\
& +\mathrm{K}_{3}\left\{\left[\left(-\mathrm{K}_{4}+1\right) \theta_{1}-\operatorname{Sen}\left(\theta_{1}\right) \operatorname{Cos}\left(\theta_{1}\right)\right] \phi_{1}-\frac{\mathrm{a}}{\mathrm{L}}\left[\left(\mathrm{K}_{4}-2\right) \operatorname{Ln}\left(\operatorname{Cos}\left(\theta_{1}\right)\right)-\operatorname{Sen}\left(\theta_{1}\right)^{2}\right]\right\} \\
\mathrm{D}_{22}^{1}= & -\mathrm{K}_{3}\left\{\left[\left(\mathrm{~K}_{4}+1\right) \theta_{2}-\operatorname{Sen}\left(\theta_{2}\right) \operatorname{Cos}\left(\theta_{2}\right)\right] \phi_{1}-\frac{\mathrm{a}}{\mathrm{L}}\left[\left(-\mathrm{K}_{4}-2\right) \operatorname{Ln}\left(\operatorname{Cos}\left(\theta_{2}\right)\right)-\operatorname{Sen}\left(\theta_{2}\right)^{2}\right]\right\}+ \\
& +\mathrm{K}_{3}\left\{\left[\left(\mathrm{~K}_{4}+1\right) \theta_{1}-\operatorname{Sen}\left(\theta_{1}\right) \operatorname{Cos}\left(\theta_{1}\right)\right] \phi_{1}-\frac{\mathrm{a}}{\mathrm{L}}\left[\left(-\mathrm{K}_{4}-2\right) \operatorname{Ln}\left(\operatorname{Cos}\left(\theta_{1}\right)\right)-\operatorname{Sen}\left(\theta_{1}\right)^{2}\right]\right\} \\
\mathrm{D}_{31}^{1}= & -\mathrm{K}_{3}\left\{\left[\left(-\mathrm{K}_{4}+1\right) \theta_{2}-\operatorname{Sen}\left(\theta_{2}\right) \operatorname{Cos}\left(\theta_{2}\right)\right] \phi_{1}-\frac{\mathrm{a}}{\mathrm{L}}\left[\left(\mathrm{K}_{4}-2\right) \operatorname{Ln}\left(\operatorname{Cos}\left(\theta_{2}\right)\right)-\operatorname{Sen}\left(\theta_{2}\right)^{2}\right]\right\}+ \\
&
\end{aligned}
$$




$$
\begin{aligned}
D_{32}^{1}= & -K_{3}\left\{\left[\left(-K_{4}-2\right) \operatorname{Ln}\left(\operatorname{Cos}\left(\theta_{2}\right)\right)-\operatorname{Sen}\left(\theta_{2}\right)^{2}\right] \phi_{1}-\frac{a}{L}\left[K_{4}\left(\operatorname{Tg}\left(\theta_{2}\right)-\theta_{2}\right)+\frac{2 \operatorname{Sen}\left(\theta_{2}\right)^{5}}{\operatorname{Cos}\left(\theta_{2}\right)}+\right.\right. \\
& \left.\left.+2 \operatorname{Sen}\left(\theta_{2}\right)^{3}+\operatorname{Cos}\left(\theta_{2}\right)+3 \operatorname{Cos}\left(\theta_{2}\right) \operatorname{Sen}\left(\theta_{2}\right)-3 \theta_{2}\right]\right\}+K_{3}\left\{\left[\left(-K_{4}-2\right) \operatorname{Ln}\left(\operatorname{Cos}\left(\theta_{1}\right)\right)+\right.\right. \\
& \left.-\operatorname{Sen}\left(\theta_{1}\right)^{2}\right] \phi_{1}-\frac{a}{L}\left[K_{4}\left(\operatorname{Tg}\left(\theta_{1}\right)-\theta_{1}\right)+\frac{2 \operatorname{Sen}\left(\theta_{1}\right)^{5}}{\operatorname{Cos}\left(\theta_{1}\right)}+2 \operatorname{Sen}\left(\theta_{1}\right)^{3}+\operatorname{Cos}\left(\theta_{1}\right)+\right. \\
& \left.\left.+3 \operatorname{Cos}\left(\theta_{1}\right) \operatorname{Sen}\left(\theta_{1}\right)-3 \theta_{1}\right]\right\}
\end{aligned}
$$

$$
\begin{aligned}
D_{11}^{2}= & -K_{3}\left\{\left[\left(K_{4}+1\right) \theta_{2}+\operatorname{Sen}\left(\theta_{2}\right) \operatorname{Cos}\left(\theta_{2}\right)\right] \phi_{2}+\frac{a}{L}\left[-K_{4} \operatorname{Ln}\left(\operatorname{Cos}\left(\theta_{2}\right)\right)-\operatorname{Cos}\left(\theta_{2}\right)^{2}\right]\right\}+ \\
& +K_{3}\left\{\left[\left(K_{4}+1\right) \theta_{1}+\operatorname{Sen}\left(\theta_{1}\right) \operatorname{Cos}\left(\theta_{1}\right)\right] \phi_{2}+\frac{a}{L}\left[-K_{4} \operatorname{Ln}\left(\operatorname{Cos}\left(\theta_{1}\right)\right)-\operatorname{Cos}\left(\theta_{1}\right)^{2}\right]\right\} \\
D_{12}^{2}= & -K_{3}\left\{\left[K_{4} \operatorname{Ln}\left(\operatorname{Cos}\left(\theta_{2}\right)\right)-\operatorname{Cos}\left(\theta_{2}\right)^{2}\right] \phi_{2}+\frac{a}{L}\left[-K_{4}\left(\operatorname{Tg}\left(\theta_{2}\right)-\theta_{2}\right)-\operatorname{Sen}\left(\theta_{2}\right) \operatorname{Cos}\left(\theta_{2}\right)+\right.\right. \\
& \left.\left.+\theta_{2}\right]\right\}+K_{3}\left\{\left[K_{4} \operatorname{Ln}\left(\operatorname{Cos}\left(\theta_{1}\right)\right)-\operatorname{Cos}\left(\theta_{1}\right)^{2}\right] \phi_{2}+\frac{a}{L}\left[-K_{4}\left(\operatorname{Tg}\left(\theta_{1}\right)-\theta_{1}\right)+\right.\right. \\
& \left.\left.-\operatorname{Sen}\left(\theta_{1}\right) \operatorname{Cos}\left(\theta_{1}\right)+\theta_{1}\right]\right\}
\end{aligned}
$$

$$
\begin{aligned}
D_{21}^{2}= & -K_{3}\left\{\left[-K_{4} \operatorname{Ln}\left(\operatorname{Cos}\left(\theta_{2}\right)\right)-\operatorname{Cos}\left(\theta_{2}\right)^{2}\right] \phi_{2}+\frac{a}{L}\left[K_{4}\left(\operatorname{Tg}\left(\theta_{2}\right)-\theta_{2}\right)-\operatorname{Sen}\left(\theta_{2}\right) \operatorname{Cos}\left(\theta_{2}\right)+\right.\right. \\
& \left.\left.+\theta_{2}\right]\right\}+K_{3}\left\{\left[-K_{4} \operatorname{Ln}\left(\operatorname{Cos}\left(\theta_{1}\right)\right)-\operatorname{Cos}\left(\theta_{1}\right)^{2}\right] \phi_{2}+\frac{a}{L}\left[K_{4}\left(\operatorname{Tg}\left(\theta_{1}\right)-\theta_{1}\right)+\right.\right. \\
& \left.\left.-\operatorname{Sen}\left(\theta_{1}\right) \operatorname{Cos}\left(\theta_{1}\right)+\theta_{1}\right]\right\}
\end{aligned}
$$

$$
\begin{aligned}
D_{22}^{2}= & -K_{3}\left\{\left[\left(K_{4}+1\right) \theta_{2}-\operatorname{Sen}\left(\theta_{2}\right) \operatorname{Cos}\left(\theta_{2}\right)\right] \phi_{2}+\frac{a}{\mathrm{~L}}\left[\left(-\mathrm{K}_{4}-2\right) \operatorname{Ln}\left(\operatorname{Cos}\left(\theta_{2}\right)\right)-\operatorname{Sen}\left(\theta_{2}\right)^{2}\right]\right\}+ \\
& +\mathrm{K}_{3}\left\{\left[\left(\mathrm{~K}_{4}+1\right) \theta_{1}-\operatorname{Sen}\left(\theta_{1}\right) \operatorname{Cos}\left(\theta_{1}\right)\right] \phi_{2}+\frac{\mathrm{a}}{\mathrm{L}}\left[\left(-\mathrm{K}_{4}-2\right) \operatorname{Ln}\left(\operatorname{Cos}\left(\theta_{1}\right)\right)-\operatorname{Sen}\left(\theta_{1}\right)^{2}\right]\right\} \\
\mathrm{D}_{31}^{2}= & -\mathrm{K}_{3}\left\{\left[\left(-\mathrm{K}_{4}+1\right) \theta_{2}-\operatorname{Sen}\left(\theta_{2}\right) \operatorname{Cos}\left(\theta_{2}\right)\right] \phi_{2}+\frac{\mathrm{a}}{\mathrm{L}}\left[\left(\mathrm{K}_{4}-2\right) \operatorname{Ln}\left(\operatorname{Cos}\left(\theta_{2}\right)\right)-\operatorname{Sen}\left(\theta_{2}\right)^{2}\right]\right\}+ \\
& +\mathrm{K}_{3}\left\{\left[\left(-\mathrm{K}_{4}+1\right) \theta_{1}-\operatorname{Sen}\left(\theta_{1}\right) \operatorname{Cos}\left(\theta_{1}\right)\right] \phi_{2}+\frac{\mathrm{a}}{\mathrm{L}}\left[\left(\mathrm{K}_{4}-2\right) \operatorname{Ln}\left(\operatorname{Cos}\left(\theta_{1}\right)\right)-\operatorname{Sen}\left(\theta_{1}\right)^{2}\right]\right\}
\end{aligned}
$$




$$
\begin{aligned}
D_{32}^{2} & =-K_{3}\left\{\left[\left(-K_{4}-2\right) \operatorname{Ln}\left(\operatorname{Cos}\left(\theta_{2}\right)\right)-\operatorname{Sen}\left(\theta_{2}\right)^{2}\right] \phi_{2}+\frac{a}{L}\left[K_{4}\left(\operatorname{Tg}\left(\theta_{2}\right)-\theta_{2}\right)+\frac{2 \operatorname{Sen}\left(\theta_{2}\right)^{5}}{\operatorname{Cos}\left(\theta_{2}\right)}+\right.\right. \\
& \left.\left.+2 \operatorname{Sen}\left(\theta_{2}\right)^{3}+\operatorname{Cos}\left(\theta_{2}\right)+3 \operatorname{Cos}\left(\theta_{2}\right) \operatorname{Sen}\left(\theta_{2}\right)-3 \theta_{2}\right]\right\}+K_{3}\left\{\left[\left(-K_{4}-2\right) \operatorname{Ln}\left(\operatorname{Cos}\left(\theta_{1}\right)\right)+\right.\right. \\
& \left.-\operatorname{Sen}\left(\theta_{1}\right)^{2}\right] \phi_{2}+\frac{a}{L}\left[K_{4}\left(\operatorname{Tg}\left(\theta_{1}\right)-\theta_{1}\right)+\frac{2 \operatorname{Sen}\left(\theta_{1}\right)^{5}}{\operatorname{Cos}\left(\theta_{1}\right)}+2 \operatorname{Sen}\left(\theta_{1}\right)^{3}+\operatorname{Cos}\left(\theta_{1}\right)+\right. \\
& \left.\left.+3 \operatorname{Cos}\left(\theta_{1}\right) \operatorname{Sen}\left(\theta_{1}\right)-3 \theta_{1}\right]\right\}
\end{aligned}
$$

\section{D.2 Ponto FONTE ALinhado COM O ELEMENTO, POSICIONAdO ATRÁS DO MESMO}

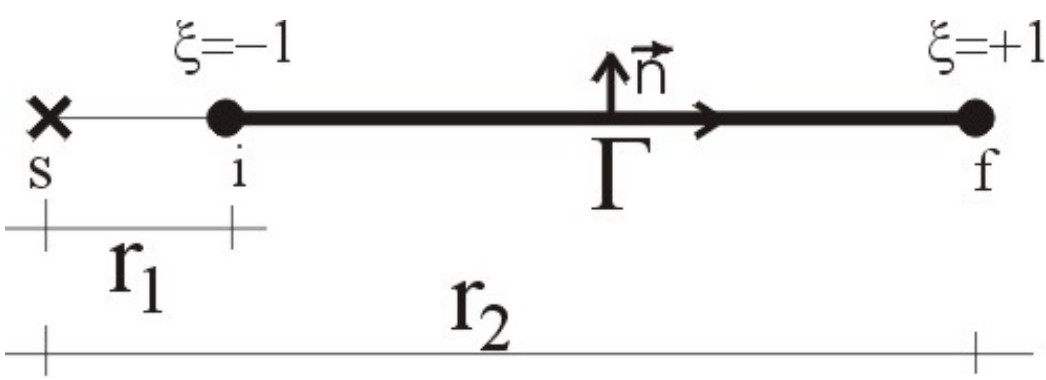

Figura D.2 - Ponto fonte alinhado com o elemento, posicionado atrás do mesmo

\section{Matriz H:}

$\mathrm{H}_{12}^{1}=\mathrm{K}_{3}{ }^{*} \mathrm{~K}_{4}\left[\operatorname{Ln}\left(\mathrm{r}_{2}\right) \phi_{1}-\frac{\mathrm{r}_{2}}{\mathrm{~L}}\right]-\mathrm{K}_{3}{ }^{*} \mathrm{~K}_{4}\left[\operatorname{Ln}\left(\mathrm{r}_{1}\right) \phi_{1}-\frac{\mathrm{r}_{1}}{\mathrm{~L}}\right]$

$\mathrm{H}_{12}^{2}=\mathrm{K}_{3}{ }^{*} \mathrm{~K}_{4}\left[\operatorname{Ln}\left(\mathrm{r}_{2}\right) \phi_{2}+\frac{\mathrm{r}_{2}}{\mathrm{~L}}\right]-\mathrm{K}_{3}{ }^{*} \mathrm{~K}_{4}\left[\operatorname{Ln}\left(\mathrm{r}_{1}\right) \phi_{2}+\frac{\mathrm{r}_{1}}{\mathrm{~L}}\right]$

$\mathrm{H}_{21}^{1}=-\mathrm{H}_{12}^{1}$

$\mathrm{H}_{21}^{2}=-\mathrm{H}_{12}^{2}$

$\mathrm{H}_{11}^{1}=\mathrm{H}_{11}^{2}=\mathrm{H}_{22}^{1}=\mathrm{H}_{22}^{2}=0$

\section{Matriz G:}




$$
\begin{aligned}
& \mathrm{G}_{11}^{1}=\mathrm{K}_{1} *\left\{\mathrm{~K}_{6}\left[\left(\operatorname{Ln}\left(\mathrm{r}_{2}\right) \mathrm{r}_{2}-\mathrm{r}_{2}\right) \phi_{1}-\frac{1}{\mathrm{~L}}\left(\frac{1}{2} \mathrm{r}_{2}^{2} \operatorname{Ln}\left(\mathrm{r}_{2}\right)-\frac{1}{4} \mathrm{r}_{2}^{2}\right)\right]\right\}+ \\
& -\mathrm{K}_{1} *\left\{\mathrm{~K}_{6}\left[\left(\operatorname{Ln}\left(\mathrm{r}_{1}\right) \mathrm{r}_{1}-\mathrm{r}_{1}\right) \phi_{1}-\frac{1}{\mathrm{~L}}\left(\frac{1}{2} \mathrm{r}_{1}^{2} \operatorname{Ln}\left(\mathrm{r}_{1}\right)-\frac{1}{4} \mathrm{r}_{1}^{2}\right)\right]\right\} \\
& \mathrm{G}_{11}^{2}=\mathrm{K}_{1} *\left\{\mathrm{~K}_{6}\left[\left(\operatorname{Ln}\left(\mathrm{r}_{2}\right) \mathrm{r}_{2}-\mathrm{r}_{2}\right) \phi_{2}+\frac{1}{\mathrm{~L}}\left(\frac{1}{2} \mathrm{r}_{2}^{2} \operatorname{Ln}\left(\mathrm{r}_{2}\right)-\frac{1}{4} \mathrm{r}_{2}^{2}\right)\right]\right\}+ \\
& -\mathrm{K}_{1} *\left\{\mathrm{~K}_{6}\left[\left(\operatorname{Ln}\left(\mathrm{r}_{1}\right) \mathrm{r}_{1}-\mathrm{r}_{1}\right) \phi_{2}+\frac{1}{\mathrm{~L}}\left(\frac{1}{2} \mathrm{r}_{1}^{2} \operatorname{Ln}\left(\mathrm{r}_{1}\right)-\frac{1}{4} \mathrm{r}_{1}^{2}\right)\right]\right\} \\
& \mathrm{G}_{22}^{1}=\mathrm{K}_{1} *\left\{\mathrm{~K}_{6}\left[\left(\operatorname{Ln}\left(\mathrm{r}_{2}\right) \mathrm{r}_{2}-\mathrm{r}_{2}\right) \phi_{1}-\frac{1}{\mathrm{~L}}\left(\frac{1}{2} \mathrm{r}_{2}^{2} \operatorname{Ln}\left(\mathrm{r}_{2}\right)-\frac{1}{4} \mathrm{r}_{2}^{2}\right)\right]-\mathrm{r}_{2} \phi_{1}+\frac{\mathrm{r}_{2}^{2}}{2 \mathrm{~L}}\right\}+ \\
& -K_{1} *\left\{K_{6}\left[\left(\operatorname{Ln}\left(r_{1}\right) r_{1}-r_{1}\right) \phi_{1}-\frac{1}{L}\left(\frac{1}{2} r_{1}^{2} \operatorname{Ln}\left(r_{1}\right)-\frac{1}{4} r_{1}^{2}\right)\right]-r_{1} \phi_{1}+\frac{r_{1}^{2}}{2 L}\right\} \\
& \mathrm{G}_{22}^{2}=\mathrm{K}_{1} *\left\{\mathrm{~K}_{6}\left[\left(\operatorname{Ln}\left(\mathrm{r}_{2}\right) \mathrm{r}_{2}-\mathrm{r}_{2}\right) \phi_{2}+\frac{1}{\mathrm{~L}}\left(\frac{1}{2} \mathrm{r}_{2}^{2} \operatorname{Ln}\left(\mathrm{r}_{2}\right)-\frac{1}{4} \mathrm{r}_{2}^{2}\right)\right]-\mathrm{r}_{2} \phi_{2}-\frac{\mathrm{r}_{2}^{2}}{2 \mathrm{~L}}\right\}+ \\
& -\mathrm{K}_{1}^{*}\left\{\mathrm{~K}_{6}\left[\left(\operatorname{Ln}\left(\mathrm{r}_{1}\right) \mathrm{r}_{1}-\mathrm{r}_{1}\right) \phi_{2}+\frac{1}{\mathrm{~L}}\left(\frac{1}{2} \mathrm{r}_{1}^{2} \mathrm{Ln}\left(\mathrm{r}_{1}\right)-\frac{1}{4} \mathrm{r}_{1}^{2}\right)\right]-\mathrm{r}_{1} \phi_{2}-\frac{\mathrm{r}_{1}^{2}}{2 \mathrm{~L}}\right\} \\
& \mathrm{G}_{12}^{1}=\mathrm{G}_{21}^{1}=\mathrm{G}_{12}^{2}=\mathrm{G}_{21}^{2}=0
\end{aligned}
$$

\section{Matriz S}

$$
\begin{aligned}
\mathrm{S}_{11}^{1}= & \mathrm{K}_{7} *\left\{\left[\left(2 \mathrm{~K}_{4}-\mathrm{K}_{5}\right)\left(-\frac{1}{\mathrm{r}_{2}}\right)\right] \phi_{1}-\frac{1}{\mathrm{~L}}\left[\left(2 \mathrm{~K}_{4}-\mathrm{K}_{5}\right) \mathrm{Ln}\left(\mathrm{r}_{2}\right)\right]\right\}+ \\
& -\mathrm{K}_{7}^{*}\left\{\left[\left(2 \mathrm{~K}_{4}-\mathrm{K}_{5}\right)\left(-\frac{1}{\mathrm{r}_{1}}\right)\right] \phi_{1}-\frac{1}{\mathrm{~L}}\left[\left(2 \mathrm{~K}_{4}-\mathrm{K}_{5}\right) \mathrm{Ln}\left(\mathrm{r}_{1}\right)\right]\right\} \\
\mathrm{S}_{11}^{2} & =\mathrm{K}_{7}^{*}\left\{\left[\left(2 \mathrm{~K}_{4}-\mathrm{K}_{5}\right)\left(-\frac{1}{\mathrm{r}_{2}}\right)\right] \phi_{2}+\frac{1}{\mathrm{~L}}\left[\left(2 \mathrm{~K}_{4}-\mathrm{K}_{5}\right) \mathrm{Ln}\left(\mathrm{r}_{2}\right)\right]\right\}+ \\
& -\mathrm{K}_{7}^{*}\left\{\left[\left(2 \mathrm{~K}_{4}-\mathrm{K}_{5}\right)\left(-\frac{1}{\mathrm{r}_{1}}\right)\right] \phi_{2}+\frac{1}{\mathrm{~L}}\left[\left(2 \mathrm{~K}_{4}-\mathrm{K}_{5}\right) \mathrm{Ln}\left(\mathrm{r}_{1}\right)\right]\right\}
\end{aligned}
$$




$$
\begin{aligned}
\mathrm{S}_{22}^{1}= & \mathrm{K}_{7} *\left\{\left[\left(\mathrm{~K}_{4}+2 v\right)\left(-\frac{1}{\mathrm{r}_{2}}\right)\right] \phi_{1}-\frac{1}{\mathrm{~L}}\left[\left(\mathrm{~K}_{4}+2 v\right) \mathrm{Ln}\left(\mathrm{r}_{2}\right)\right]\right\}+ \\
& -\mathrm{K}_{7} *\left\{\left[\left(\mathrm{~K}_{4}+2 v\right)\left(-\frac{1}{\mathrm{r}_{1}}\right)\right] \phi_{1}-\frac{1}{\mathrm{~L}}\left[\left(\mathrm{~K}_{4}+2 v\right) \mathrm{Ln}\left(\mathrm{r}_{1}\right)\right]\right\} \\
\mathrm{S}_{22}^{2} & =\mathrm{K}_{7}^{*}\left\{\left[\left(\mathrm{~K}_{4}+2 v\right)\left(-\frac{1}{\mathrm{r}_{2}}\right)\right] \phi_{2}+\frac{1}{\mathrm{~L}}\left[\left(\mathrm{~K}_{4}+2 v\right) \mathrm{Ln}\left(\mathrm{r}_{2}\right)\right]\right\}+ \\
& -\mathrm{K}_{7}^{*}\left\{\left[\left(\mathrm{~K}_{4}+2 v\right)\left(-\frac{1}{\mathrm{r}_{1}}\right)\right] \phi_{2}+\frac{1}{\mathrm{~L}}\left[\left(\mathrm{~K}_{4}+2 v\right) \mathrm{Ln}\left(\mathrm{r}_{1}\right)\right]\right\} \\
\mathrm{S}_{31}^{1}=\mathrm{S}_{11}^{1} & \\
\mathrm{~S}_{31}^{2}= & \mathrm{S}_{11}^{2} \\
\mathrm{~S}_{12}^{1}= & \mathrm{S}_{21}^{1}=\mathrm{S}_{32}^{1}=\mathrm{S}_{12}^{2}=\mathrm{S}_{21}^{2}=\mathrm{S}_{32}^{2}=0
\end{aligned}
$$

\section{Matriz D}

$$
\begin{aligned}
& D_{12}^{1}=K_{3}{ }^{*} K_{4}\left\{-\left(\frac{r_{2}}{L}\right)-\phi_{2} \operatorname{Ln}\left(r_{2}\right)+\operatorname{Ln}\left(r_{2}\right)+\left(\frac{r_{1}}{L}\right)-\phi_{2} \operatorname{Ln}\left(r_{1}\right)-\operatorname{Ln}\left(r_{1}\right)\right\} \\
& D_{12}^{2}=K_{3} * K_{4}\left\{\left(\frac{r_{2}}{L}\right)+\phi_{2} \operatorname{Ln}\left(r_{2}\right)-\left(\frac{r_{1}}{L}\right)-\phi_{2} \operatorname{Ln}\left(r_{1}\right)\right\} \\
& D_{32}^{1}=\left(-K_{3}{ }^{*} K_{4}-2 K_{3}\right)\left\{-\left(\frac{r_{2}}{L}\right)+\phi_{2} \operatorname{Ln}\left(r_{2}\right)+\operatorname{Ln}\left(r_{2}\right)+\left(\frac{r_{1}}{L}\right)+\phi_{2} \operatorname{Ln}\left(r_{1}\right)-\operatorname{Ln}\left(r_{1}\right)\right\} \\
& D_{12}^{2}=\left(-K_{3}{ }^{*} K_{4}-2 K_{3}\right)\left\{\left(\frac{r_{2}}{L}\right)+\phi_{2} \operatorname{Ln}\left(r_{2}\right)-\left(\frac{r_{1}}{L}\right)-\phi_{2} \operatorname{Ln}\left(r_{1}\right)\right\} \\
& D_{21}^{1}=-D_{12}^{1} \\
& D_{21}^{2}=-D_{12}^{2} \\
& D_{11}^{1}=D_{22}^{1}=D_{31}^{1}=D_{11}^{2}=D_{22}^{2}=D_{31}^{2}=0
\end{aligned}
$$




\section{D.3 Ponto fonte alinhado CoM o elemento, posicionado a fRente do} MESMO

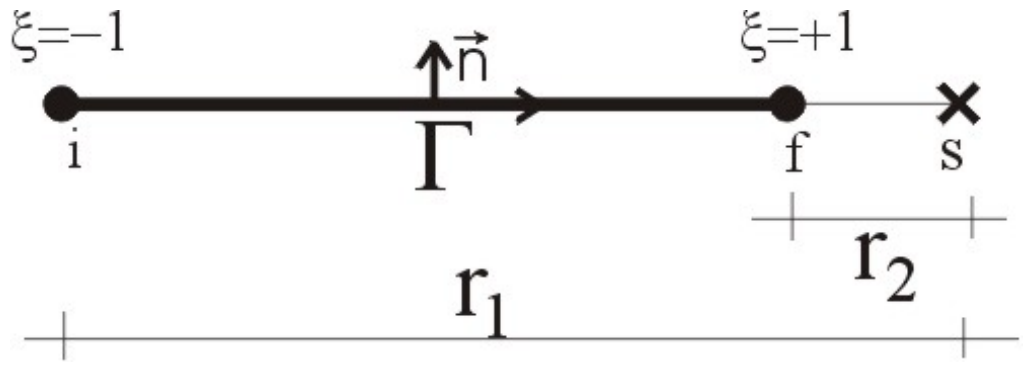

Figura D.3 - Ponto fonte alinhado com o elemento, posicionado a frente do mesmo

\section{Matriz H:}

$\mathrm{H}_{12}^{1}=\mathrm{K}_{3}{ }^{*} \mathrm{~K}_{4}\left[\operatorname{Ln}\left(\mathrm{r}_{2}\right) \phi_{1}+\frac{\mathrm{r}_{2}}{\mathrm{~L}}\right]-\mathrm{K}_{3}{ }^{*} \mathrm{~K}_{4}\left[\operatorname{Ln}\left(\mathrm{r}_{1}\right) \phi_{1}+\frac{\mathrm{r}_{1}}{\mathrm{~L}}\right]$

$\mathrm{H}_{12}^{2}=\mathrm{K}_{3}{ }^{*} \mathrm{~K}_{4}\left[\operatorname{Ln}\left(\mathrm{r}_{2}\right) \phi_{2}-\frac{\mathrm{r}_{2}}{\mathrm{~L}}\right]-\mathrm{K}_{3}{ }^{*} \mathrm{~K}_{4}\left[\operatorname{Ln}\left(\mathrm{r}_{1}\right) \phi_{2}-\frac{\mathrm{r}_{1}}{\mathrm{~L}}\right]$

$\mathrm{H}_{21}^{1}=-\mathrm{H}_{12}^{1}$

$\mathrm{H}_{21}^{2}=-\mathrm{H}_{12}^{2}$

$\mathrm{H}_{11}^{1}=\mathrm{H}_{11}^{2}=\mathrm{H}_{22}^{1}=\mathrm{H}_{22}^{2}=0$

Matriz G:

$$
\begin{aligned}
\mathrm{G}_{11}^{1} & =-\mathrm{K}_{1} *\left\{\mathrm{~K}_{6}\left[\left(\operatorname{Ln}\left(\mathrm{r}_{2}\right) \mathrm{r}_{2}-\mathrm{r}_{2}\right) \phi_{1}+\frac{1}{\mathrm{~L}}\left(\frac{1}{2} \mathrm{r}_{2}^{2} \mathrm{Ln}\left(\mathrm{r}_{2}\right)-\frac{1}{4} \mathrm{r}_{2}^{2}\right)\right]\right\}+ \\
& +\mathrm{K}_{1}^{*}\left\{\mathrm{~K}_{6}\left[\left(\operatorname{Ln}\left(\mathrm{r}_{1}\right) \mathrm{r}_{1}-\mathrm{r}_{1}\right) \phi_{1}+\frac{1}{\mathrm{~L}}\left(\frac{1}{2} \mathrm{r}_{1}^{2} \operatorname{Ln}\left(\mathrm{r}_{1}\right)-\frac{1}{4} \mathrm{r}_{1}^{2}\right)\right]\right\}
\end{aligned}
$$




$$
\begin{aligned}
\mathrm{G}_{11}^{2}= & -\mathrm{K}_{1} *\left\{\mathrm{~K}_{6}\left[\left(\operatorname{Ln}\left(\mathrm{r}_{2}\right) \mathrm{r}_{2}-\mathrm{r}_{2}\right) \phi_{2}-\frac{1}{\mathrm{~L}}\left(\frac{1}{2} \mathrm{r}_{2}^{2} \operatorname{Ln}\left(\mathrm{r}_{2}\right)-\frac{1}{4} \mathrm{r}_{2}^{2}\right)\right]\right\}+ \\
& +\mathrm{K}_{1} *\left\{\mathrm{~K}_{6}\left[\left(\operatorname{Ln}\left(\mathrm{r}_{1}\right) \mathrm{r}_{1}-\mathrm{r}_{1}\right) \phi_{2}-\frac{1}{\mathrm{~L}}\left(\frac{1}{2} \mathrm{r}_{1}^{2} \mathrm{Ln}\left(\mathrm{r}_{1}\right)-\frac{1}{4} \mathrm{r}_{1}^{2}\right)\right]\right\} \\
\mathrm{G}_{22}^{1}= & -\mathrm{K}_{1} *\left\{\mathrm{~K}_{6}\left[\left(\operatorname{Ln}\left(\mathrm{r}_{2}\right) \mathrm{r}_{2}-\mathrm{r}_{2}\right) \phi_{1}+\frac{1}{\mathrm{~L}}\left(\frac{1}{2} \mathrm{r}_{2}^{2} \operatorname{Ln}\left(\mathrm{r}_{2}\right)-\frac{1}{4} \mathrm{r}_{2}^{2}\right)\right]-\mathrm{r}_{2} \phi_{1}-\frac{\mathrm{r}_{2}^{2}}{2 \mathrm{~L}}\right\}+ \\
& +\mathrm{K}_{1} *\left\{\mathrm{~K}_{6}\left[\left(\operatorname{Ln}\left(\mathrm{r}_{1}\right) \mathrm{r}_{1}-\mathrm{r}_{1}\right) \phi_{1}+\frac{1}{\mathrm{~L}}\left(\frac{1}{2} \mathrm{r}_{1}^{2} \operatorname{Ln}\left(\mathrm{r}_{1}\right)-\frac{1}{4} \mathrm{r}_{1}^{2}\right)\right]-\mathrm{r}_{1} \phi_{1}-\frac{\mathrm{r}_{1}^{2}}{2 \mathrm{~L}}\right\} \\
\mathrm{G}_{22}^{2}= & -\mathrm{K}_{1} *\left\{\mathrm{~K}_{6}\left[\left(\operatorname{Ln}\left(\mathrm{r}_{2}\right) \mathrm{r}_{2}-\mathrm{r}_{2}\right) \phi_{2}-\frac{1}{\mathrm{~L}}\left(\frac{1}{2} \mathrm{r}_{2}^{2} \operatorname{Ln}\left(\mathrm{r}_{2}\right)-\frac{1}{4} \mathrm{r}_{2}^{2}\right)\right]-\mathrm{r}_{2} \phi_{2}+\frac{\mathrm{r}_{2}^{2}}{2 \mathrm{~L}}\right\}+ \\
& +\mathrm{K}_{1}^{*}\left\{\mathrm{~K}_{6}\left[\left(\operatorname{Ln}\left(\mathrm{r}_{1}\right) \mathrm{r}_{1}-\mathrm{r}_{1}\right) \phi_{2}-\frac{1}{\mathrm{~L}}\left(\frac{1}{2} \mathrm{r}_{1}^{2} \operatorname{Ln}\left(\mathrm{r}_{1}\right)-\frac{1}{4} \mathrm{r}_{1}^{2}\right)\right]-\mathrm{r}_{1} \phi_{2}+\frac{\mathrm{r}_{1}^{2}}{2 \mathrm{~L}}\right\} \\
\mathrm{G}_{12}^{1}= & \mathrm{G}_{21}^{1}=\mathrm{G}_{12}^{2}=\mathrm{G}_{21}^{2}=0
\end{aligned}
$$

\section{Matriz S}

$$
\begin{aligned}
& \mathrm{S}_{11}^{1}= \mathrm{K}_{7}^{*}\left\{\left[\left(2 \mathrm{~K}_{4}-\mathrm{K}_{5}\right)\left(\frac{1}{\mathrm{r}_{2}}\right)\right] \phi_{1}-\frac{1}{\mathrm{~L}}\left[\left(2 \mathrm{~K}_{4}-\mathrm{K}_{5}\right) \mathrm{Ln}\left(\mathrm{r}_{2}\right)\right]\right\}+ \\
&-\mathrm{K}_{7}^{*}\left\{\left[\left(2 \mathrm{~K}_{4}-\mathrm{K}_{5}\right)\left(\frac{1}{\mathrm{r}_{1}}\right)\right] \phi_{1}-\frac{1}{\mathrm{~L}}\left[\left(2 \mathrm{~K}_{4}-\mathrm{K}_{5}\right) \mathrm{Ln}\left(\mathrm{r}_{1}\right)\right]\right\} \\
& \mathrm{S}_{11}^{2}=\mathrm{K}_{7}^{*}\left\{\left[\left(2 \mathrm{~K}_{4}-\mathrm{K}_{5}\right)\left(\frac{1}{\mathrm{r}_{2}}\right)\right] \phi_{2}+\frac{1}{\mathrm{~L}}\left[\left(2 \mathrm{~K}_{4}-\mathrm{K}_{5}\right) \mathrm{Ln}\left(\mathrm{r}_{2}\right)\right]\right\}+ \\
&-\mathrm{K}_{7}^{*}\left\{\left[\left(2 \mathrm{~K}_{4}-\mathrm{K}_{5}\right)\left(\frac{1}{\mathrm{r}_{1}}\right)\right] \phi_{2}+\frac{1}{\mathrm{~L}}\left[\left(2 \mathrm{~K}_{4}-\mathrm{K}_{5}\right) \operatorname{Ln}\left(\mathrm{r}_{1}\right)\right]\right\} \\
& \mathrm{S}_{22}^{1}=\mathrm{K}_{7}^{*}\left\{\left[\left(\mathrm{~K}_{4}+2 v\right)\left(\frac{1}{\mathrm{r}_{2}}\right)\right] \phi_{1}-\frac{1}{\mathrm{~L}}\left[\left(\mathrm{~K}_{4}+2 v\right) \operatorname{Ln}\left(\mathrm{r}_{2}\right)\right]\right\}+ \\
&-\mathrm{K}_{7}^{*}\left\{\left[\left(\mathrm{~K}_{4}+2 v\right)\left(\frac{1}{\mathrm{r}_{1}}\right)\right] \phi_{1}-\frac{1}{\mathrm{~L}}\left[\left(\mathrm{~K}_{4}+2 v\right) \mathrm{Ln}\left(\mathrm{r}_{1}\right)\right]\right\}
\end{aligned}
$$




$$
\begin{aligned}
\mathrm{S}_{22}^{2} & =\mathrm{K}_{7} *\left\{\left[\left(\mathrm{~K}_{4}+2 v\right)\left(\frac{1}{\mathrm{r}_{2}}\right)\right] \phi_{2}+\frac{1}{\mathrm{~L}}\left[\left(\mathrm{~K}_{4}+2 v\right) \mathrm{Ln}\left(\mathrm{r}_{2}\right)\right]\right\}+ \\
& -\mathrm{K}_{7} *\left\{\left[\left(\mathrm{~K}_{4}+2 v\right)\left(\frac{1}{\mathrm{r}_{1}}\right)\right] \phi_{2}+\frac{1}{\mathrm{~L}}\left[\left(\mathrm{~K}_{4}+2 v\right) \mathrm{Ln}\left(\mathrm{r}_{1}\right)\right]\right\} \\
\mathrm{S}_{31}^{1} & =\mathrm{S}_{11}^{1} \\
\mathrm{~S}_{31}^{2} & =\mathrm{S}_{11}^{2} \\
\mathrm{~S}_{12}^{1} & =\mathrm{S}_{21}^{1}=\mathrm{S}_{32}^{1}=\mathrm{S}_{12}^{2}=\mathrm{S}_{21}^{2}=\mathrm{S}_{32}^{2}=0
\end{aligned}
$$

\section{Matriz D}

$$
\begin{aligned}
& D_{12}^{1}=K_{3}{ }^{*} K_{4}\left\{\frac{r_{2}}{L}-\phi_{2} \operatorname{Ln}\left(r_{2}\right)+\operatorname{Ln}\left(r_{2}\right)+\frac{r_{1}}{L}+\phi_{2} \operatorname{Ln}\left(r_{1}\right)-\operatorname{Ln}\left(r_{1}\right)\right\} \\
& D_{12}^{2}=K_{3}{ }^{*} K_{4}\left\{-\frac{r_{2}}{L}+\phi_{2} \operatorname{Ln}\left(r_{2}\right)+\frac{r_{1}}{L}-\phi_{2} \operatorname{Ln}\left(r_{1}\right)\right\} \\
& D_{32}^{1}=\left(-K_{3}{ }^{*} K_{4}-2 K_{3}\right)\left\{\frac{r_{2}}{L}-\phi_{2} \operatorname{Ln}\left(r_{2}\right)+\operatorname{Ln}\left(r_{2}\right)-\frac{r_{1}}{L}-\phi_{2} \operatorname{Ln}\left(r_{1}\right)-\operatorname{Ln}\left(r_{1}\right)\right\} \\
& D_{12}^{2}=\left(-K_{3}{ }^{*} K_{4}-2 K_{3}\right)\left\{\frac{r_{2}}{L}+\phi_{2} \operatorname{Ln}\left(r_{2}\right)+\frac{r_{1}}{L}-\phi_{2} \operatorname{Ln}\left(r_{1}\right)\right\} \\
& D_{21}^{1}=-D_{12}^{1} \\
& D_{21}^{2}=-D_{12}^{2} \\
& D_{11}^{1}=D_{22}^{1}=D_{31}^{1}=D_{11}^{2}=D_{22}^{2}=D_{31}^{2}=0
\end{aligned}
$$




\section{INTEGRAIS ANALÍticas SingULARES}

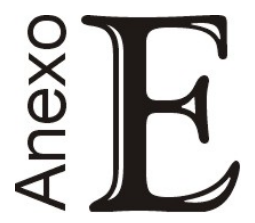

Para facilitar a dedução e implementação, foram empregadas algumas constantes que estão descritas a seguir:

$$
\begin{aligned}
& \mathrm{K}_{1}=\frac{(1-2 v)}{4 \pi(1-v)} \\
& \mathrm{K}_{2}=\frac{\mathrm{L}}{16 \pi \mathrm{G}(1-v)}
\end{aligned}
$$

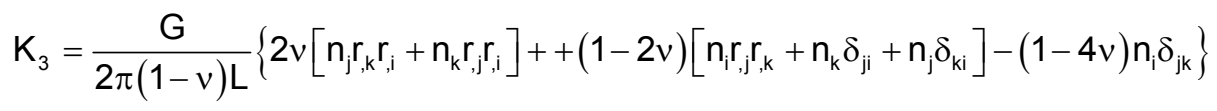

$$
\begin{aligned}
& \mathrm{K}_{4}=\frac{1}{4 \pi(1-v) \mathrm{L}}\left\{-(1-2 \mathrm{v})\left[\mathrm{r}_{, \mathrm{k}} \delta_{i j}+\mathrm{r}_{, j} \delta_{\mathrm{ik}}-\mathrm{r}_{, \mathrm{i}} \delta_{\mathrm{jk}}\right]+2 \mathrm{r}_{\mathrm{i}, \mathrm{r}, \mathrm{r}, \mathrm{k}, \mathrm{k}}\right\}
\end{aligned}
$$

As variáveis $\theta_{1}$ e $\theta_{2}$ são os co-senos diretores da normal ao elemento a ser integrado. 


\section{E.1 Ponto FONTE COINCIDINDo COM O PRIMEIRO NÓ DO ELEMENTO DE INTEGRAÇÃO}

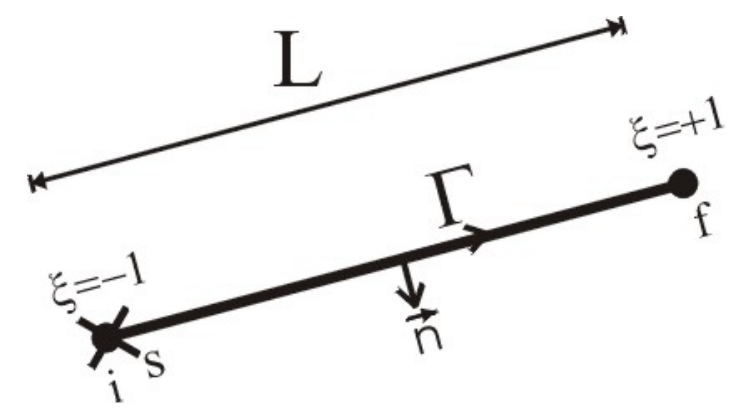

Figura E.1: Ponto fonte coincidindo com o primeiro nó do elemento a ser integrado.

\section{Matriz H:}

$\mathrm{H}_{12}^{1}=\mathrm{K}_{1}(1-\operatorname{Ln}(\mathrm{L}))$

$\mathrm{H}_{12}^{2}=-\mathrm{K}_{1}$

$\mathrm{H}_{21}^{1}=-\mathrm{H}_{12}^{1}$

$\mathrm{H}_{21}^{2}=-\mathrm{H}_{12}^{2}$

$\mathrm{H}_{11}^{1}=\mathrm{H}_{22}^{1}=\mathrm{H}_{11}^{2}=\mathrm{H}_{22}^{2}=0$

\section{Matriz G}

$$
\begin{aligned}
& \mathrm{G}_{11}^{1}=\mathrm{K}_{2}\left[(3-4 v)\left(\frac{3}{2}-\mathrm{Ln}(\mathrm{L})\right)+\theta_{2}^{2}\right] \\
& \mathrm{G}_{22}^{1}=\mathrm{K}_{2}\left[(3-4 v)\left(\frac{3}{2}-\mathrm{Ln}(\mathrm{L})\right)+\theta_{1}^{2}\right] \\
& \mathrm{G}_{11}^{2}=\mathrm{K}_{2}\left[(3-4 v)\left(\frac{1}{2}-\mathrm{Ln}(\mathrm{L})\right)+\theta_{2}^{2}\right]
\end{aligned}
$$




$$
\begin{aligned}
& \mathrm{G}_{22}^{2}=\mathrm{K}_{2}\left[(3-4 v)\left(\frac{1}{2}-\operatorname{Ln}(\mathrm{L})\right)+\theta_{1}^{2}\right] \\
& \mathrm{G}_{12}^{1}=\mathrm{G}_{12}^{2}=\mathrm{G}_{21}^{1}=\mathrm{G}_{21}^{2}=-\mathrm{K}_{2} \theta_{1} \theta_{2}
\end{aligned}
$$

\section{E.2 PONTO FONTE INTERNO AO ELEMENTO DE INTEGRAÇÃo}

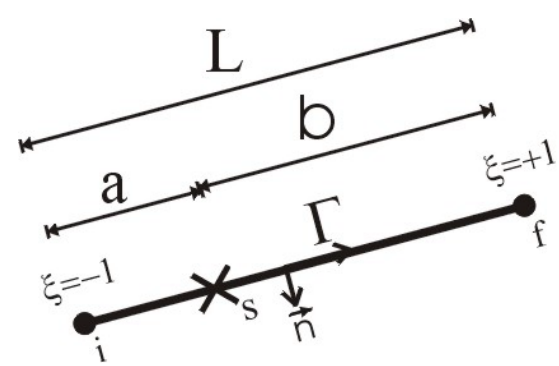

Figura E.2: Ponto fonte interno ao elemento de integração.

\section{Matriz H:}

$H_{12}^{1}=\frac{K_{1}}{L}\left(b L n\left(\frac{b}{a}\right)+L\right)$

$H_{12}^{2}=\frac{K_{1}}{L}\left(a L n\left(\frac{a}{b}\right)+L\right)$

$\mathrm{H}_{21}^{1}=-\mathrm{H}_{12}^{1}$

$\mathrm{H}_{21}^{2}=-\mathrm{H}_{12}^{2}$

$\mathrm{H}_{11}^{1}=\mathrm{H}_{22}^{1}=\mathrm{H}_{11}^{2}=\mathrm{H}_{22}^{2}=0$

\section{Matriz G}

$\mathrm{G}_{11}^{1}=\frac{2 \mathrm{~K}_{2}}{\mathrm{~L}^{2}}\left\{-(3-4 v)\left[\left(\mathrm{ab}+\frac{\mathrm{a}^{2}}{2}\right) \operatorname{Ln}(\mathrm{a})+\frac{\mathrm{b}^{2}}{2} \operatorname{Ln}(\mathrm{b})-\left(\frac{\mathrm{a}^{2}}{4}+\mathrm{ab}+\frac{3 \mathrm{~b}^{2}}{4}\right)+\frac{\mathrm{L}^{2}}{2} \theta_{2}^{2}\right]\right\}$ 


$$
\begin{aligned}
& \mathrm{G}_{22}^{1}=\frac{2 \mathrm{~K}_{2}}{\mathrm{~L}^{2}}\left\{-(3-4 v)\left[\left(a b+\frac{a^{2}}{2}\right) \operatorname{Ln}(a)+\frac{b^{2}}{2} \operatorname{Ln}(b)-\left(\frac{a^{2}}{4}+a b+\frac{3 b^{2}}{4}\right)+\frac{L^{2}}{2} \theta_{1}^{2}\right]\right\} \\
& G_{11}^{2}=\frac{2 K_{2}}{L^{2}}\left\{-(3-4 v)\left[\left(a b+\frac{b^{2}}{2}\right) \operatorname{Ln}(b)+\frac{a^{2}}{2} \operatorname{Ln}(a)-\left(\frac{b^{2}}{4}+a b+\frac{3 a^{2}}{4}\right)+\frac{L^{2}}{2} \theta_{2}^{2}\right]\right\} \\
& G_{22}^{2}=\frac{2 K_{2}}{L^{2}}\left\{-(3-4 v)\left[\left(a b+\frac{b^{2}}{2}\right) \operatorname{Ln}(b)+\frac{a^{2}}{2} \operatorname{Ln}(a)-\left(\frac{b^{2}}{4}+a b+\frac{3 a^{2}}{4}\right)+\frac{L^{2}}{2} \theta_{1}^{2}\right]\right\} \\
& G_{12}^{1}=G_{12}^{2}=G_{21}^{1}=G_{21}^{2}=-K_{2} \theta_{1} \theta_{2}
\end{aligned}
$$

\section{Matriz S}

$$
\begin{aligned}
& S_{i j}^{1}=K_{3}\left\{\operatorname{Ln}(a)-\operatorname{Ln}(b)-\frac{b}{a}-1\right\} \\
& S_{i j}^{2}=K_{3}\left\{-\operatorname{Ln}(a)+\operatorname{Ln}(b)-\frac{a}{b}-1\right\}
\end{aligned}
$$

\section{Matriz D}

$D_{i j}^{1}=K_{4}\{b[\operatorname{Ln}(a)-\operatorname{Ln}(b)+1]+a\}$

$D_{i j}^{2}=K_{4}\{a[\operatorname{Ln}(a)-\operatorname{Ln}(b)-1]-b\}$

\section{E.3 Ponto Fonte COINCIDINDo COM O SEgundo NÓ DO ElEMENTo DE}

\section{INTEGRAÇÃO}

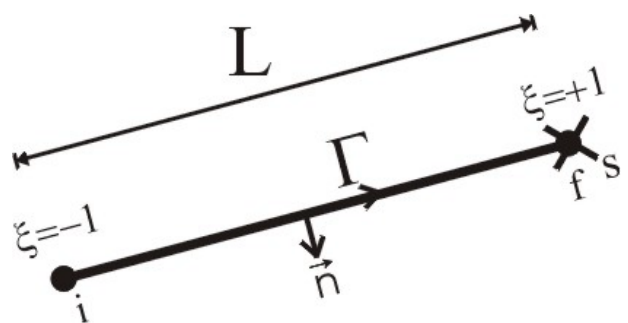

Figura E.3: Ponto fonte coincidindo com o segundo nó do elemento a ser integrado. 


\section{Matriz H:}

$\mathrm{H}_{12}^{1}=\mathrm{K}_{1}$

$\mathrm{H}_{12}^{2}=-\mathrm{K}_{1}(1-\mathrm{Ln}(\mathrm{L}))$

$\mathrm{H}_{21}^{1}=-\mathrm{H}_{12}^{1}$

$\mathrm{H}_{21}^{2}=-\mathrm{H}_{12}^{2}$

$\mathrm{H}_{11}^{1}=\mathrm{H}_{22}^{1}=\mathrm{H}_{11}^{2}=\mathrm{H}_{22}^{2}=0$

\section{Matriz G}

$$
\begin{aligned}
& \mathrm{G}_{11}^{1}=\mathrm{K}_{2}\left[(3-4 v)\left(\frac{1}{2}-\mathrm{Ln}(\mathrm{L})\right)+\theta_{2}^{2}\right] \\
& \mathrm{G}_{22}^{1}=\mathrm{K}_{2}\left[(3-4 v)\left(\frac{1}{2}-\mathrm{Ln}(\mathrm{L})\right)+\theta_{1}^{2}\right] \\
& \mathrm{G}_{11}^{2}=\mathrm{K}_{2}\left[(3-4 v)\left(\frac{3}{2}-\mathrm{Ln}(\mathrm{L})\right)+\theta_{2}^{2}\right] \\
& \mathrm{G}_{22}^{2}=\mathrm{K}_{2}\left[(3-4 v)\left(\frac{3}{2}-\operatorname{Ln}(\mathrm{L})\right)+\theta_{1}^{2}\right] \\
& \mathrm{G}_{12}^{1}=\mathrm{G}_{12}^{2}=\mathrm{G}_{21}^{1}=\mathrm{G}_{21}^{2}=-\mathrm{K}_{2} \theta_{1} \theta_{2}
\end{aligned}
$$




\section{REFERÊNCIAS BIBLIOGRÁFICAS}

AGOSTINHO, P.C.P. (1998). Associação de chapas através da combinação dos métodos dos elementos de contorno e finitos considerando enrijecedores e crack coesivo. São Carlos. Tese (Doutorado) - Escola de Engenharia de São Carlos, Universidade de São Paulo.

ARISTIDERMO, M.; TURCO, E. (1994). Boundary element discretization of plane elasticity and plate bending problems. Int. J. Num. Meth. Engng., Vol.37, 965-987.

BEER, G. (2001). Programming the boundary element method - An introduction for engineers. Chichester. WILEY.

BREBBIA, C.A.; DOMINGUEZ, J. (1984). Boundary element: an introductory course. Southampton, CML Publ.

BREBBIA, C.A.; TELES, J.C.F.; WROBEL, L.C. (1984). Boundary element techniques: Theory and applications in engineering. Berlin. Spinger-Verlag.

BOTTA, A. S.; VENTURINI, W. S. (2003). Reinforced 2D domain analysis using BEM and regularized BEM/FEM combination. (a ser submetido)

CODA, H.B. (1993). Análise tridimensional transiente de estruturas pela combinação entre o método dos elementos de contorno e o método dos elementos finitos. São Carlos. Tese (Doutorado) - Escola de Engenharia de São Carlos, Universidade de São Paulo. 
CODA, H.B. (2001). Dynamic and static non-linear analysis of reinforced media: a BEM/FEM coupling approach. Computers \& Structures, v.79, pp. 2751-2765.

CRUSE, T.A. (1969). Numerical solutions in three dimensional elastostatics. Int. Journal of Solid and Structures, v.5, pp. 1259-1274.

CRUSE, T.A. (1974). An improved boundary-integral equation method for three dimensional elastic stress analysis. Computer and Structures, v. 4, pp. 741-754.

FERRO, N.C.P.; VENTURINI, W.S. (1991). Formulação de um elemento de fundação através do método dos elementos de contorno. In: JORNADAS SUL-AMERICANAS DE ENGENHARIA ESTRUTURAL, 25., Porto Alegre. Anais. V.1, p.421-430.

FERRO, N.C.P.; VENTURINI, W.S. (1992). BEM-FEM coupling for building structure analysis. In: INTERNATIONAL CONFERENCE ON METHODS, 14th., Seville, November. Proceedings

FERRO, N.C.P. (1993). Uma conbinação MEC/MEF para análise de fundação enrijecidas por estacas. São Carlos. Dissertação (mestrado) - Escola de Engenharia de São Carlos, Universidade de São Paulo.

FOLTRAN, C.E. (1999). Análise de problemas planos em regime elasto-plástico pelo método dos elementos de contorno. Campinas. Dissertação (mestrado) - Faculdade de Engenharia Civil, Universidade Estadual de Campinas.

GIL RODRÍGUEZ, J.C. (1986). Sobre o emprego do método dos elementos de contorno em problemas elásticos bidimensionais. São Carlos. Dissertação (Mestrado) - Escola de Engenharia de São Carlos, Universidade de São Paulo.

KOMATSU, J.S. (1995). Estudo de problemas de escavação através da combinação elementos de contorno e elementos finitos. São Carlos. Dissertação (Mestrado) - Escola de Engenharia de São Carlos, Universidade de São Paulo. 
LEITE, L.G.S.; CODA, H.B.; VENTURINI, W.S. (2003). Two-dimensional solids reinforced by thin bars using the boundary element method. Engineering analysis with boundary elements, v. 27, pp. 193-201.

LOPES, M.C.JR. (1996). Modelagem numérica do crescimento de fraturas através do método dos elementos de contorno. São Carlos. Dissertação (Mestrado) - Escola de Engenharia de São Carlos. Universidade de São Paulo.

LOVÓN, O. (2003). Formulação adaptativa do MEC para problemas elásticos. São Carlos. Tese (Doutorado) - Escola de Engenharia de São Carlos, Universidade de São Paulo. (em desenvolvimento)

MACIEL, D.N. (2003). Determinação dos fatores de intensidade de tensão estáticos e dinâmicos via MEC com integração analítica em coordenadas locais. São Carlos. Dissertação (Mestrado) - Escola de Engenharia de São Carlos, Universidade de São Paulo.

MENDONÇA, A.V. (2002). Estudo de estruturas compostas por lâminas planas de espessuras constantes: Uma abordagem pelo método dos elementos de contorno. São Carlos. Tese (Doutorado) - Escola de Engenharia de São Carlos, Universidade de São Paulo.

PAIVA, J.B. (1987). Formulação do método dos elementos de contorno para flexão de placas e suas aplicações em engenharia de estruturas. São Carlos. Tese (Doutorado) Escola de Engenharia de São Carlos, Universidade de São Paulo.

PAIVA, J.B. (1991). Boundary element formulation for plate analysis with special distribution of reactions along the boundary. Advances in Engineering Software and Workstations 13, pp. 162-168, July.

PAIVA, J.B.; ALIVEIRA NETO, L. (1995). Na alternative boundary element formulation for plate bending analysis. In: BETECH 95, Adelaide, Australia. 
PALERMO, L.JR. (1989). Análise de peças delgadas como associação de placas pelo método dos elementos de contorno. São Carlos. Tese (Doutorado) - Escola de Engenharia de São Carlos, Universidade de São Paulo.

PALERMO, L.JR.; RACHID, M.; VENTURINI, W.S. (1992). Analysis of thin walled structures using the boundary element method. Eng. Anal. Bound. Elem., 9, pp. 359-363.

RAMALHO, M.A. (1990). Sistema para análise de estruturas considerando interação com o meio elástico. São Carlos. Tese (Doutorado) - Escola de Engenharia de São Carlos, Universidade de São Paulo.

RAMALHO, M.A. ; VENTURINI, W.S. (1990). Formulação de um elemento para a discretização de sapatas rígidas com base no método dos elementos de contorno. In: CONGRESSO IBERO LATINO AMERICANO SOBRE MÉTODOS COMPUTACIONAIS PARA ENGENHARIA, 11., Rio de Janeiro. Anais. V.2, p.903912.

ROCHA, F.S. (1988). Análise de Descontinuidade pelo Método dos Elementos de Contorno, São Carlos, Escola de Engenharia de São Carlos USP, (tese de doutoramento) (orientador Wilson S. Venturini).

SOUZA, J.L. (1999). A h-adaptabilidade no método dos elementos de contorno (MEC). Algumas considerações sobre singularidade, hipersingularidade e hierarquia. São Carlos. Tese (Doutorado) - Escola de Engenharia de São Carlos, Universidade de São Paulo.

TELLES, J.C.F. (1987). A self-adaptative coordinate transformation for efficient numerical evaluations of general boundary element integrals. Int. J. Num. Meth. Engng., Vol. 24, pp. $959-973$ 
VENTURINI, W.S. (1988). Um estudo sobre o método dos elementos de contorno e suas aplicações em problemas de engenharia. São Carlos. Tese (livre docência). Escola de Engenharia de São Carlos - USP.

\section{Bibliografia COMPlementaR}

ABEL, N.H.(1881). Ouvres complètes Norvegien Christiania, v. 1.

AYALA, F.; GOMEZ, R. (1979). A general procedure for solving three dimensional elasticity problems in geomechanics. In: INT. CONFERENCE ON NUMERICAL METHODS INGEOMECHANICS, 3rd, Aachen, 2-6 April. Proceedings. P.3-13.

BETTY, E. (1872). Teoria dell elasticita. I1 Nuovo Ciemento, v.7-10.

BREBBIA, C.A. (1978a) The Boundary element method for engineers. London, Pentech Press.

BREBBIA, C.A. (1978b) Weighted residual classification of aproximate methods. Appl. Math. Modelling, v.2, n.3.

BREBBIA, C.A.; FERRANTE, A.J. (1978). Computational methods for the solution of engineering problems. London, Pentech Press.

BREBBIA, C.A.; GEORGIO, P. (1980). Combination of boundary and finite elements for elastostatics. Appl. Math. Modelling, v.3, p.212-220.

BREBBIA, C.A.; WALKER, S. (1980). Boundary element techniques in engineering. London, Newnes-Butterworths.

BREBBIA, C.A. (1981). Bondary element methods. Springer-Verlag. 
BREBBIA, C.A. (1982). Bondary element methods in engineering. Springer-Verlag.

BREBBIA, C.A. (1983). Bondary element. Springer-Verlag.

BREBBIA, C.A. (1984). Bondary element techniques: theory and applications in engineering. Springer-Verlag.

CHEN, W.F.; MEI, C.C. (1974). Oscillations and wave forces in a man-made harbour. In: NAVAL HYDRO SYMP., 10th, Dept. of Civil Eng., MIT., Cambridge, USA.

CORNELL, G.; Delphi: Segredos e soluções. Makron Books. São Paulo. 1996. 367p.

CRUSE, T.A. (1973). Application of the boundary-integral equation method to three dimensional stress analysis. Computer and Structures, v. 3, pp. 509-527.

CRUSE, T.A.; VANBUREN, W (1971). Three dimensional elastic stress analysis of a fracture specimem with na edge crack. Int. Journal Mech., v. 7, pp. 1-15.

FREDHOLM, I. (1903). Sur une classe d'equation fonctionelles, Acta Math., v. 27, pp. 365390.

HILL, Francis S. Computer Graphics Using OpenGL. 2nd ed. Upper Saddle River, New Jersey: Prentice-Hall, Inc., 2000. 922 p.

JASWON, M.A. (1963). Integral equation methods in potential theory I. Proc. Royal Society Ser., v. A275, pp. 23-32.

KUPRADZE, V.D. (1965). Potencial methods in the theory of elasticity. Jerusalem: Israel Program for Scientific Translations.

LACHAT, J.C. (1975). A further development of the boundary integral technique for elastostatics. Southampton, Ph. D. Thesis - University of Southampton. 
LIOUVILLE, V. (1837). Sur de développment des functions. Journal de Mathémetiques Pures et Appliqueés, v. 2, pp. 16-35

LONGO, M.B.; Delphi6. Brasport. Rio de Janeiro. 2001

MASSONET, C.E.; ZIENKIEWICZ, O.C., and HOLLISTER, G.S. (1965). Numerical use for integral procedures. Stress analysis. London: Wiley, cap. 10.

McDONALD, B.H.; WEXLER, A. (1972). Finite element solution of unbounded field problems. IEE Trans. Microwave Theory and Techniques, MTT-20, p.841-47.

MIKHLIN, S.G. (1957). Integral equations. London: Pergamon Press.

MIKHLIN, S.G. (1965). Multi-dimensional singulaar integrals and integral equations. Oxford: Pergamon Press.

MIKHLIN, S.G. (1965). Approximate methods for solution of differential and integral equations. Oxford: Pergamon Press.

MUSKHELISHVILI, N.I. (1953). Some basic problems of mathematical theory of elasticity. Groningen, Holand: Noordhoff.

MUSTUE, G.G.; VOLAIT, F. (1980). A symmetric direct integral equation method for twodimensional elastostatics. In: INT. SEMINAR ON BOUNDARY ELEMENT METHODS, 2nd, Southampton. Proceedings.

OSIAS, J.R., et al (1977). Combined boundary integral equation finite element analysis of solids. In: SYMPOSIUM ON INNOVATIVE NUMERICAL ANALYSIS IN APPLIED ENGINEERING SCIENCE, 1st, Versailles, CETIM - Proc.

RIZZO, F.J. (1967). An Integral equation approach to boundary value problems of classic elastostatic. Quarterly of Applied Mathematics, v. 25 n. 1, pp. 83-95. 
SHAW, R.P.; FALBY, W. (1977). FEBIE: A combined finite element-boundary integral equations method. In: SYMPOSIUM ON INNOVATIVE NUMERICAL ANALYSIS IN APLLIED ENGINEERING SCIENCE, 1st, Versailles. Proceedings.

SOMIGLIANA, C. (1886). Sopra l'equilibrio di un corpo elastico isotropo. I1 Nuovo Ciemento, V. 17-19.

SYMM, G.T. (1963). Integral equation methods in potential theory II. Proc. Royal Society Ser., v. A275, pp. 33-46.

TELLES, J.C.F.; BREBBIA, C.A. (1979). On the aplication of the boundary element methodo to plasticity. Appl. Math. Modelling, v.3, p.466-470.

TELLES, J.C.F.; BREBBIA, C.A. (1980a). The boundary element method in plasticity. In: BREBBIA, C.A., ED. New development in boundary element methods. CML Publications, p.295-317.

TELLES, J.C.F.; BREBBIA, C.A. (1980b). Elastoplastic boundary element analysis. In: WUNDERLICH, W. et alii, eds. Proc. Europe-U.S. Workshop on nonlinear finite element analysis in structural mechanics. Springer-Verlag, p. 403-434.

TIMOSHENKO, S.P.; GOODIER, J.N. (1980). Teoria da Elasticidade, Guanabara Dois, Rio de Janeiro, Brasil.

VENTURINI, W.S. (1982). Application of the boundary element formulation to solve geomechanical problems. Southampton, Ph. D. Thesis - University of Southampton.

VENTURINI, W.S. ; BREBBIA, C.A. (1983). Some applications of the boundary element method in geomechanics. Int. J. Num. Anal. Meth. Geomech., v.7, p.419-434.

VENTURINI, W.S. (1984). Boundary element method in geomechanics. Springer-Verlag. (Lecture Notes in Engineering). 
VENTURINI, W.S. ; BREBBIA, C.A. (1988). Boundary element formulation for nonlinear applications in geomechanics. Appl. Math. Modelling, V.8, pp. 251-260.

VOLTERRA, V. (1956). Opere mathematiche. Acad. Naz. Lincei, Rome, v.2, p216-275.

WOO, Mason; NEIDER, Jackie; DAVIS, Tom; SHREINER, Dave. OpenGL Programming Guide: the official guide to learning OpenGL, version 1.2. 3rd ed. Reading, Massachusetts: Addison Wesley, 1999. 730 p.

WRIGHT, Richard S. Jr.; SWEET, Michael. OpenGL SuperBible. 2nd ed. Indianapolis, Indiana: Waite Group Press, 2000. 696 p.

ZIENKIEWICZ, O.C., et al. (1977). The coupling of the finite element method and boundary solution procedures. Int. J. Num. Meth. Engng., v.11, p.355-375. 
A notação indicial é uma forma compacta de se representar e manipular sistemas de equações, combinações lineares e somatórios. Em várias situações, a notação indicial é bastante útil, como por exemplo, aos se tratar como equações constitutivas de materiais.

Basicamente, deve-se definir o conceito de notação indicial, o significado de índices repetidos e livres e as operações empregando estes índices.

\section{a.1) Definição}

Um conjunto de variáveis $\mathrm{x}_{1}, \mathrm{x}_{2}, \ldots, \mathrm{x}_{\mathrm{n}}$ é geralmente denotado como $\mathrm{x}_{\mathrm{i}}(\mathrm{i}=1,2, \ldots$, n). Quando escrito isoladamente, o símbolo $x_{i}$ indica qualquer uma das variáveis $x_{1}, x_{2}, \ldots$, $\mathrm{x}_{\mathrm{n}}$. $\mathrm{O}$ intervalo de variação do índice $\mathrm{i}(\mathrm{i}=1,2, \ldots, \mathrm{n})$ deve ser sempre dado. Este índice pode ser denotado como um subscrito ou sobrescrito, ou seja, $x_{i}$ ou $x^{i}$ são ambos válidos. Um sistema de notações usando índices é denominado notação indicial.

\section{a.2) Somatório}

Considere a equação de um plano no sistema de referência cartesiano tridimensional com eixos $\mathrm{x}_{1}, \mathrm{x}_{2}, \mathrm{x}_{3}$ 
$a_{1} x_{1}+a_{2} x_{2}+a_{3} x_{3}=p$

Sendo $\mathrm{a}_{1}, \mathrm{a}_{2}, \mathrm{a}_{3}$ e p constantes. Usualmente a expressão anterior é escrita como

$\mathrm{ax}+\mathrm{by}+\mathrm{cz}=\mathrm{d}$

A notação indicial permite escrever as expressões numa forma compacta, denotamse as expressões como em (A.1). Essa equação pode ser escrita como em termos do seguinte somatório

$\sum_{\mathrm{i}=1}^{3} \mathrm{a}_{\mathrm{i}} \mathrm{x}_{\mathrm{i}}=\mathrm{p}$.

Introduzindo a notação de somatório denota-se a equação anterior como

$\mathrm{a}_{\mathrm{i}} \mathrm{x}_{\mathrm{i}}=\mathrm{p}$.

A convenção é a seguinte: a repetição de um índice num termo representará um somatório com respeito a esse índice no seu intervalo de variação. $\mathrm{O}$ intervalo de variação de um índice é o conjunto de números inteiros de $1 \mathrm{a}$ n. Como este índice é empregado apenas para uma soma é chamado índice falso ou repetido, pois o símbolo usado no somatório se torna indiferente no resultado final. Assim, por exemplo, $\mathrm{a}_{\mathrm{i}} \mathrm{x}_{\mathrm{i}}$ pode ser denotado como $\mathrm{a}_{\mathrm{j}} \mathrm{x}_{\mathrm{j}}$ sem alterar o significado da expressão. Um índice que não é somado é denominado índice livre e indica o número de equações associado ao termo em notação indicial.

Verica-se, então, que um índice repetido faz com que a expressão cresça na direção horizontal ao se aplicar à convenção do somatório. Por sua vez, o índice livre indica o número total de equações, fazendo com que a expressão em notação indicial se expanda na direção vertical. Esta idéia esta ilustrada na Figura A.1. 


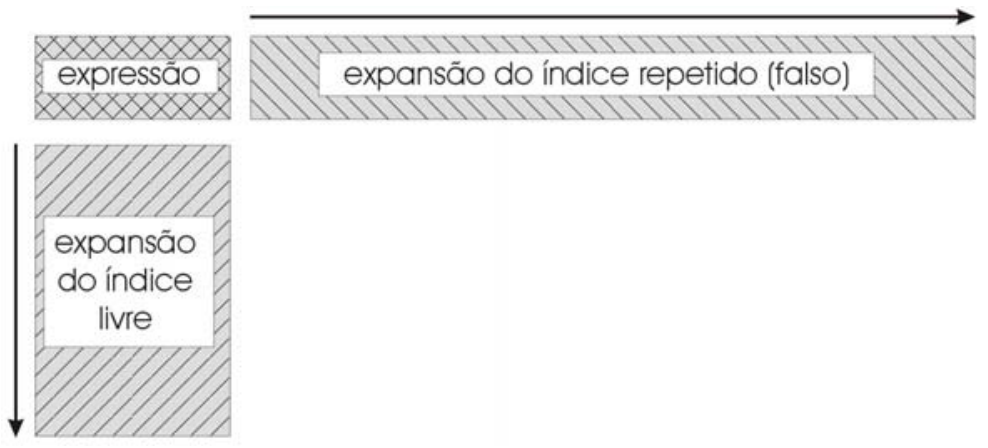

Figura A.1: Índices livre e repetido

\section{a.3) Permutação}

A Figura A.2 ilustra os índices i, j, k e 1, 2, 3 ordenados nos sentidos horário e antihorário. Utilizam-se estes índices para de unir o símbolo de permutação $\mathrm{e}_{\mathrm{ijk}}$ da seguinte forma

$$
\left\{\begin{array}{cc}
\text { e123 }=\mathrm{e} 231=\mathrm{e} 312 & 1,2,3 \text { no sentido horario } \\
\mathrm{e} 213=\mathrm{e} 132=\mathrm{e} 321 & 1,2,3 \text { no sentido anti-horario } \\
\text { eijk }=0 & \text { nos demais casos }
\end{array}\right.
$$

Em outras palavras, o termo $\mathrm{e}_{\mathrm{ijk}}$ se anula sempre que os valores de quaisquer dois índices coincidem, como por exemplo $\mathrm{e}_{112}=0$. Por sua vez, $\mathrm{e}_{\mathrm{ijk}}=1$ quando os subscritos permutam na ordem $1,2,3$, ou seja, no sentido horário. Finalmente, $\mathrm{e}_{\mathrm{ijk}}=-1$ caso a permutação seja no sentido horário.

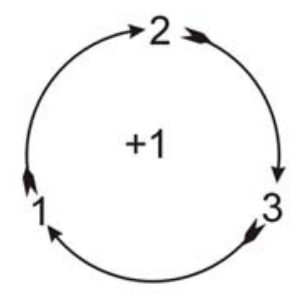

(a) 123 em sentido horário.

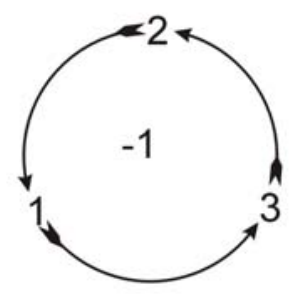

(b) 123 em sentido anti-horário.

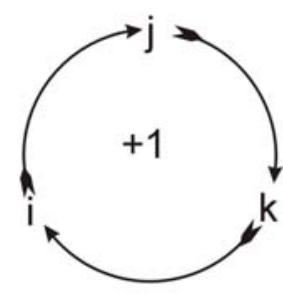

(c) ijk em sentido horário.

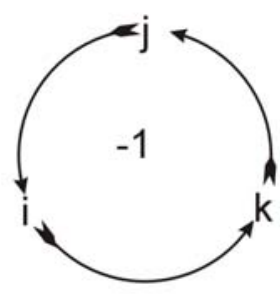

(d) ijk em sentido anti-horário.

Figura A.2: Símbolo de permutação.

Como exemplo de aplicação, considere o determinante $|\mathrm{A}|$ de uma matriz [A] 
$|A|=\left|\begin{array}{ccc}a_{11} & a_{12} & a_{13} \\ a_{21} & a_{22} & a_{23} \\ a_{31} & a_{32} & a_{33}\end{array}\right|=a_{11} a_{22} a_{33}+a_{21} a_{32} a_{13}+a_{31} a_{12} a_{23}-a_{11} a_{31} a_{23}-a_{21} a_{12} a_{33}-a_{31} a_{22} a_{13}$

A equação anterior pode ser denotada como

$|\mathrm{A}|=\mathrm{e}_{\mathrm{ijk}} \mathrm{a}_{\mathrm{i} 1} \mathrm{a}_{\mathrm{j} 2} \mathrm{a}_{\mathrm{k} 3}=\sum_{\mathrm{i}, \mathrm{j}, \mathrm{k}=1}^{3} \mathrm{e}_{\mathrm{ijk}} \mathrm{a}_{\mathrm{i} 1} \mathrm{a}_{\mathrm{j} 2} \mathrm{a}_{\mathrm{k} 3}=\sum_{\mathrm{i}=1}^{3} \sum_{\mathrm{j}=1}^{3} \sum_{\mathrm{k}=1}^{3} \mathrm{e}_{\mathrm{ijk}} \mathrm{a}_{\mathrm{i} 1} \mathrm{a}_{\mathrm{j} 2} \mathrm{a}_{\mathrm{k} 3}$

sendo $\mathrm{i}, \mathrm{j}$, k índices livres e $\mathrm{e}_{\mathrm{ijk}} \mathrm{o}$ símbolo de permutação.

O delta de Kronecker e o símbolo de permutação estão associados pela identidade podendo assim ser escrito:

$\mathrm{e}_{\mathrm{ijk}} \mathrm{e}_{\mathrm{klm}}=\delta_{\mathrm{ik}} \delta_{\mathrm{jl}}-\delta_{\mathrm{il}} \delta_{\mathrm{jk}}$

como pode ser comprovado manipulando-se os índices.

\section{a.4) Substituição}

Considere as seguintes relações

$\mathrm{a}_{\mathrm{i}}=\mathrm{U}_{\mathrm{im}} \mathrm{b}_{\mathrm{m}}$

$\mathrm{b}_{\mathrm{i}}=\mathrm{V}_{\mathrm{mn}} \mathrm{c}_{\mathrm{n}}$

Observa-se que o termo $\mathrm{b}$ aparece nas duas relações, mas com índices distintos. Deseja-se substituir b dado em (A.8) na expressão (A.7). Para isso, muda-se o índice livre de i para m em (A.8), obtendo-se

$\mathrm{b}_{\mathrm{m}}=\mathrm{V}_{\mathrm{mm}} \mathrm{c}_{\mathrm{m}}$

No entanto, a expressão resultante não é válida em notação indicial, pois o índice $\mathrm{m}$ está repetido mais de uma vez no lado direito da equação. Para resolver este problema, lembre-se que a letra empregada para um índice falso num termo não afeta o resultado, ou 
seja, $V_{\text {im }} c_{m}=V_{\text {in }} c_{n}$. Logo, alterando o índice falso de m para $n$ em (A.8) e o índice livre de i para $\mathrm{m}$ vem que

$\mathrm{b}_{\mathrm{m}}=\mathrm{V}_{\mathrm{mn}} \mathrm{c}_{\mathrm{n}}$

Como agora tem-se o mesmo índice m nas expressões (A.8) e (A.9), efetua-se a substituição

$\mathrm{a}_{\mathrm{i}}=\mathrm{U}_{\mathrm{im}} \mathrm{b}_{\mathrm{m}}=\mathrm{U}_{\mathrm{im}} \mathrm{V}_{\mathrm{mn}} \mathrm{c}_{\mathrm{n}}$

Observe que (A.10) representa três equações ao se variar o índice livre i de 1 a 3. Por sua vez, cada equação resulta numa soma de nove termos no lado direito, pois os índices repetidos m e n variam cada um de 1 a 3 . Logo

$$
a_{i}=U_{i m} V_{m n} c_{n} \rightarrow\left\{\begin{array}{l}
a_{1}=\sum_{m, n=1}^{3} U_{1 m} V_{m n} c_{n}=\sum_{m=1}^{3} \sum_{n=1}^{3} U_{1 m} V_{m n} c_{n} \\
a_{2}=\sum_{m, n=1}^{3} U_{2 m} V_{m n} c_{n}=\sum_{m=1}^{3} \sum_{n=1}^{3} U_{2 m} V_{m n} c_{n} \\
a_{3}=\sum_{m, n=1}^{3} U_{3 m} V_{m n} c_{n}=\sum_{m=1}^{3} \sum_{n=1}^{3} U_{3 m} V_{m n} c_{n}
\end{array}\right.
$$

De forma geral, deve-se ter cuidado ao se fazer substituições convenientes, ou seja, não substituir índices repetidos por livres, podendo dar origem a um somatório inexistente na notação indicial.

\section{a.5) Multiplicação}

Considere a multiplicação de $\mathrm{p}$ e q dados respectivamente por

$$
\begin{aligned}
& \mathrm{p}=\mathrm{a}_{\mathrm{m}} \mathrm{b}_{\mathrm{m}}=\sum_{\mathrm{m}=1}^{3} \mathrm{a}_{\mathrm{m}} \mathrm{b}_{\mathrm{m}}=\mathrm{a}_{1} \mathrm{~b}_{1}+\mathrm{a}_{2} \mathrm{~b}_{2}+\mathrm{a}_{3} \mathrm{~b}_{3}, \\
& \mathrm{q}=\mathrm{c}_{\mathrm{m}} \mathrm{d}_{\mathrm{m}}=\sum_{\mathrm{m}=1}^{3} \mathrm{c}_{\mathrm{m}} \mathrm{d}_{\mathrm{m}}=\mathrm{c}_{1} \mathrm{~d}_{1}+\mathrm{c}_{2} \mathrm{~d}_{2}+\mathrm{c}_{3} \mathrm{~d}_{3} .
\end{aligned}
$$

A partir daí, o produto pq é calculado como 
$p q=\left(a_{1} b_{1}+a_{2} b_{2}+a_{3} b_{3}\right)\left(c_{1} d_{1}+c_{2} d_{2}+c_{3} d_{3}\right)$

Podendo ainda ser denotado em notação indicial

$p q=\left(\sum_{m=1}^{3} a_{m} b_{m}\right)\left(\sum_{n=1}^{3} c_{n} d_{n}\right)=\sum_{m, n=1}^{3} a_{m} b_{m} c_{n} d_{n}=a_{m} b_{m} c_{n} d_{n}$

Portanto, o produto pq é indicado em notação indicial como $p q=a_{m} b_{m} c_{n} d_{n}$. É importante notar que para obter o produto pq não basta simplesmente multiplicar p e q dados em (A.12), ou seja, $p q \neq a_{m} b_{m} c_{m} d_{m}$ pois

$a_{m} b_{m} c_{m} d_{m}=\sum_{m=1}^{3} a_{m} b_{m} c_{m} d_{m}=a_{1} b_{1} c_{1} d_{1}+a_{2} b_{2} c_{2} d_{2}+a_{3} b_{3} c_{3} d_{3}$

De fato, o termo $a_{m} b_{m} c_{m} d_{m}$ não possui significado na convenção de somatório, pois o índice repetido m aparece mais de uma vez num mesmo termo. Logo, ao se efetuar o produto de termos em notação indicial, deve-se inicialmente compatibilizar os índices. No caso anterior, trocou-se o índice repetido $m$ para $n$ no termo $q=c_{m} d_{m}=c_{n} d_{n}$. Lembre-se que a letra usada para o índice repetido é irrelevante, ou seja, para o exemplo considerado $\mathrm{c}_{\mathrm{m}} \mathrm{d}_{\mathrm{m}}=\mathrm{c}_{\mathrm{n}} \mathrm{d}_{\mathrm{n}}=\mathrm{c}_{\mathrm{j}} \mathrm{d}_{\mathrm{j}}=\mathrm{c}_{\mathrm{k}} \mathrm{d}_{\mathrm{k}}=\ldots$.

\section{a.6) Fatoração}

Considere a seguinte expressão

$\mathrm{T}_{\mathrm{ij}} \mathrm{n}_{\mathrm{j}}-\lambda \mathrm{n}_{\mathrm{i}}=0$,

a qual define um problema de autovalor do tensor $\mathrm{T}_{\mathrm{ij}}$, como ser. Verifica-se que na expressão anterior i e j são, respectivamente, índices livres e repetidos. Em particular, empregam-se estes dois índices para o termo $\mathrm{n}$. Para uniformizar os índices em $\mathrm{n}$ e fatorar a 
expressão, colocando o termo $n_{j}$ em evidência, emprega-se o delta de Kronecker de tal forma que $\mathrm{n}_{\mathrm{i}}=\delta_{\mathrm{ij}} \mathrm{n}_{\mathrm{j}}$. Logo, verifica-se que

$\mathrm{T}_{\mathrm{ij}} \mathrm{n}_{\mathrm{j}}-\lambda \delta_{\mathrm{ij}} \mathrm{n}_{\mathrm{j}}=0 \rightarrow\left(\mathrm{T}_{\mathrm{ij}}-\lambda \delta_{\mathrm{ij}}\right) \mathrm{n}_{\mathrm{j}}=0$.

Observa-se que a expressão anterior pode ser denotada matricialmente como

$([\mathrm{T}]-\lambda[\mathrm{I}])\{\mathrm{n}\}=\{0\}$,

ou seja, tem-se a forma padrão de um problema de autovalor. De forma geral, para se fatorar um termo denotado em notação indicial, deve-se compatibilizar os índices empregando o delta de Kronecker ou o símbolo de permutação.

\section{a.7) Contração}

A operação de igualar dois índices distintos e somar os mesmos é conhecida como contração. Por exemplo, $T_{\text {ii }}$ é a contração de Tij, ou seja,

$$
\mathrm{T}_{\mathrm{ii}}=\mathrm{T}_{11}+\mathrm{T}_{22}+\mathrm{T}_{33}
$$

\section{a.8) Diferenciação}

As operações de derivação (gradiente, divergente e etc) também podem ser representadas via notação indicial. Observe os seguintes exemplos, respectivamente, para as derivadas total e parcial de $u$

$$
\begin{gathered}
\frac{\mathrm{du}}{\mathrm{dx}_{\mathrm{i}}}=\mathrm{u}_{, \mathrm{i}}, \\
\frac{\partial \mathrm{u}}{\partial \mathrm{x}_{\mathrm{i}}}=\mathrm{u}_{, \mathrm{i}} .
\end{gathered}
$$


Considerando uma função $\mathrm{u}=\mathrm{u}\left(\mathrm{a}_{\mathrm{j}}\left(\mathrm{x}_{\mathrm{i}}\right)\right)$, emprega-se a regra da cadeia para obter a derivada $\frac{\partial \mathrm{u}}{\partial \mathrm{x}_{\mathrm{i}}}$ da função $\mathrm{u}$ com relação a $\mathrm{x}_{\mathrm{i}}$, ou seja,

$$
\frac{\partial \mathrm{u}}{\partial \mathrm{x}_{\mathrm{i}}}=\mathrm{u}_{, \mathrm{i}}=\frac{\partial \mathrm{u}}{\partial \mathrm{a}_{\mathrm{j}}} \frac{\partial \mathrm{a}_{\mathrm{j}}}{\partial \mathrm{x}_{\mathrm{i}}}=\mathrm{u}_{, \mathrm{j}} \mathrm{u}_{\mathrm{j}, \mathrm{i}}
$$

Considerando uma função escalar $\mathrm{a}=\mathrm{a}\left(\mathrm{x}_{\mathrm{i}}\right)$, o seu gradiente em notação indicial é denotado como

$$
\nabla \mathrm{a}=\frac{\partial \mathrm{a}}{\partial \mathrm{x}_{1}} \mathrm{e}_{1}+\frac{\partial \mathrm{a}}{\partial \mathrm{x}_{2}} \mathrm{e}_{2}+\frac{\partial \mathrm{a}}{\partial \mathrm{x}_{3}} \mathrm{e}_{3}=\mathrm{a}_{, \mathrm{i}} \mathrm{e}_{\mathrm{i}}
$$

Por sua vez, o divergente de uma função vetorial $\mathrm{u}=\mathrm{u}\left(\mathrm{x}_{\mathrm{i}}\right)$ é expresso como

$$
\operatorname{divu}=\nabla \cdot \mathrm{u}=\frac{\partial \mathrm{u}_{1}}{\partial \mathrm{x}_{1}}+\frac{\partial \mathrm{u}_{2}}{\partial \mathrm{x}_{2}}+\frac{\partial \mathrm{u}_{3}}{\partial \mathrm{x}_{3}}=\mathrm{u}_{\mathrm{i}, \mathrm{i}}
$$

Já o rotacional de um é dado por

$$
\nabla \times \mathrm{u}=\mathrm{e}_{\mathrm{ijk}} \frac{\partial \mathrm{u}_{\mathrm{k}}}{\partial \mathrm{x}_{\mathrm{j}}} \mathrm{e}_{\mathrm{i}}=\mathrm{e}_{\mathrm{ijk}} \mathrm{u}_{\mathrm{k}, \mathrm{j}} \mathrm{e}_{\mathrm{i}}
$$


Problema de VAlor de Contorno

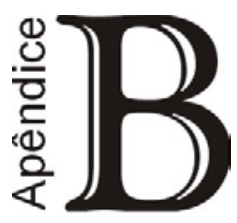

Um problema de valor de contorno envolve a aplicação de uma equação diferencial a um domínio $\Omega$, limitado por um contorno $\Gamma$ (Fig B.1). Como exemplo pode-se citar a equação de Poisson

$\nabla^{2} \phi(\mathrm{x}, \mathrm{y})=\mathrm{b}(\mathrm{x}, \mathrm{y})$ em $\Omega$,

Onde $\phi(\mathrm{x}, \mathrm{y})$ é a função incógnita (neste caso, um potencial) a ser determinada e $\mathrm{b}(\mathrm{x}, \mathrm{y})$ é uma função definida no domínio $\Omega$.

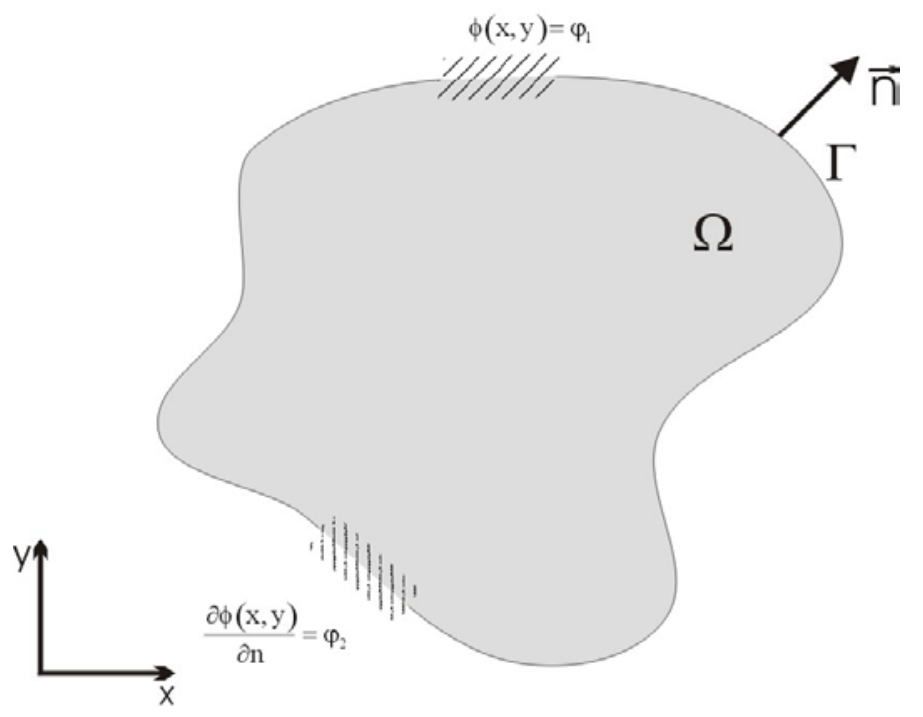

Figura B.1: Problema de valor de contorno. 
A obtenção da solução de uma equação diferencial exige a especificação das condições de contorno, estabelecendo o valor da função incógnita, ou de suas derivadas, em trechos do contorno $\Gamma$. Essas condições de contorno podem ser de dois tipos:

- Valores prescritos da função incógnita $\phi$ :

$\phi(\mathrm{x}, \mathrm{y})=\varphi_{1}$,

Também conhecidas como condições essenciais ou condições de Dirichlet.

Valores prescritos da derivada da função incógnita em relação à normal ao contorno:

$\frac{\partial \phi(\mathrm{x}, \mathrm{y})}{\partial \mathrm{n}}=\varphi_{2}$

também conhecidas como condições naturais ou condições de Neumann.

O contorno pode ser definido como o lugar geométrico dos pontos em cuja vizinhança existem tantos pontos internos quanto pontos externos em relação ao domínio. Em relação ao domínio pode ser de dois tipos: externo ou interno. Dependendo do tipo de contorno, o Domínio pode ser classificado como fechado ou aberto e o sentido de numeração dos nós do contorno será, respectivamente, anti-horário ou horário. Estas definições de domínio aberto ou fechado e de contorno externo ou interno podem claramente ser vistas na figura abaixo:
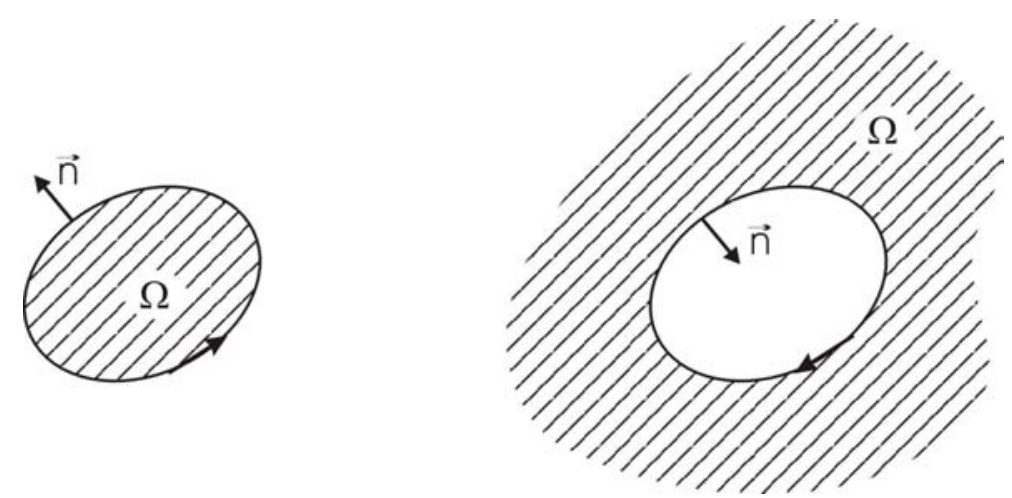

(A) Domínio Fechado

(B) Domínio Fechado

Figura B.2: Classificação dos domínios segundo: BREBBIA \& DOMINGUES (1989). 
Contudo é importante salientar que este tipo de classificação não passa de mera formalidade acadêmica com um intuito apenas didático de explicar a diferença que ha no sentido de orientação dos elementos de contorno. Pois modelos um pouco mais elaborados com sub-regiões, buracos e etc. não se encaixam muito bem a este tipo de classificação sugerido anteriormente, como pode ser observado no exemplo abaixo.

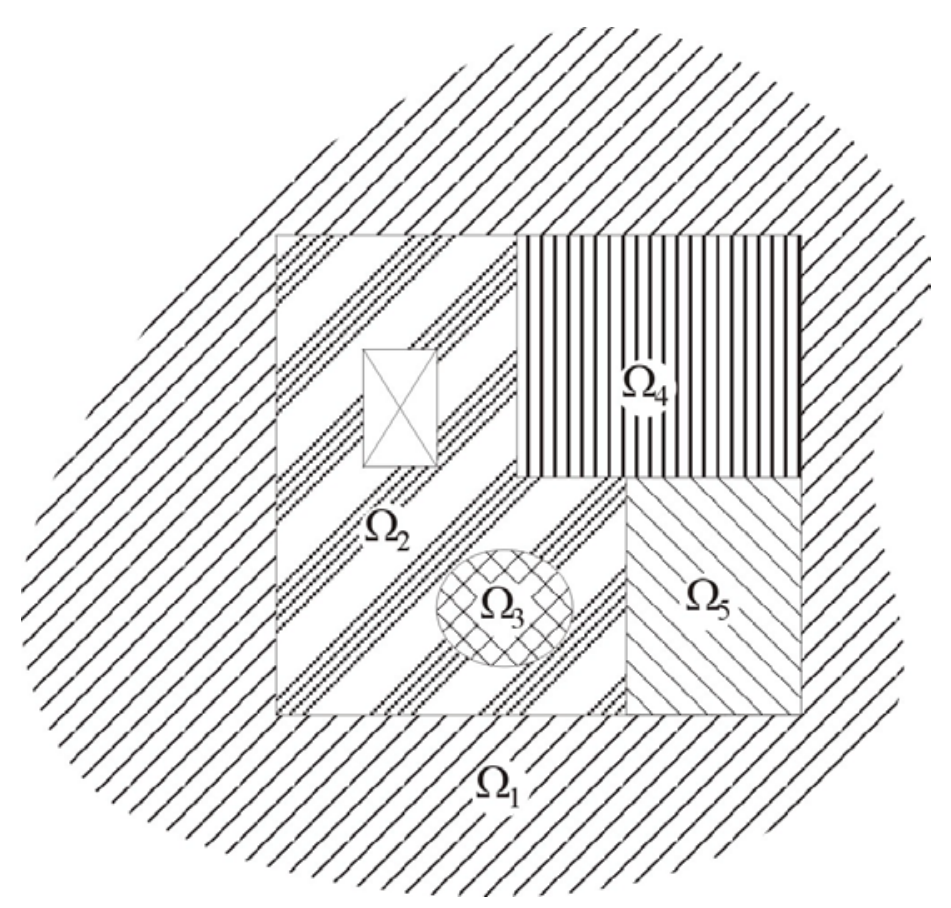

Figura B.3: Situação Intermediária( domínios aberto e fechados). 